\title{
Ni/AntPohs-Catalyzed Stereoselective Asymmetric Intramolecular Reductive Coupling of $\mathrm{N}$-1,6-Alkynones
}

Wanjun Chen, ${ }^{\ddagger a}$ Yaping Cheng, ${ }^{\ddagger \mathrm{a}}$ Tao Zhang, ${ }^{\mathrm{a}} \mathrm{Yu} \mathrm{Mu},{ }^{\mathrm{a}}$ Wenqi Jia ${ }^{\mathrm{a}}$ and Guodu Liu*ab

\author{
a. Key Laboratory of Fine Organic Synthesis of Inner Mongolia, College of Chemistry and Chemical Engineering, Inner \\ Mongolia University, 235 Daxue West Rd, Hohhot 010021, China. \\ Email: guoduliu@imu.edu.cn \\ b. State Key Laboratory of Bio-organic and Natural Products Chemistry, Shanghai Institute of Organic Chemistry, Chinese \\ Academy of Sciences, 345 Ling Ling Rd, Shanghai 200032, China.
}

\section{Table of Contents}

1. General Information and Materials.

2. Substrates Synthesis. 


\section{General Information and Materials}

All reactions and manipulations were performed in a nitrogen-filled glove box or using standard Schlenk techniques, unless otherwise noted. All anhydrous solvents were purchased from J \& K Chemicals or Alfa Chemicals Inc, or used after standard purification procedures. Ni(cod) $)_{2}$ was purchased from Strem chemicals. Commercialized reagents were used without further purifications. All air sensitive ligands were stored in a nitrogen-filled glove box before use. Chiral ligand intermediates were prepared according to our reported procedures.

${ }^{1} \mathrm{H}$, and ${ }^{13} \mathrm{C}$ NMR data were recorded on a BrukerDRX500, DRX400, NMR Spectrometer with $\mathrm{CDCl}_{3}$, or $\mathrm{CD}_{3} \mathrm{OD}$ as the solvent. ${ }^{1} \mathrm{H}$ chemical shifts were referenced to $\mathrm{CDCl}_{3}$ at $7.26 \mathrm{ppm} .{ }^{13} \mathrm{C}$ chemical shifts were referenced to $\mathrm{CDCl}_{3}$ at $77.16 \mathrm{ppm}$ and obtained with ${ }^{1} \mathrm{H}$ decoupling. HRMS was measured on Waters Xevo G2-XS QTof( ZSpray\&trade; Biorthogonal API source ) mass spectrometers. HPLC analyses and purifications were performed on a Thermo Fisher LC system with UV/VIS detector using chiralcel columns.

\section{Substrates Synthesis}
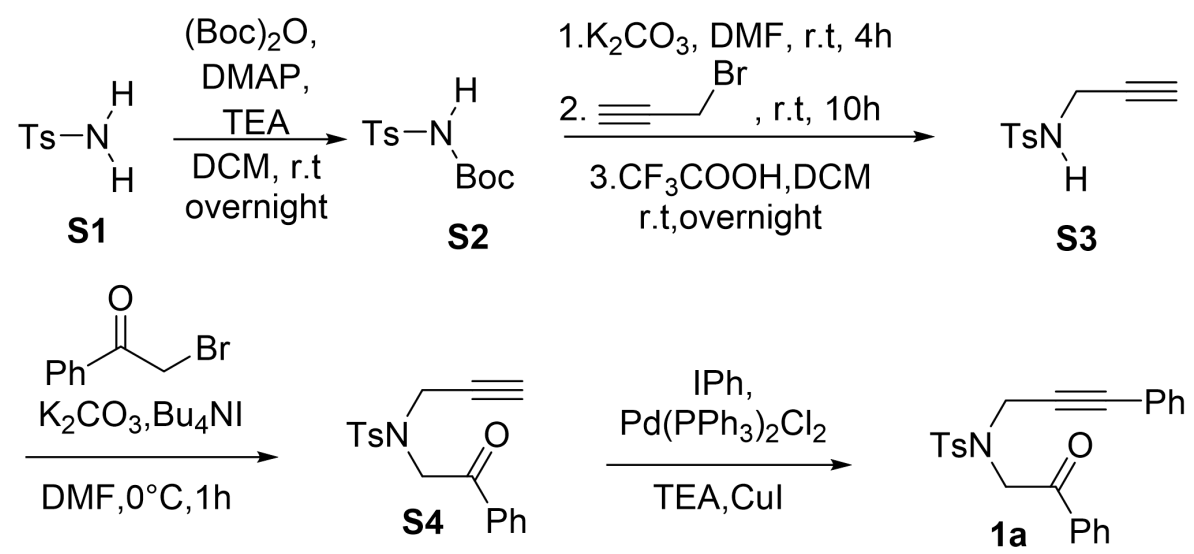

Scheme S1. General procedure for substrate 1a. 


\section{HPLC Spectra of Chiral Products}

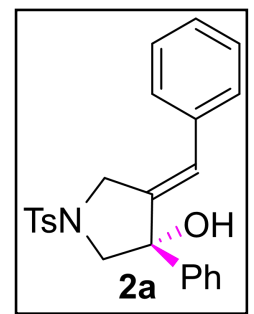

2a: white solid, $(80.3 \mathrm{mg}, 99 \%), 99: 1$ er. $[\alpha]_{\mathrm{D}^{25}}=-22.0\left(\mathrm{c}=1.0, \mathrm{CH}_{2} \mathrm{Cl}_{2}\right)$; Enantiomeric ratio was determined by chiral HPLC. Chiralpark AD-H, $25{ }^{\circ} \mathrm{C}$, flow rate: $1 \mathrm{~mL} / \mathrm{min}$, hexanes/isopropanol: 15/85, $254 \mathrm{~nm}, 7.08 \mathrm{~min}(S), 8.11$ $\min (R)$.
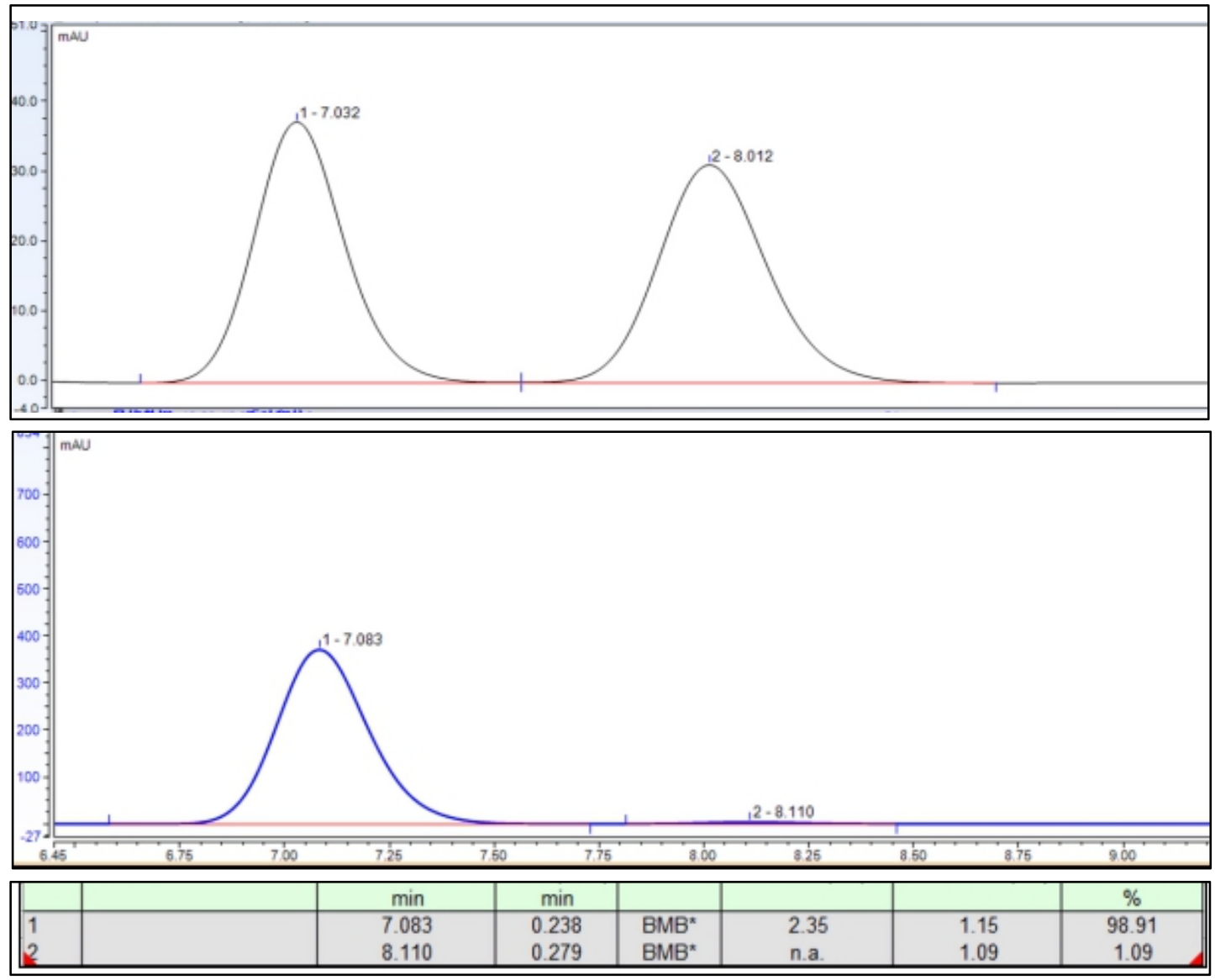

Figure Sl. HPLC chromatogram of chiral product 2a. 

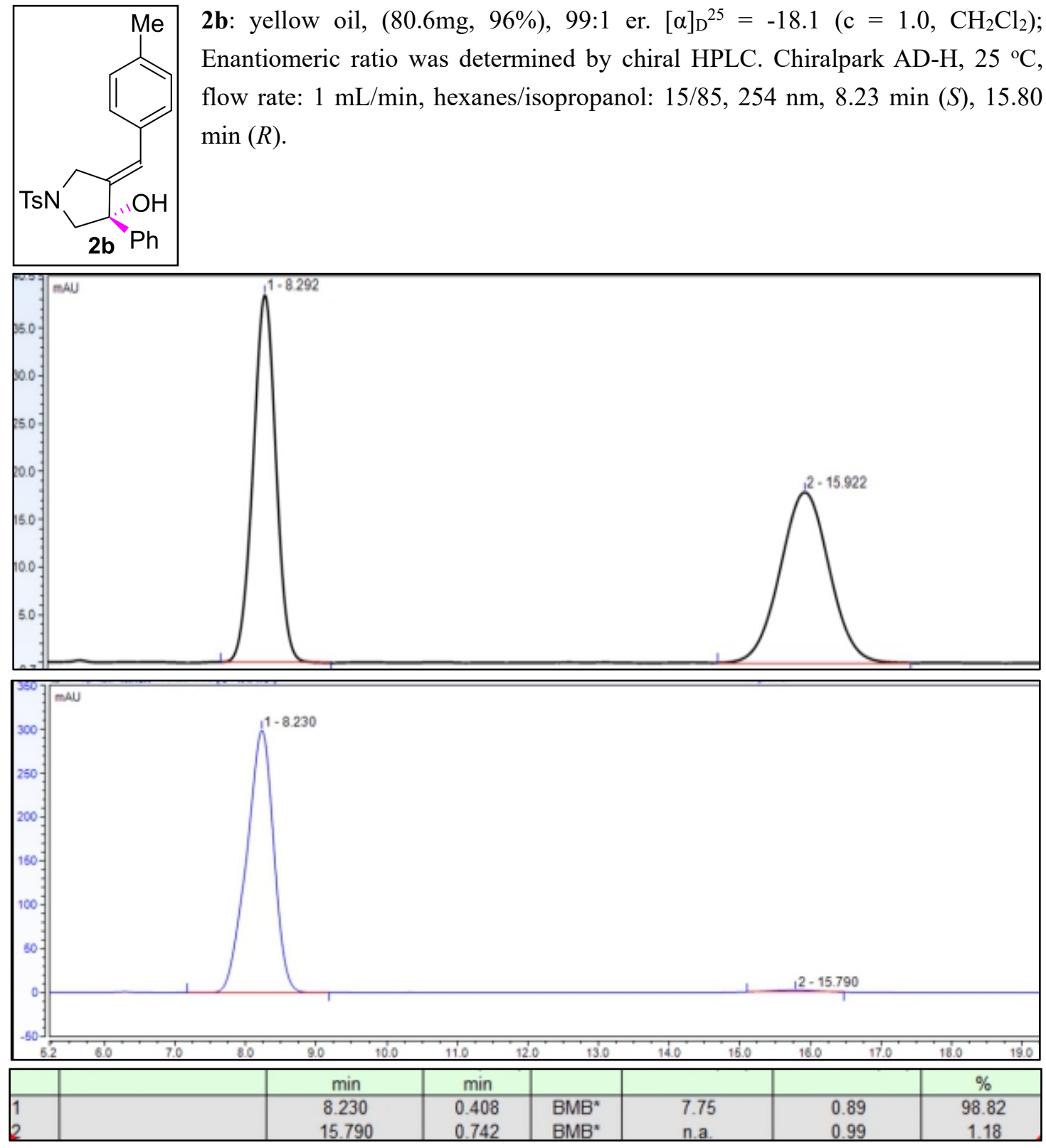

Figure S2. HPLC chromatogram of chiral product $\mathbf{2 b}$. 


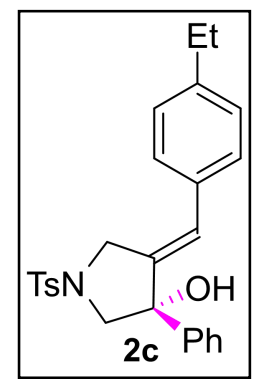

2c: white solid, $(78.0 \mathrm{mg}, 90 \%), 99: 1$ er. $[\alpha]_{\mathrm{D}}{ }^{25}=-29.3\left(\mathrm{c}=1.0, \mathrm{CH}_{2} \mathrm{Cl}_{2}\right)$; Enantiomeric ratio was determined by chiral HPLC. Chiralpark AD-H, $25{ }^{\circ} \mathrm{C}$, flow rate: $0.5 \mathrm{~mL} / \mathrm{min}$, hexanes/isopropanol: 20/80, $254 \mathrm{~nm}, 6.58 \mathrm{~min}(R), 13.86$ $\min (S)$.

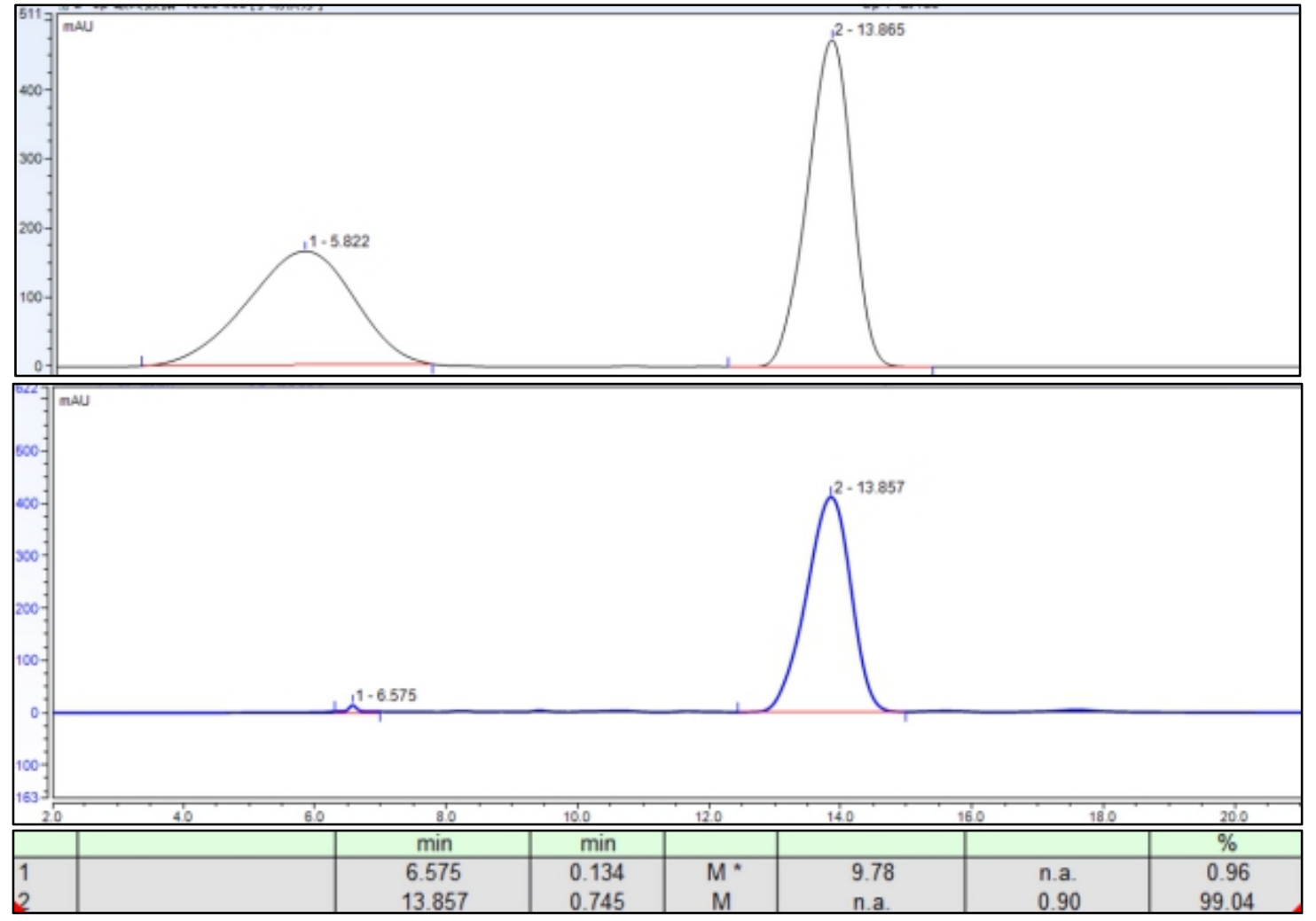

Figure S3. HPLC chromatogram of chiral product 2c. 

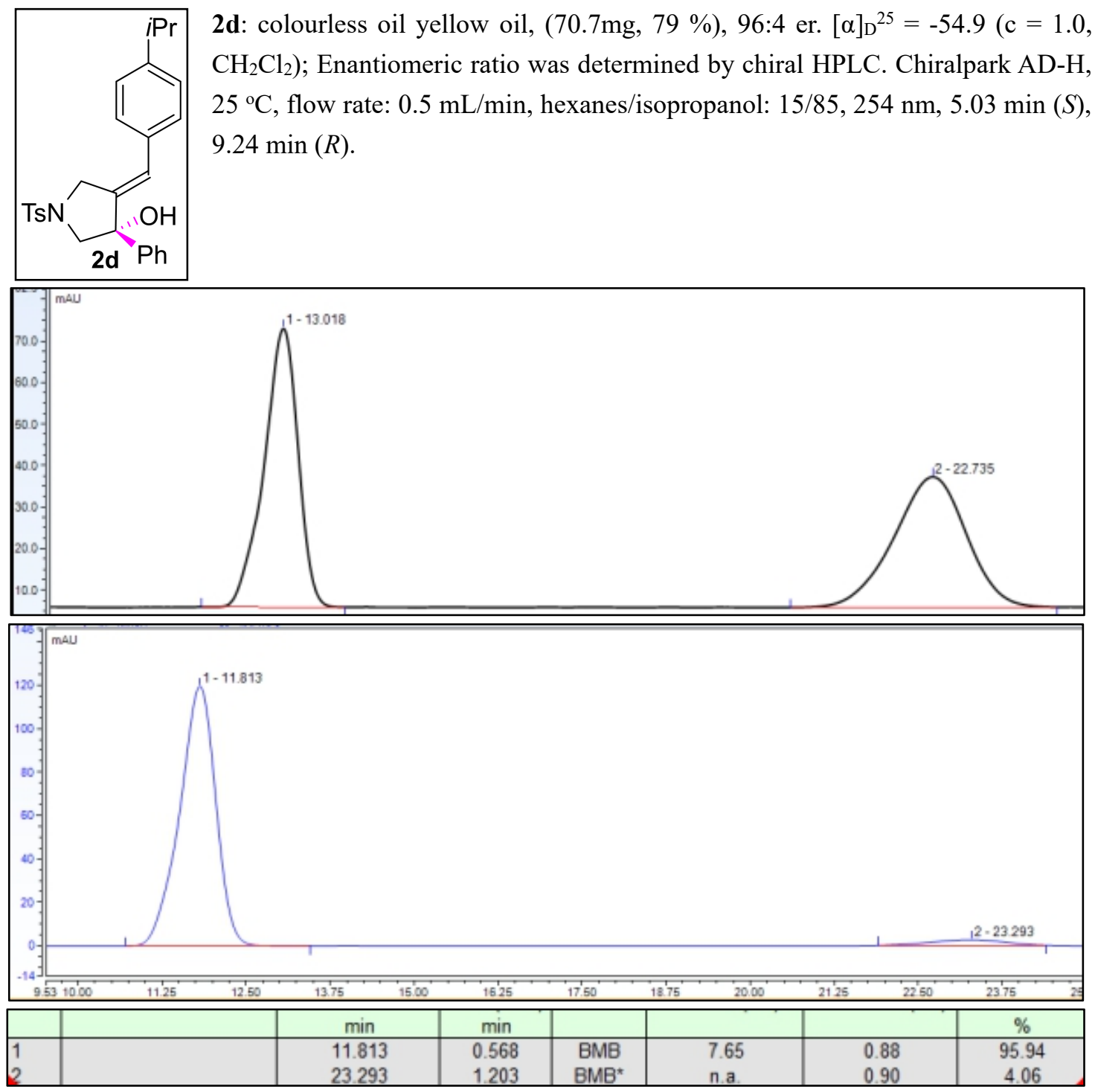

Figure S4. HPLC chromatogram of chiral product 2d. 


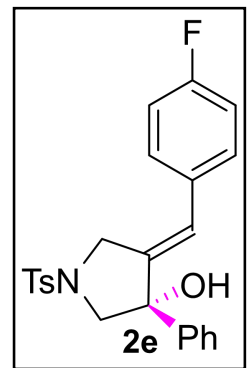

2e: white solid, $(70.3 \mathrm{mg}, 83 \%), 95: 5$ er. $[\alpha]_{\mathrm{D}}{ }^{25}=-36.6\left(\mathrm{c}=1.0, \mathrm{CH}_{2} \mathrm{Cl}_{2}\right)$; Enantiomeric ratio was determined by chiral HPLC. Chiralpark AD-H, $25{ }^{\circ} \mathrm{C}$, flow rate: $1 \mathrm{~mL} / \mathrm{min}$, hexanes/isopropanol: 20/80, $254 \mathrm{~nm}, 10.41 \mathrm{~min}(S), 13.87$ $\min (R)$.
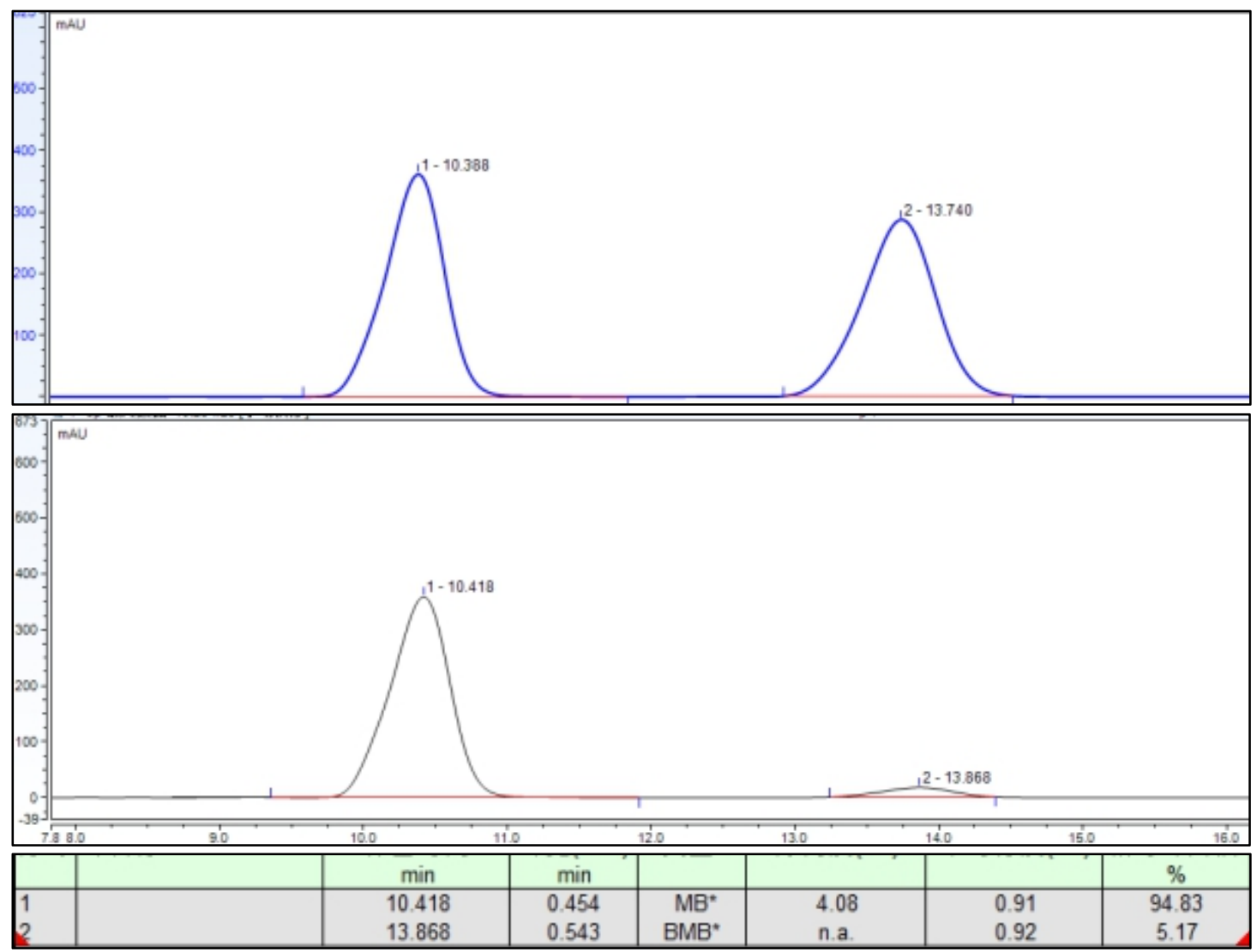

Figure S5. HPLC chromatogram of chiral product $2 \mathbf{e}$. 


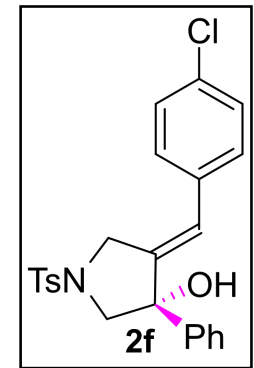

2f: colorless oil, $(77.3 \mathrm{mg}, 88 \%), 99: 1$ er, $[\alpha]_{\mathrm{D}}{ }^{25}=-32.25$ (c $=1.0$, $\mathrm{CH}_{2} \mathrm{Cl}_{2}$ ); Enantiomeric ratio was determined by chiral HPLC. Chiralpark AD-H, $25{ }^{\circ} \mathrm{C}$, flow rate: $0.5 \mathrm{~mL} / \mathrm{min}$, hexanes/isopropanol: 15/85, $254 \mathrm{~nm}, 6.90 \mathrm{~min}$ $(S), 12.03 \min (R)$.
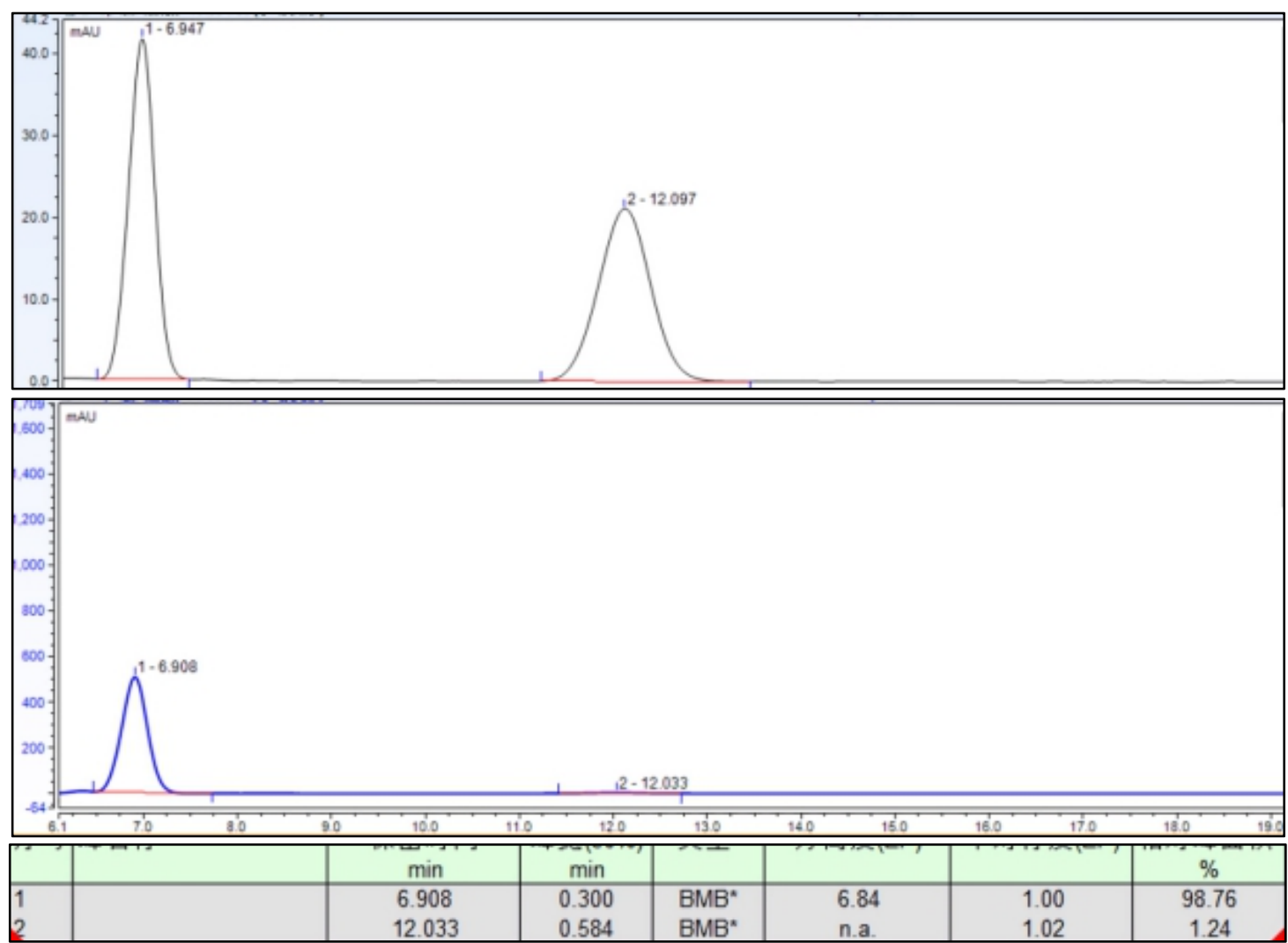

Figure S6. HPLC chromatogram of chiral product $\mathbf{2 f}$. 


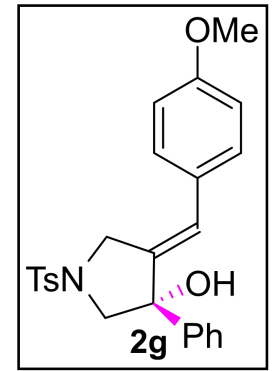

2g: white solid, (83.6mg, 96\%), 99:1 er. $[\alpha]_{\mathrm{D}}^{25}=-36.1\left(\mathrm{c}=1.0, \mathrm{CH}_{2} \mathrm{Cl}_{2}\right)$; Enantiomeric ratio was determined by chiral HPLC. Chiralpark AD-H, $25{ }^{\circ} \mathrm{C}$, flow rate: $0.5 \mathrm{~mL} / \mathrm{min}$, hexanes/isopropanol: 20/80, $254 \mathrm{~nm}, 7.93 \mathrm{~min}(S)$, $20.91 \min (R)$.

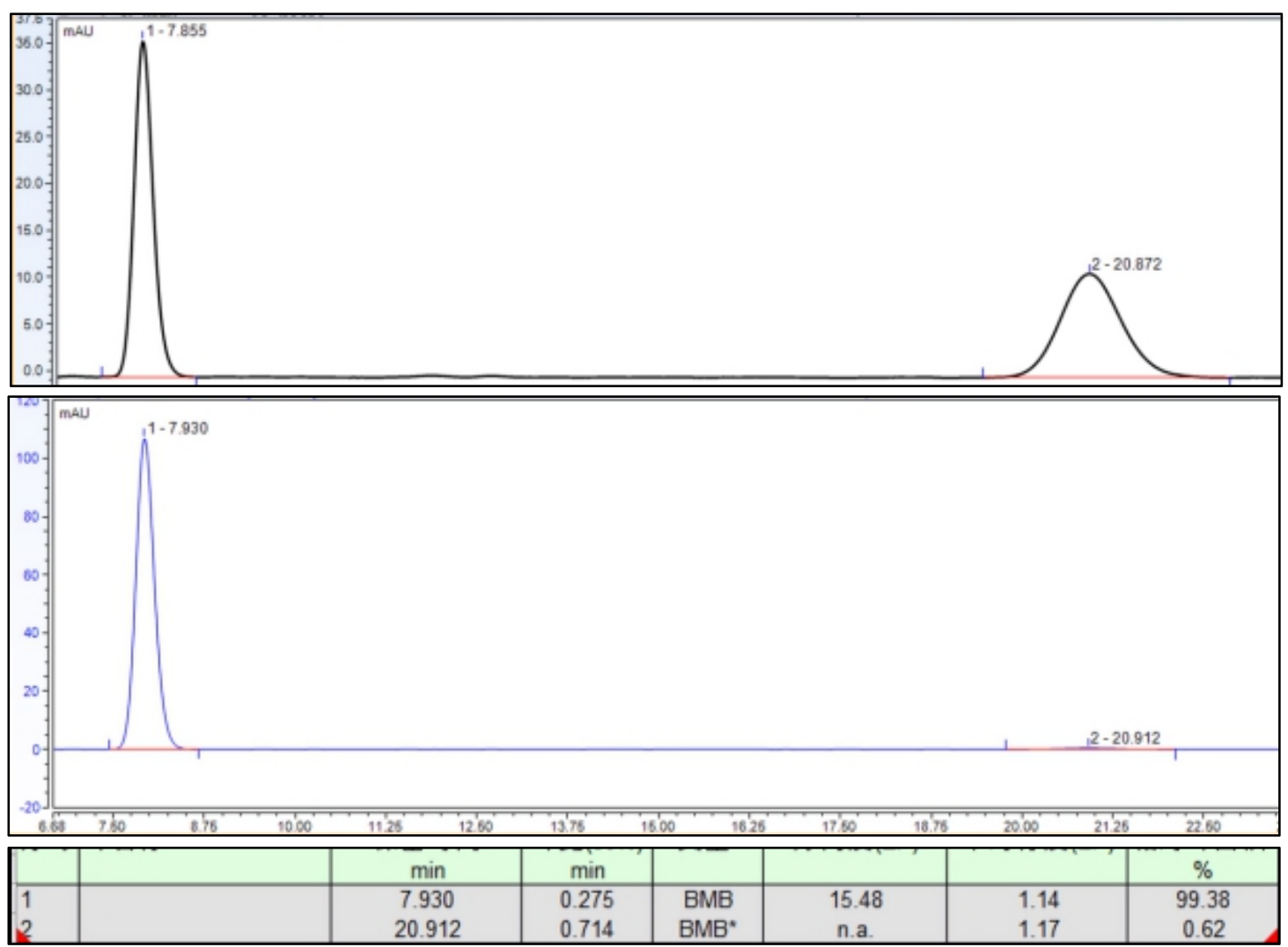

Figure S7. HPLC chromatogram of chiral product 2g. 

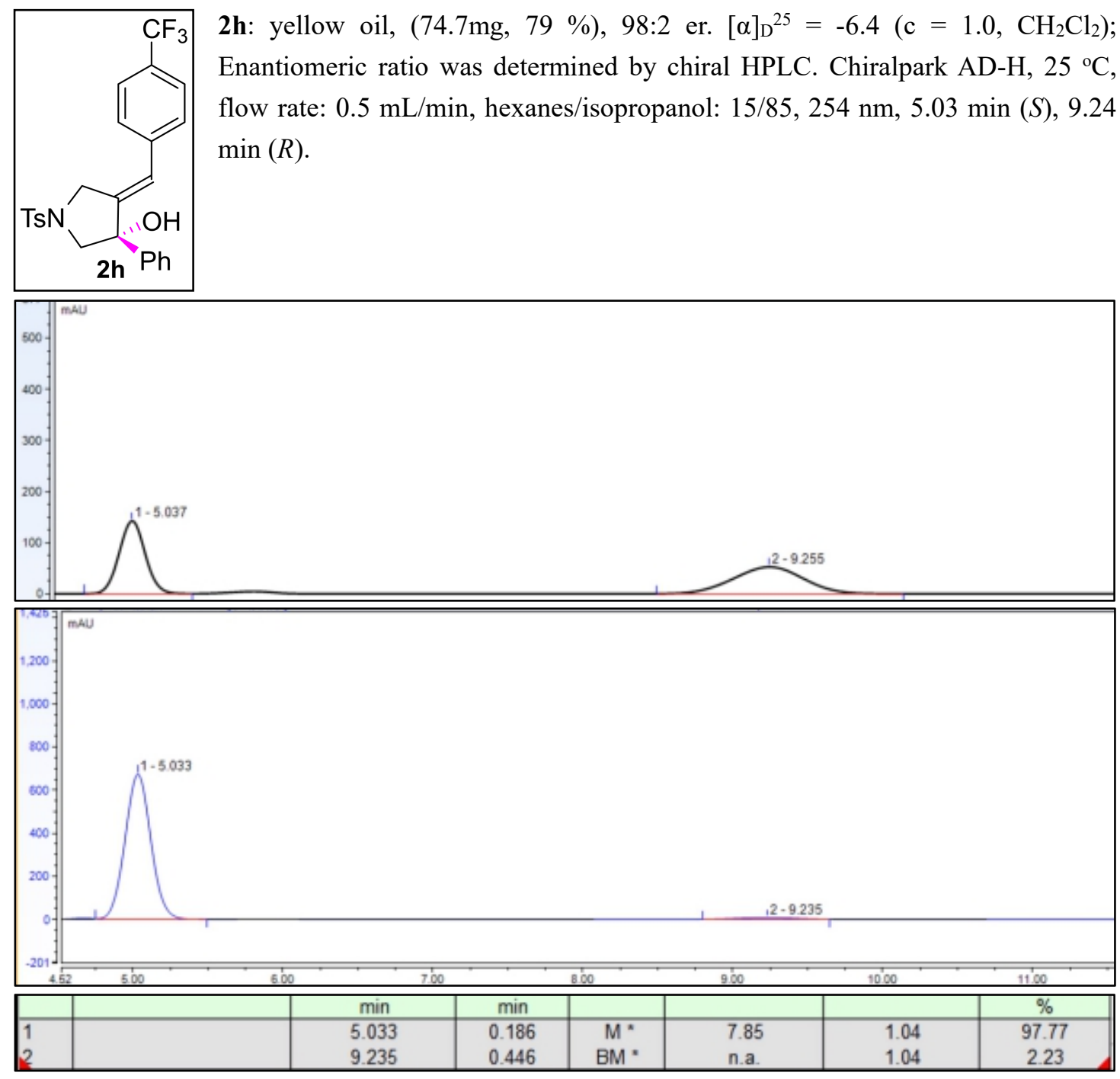

Figure S8. HPLC chromatogram of chiral product $\mathbf{2 h}$. 


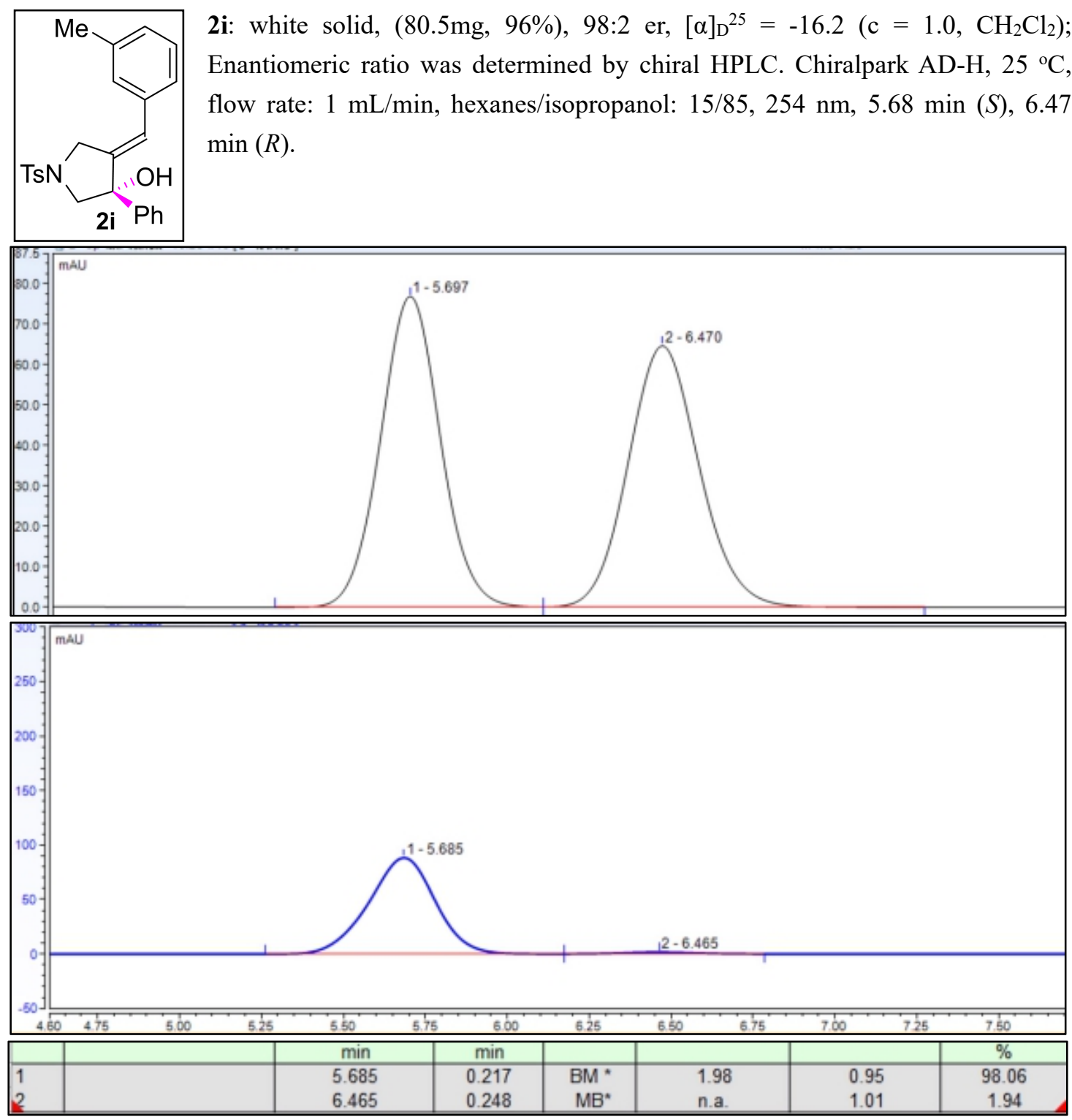

Figure S9. HPLC chromatogram of chiral product $\mathbf{2 i}$. 


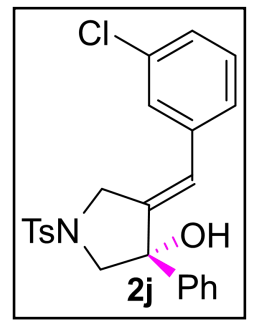

2j: white solid, $(75.7 \mathrm{mg}, 86 \%), 98: 2$ er. $[\alpha]_{\mathrm{D}}^{25}=-16.5\left(\mathrm{c}=1.0, \mathrm{CH}_{2} \mathrm{Cl}_{2}\right)$; Enantiomeric ratio was determined by chiral HPLC. Chiralpark AD-H, $25{ }^{\circ} \mathrm{C}$, flow rate: $1 \mathrm{~mL} / \mathrm{min}$, hexanes/isopropanol: 15/85, $254 \mathrm{~nm}, 5.39 \mathrm{~min}(S), 6.25$ $\min (R)$.
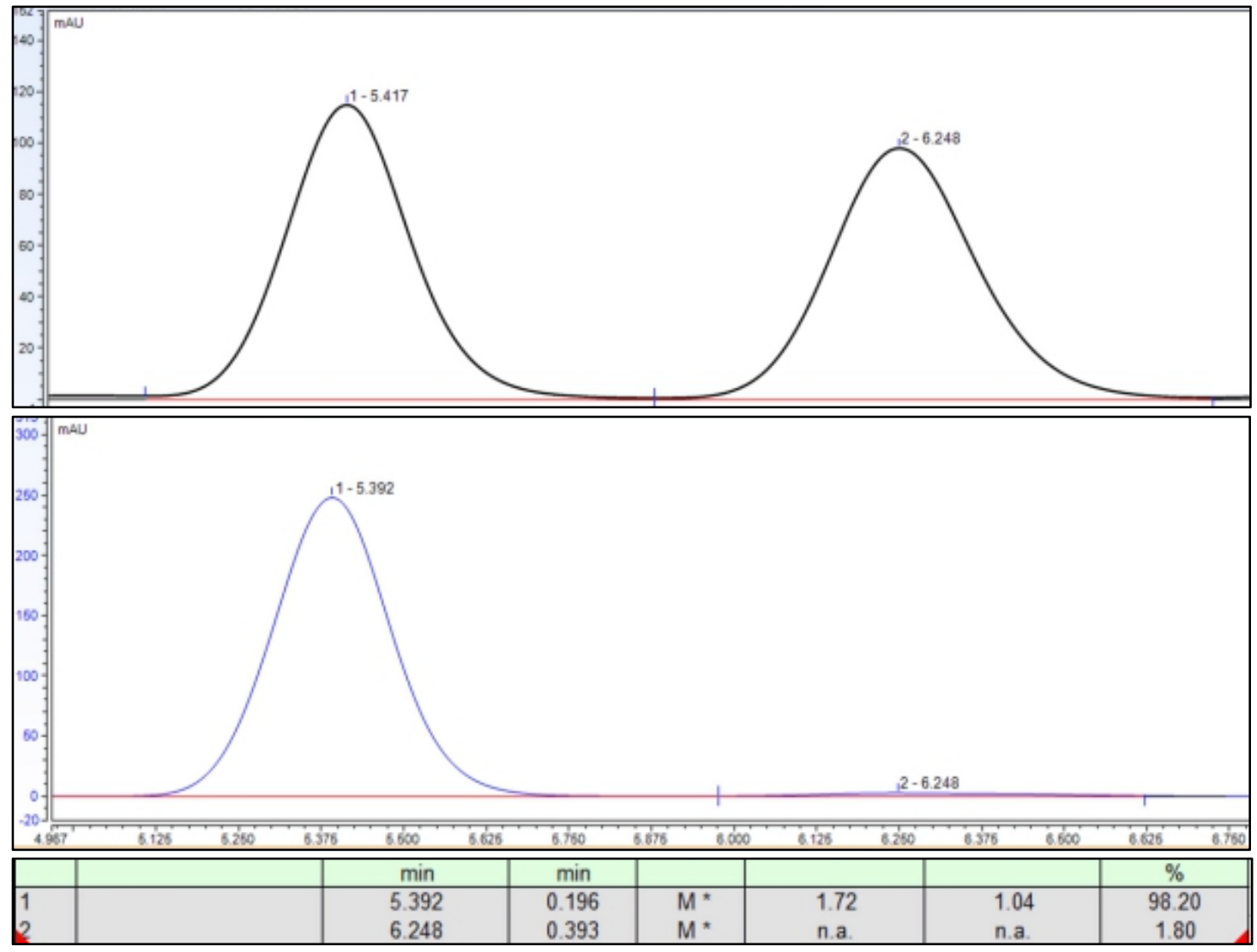

Figure S10. HPLC chromatogram of chiral product $\mathbf{2 j}$. 


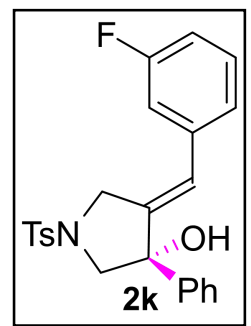

2k: yellow oil, $(81.3 \mathrm{mg}, 96 \%), 99: 1$ er. $[\alpha]_{\mathrm{D}}{ }^{25}=-6.8\left(\mathrm{c}=1.0, \mathrm{CH}_{2} \mathrm{Cl}_{2}\right)$; Enantiomeric ratio was determined by chiral HPLC. Chiralpark AD-H, $25{ }^{\circ} \mathrm{C}$, flow rate: $1 \mathrm{~mL} / \mathrm{min}$, hexanes/isopropanol: 25/75, $254 \mathrm{~nm}, 10.77 \min (S), 11.82$ $\min (R)$.

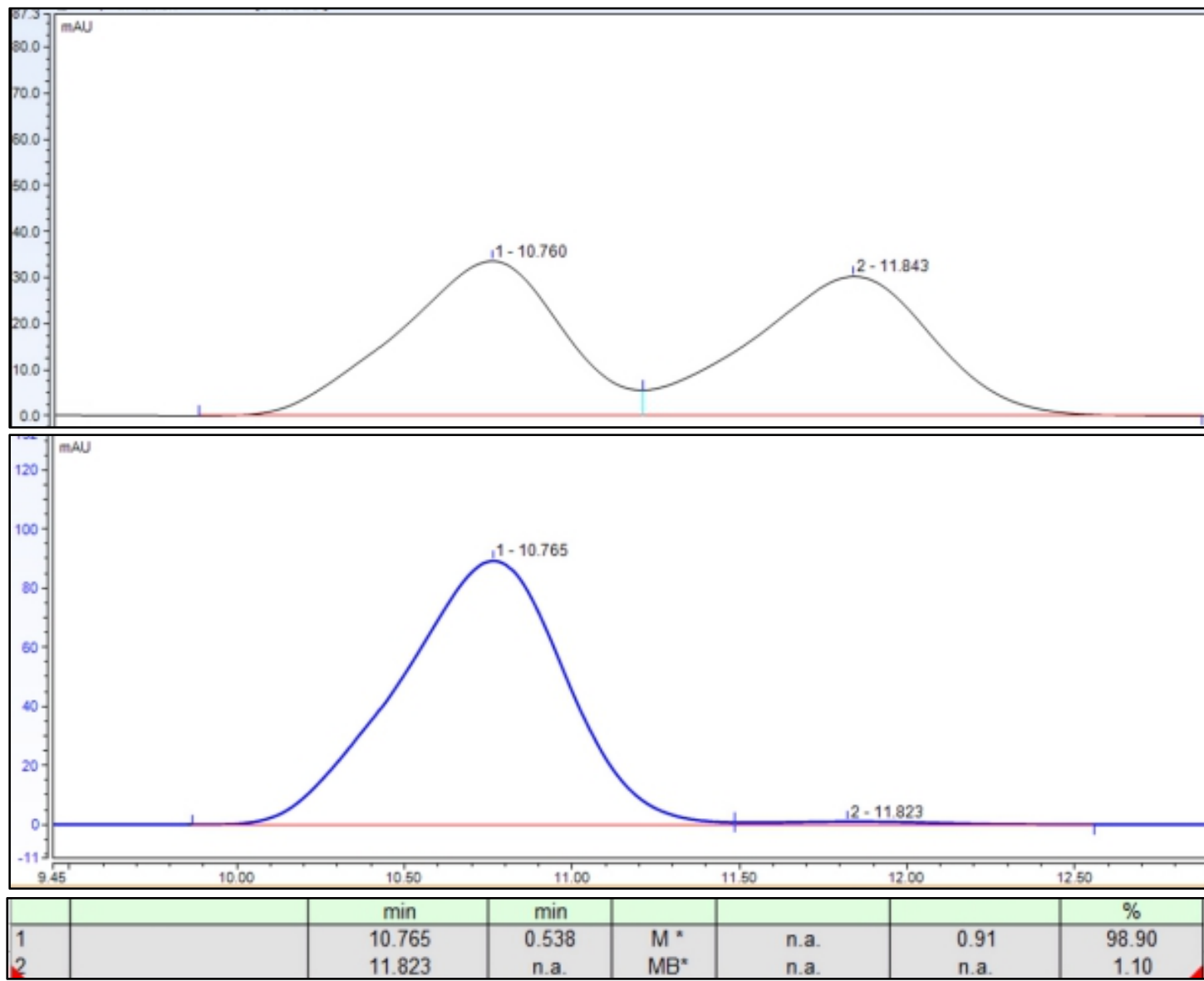

Figure Sl1. HPLC chromatogram of chiral product $\mathbf{2 k}$. 


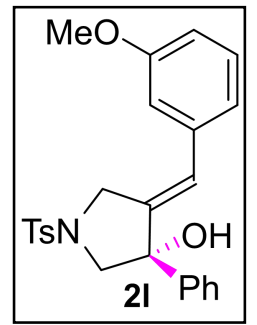

2I: yellow oil, $(83.6 \mathrm{mg}, 96 \%), 99: 1$ er. $[\alpha]_{\mathrm{D}}{ }^{25}=-22.6\left(\mathrm{c}=1.0, \mathrm{CH}_{2} \mathrm{Cl}_{2}\right)$; Enantiomeric ratio was determined by chiral HPLC. Chiralpark AD-H, $25{ }^{\circ} \mathrm{C}$, flow rate: $1 \mathrm{~mL} / \mathrm{min}$, hexanes/isopropanol: $15 / 85,254 \mathrm{~nm}, 6.27 \mathrm{~min}(S), 7.60$ $\min (R)$.
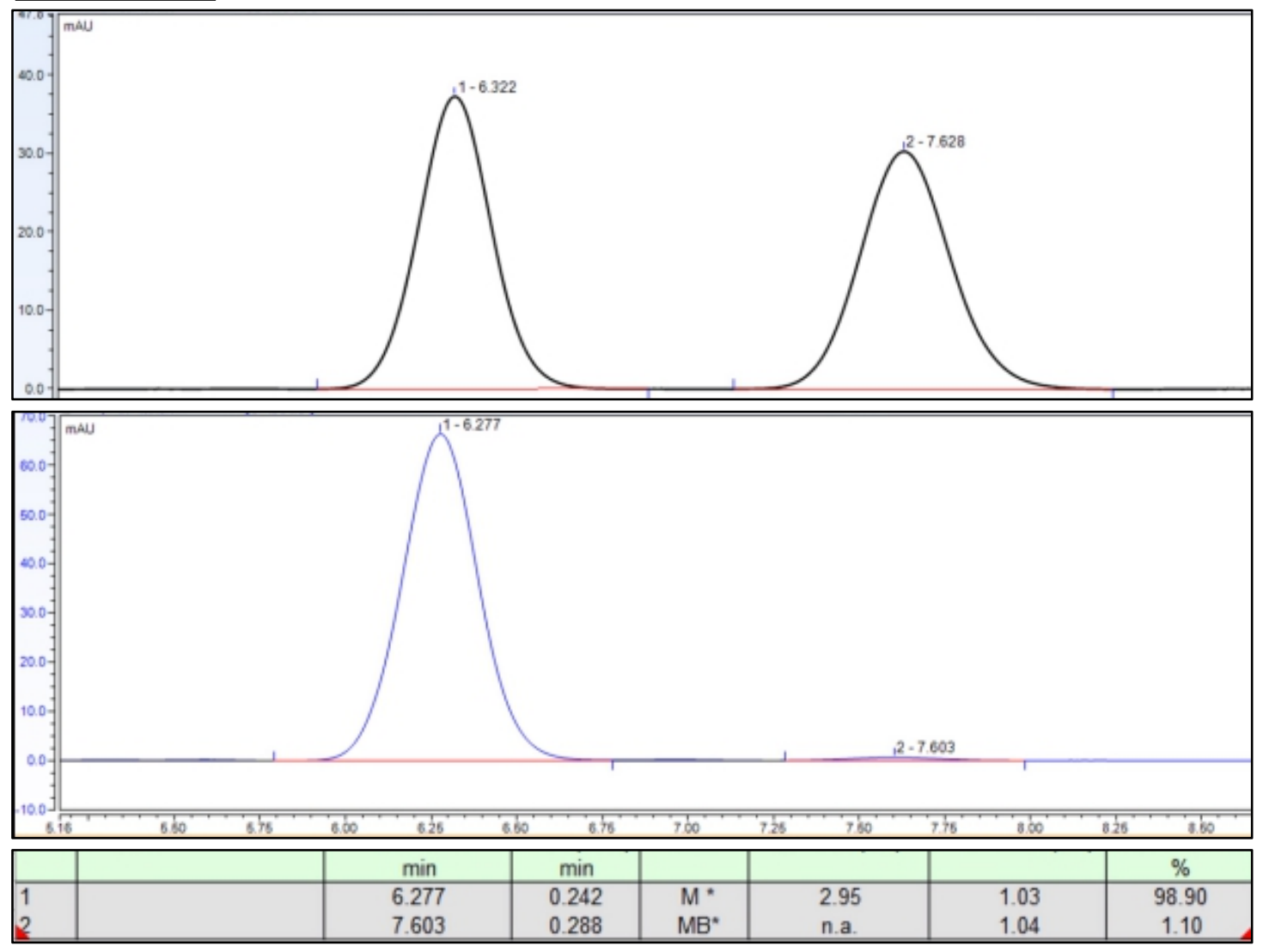

Figure Sl2. HPLC chromatogram of chiral product 21. 


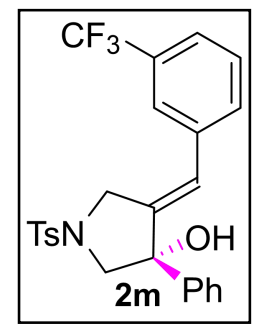

2m: white solid, $(68.1 \mathrm{mg}, 72 \%), 97: 3$ er. $[\alpha]_{\mathrm{D}}{ }^{25}=-18.6\left(\mathrm{c}=1.0, \mathrm{CH}_{2} \mathrm{Cl}_{2}\right)$; Enantiomeric ratio was determined by chiral HPLC. Chiralpark AD-H, $25{ }^{\circ} \mathrm{C}$, flow rate: $0.5 \mathrm{~mL} / \mathrm{min}$, hexanes/isopropanol: 15/85, $254 \mathrm{~nm}, 4.10 \mathrm{~min}(S), 4.77$ $\min (R)$.
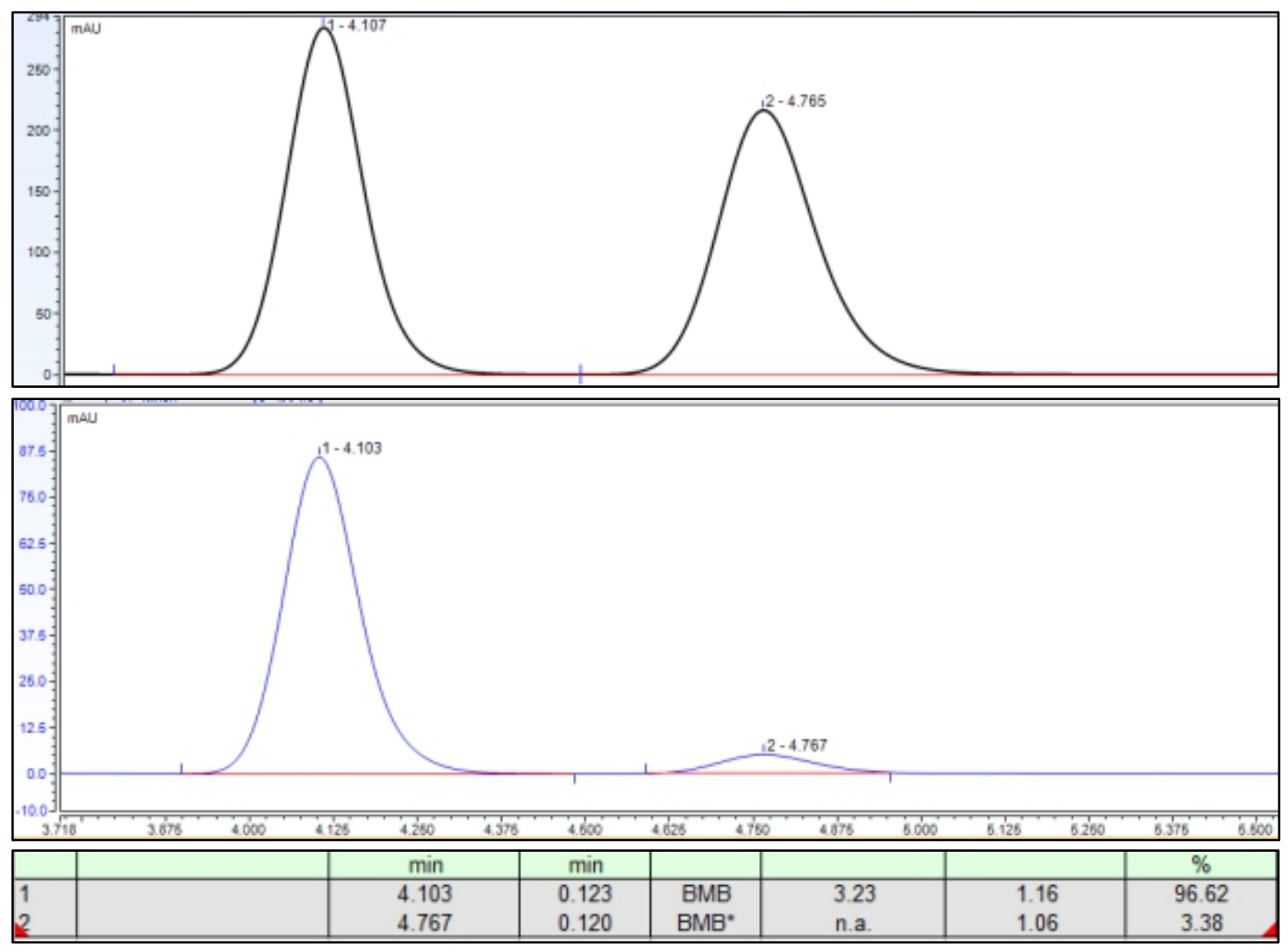

Figure S13. HPLC chromatogram of chiral product $\mathbf{2 m}$. 


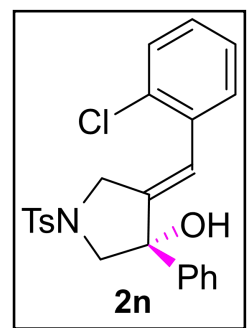

2n: yellow oil, $(63.3 \mathrm{mg}, 72 \%), 92: 8$ er. $[\alpha]_{\mathrm{D}}^{25}=-12.9\left(\mathrm{c}=1.0, \mathrm{CH}_{2} \mathrm{Cl}_{2}\right)$; Enantiomeric ratio was determined by chiral HPLC. Chiralpark AD-H, $25{ }^{\circ} \mathrm{C}$, flow rate: $1 \mathrm{~mL} / \mathrm{min}$, hexanes/isopropanol: 15/85, $254 \mathrm{~nm}, 4.74 \mathrm{~min}(S), 6.96$ $\min (R)$.
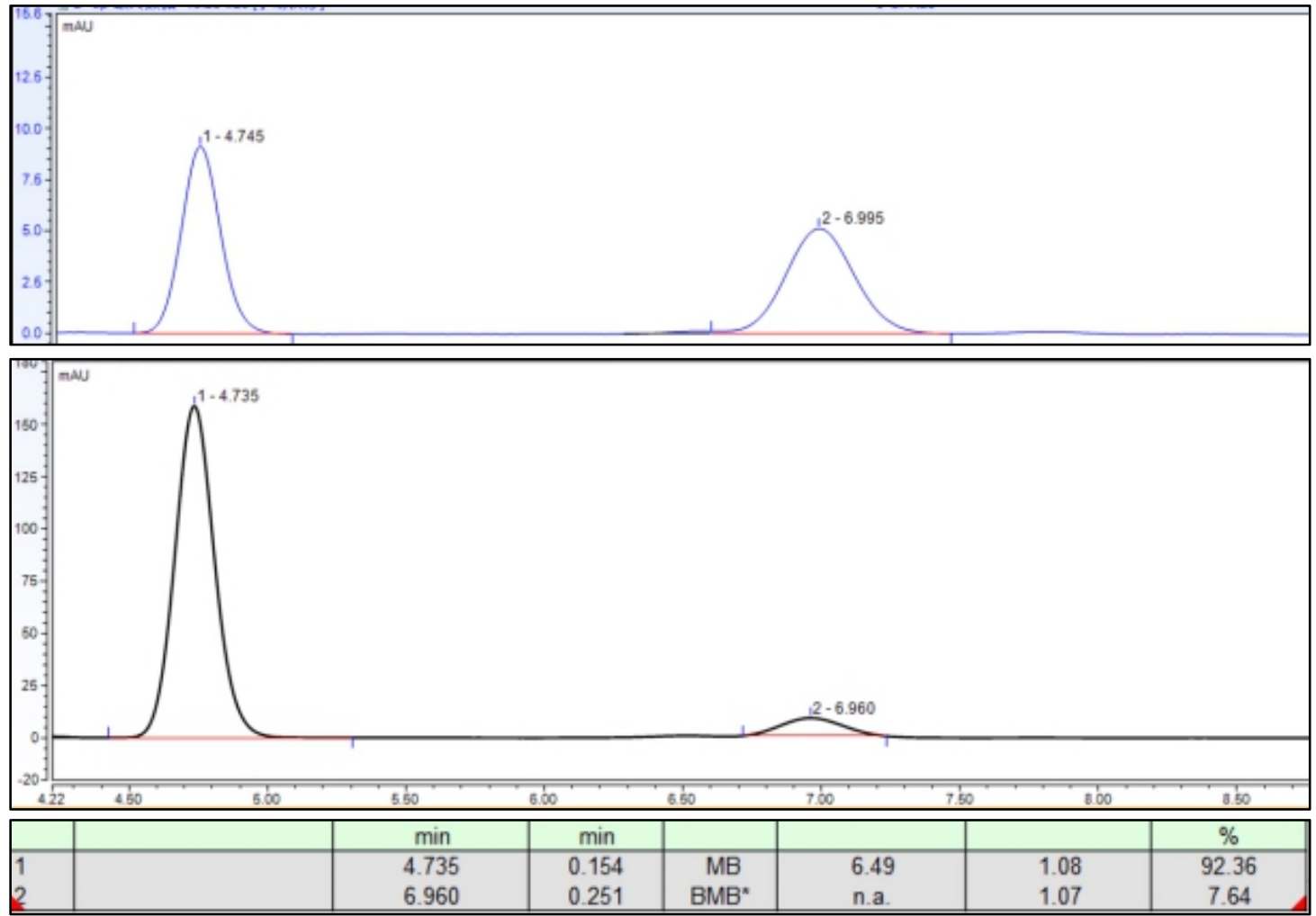

Figure SI4. HPLC chromatogram of chiral product $\mathbf{2 n}$. 


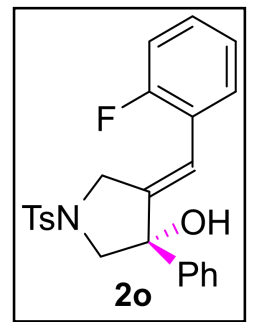

2o: white solid, $(65.2 \mathrm{mg}, 77 \%),>99: 1$ er. $[\alpha]_{\mathrm{D}}{ }^{25}=-21.1\left(\mathrm{c}=1.0, \mathrm{CH}_{2} \mathrm{Cl}_{2}\right)$; Enantiomeric ratio was determined by chiral HPLC. Chiralpark AD-H, $25{ }^{\circ} \mathrm{C}$, flow rate: $1 \mathrm{~mL} / \mathrm{min}$, hexanes/isopropanol: 15/85, $254 \mathrm{~nm}, 5.26 \mathrm{~min}(S), 6.57$ $\min (R)$.
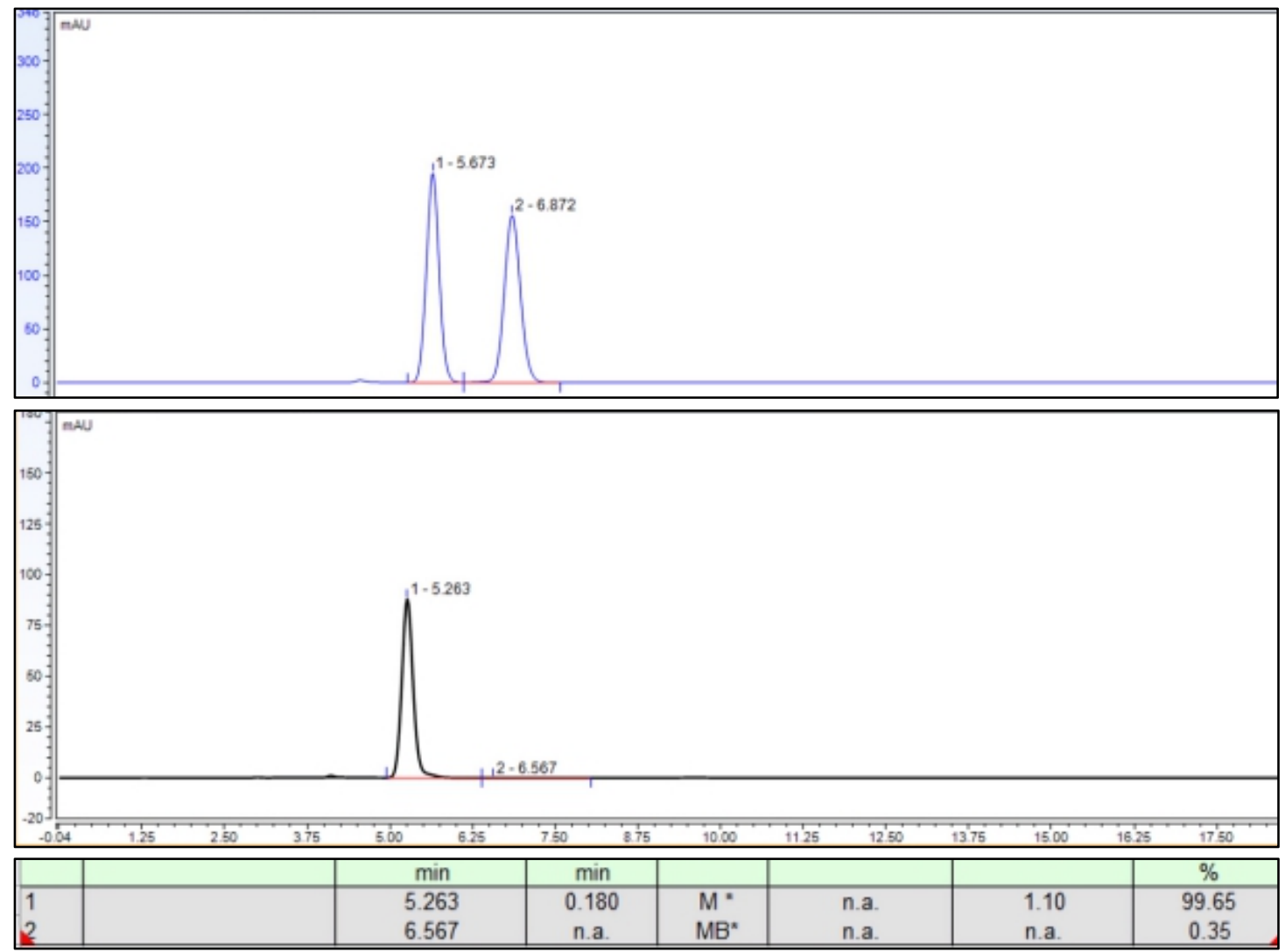

Figure S15. HPLC chromatogram of chiral product 20. 

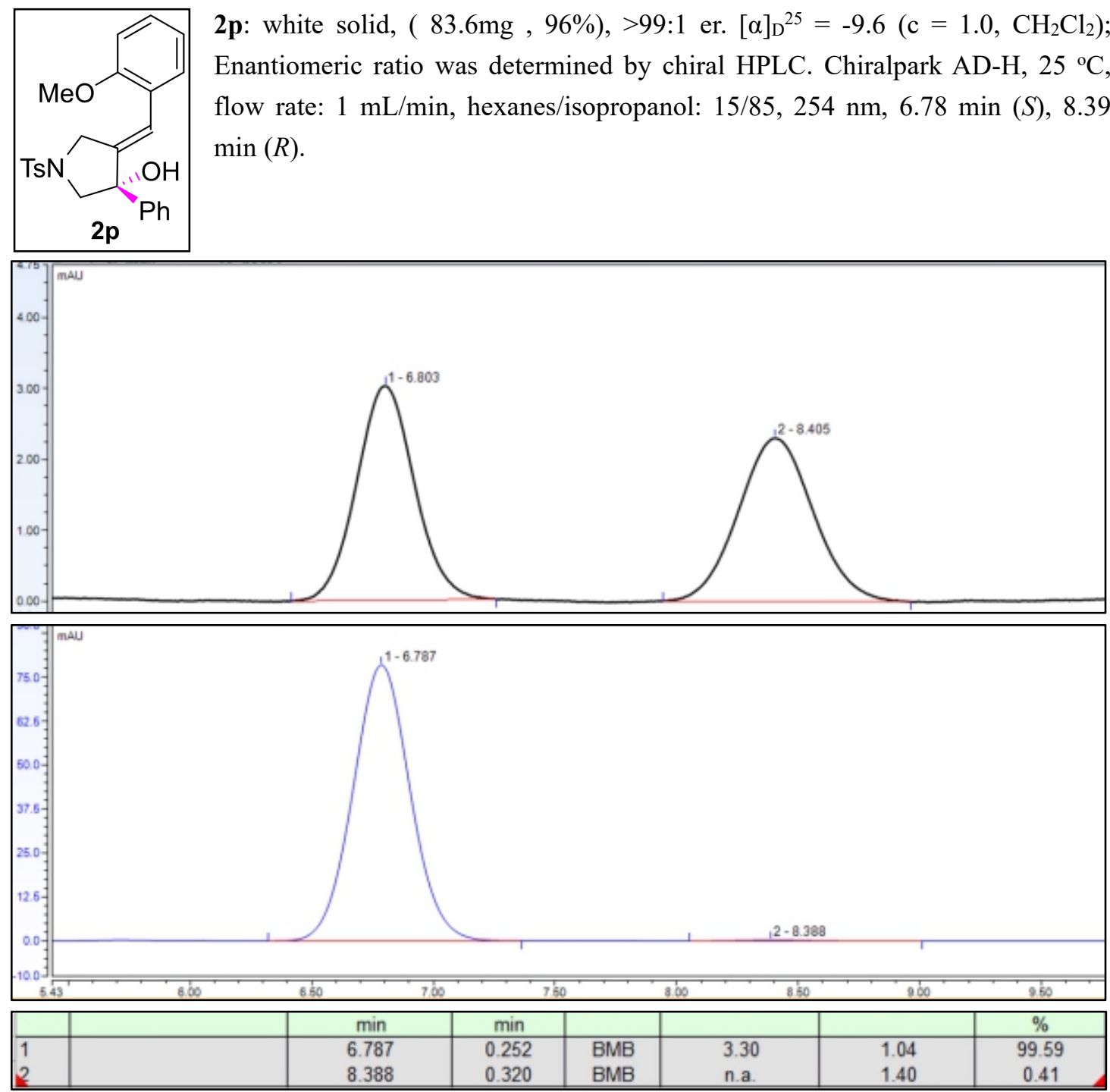

Figure Sl6. HPLC chromatogram of chiral product $\mathbf{2 p}$. 


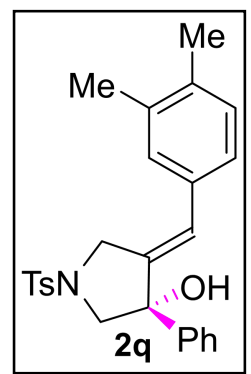

2q: white solid, $(79.7 \mathrm{mg}, 92 \%), 95: 5 \mathrm{er},[\alpha]_{\mathrm{D}}{ }^{25}=-24.0\left(\mathrm{c}=1.0, \mathrm{CH}_{2} \mathrm{Cl}_{2}\right)$; Enantiomeric ratio was determined by chiral HPLC. Chiralpark AD-H, $25{ }^{\circ} \mathrm{C}$, flow rate: $0.5 \mathrm{~mL} / \mathrm{min}$, hexanes/isopropanol: 15/85, $254 \mathrm{~nm}, 12.027 \mathrm{~min}(S)$, $14.692 \min (R)$.
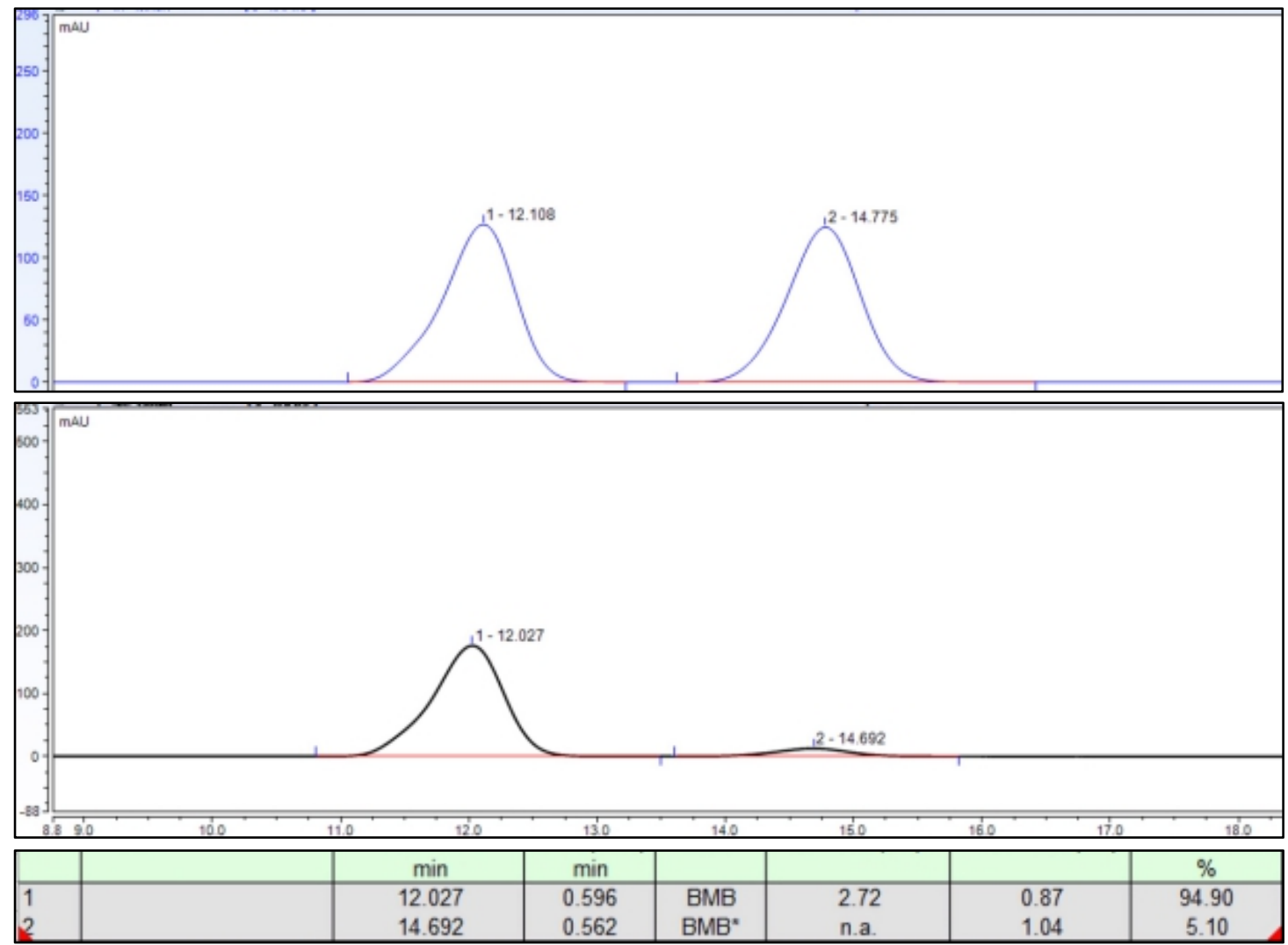

Figure S17. HPLC chromatogram of chiral product 2q. 


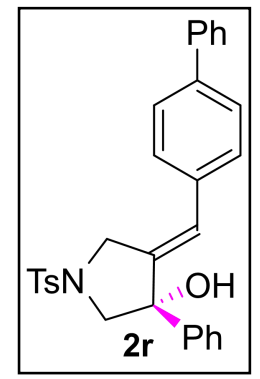

2r: white solid, $(70.3 \mathrm{mg}, 73 \%), 98: 2$ er. $[\alpha]_{\mathrm{D}}^{25}=-38.7\left(\mathrm{c}=1.0, \mathrm{CH}_{2} \mathrm{Cl}_{2}\right)$; Enantiomeric ratio was determined by chiral HPLC. Chiralpark AD-H, $25{ }^{\circ} \mathrm{C}$, flow rate: $0.5 \mathrm{~mL} / \mathrm{min}$, hexanes/isopropanol: 15/85, $254 \mathrm{~nm}, 9.66 \mathrm{~min}(R), 11.88$ $\min (S)$.
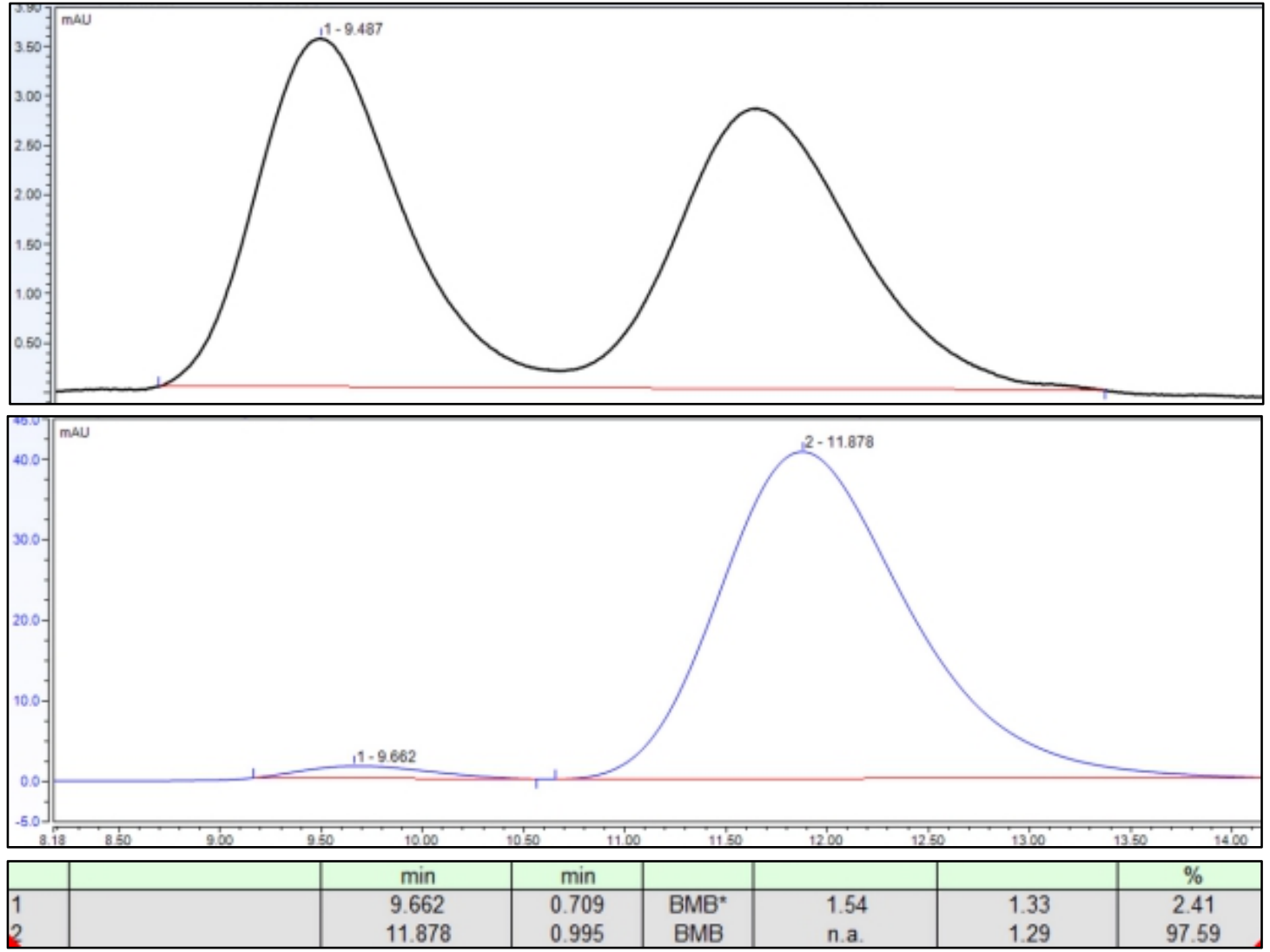

Figure S18. HPLC chromatogram of chiral product $\mathbf{2 r}$. 


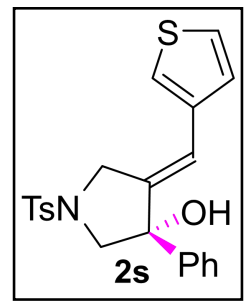

2s: white solid, $(71.5 \mathrm{mg}, 87 \%), 99: 1$ er. $[\alpha]_{\mathrm{D}}{ }^{25}=-18.8\left(\mathrm{c}=1.0, \mathrm{CH}_{2} \mathrm{Cl}_{2}\right)$; Enantiomeric ratio was determined by chiral HPLC. Chiralpark AD-H, $25{ }^{\circ} \mathrm{C}$, flow rate: $0.5 \mathrm{~mL} / \mathrm{min}$, hexanes/isopropanol: $15 / 85,254 \mathrm{~nm}, 5.35 \mathrm{~min}(R), 6.22$ $\min (S)$.
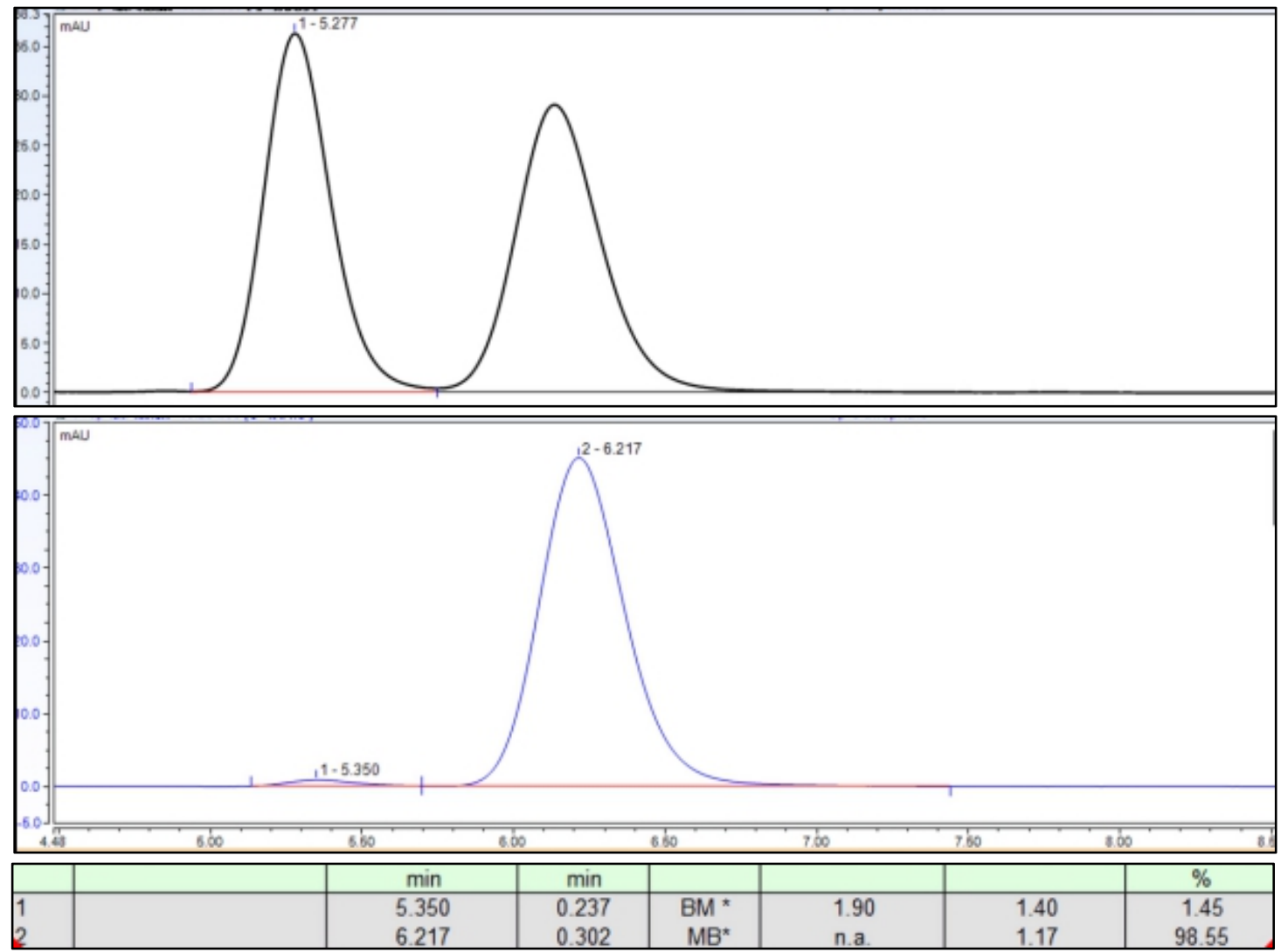

Figure S19. HPLC chromatogram of chiral product $\mathbf{2 s .}$ 


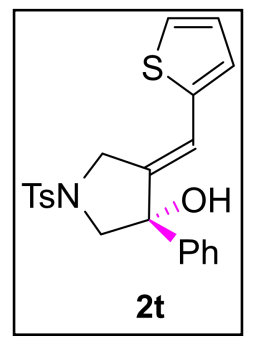

2t: colourless oil, $(66.6 \mathrm{mg}, 81 \%), 99: 1$ er. $[\alpha] \mathrm{D}^{25}=-18.0\left(\mathrm{c}=1.0, \mathrm{CH}_{2} \mathrm{Cl}_{2}\right)$; Enantiomeric ratio was determined by chiral HPLC. Chiralpark AD-H, $25{ }^{\circ} \mathrm{C}$, flow rate: $0.5 \mathrm{~mL} / \mathrm{min}$, hexanes/isopropanol: $15 / 85,254 \mathrm{~nm}, 4.88 \mathrm{~min}(R), 6.16$ $\min (S)$.
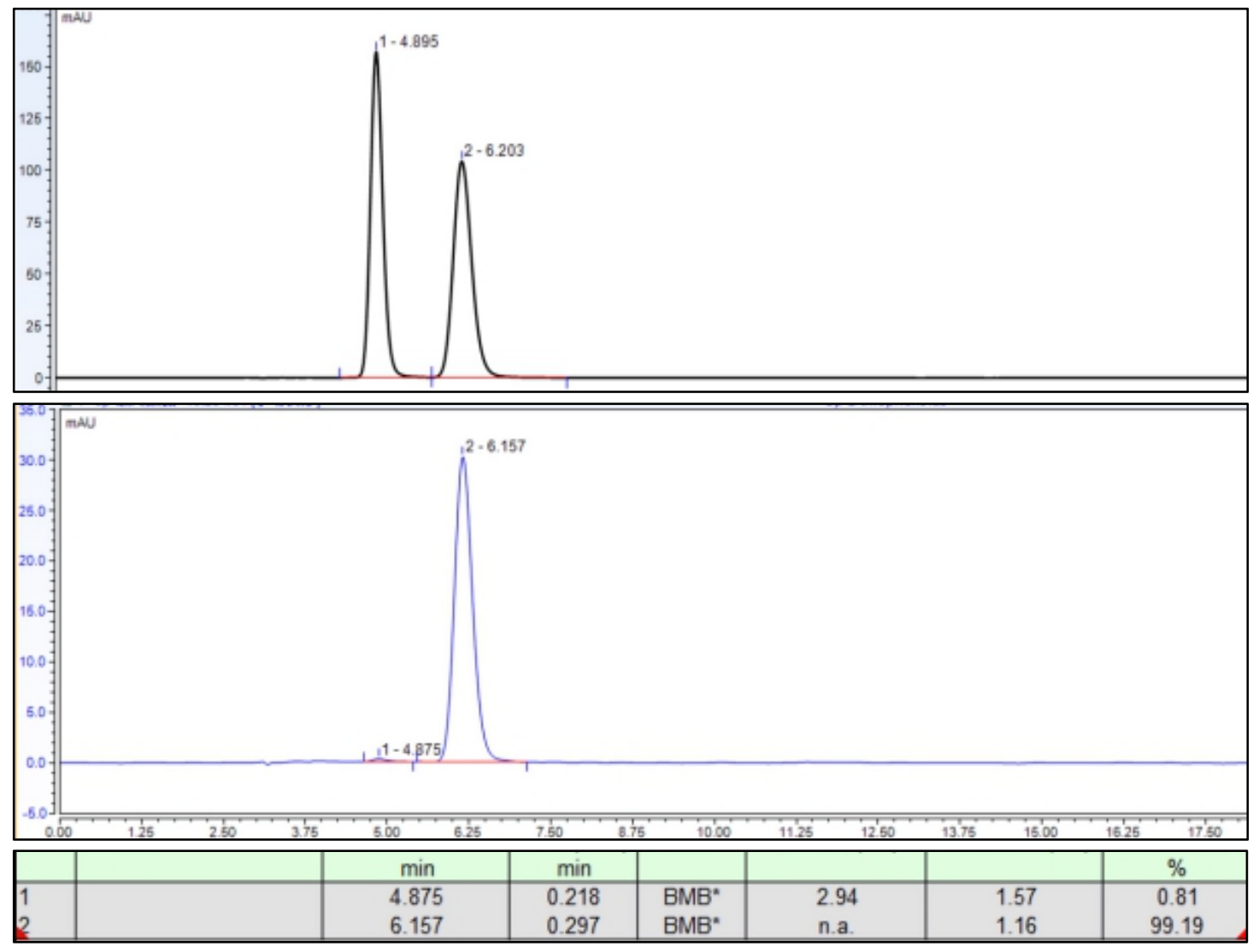

Figure S20. HPLC chromatogram of chiral product $\mathbf{2 t}$. 


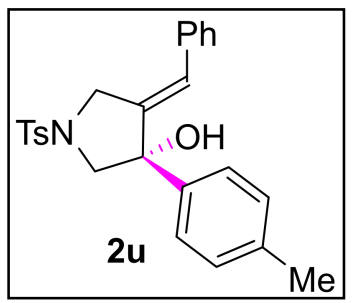

2u: colorless oil, $(77.2 \mathrm{mg}, 92 \%),>99: 1 \mathrm{er},[\alpha]_{\mathrm{D}}{ }^{25}=-24.3(\mathrm{c}=1.0$, $\mathrm{CH}_{2} \mathrm{Cl}_{2}$ ); Enantiomeric ratio was determined by chiral HPLC. Chiralpark AD-H, $25{ }^{\circ} \mathrm{C}$, flow rate: $0.5 \mathrm{~mL} / \mathrm{min}$, hexanes/isopropanol: 15/85, $254 \mathrm{~nm}, 10.80 \mathrm{~min}(S), 12.22 \min (R)$.
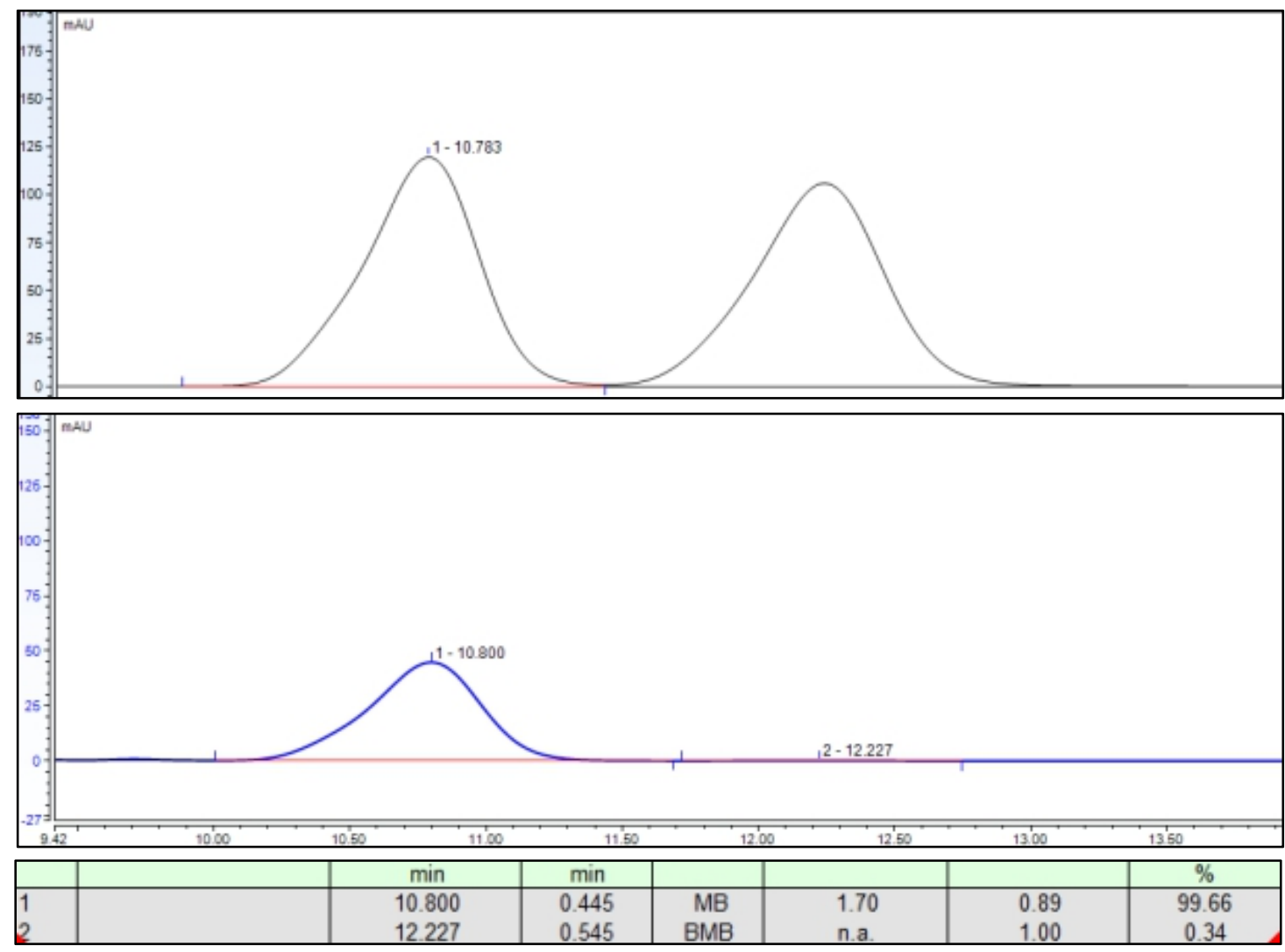

Figure S21. HPLC chromatogram of chiral product $\mathbf{2 u}$. 


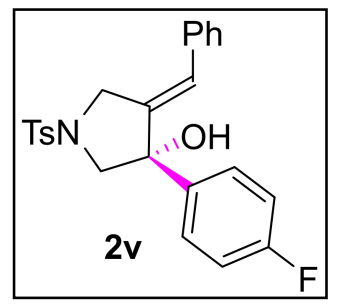

2v: colorless oil, (78.0mg, 92\%), > 99:1 er. $[\alpha]_{\mathrm{D}}{ }^{25}=-23.7\left(\mathrm{c}=1.0, \mathrm{CH}_{2} \mathrm{Cl}_{2}\right)$ Enantiomeric ratio was determined by chiral HPLC. Chiralpark AD-H, 25 ${ }^{\circ} \mathrm{C}$, flow rate: $0.5 \mathrm{~mL} / \mathrm{min}$, hexanes/isopropanol: $15 / 85,254 \mathrm{~nm}, 5.25 \mathrm{~min}$ $(S), 5.58 \min (R)$.
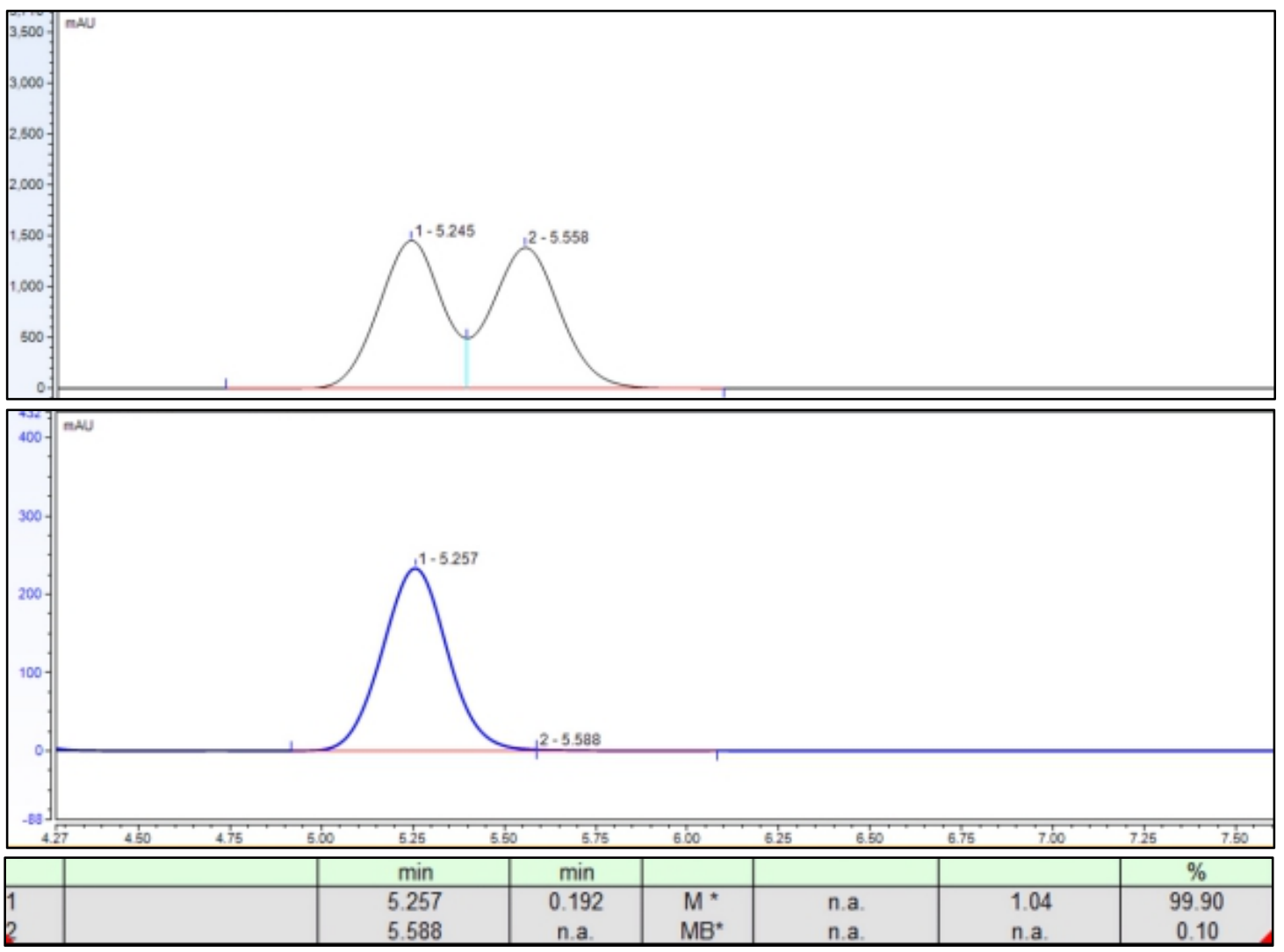

Figure S22. HPLC chromatogram of chiral product 2v. 


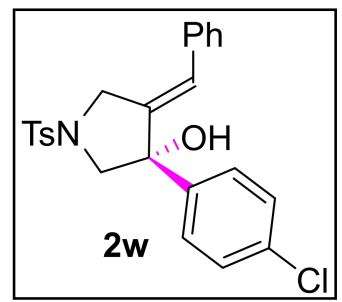

2w: colorless oil, $(72.1 \mathrm{mg}, 82 \%),>99: 1$ er, $[\alpha]_{\mathrm{D}}^{25}=-25.06(\mathrm{c}=1.0$, $\mathrm{CH}_{2} \mathrm{Cl}_{2}$ ); Enantiomeric ratio was determined by chiral HPLC. Chiralpark $\mathrm{AD}-\mathrm{H}, 25^{\circ} \mathrm{C}$, flow rate: $0.5 \mathrm{~mL} / \mathrm{min}$, hexanes/isopropanol: 30/70, $254 \mathrm{~nm}$, $6.07 \min (S), 6.44 \min (R)$.
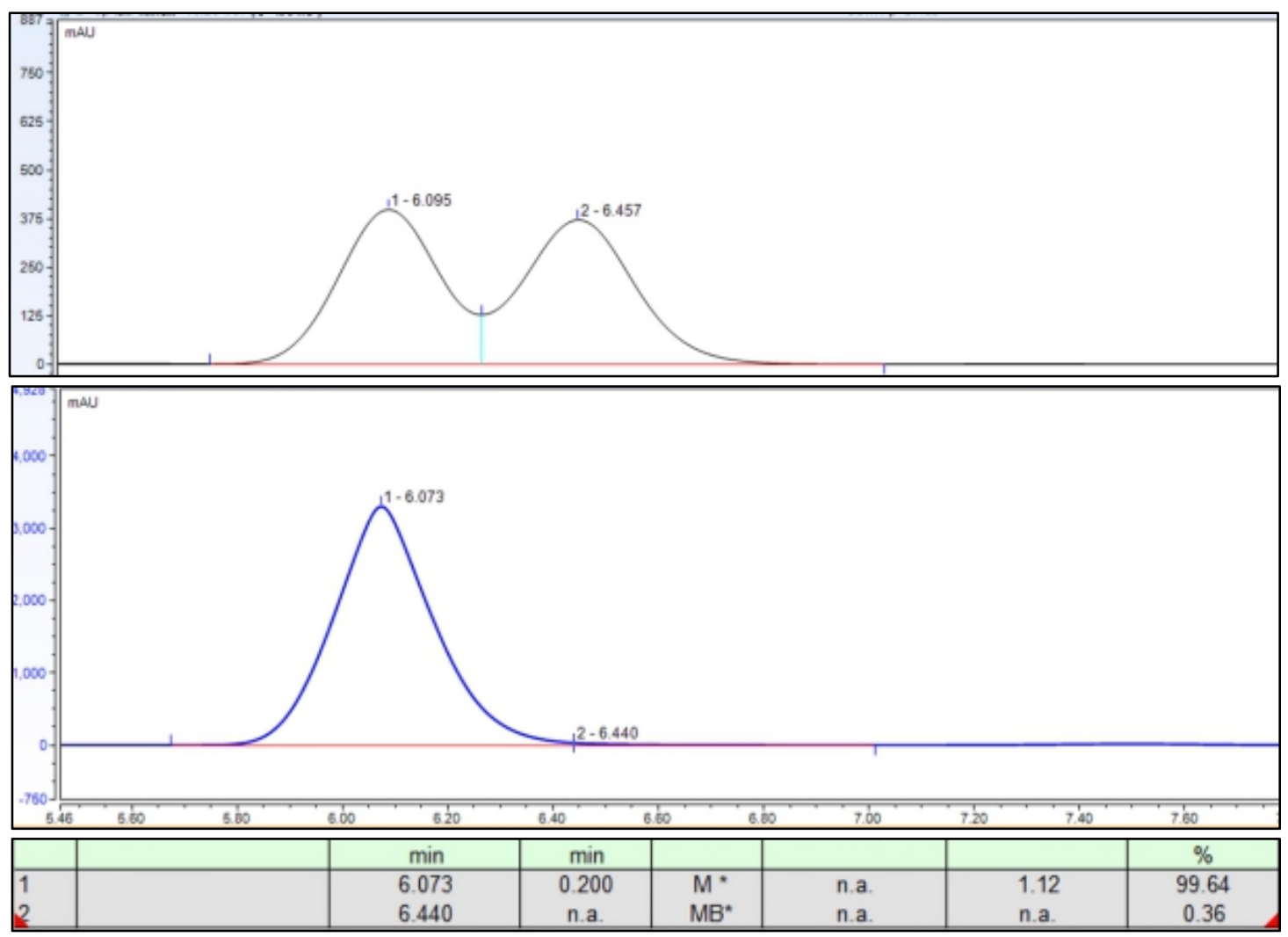

Figure S23. HPLC chromatogram of chiral product 2w. 


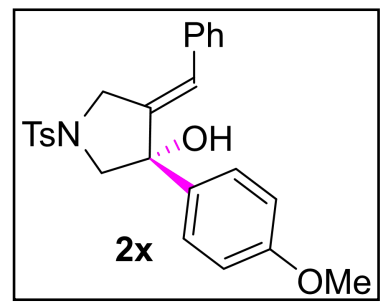

2x: white solid, $(81.8 \mathrm{mg}, 94 \%), 99: 1 \mathrm{er},[\alpha]_{\mathrm{D}}^{25}=-36.32(\mathrm{c}=1.0$, $\mathrm{CH}_{2} \mathrm{Cl}_{2}$ ); Enantiomeric ratio was determined by chiral HPLC. Chiralpark AD-H, $25{ }^{\circ} \mathrm{C}$, flow rate: $0.5 \mathrm{~mL} / \mathrm{min}$, hexanes/isopropanol: 25/75, $254 \mathrm{~nm}, 16.85 \min (R), 21.99 \min (S)$.
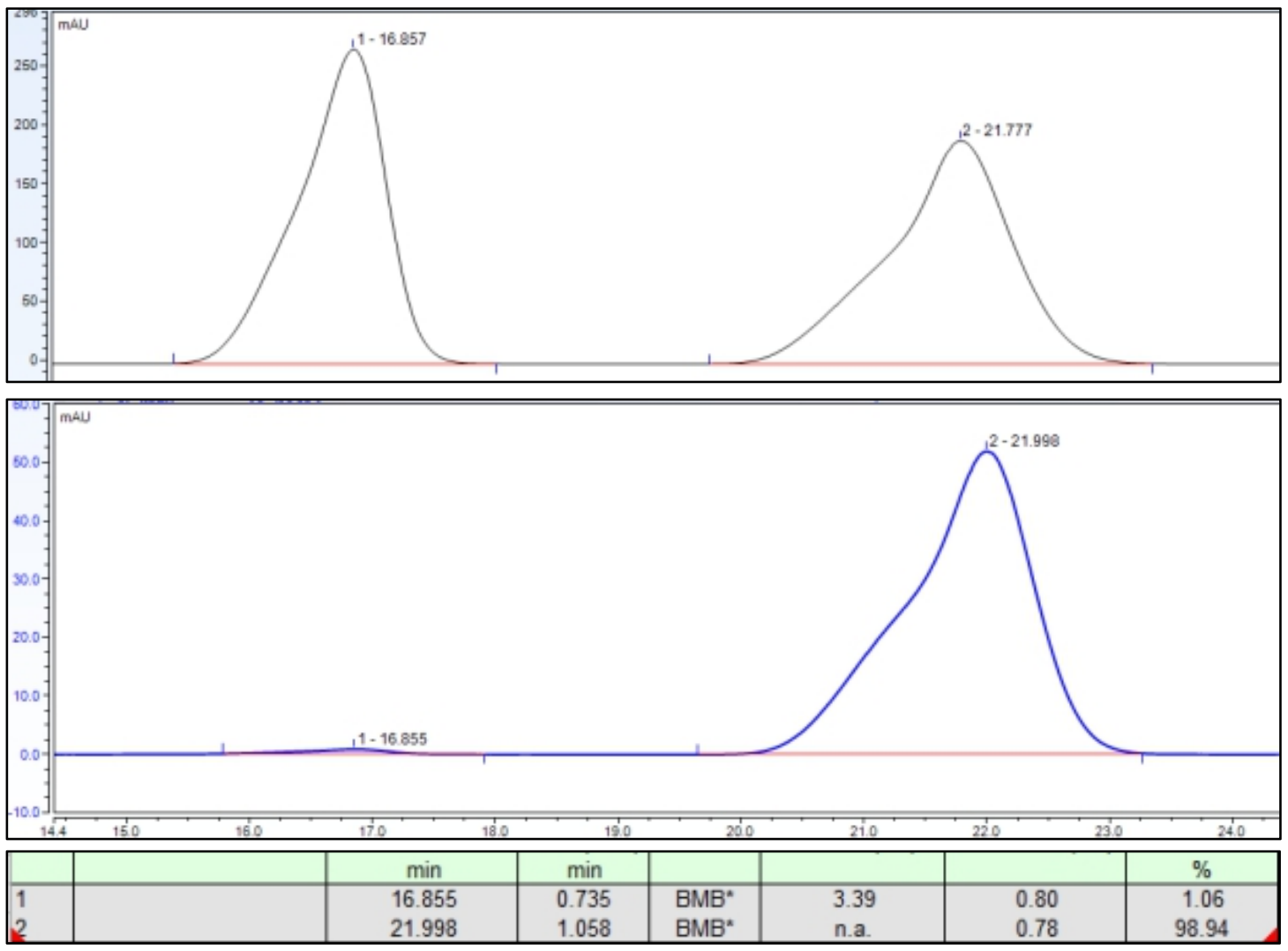

Figure S24. HPLC chromatogram of chiral product 2x. 


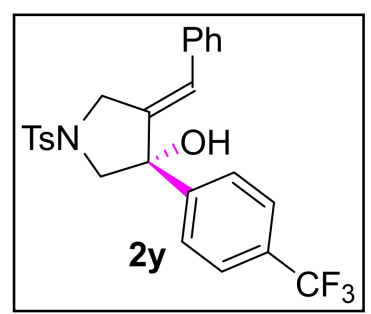

2y: yellow oil, $(89.9 \mathrm{mg}, 95 \%),>99: 1$ er, $[\alpha]_{\mathrm{D}}{ }^{25}=-18.19(\mathrm{c}=1.0$, $\mathrm{CH}_{2} \mathrm{Cl}_{2}$ ); Enantiomeric ratio was determined by chiral HPLC. Chiralpark AD-H, $25{ }^{\circ} \mathrm{C}$, flow rate: $0.5 \mathrm{~mL} / \mathrm{min}$, hexanes/isopropanol: 30/70, $254 \mathrm{~nm}, 4.56 \mathrm{~min}(S), 4.99 \min (R)$.
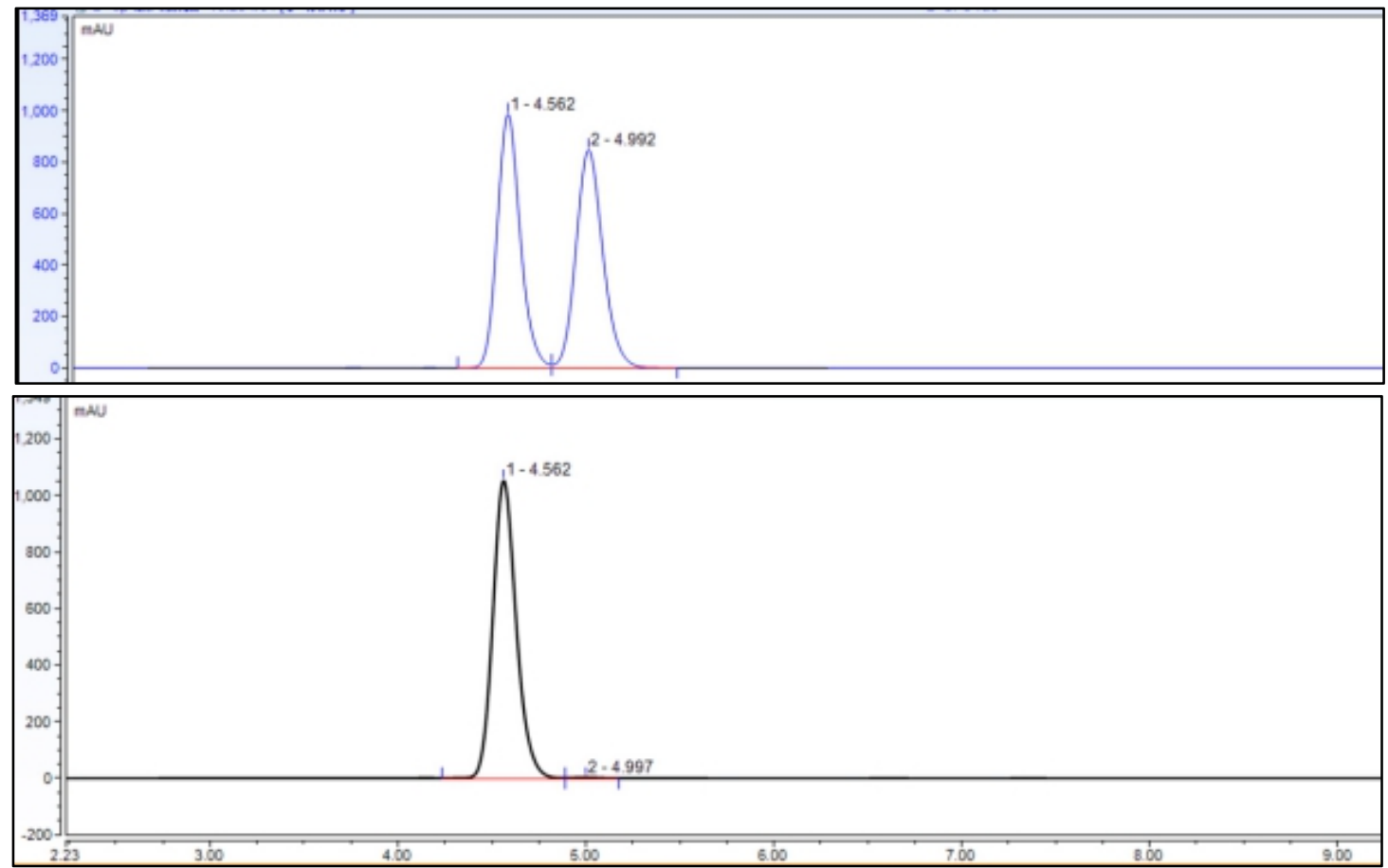

\begin{tabular}{|l|c|c|c|c|c|c|c|}
\hline & & $\min$ & $\min$ & & & & $\%$ \\
\hline 1 & & 4.562 & 0.131 & $\mathbf{M}^{*}$ & 1.70 & 1.18 & 99.58 \\
2 & 4.997 & 0.171 & $\mathbf{M}^{*}$ & n.a. & n.a. & 0.42 \\
\hline
\end{tabular}

Figure S25. HPLC chromatogram of chiral product $\mathbf{2 y}$. 


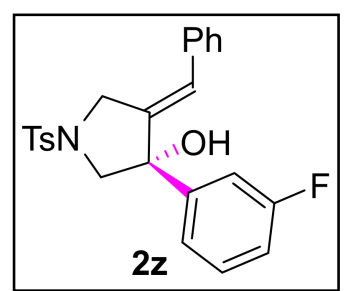

2z: colorless oil, $(73.7 \mathrm{mg}, 87 \%),>99: 1$ er. $[\alpha]_{\mathrm{D}}{ }^{25}=-11.1\left(\mathrm{c}=1.0, \mathrm{CH}_{2} \mathrm{Cl}_{2}\right)$ Enantiomeric ratio was determined by chiral HPLC. Chiralpark AD-H, 25 ${ }^{\circ} \mathrm{C}$, flow rate: $0.5 \mathrm{~mL} / \mathrm{min}$, hexanes/isopropanol: $15 / 85,254 \mathrm{~nm}, 5.23 \mathrm{~min}$ $(S), 6.70 \min (R)$.
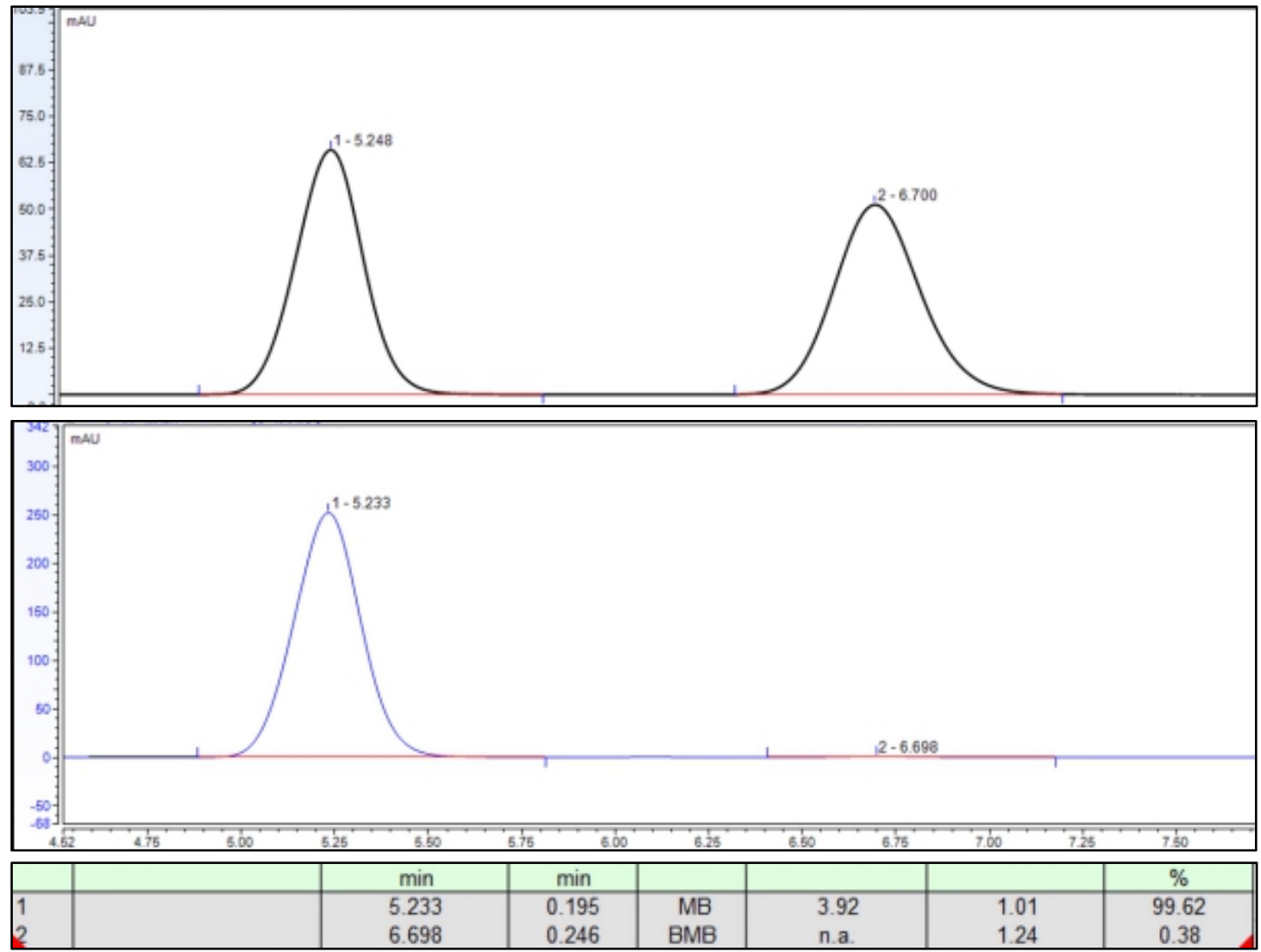

Figure S26. HPLC chromatogram of chiral product $\mathbf{2 z}$. 


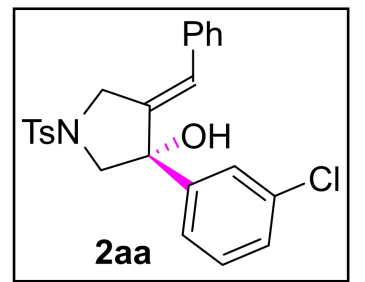

2aa: white solid, $(68.6 \mathrm{mg}, 78 \%), 99: 1 \mathrm{er},[\alpha]_{\mathrm{D}}{ }^{25}=-53.0\left(\mathrm{c}=1.0, \mathrm{CH}_{2} \mathrm{Cl}_{2}\right)$ Enantiomeric ratio was determined by chiral HPLC. Chiralpark AD-H, $25{ }^{\circ} \mathrm{C}$, flow rate: $0.5 \mathrm{~mL} / \mathrm{min}$, hexanes/isopropanol: 15/85, $254 \mathrm{~nm}, 6.60$ $\min (S), 7.50 \min (R)$.

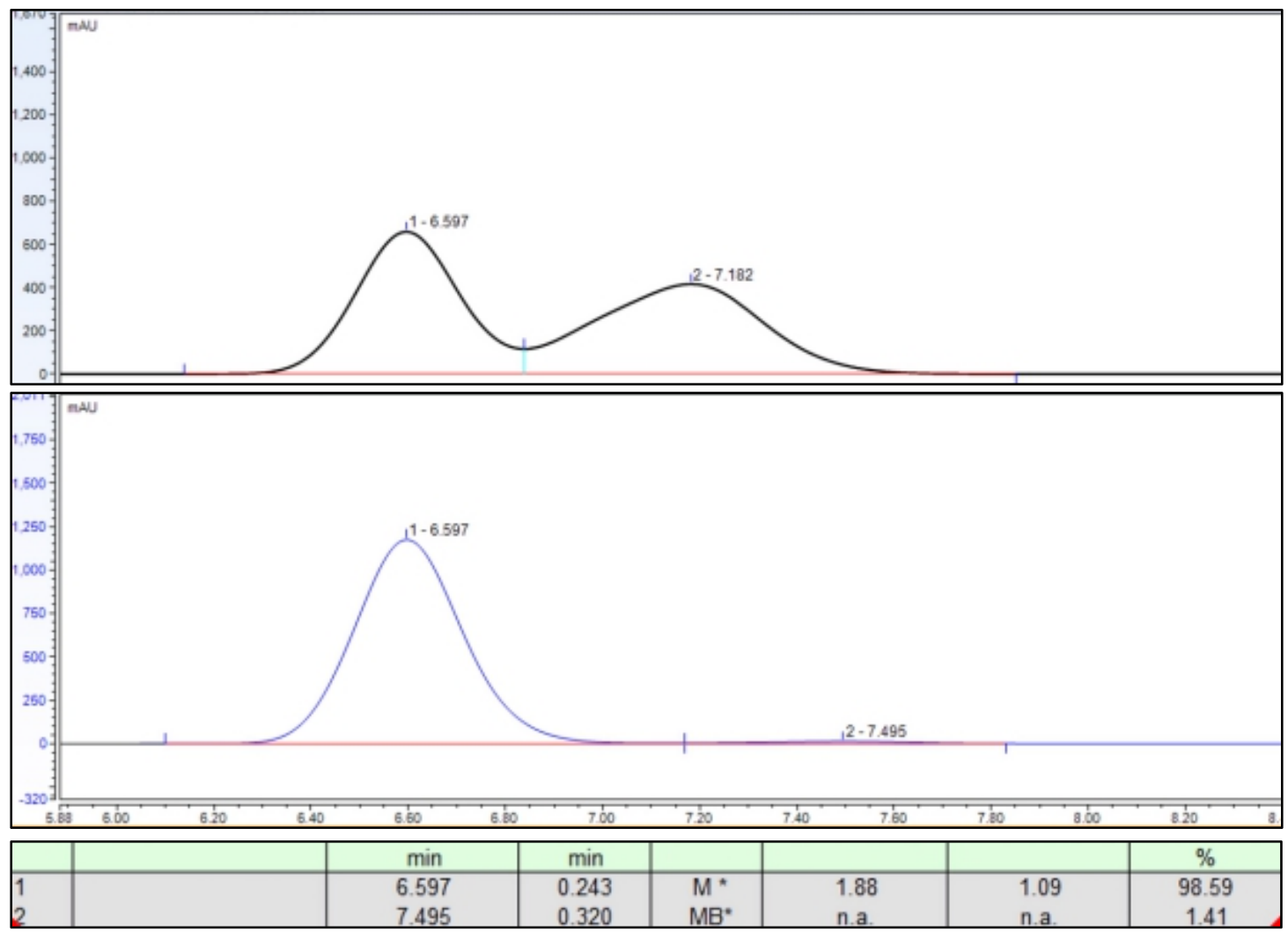

Figure S27. HPLC chromatogram of chiral product $\mathbf{2 a a}$. 


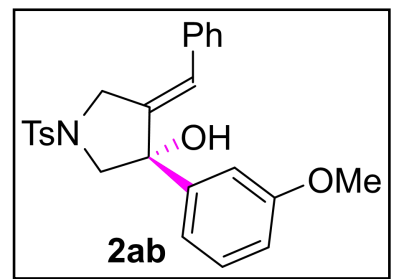

2ab: yellow oil, (74.1mg, 85\%), 98:2 er. $[\alpha]_{\mathrm{D}}{ }^{25}=-32.1 \quad(\mathrm{c}=$ 1.0, $\mathrm{CH}_{2} \mathrm{Cl}_{2}$ ); Enantiomeric ratio was determined by chiral HPLC. Chiralpark AD-H, $25{ }^{\circ} \mathrm{C}$, flow rate: $0.5 \mathrm{~mL} / \mathrm{min}$, hexanes/isopropanol: 25/75, $254 \mathrm{~nm}, 10.88 \mathrm{~min}(R), 13.37 \mathrm{~min}(S)$.
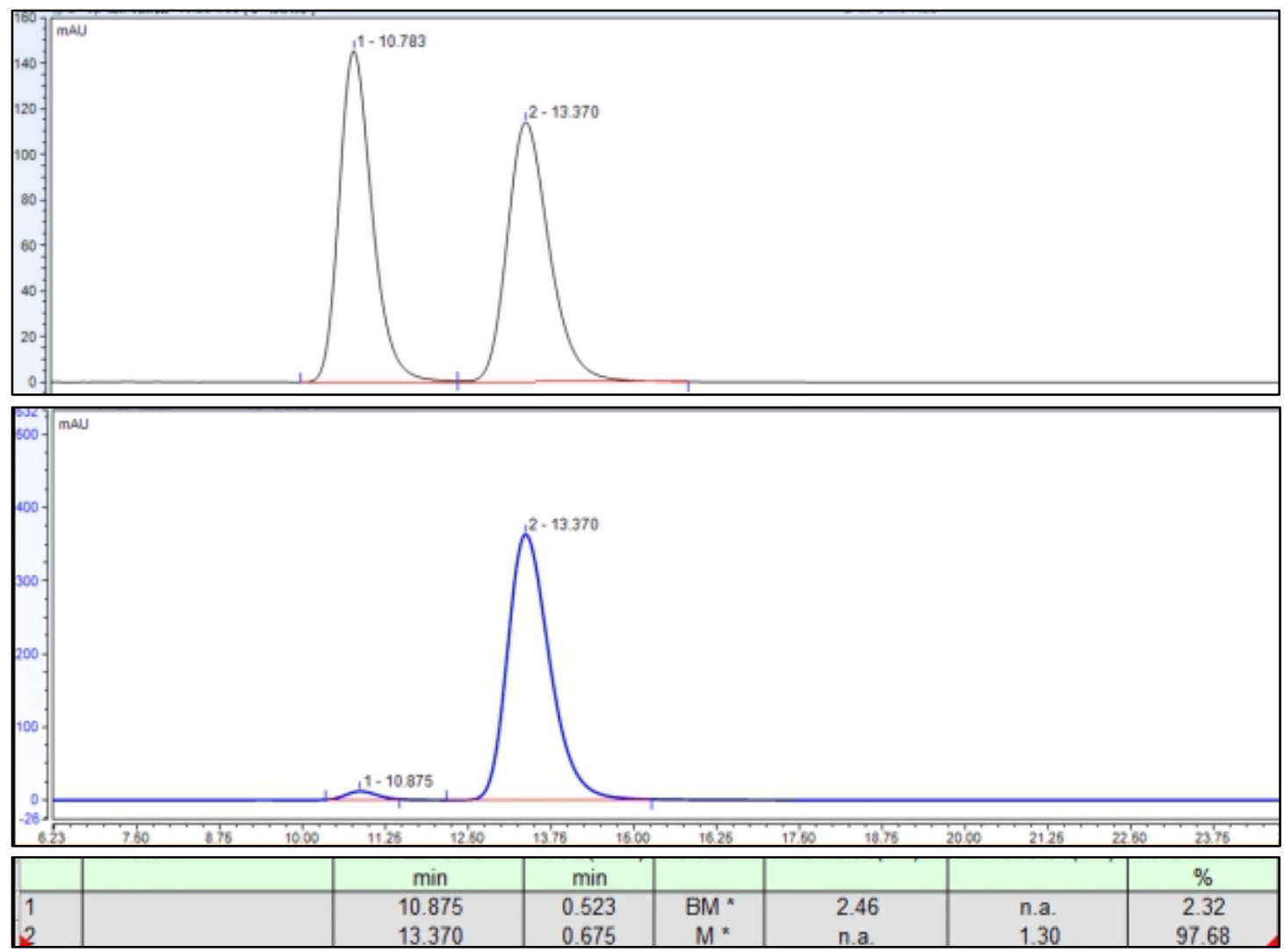

Figure S28. HPLC chromatogram of chiral product $\mathbf{2 a b}$. 


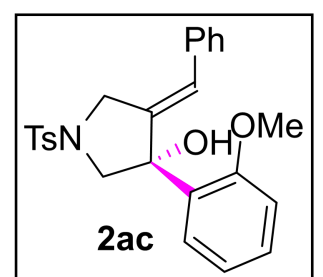

2ac: white solid, (55.7mg, 64\%), 98:2 er, $[\alpha]_{\mathrm{D}}^{25}=-19.33\left(\mathrm{c}=1.0, \mathrm{CH}_{2} \mathrm{Cl}_{2}\right)$; Enantiomeric ratio was determined by chiral HPLC. Chiralpark AD-H, 25 ${ }^{\circ} \mathrm{C}$, flow rate: $0.5 \mathrm{~mL} / \mathrm{min}$, hexanes/isopropanol: $25 / 75,254 \mathrm{~nm}, 10.88 \mathrm{~min}$ $(R), 13.37 \min (S)$.
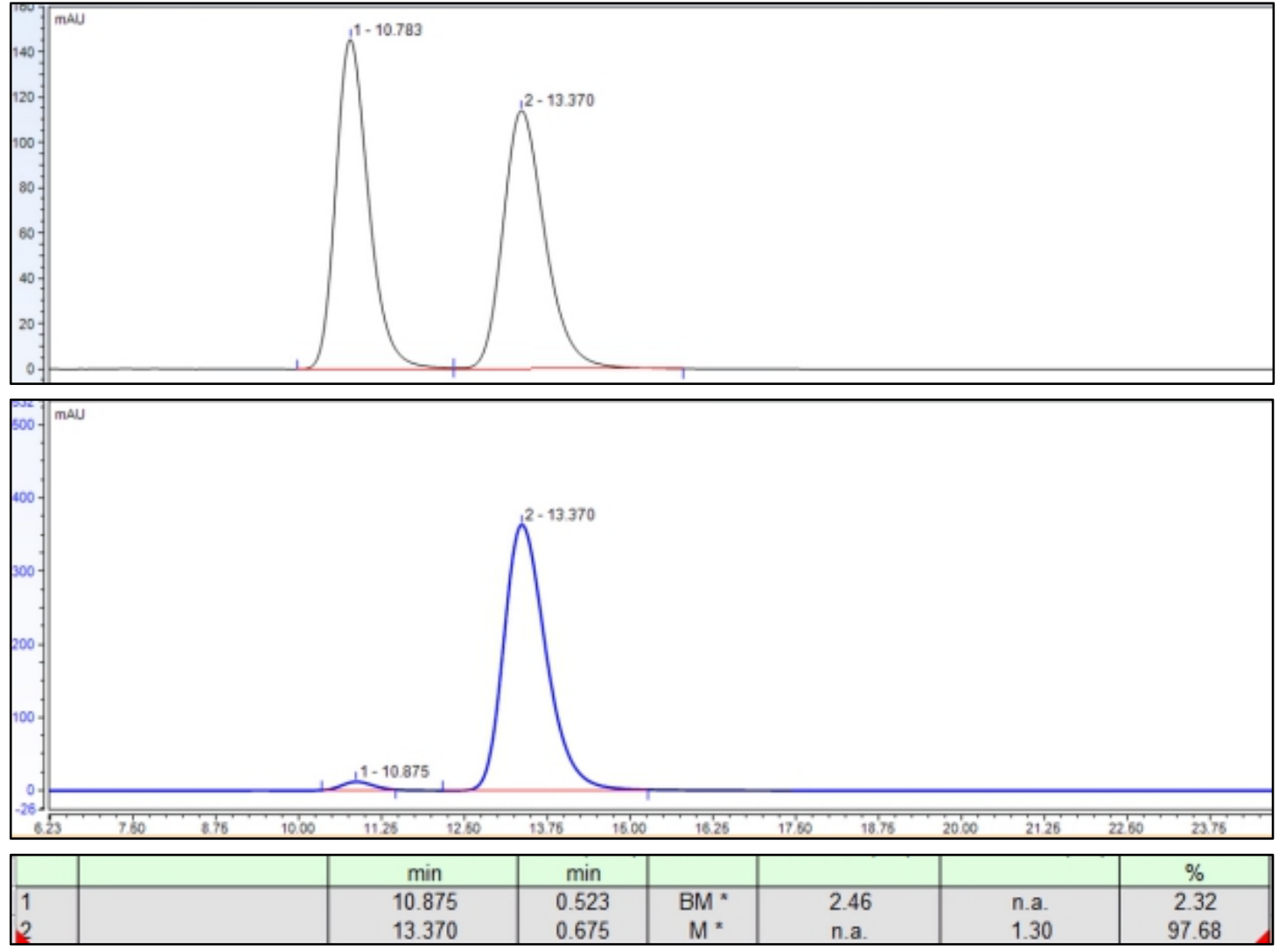

Figure S29. HPLC chromatogram of chiral product 2ac. 


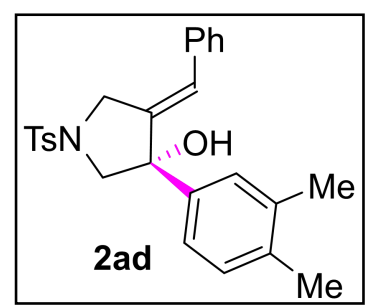

2ad: colorless oil, $(71.1 \mathrm{mg}, 82 \%),>99: 1$ er. $[\alpha]_{\mathrm{D}}{ }^{25}=-60.67(\mathrm{c}=1.0$, $\mathrm{CH}_{2} \mathrm{Cl}_{2}$ ); Enantiomeric ratio was determined by chiral HPLC. Chiralpark AD-H, $25{ }^{\circ} \mathrm{C}$, flow rate: $0.5 \mathrm{~mL} / \mathrm{min}$, hexanes/isopropanol: 15/85, $254 \mathrm{~nm}, 3.87 \mathrm{~min}(R), 4.87 \mathrm{~min}(S)$.

Figure S30. HPLC chromatogram of chiral product $\mathbf{2 a d .}$
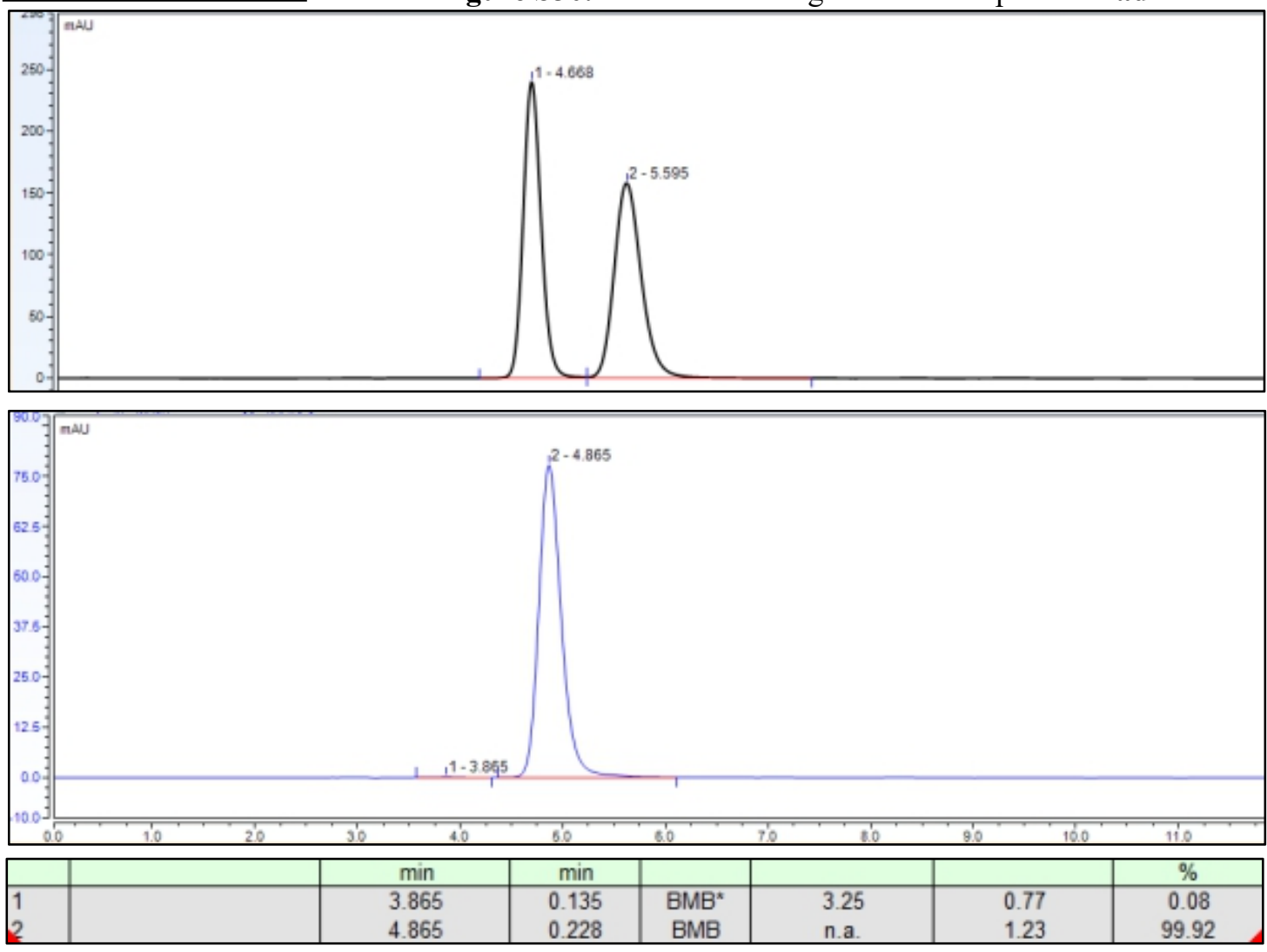


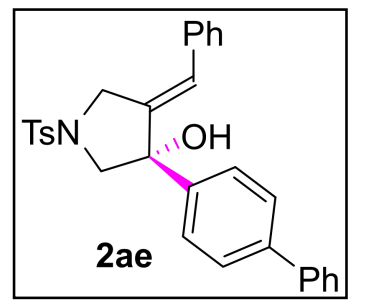

2ae: white solid, $(58.7 \mathrm{mg}, 61 \%), 99: 1$ er. $[\alpha]_{\mathrm{D}}{ }^{25}=-0.77\left(\mathrm{c}=1.0, \mathrm{CH}_{2} \mathrm{Cl}_{2}\right)$; Enantiomeric ratio was determined by chiral HPLC. Chiralpark AD-H, $25{ }^{\circ} \mathrm{C}$, flow rate: $0.5 \mathrm{~mL} / \mathrm{min}$, hexanes/isopropanol: 15/85, $254 \mathrm{~nm}, 9.56$ $\min (R), 11.36 \min (S)$.
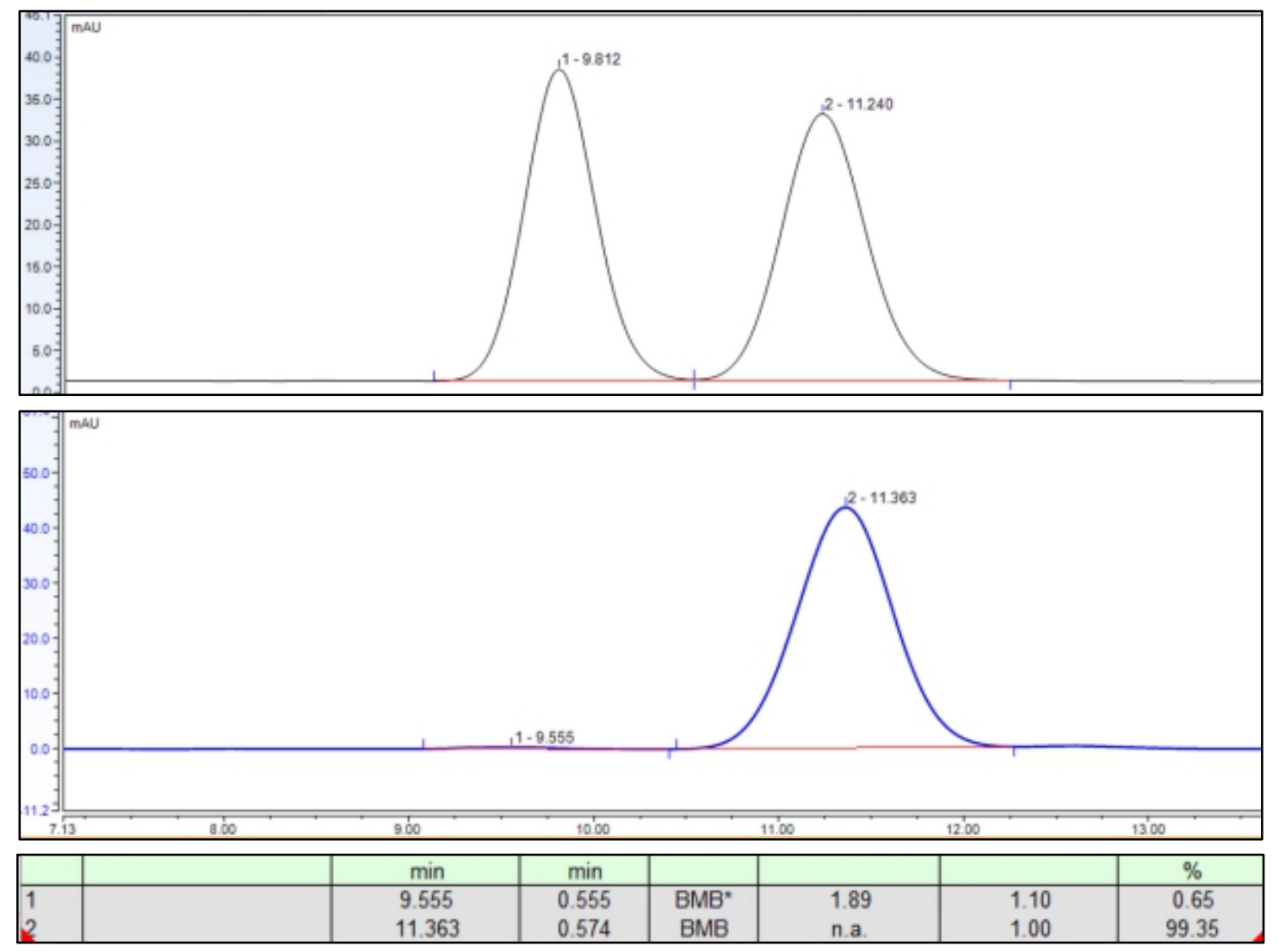

Figure S3I. HPLC chromatogram of chiral product 2 ae. 


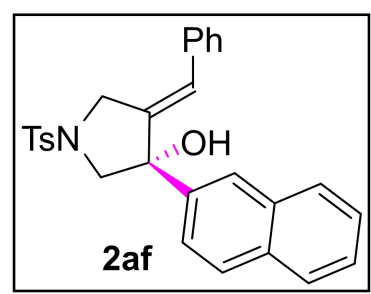

2af: white solid, $(79.1 \mathrm{mg}, 87 \%), 99: 1$ er. $[\alpha]_{\mathrm{D}}{ }^{25}=-43.36$ (c $=$ 1.0, $\mathrm{CH}_{2} \mathrm{Cl}_{2}$ ); Enantiomeric ratio was determined by chiral HPLC. Chiralpark AD-H, $25{ }^{\circ} \mathrm{C}$, flow rate: $0.5 \mathrm{~mL} / \mathrm{min}$, hexanes/isopropanol: $15 / 85,254 \mathrm{~nm}, 8.44 \mathrm{~min}(S), 19.25 \min (R)$.
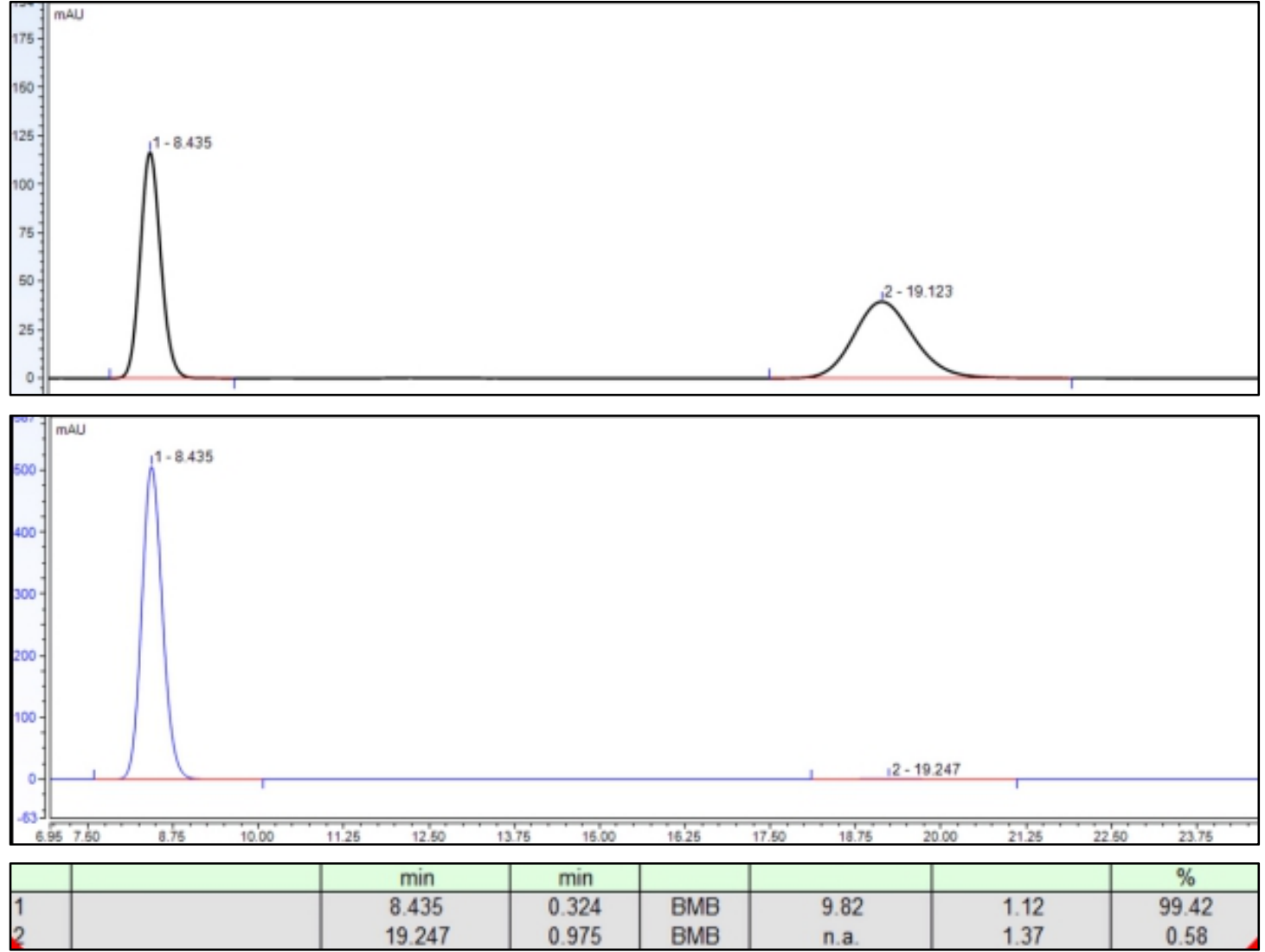

Figure S32. HPLC chromatogram of chiral product 2 af. 


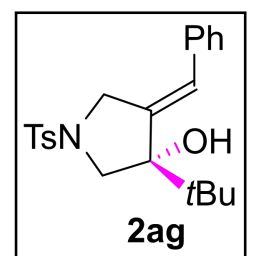

2ag: white solid, $(60.9 \mathrm{mg}, 79 \%), 98: 2$ er. $[\alpha]_{\mathrm{D}}^{25}=+7.2\left(\mathrm{c}=1.0, \mathrm{CH}_{2} \mathrm{Cl}_{2}\right)$; Enantiomeric ratio was determined by chiral HPLC. Chiralpark AD-H, $25{ }^{\circ} \mathrm{C}$, flow rate: $0.5 \mathrm{~mL} / \mathrm{min}$, hexanes/isopropanol: $15 / 85,254 \mathrm{~nm}, 5.50 \mathrm{~min}(S), 5.95$ $\min (R)$.
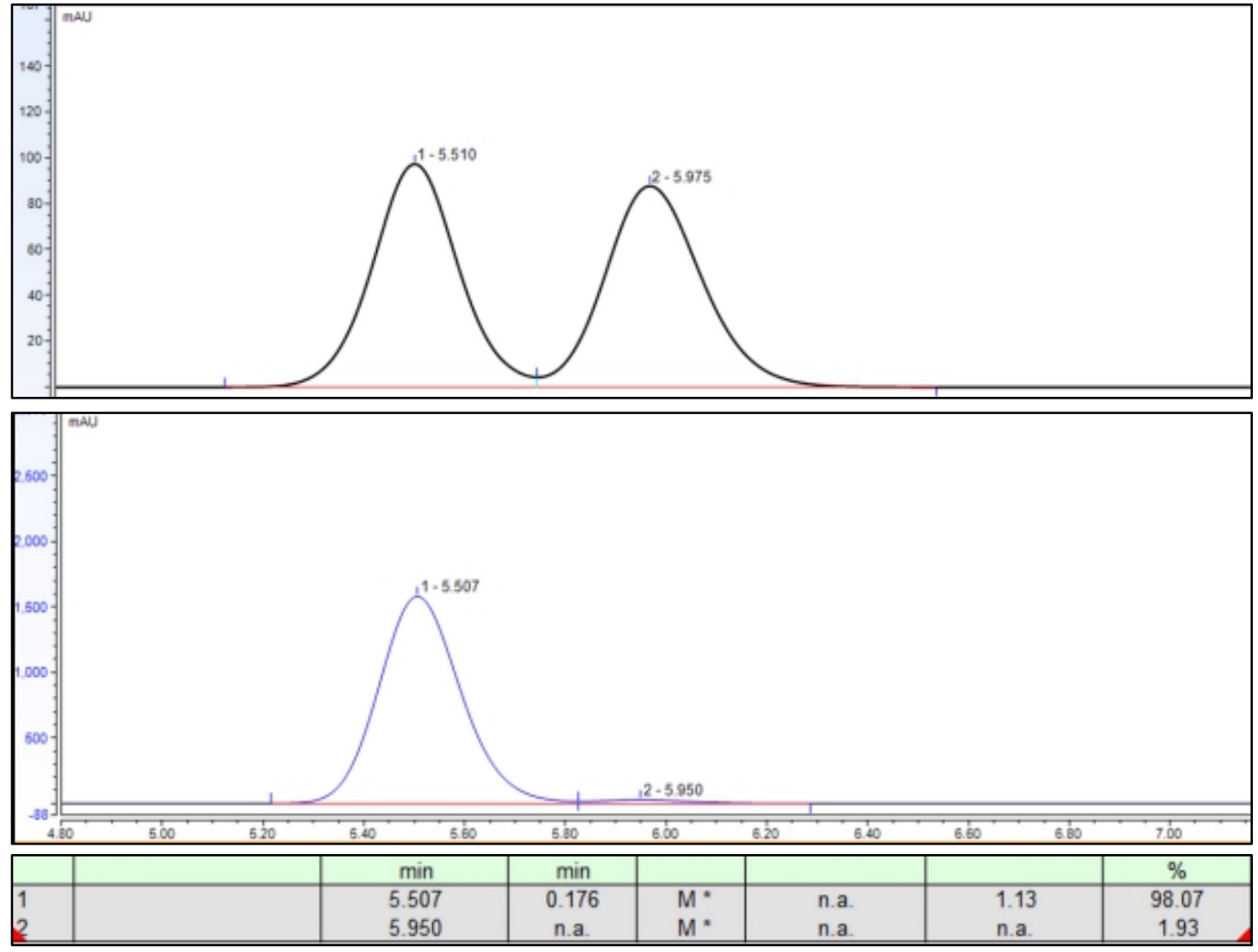

Figure S33. HPLC chromatogram of chiral product 2ag. 


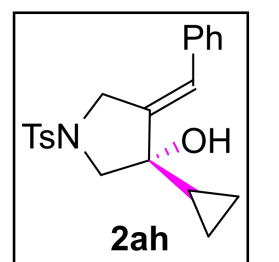

2ah: colorless oil, (59.8mg, 81\%), 99:1 er. $[\alpha]_{\mathrm{D}}{ }^{25}=-12.0\left(\mathrm{c}=1.0, \mathrm{CH}_{2} \mathrm{Cl}_{2}\right)$; Enantiomeric ratio was determined by chiral HPLC. Chiralpark AD-H, $25{ }^{\circ} \mathrm{C}$, flow rate: $0.5 \mathrm{~mL} / \mathrm{min}$, hexanes/isopropanol: 20/80, $254 \mathrm{~nm}, 9.56 \mathrm{~min}(S), 12.06$ $\min (R)$.
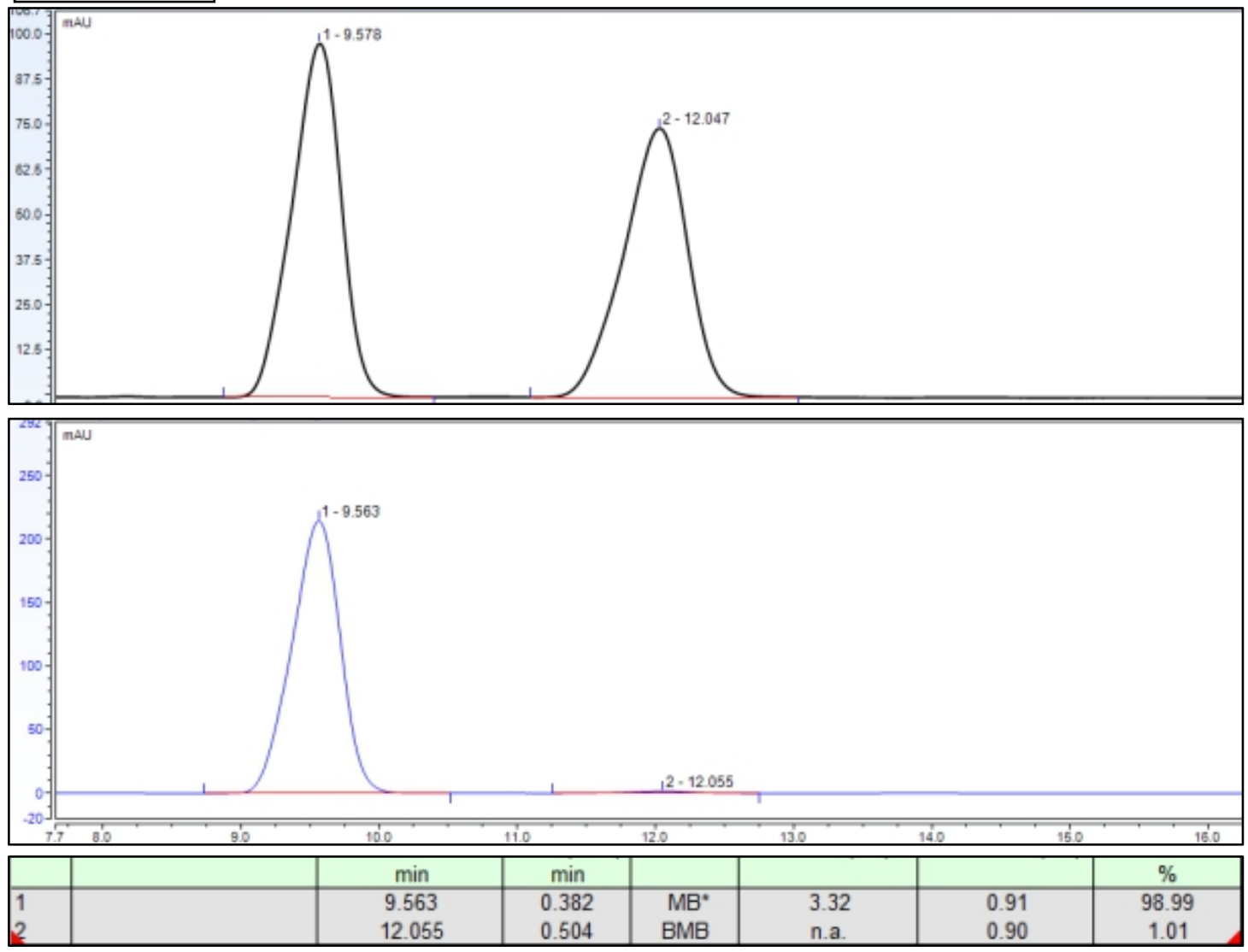

Figure S34. HPLC chromatogram of chiral product 2ah. 


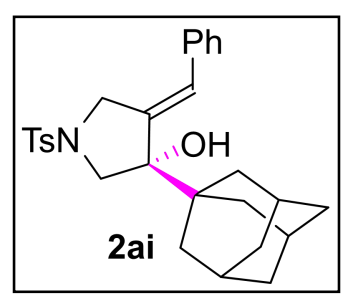

2ai: white solid, (70.4mg, 76\%), 99:1 er, $[\alpha]_{\mathrm{D}}^{25}=-11.2\left(\mathrm{c}=1.0, \mathrm{CH}_{2} \mathrm{Cl}_{2}\right)$; Enantiomeric ratio was determined by chiral HPLC. Chiralpark AD-H, 25 ${ }^{\circ} \mathrm{C}$, flow rate: $0.5 \mathrm{~mL} / \mathrm{min}$, hexanes/isopropanol: $20 / 80,254 \mathrm{~nm}, 6.26 \mathrm{~min}$ $(R), 6.85 \min (S)$.
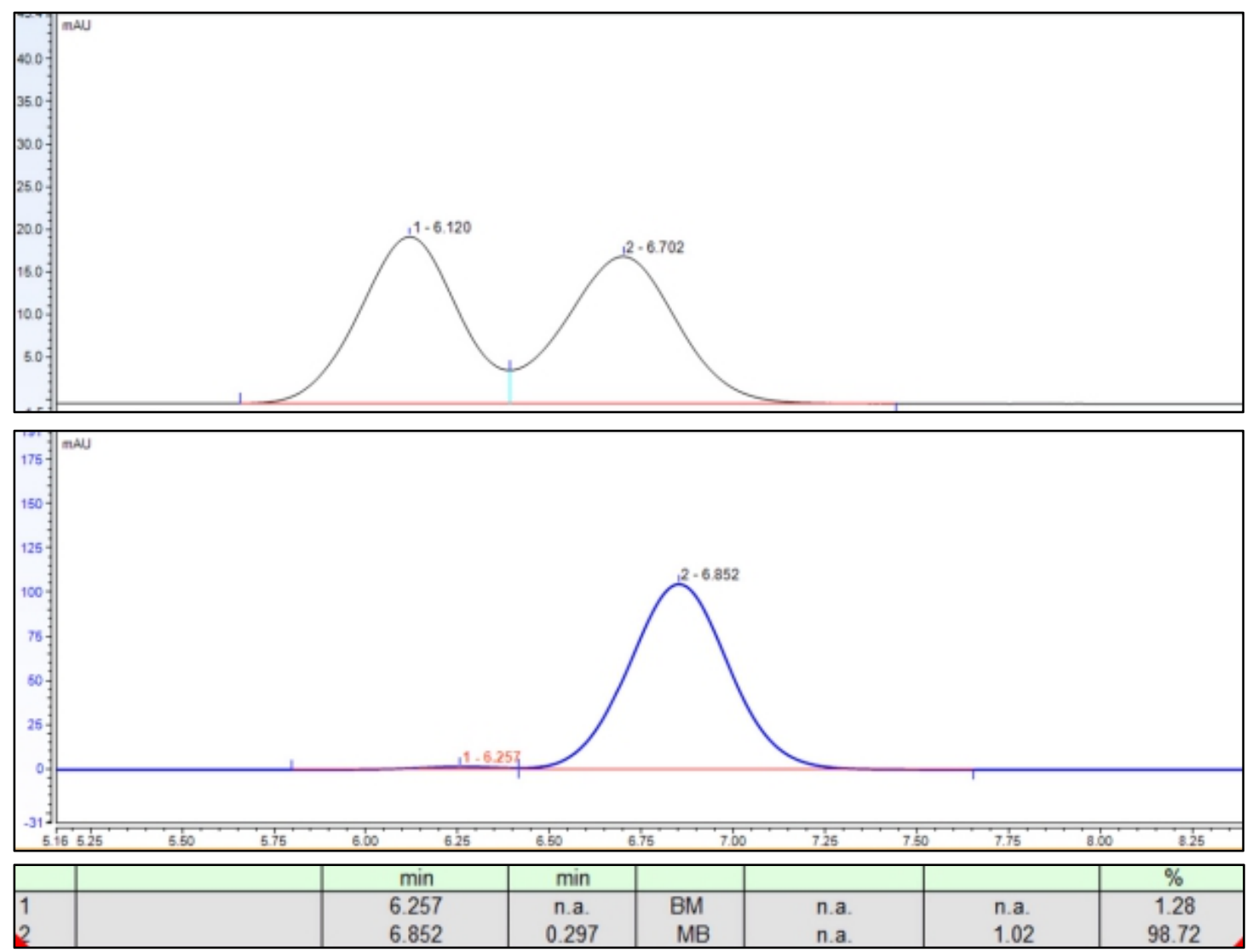

Figure S35. HPLC chromatogram of chiral product 2ai. 


\section{Discovered new asymmetric couplings with $(R)$-AntPhos and $N$-1,6-alkynone}

a)

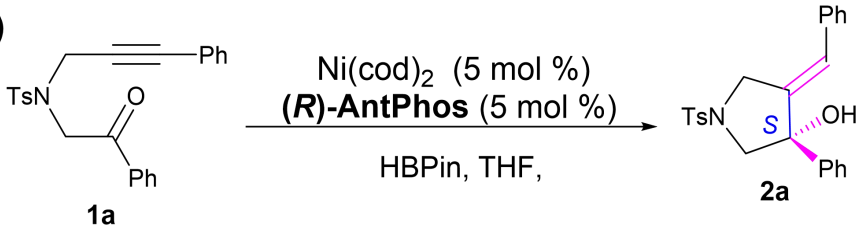

Scheme S2. Discover new asymmetric coupling reaction(a).

Synthetic procedure: In a glove box, add $\mathrm{Ni}(\mathrm{COD})_{2}(2.8 \mathrm{mg}, 0.010 \mathrm{mmol}, 5 \mathrm{~mol} \%)$, $(\boldsymbol{R})$-Antphos $(3.7 \mathrm{mg}, 0.010 \mathrm{mmol}, 5 \mathrm{~mol} \%)$ and dioxane $(0.5 \mathrm{~mL})$ were added to a $5 \mathrm{~mL}$ screw-cap vial equipped with a magnetic stirring bar. Substrate 1a $(83.4 \mathrm{mg}, 0.20 \mathrm{mmol}, 1.00$ equiv) and Pinacolborane (HBPin, 73.1, $0.60 \mathrm{mmol}, 3.00$ equiv) was added to the solution in one portion. The vial was capped with a screw cap, and the resulting mixture was stirred at $70{ }^{\circ} \mathrm{C}$ for $24 \mathrm{~h}$, quenched with saturated $\mathrm{NaHCO}_{3}$ solution, extracted with EtOAc, washed with saturated brine, dried over anhydrous sodium sulfate, filtered, and concentrated under vacuum. The residue was purified by column chromatography to obtain compound $\mathbf{2 a}$.

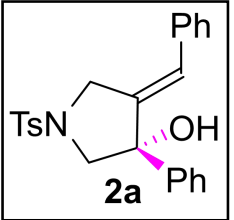

2a: white solid, $(71.4 \mathrm{mg}, 88 \%) 81: 19$ er. $[\alpha]_{\mathrm{D}}{ }^{25}=-22.0\left(\mathrm{c}=1.0, \mathrm{CH}_{2} \mathrm{Cl}_{2}\right)$; Enantiomeric ratio was determined by chiral HPLC. Chiralpark AD-H, $25{ }^{\circ} \mathrm{C}$, flow rate: $0.5 \mathrm{~mL} / \mathrm{min}$, hexanes/isopropanol: $15 / 85,254 \mathrm{~nm}, 12.58 \mathrm{~min}(S)$, $15.07 \mathrm{~min}(R)$. All other physical and spectroscopic data of this compound were identical to those mentioned above for compound $\mathbf{2 a}$.
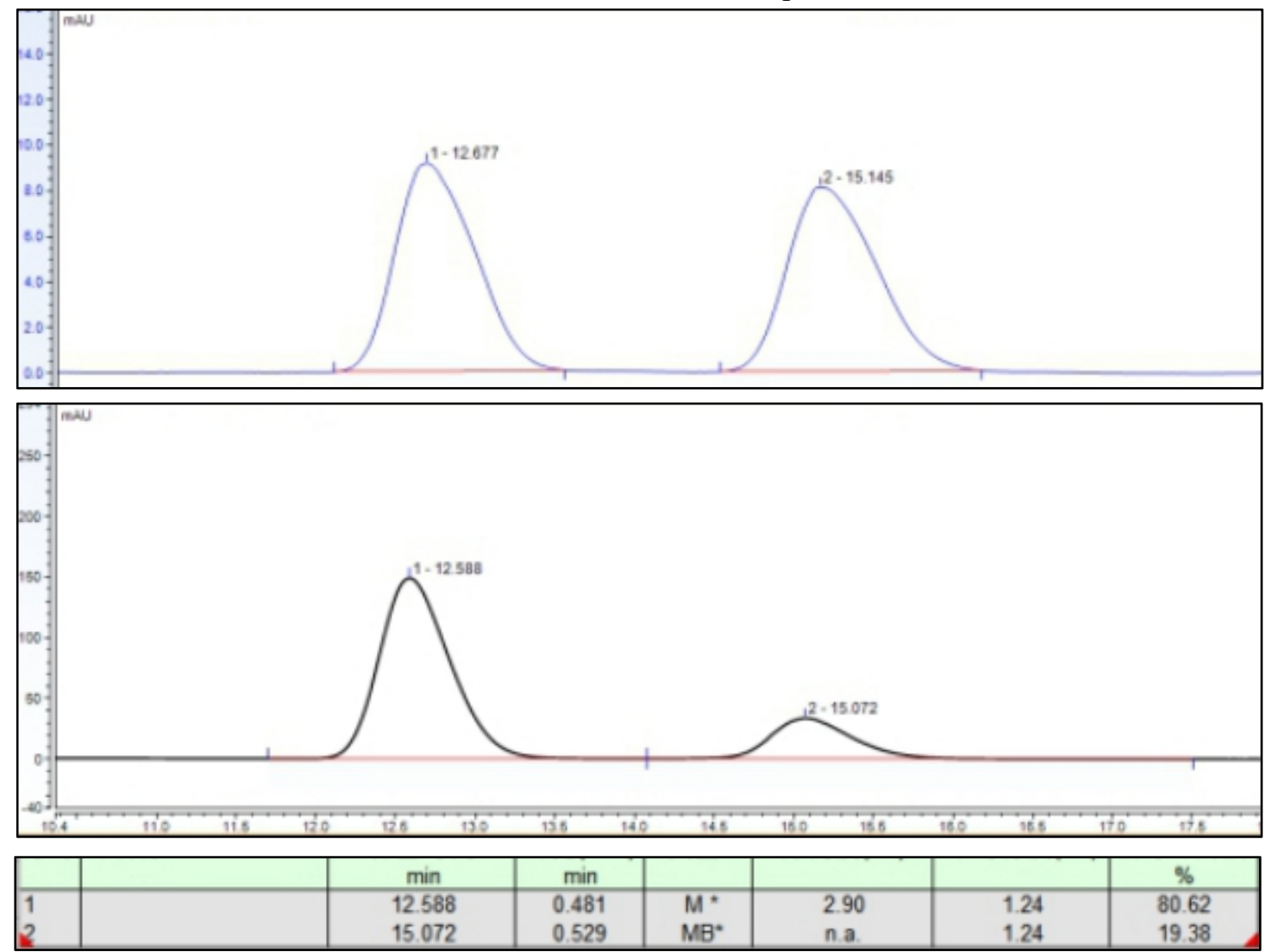

Figure S36. HPLC chromatogram of chiral product 2a. 
b)

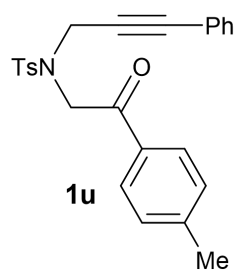

$\mathrm{Ni}(\mathrm{Cod})_{2}(5 \mathrm{OH})_{2}$ mol \%)

(R)-AntPhos (6 mol \%)

Dioxane/tBuOH

$(5 / 1), 70^{\circ} \mathrm{C}$

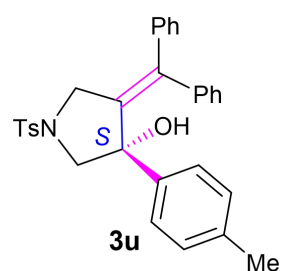

Scheme S3. Discover new asymmetric coupling reaction(b).

Synthetic procedure: In the glove box, add $\mathrm{Ni}(\mathrm{COD})_{2}(2.8 \mathrm{mg}, 0.010 \mathrm{mmol}, 5 \mathrm{~mol} \%)$, $(\boldsymbol{R})$-Antphos $(3.7 \mathrm{mg}, 0.010 \mathrm{mmol}, 5 \mathrm{~mol} \%)$ and dioxane $(0.5 \mathrm{~mL})$ were added to a $5 \mathrm{~mL}$ screw-cap vial equipped with a magnetic stirring bar. Substrate 1u $(83.4 \mathrm{mg} 0.20 \mathrm{mmol}, 1.00$ equiv), Phenylboronic Acid $\left[\mathrm{PhB}(\mathrm{OH})_{2}, 73.1 \mathrm{mg}, 0.60 \mathrm{mmol}, 3.00\right.$ equiv] and tert-butanol $(t \mathrm{BuOH}$, $0.1 \mathrm{ml}$ ) was added to the solution in one portion. The vial was capped with a screw cap, and the resulting mixture was stirred at $70{ }^{\circ} \mathrm{C}$ for $24 \mathrm{~h}$, quenched with saturated $\mathrm{NaHCO}_{3}$ solution, extracted with EtOAc, washed with saturated brine, dried over anhydrous sodium sulfate, filtered, and concentrated under vacuum. The residue was purified by column chromatography to obtain compound $\mathbf{3 u}$.

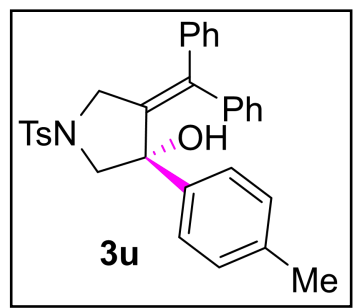

3u: colorless oil, $(83.2 \mathrm{mg}, 82 \%), 93: 7$ er. $[\alpha]_{\mathrm{D}}{ }^{25}=-12.12(\mathrm{c}=1.0$, $\mathrm{CH}_{2} \mathrm{Cl}_{2}$ ); Enantiomeric ratio was determined by chiral HPLC. Chiralpark $\mathrm{AD}-\mathrm{H}, 25{ }^{\circ} \mathrm{C}$, flow rate: $0.5 \mathrm{~mL} / \mathrm{min}$, hexanes/isopropanol: $15 / 85,254$ $\mathrm{nm}, 8.88 \min (S), 17.36 \min (R)$.
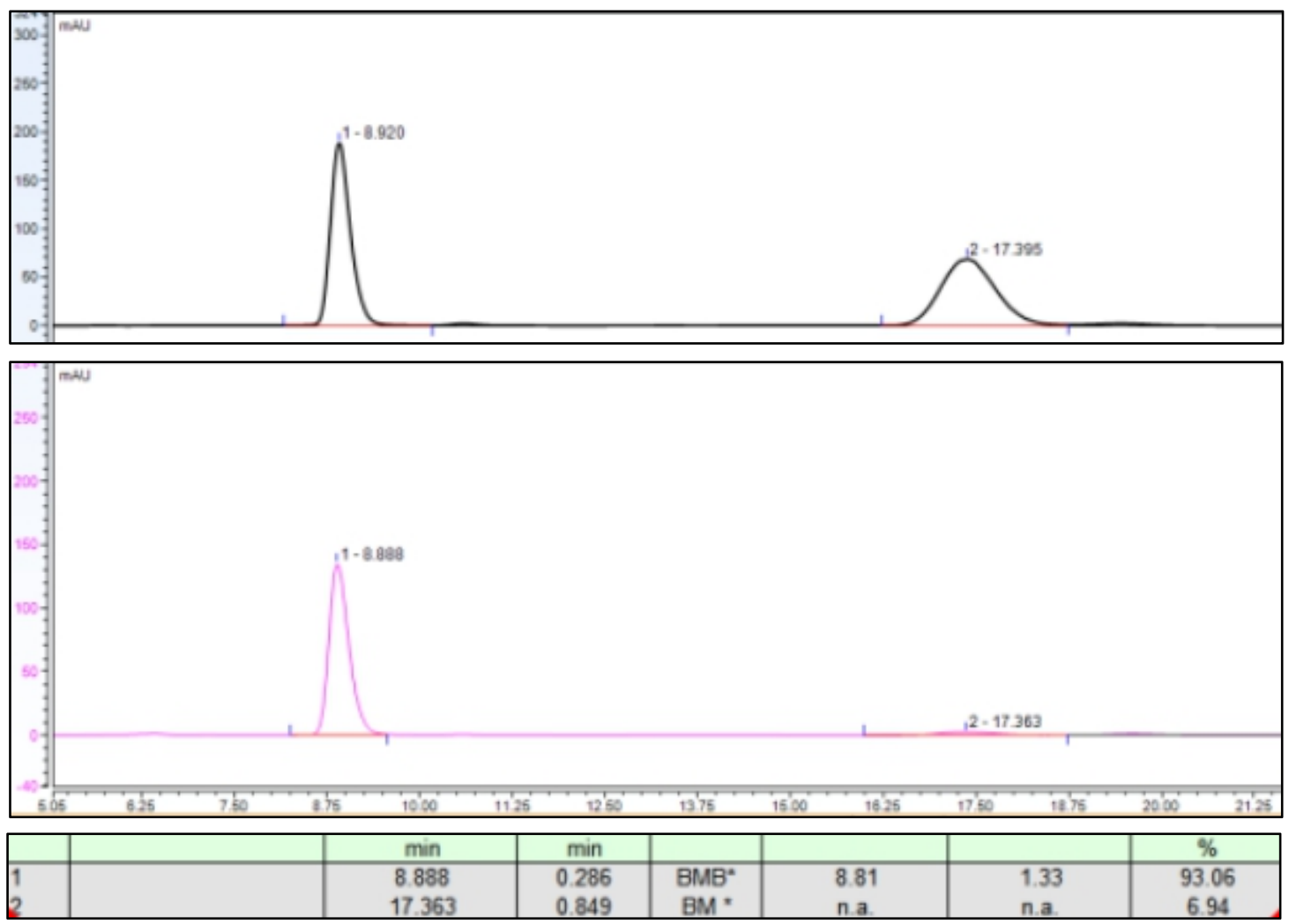

Figure S37. HPLC chromatogram of chiral product $3 \mathbf{u}$. 
4. NMR Spectra

음

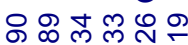

NNNN

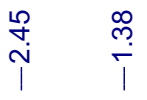

Ts $-N^{H}$

S2 Boc

${ }^{1} \mathrm{H}$ NMR (500 MHz, $\mathrm{CDCl}_{3}$ )
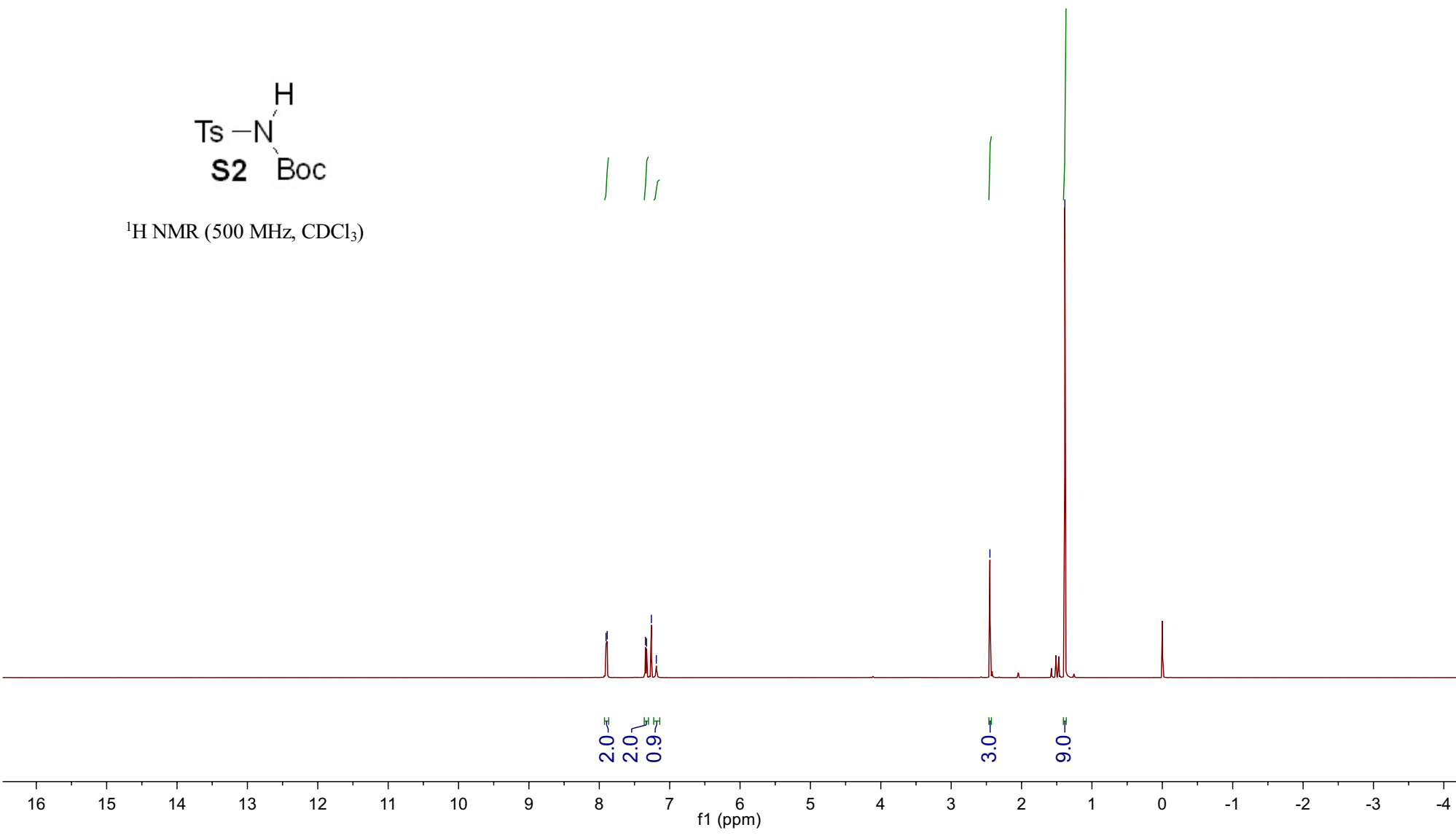
은

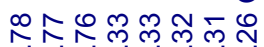

NNNNNNN

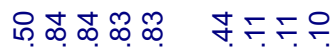

サn்
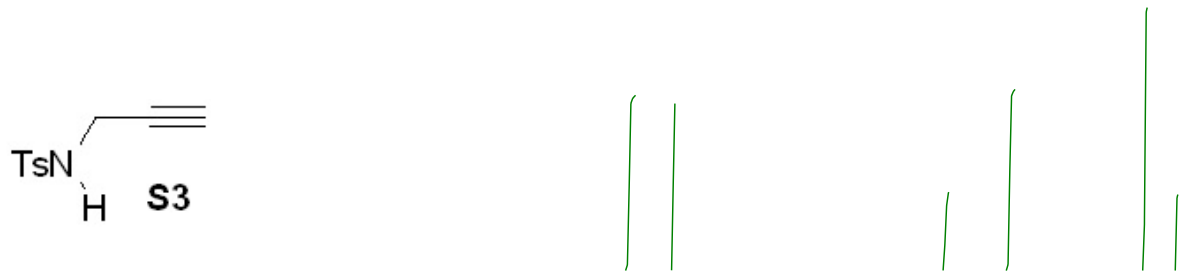

${ }^{1} \mathrm{H}$ NMR (500 MHz, $\mathrm{CDCl}_{3}$ )

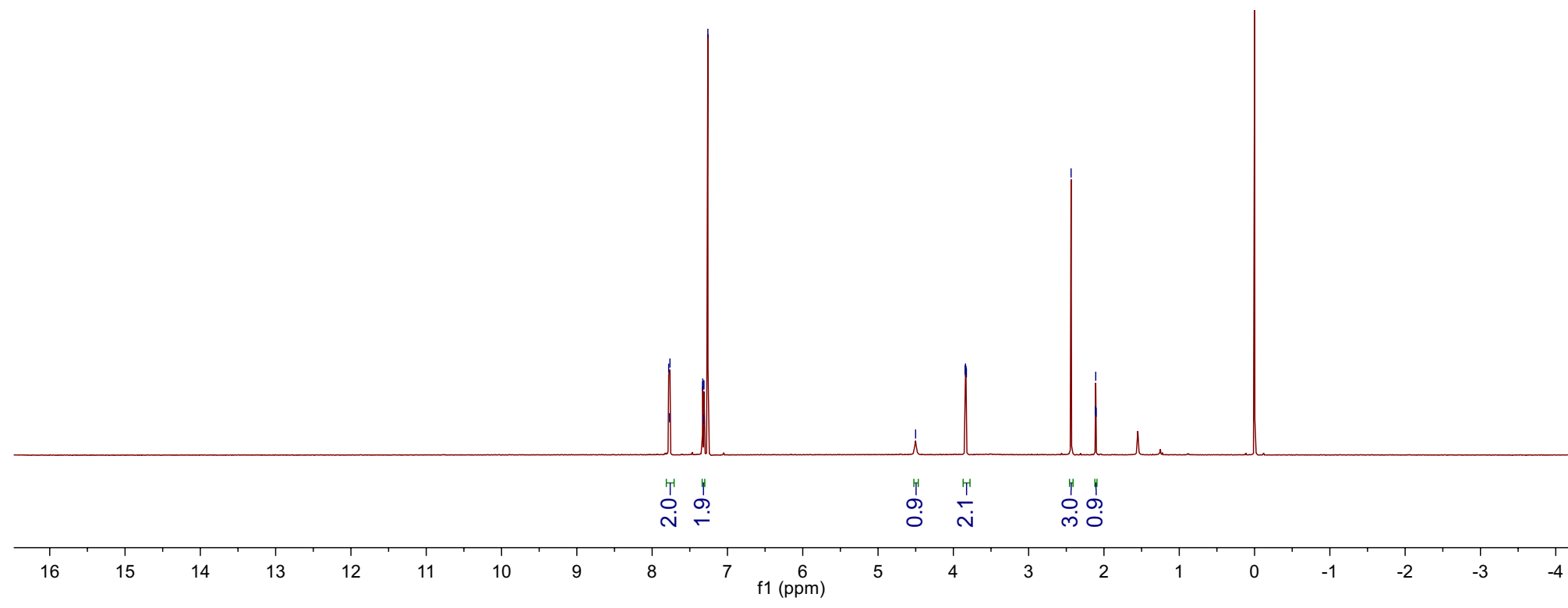


㠃

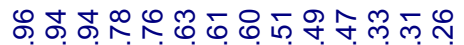

ríñNáñNán

$\ltimes \stackrel{\infty}{N} \stackrel{\infty}{\sim}$

곤

กู่
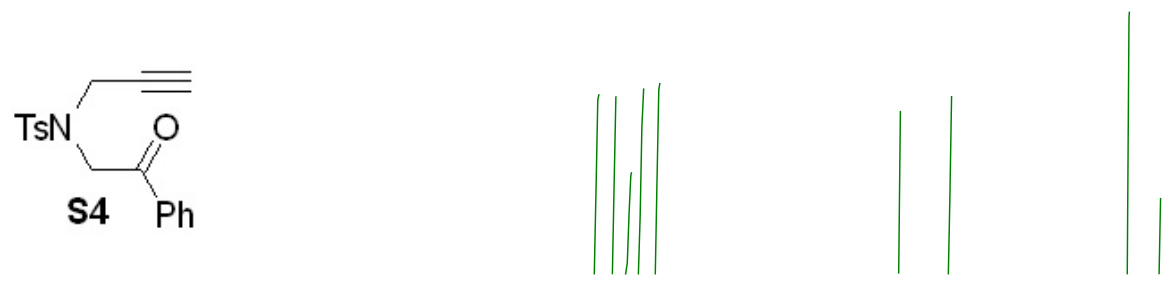

${ }^{1} \mathrm{H}$ NMR (500 MHz, $\mathrm{CDCl}_{3}$ )

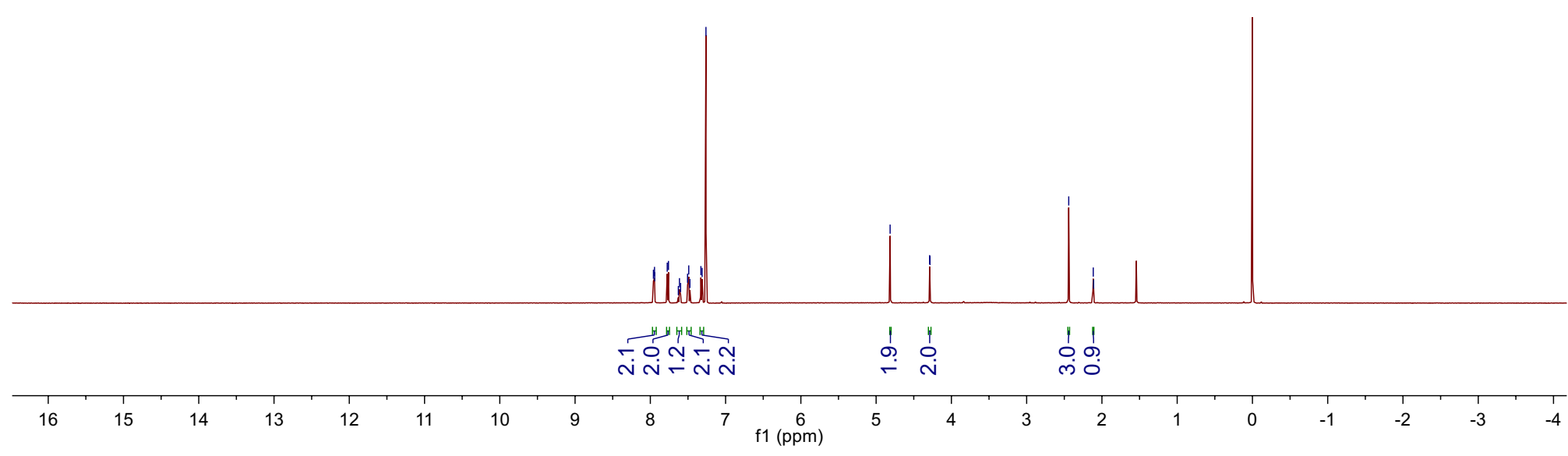




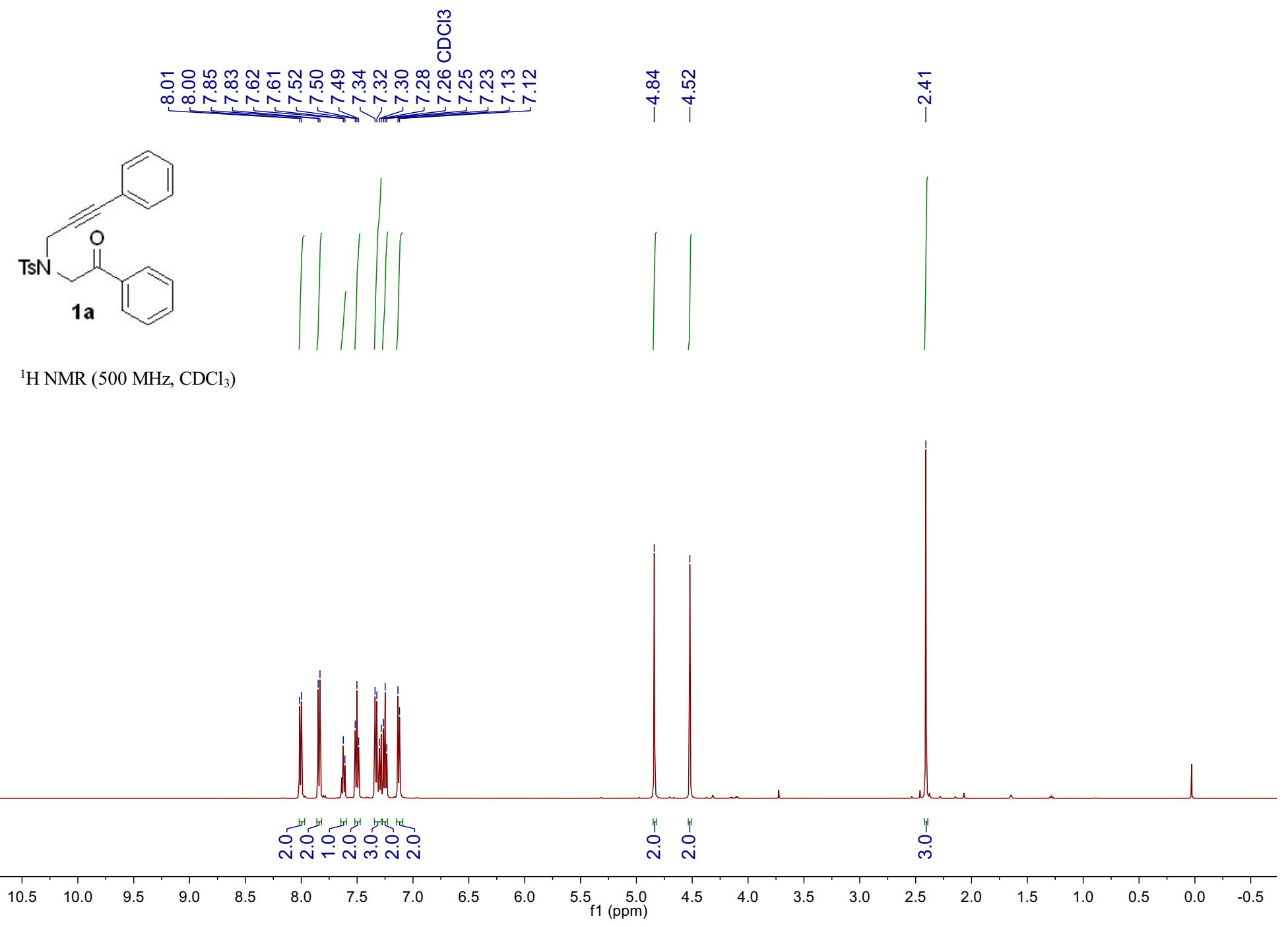



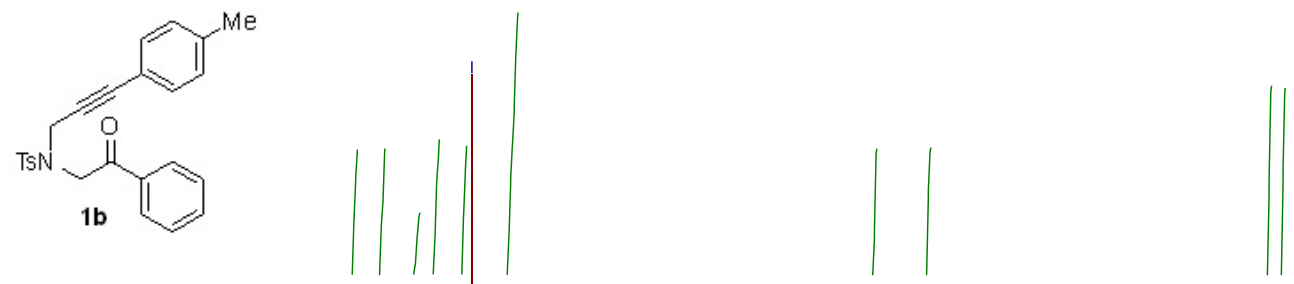

${ }^{1} \mathrm{H}$ NMR (500 MHz, $\mathrm{CDCl}_{3}$ )

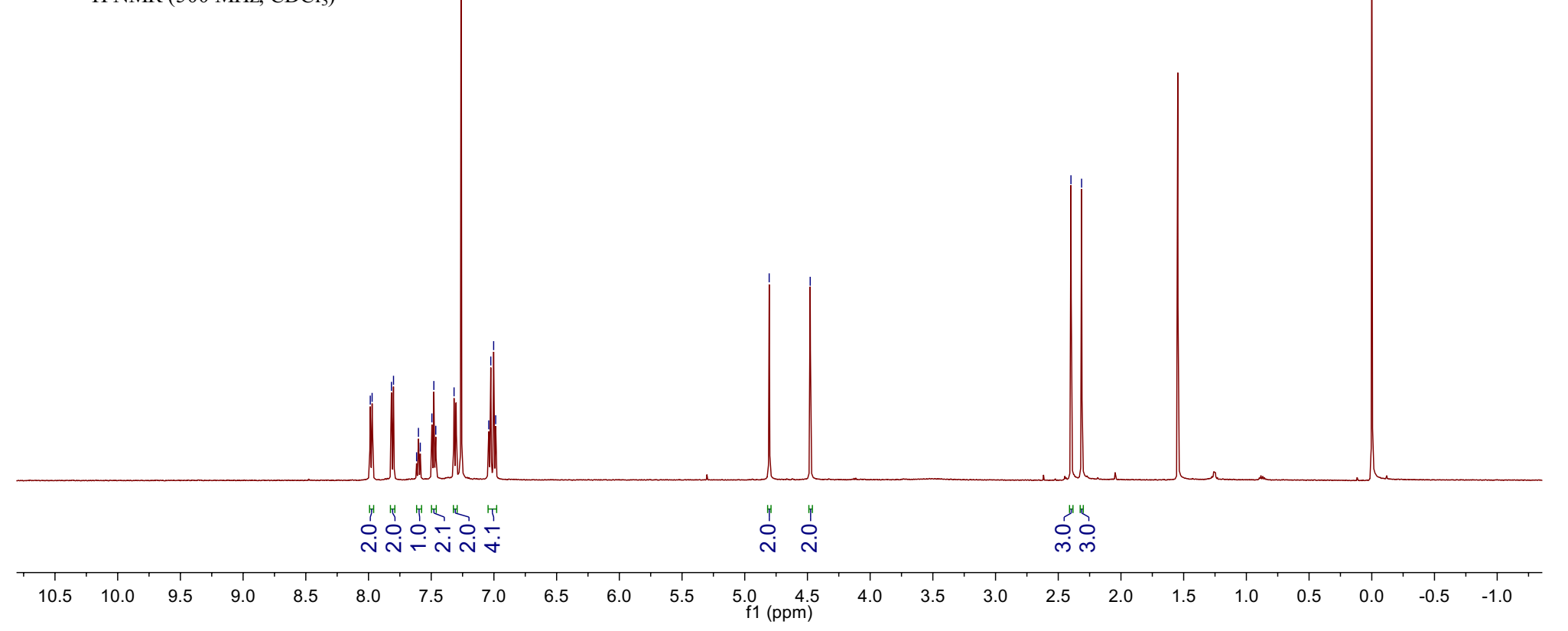




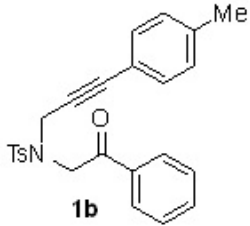

${ }^{13} \mathrm{C}$ NMR $\left(126 \mathrm{MHz}, \mathrm{CDCl}_{3}\right)$

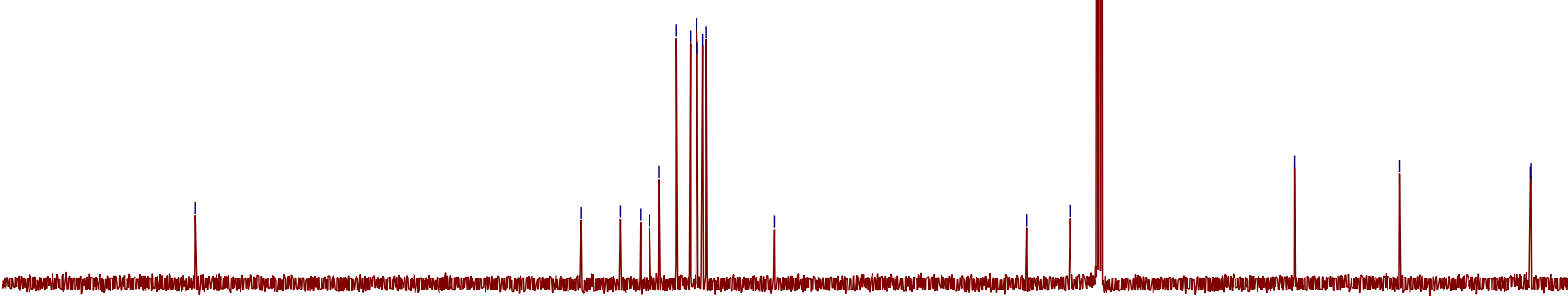

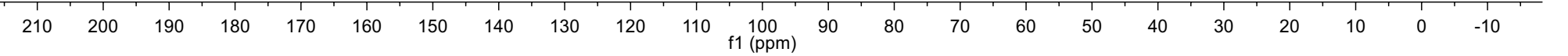


రె 60009

ขูก ก ก

กักฺฺุ

ז广
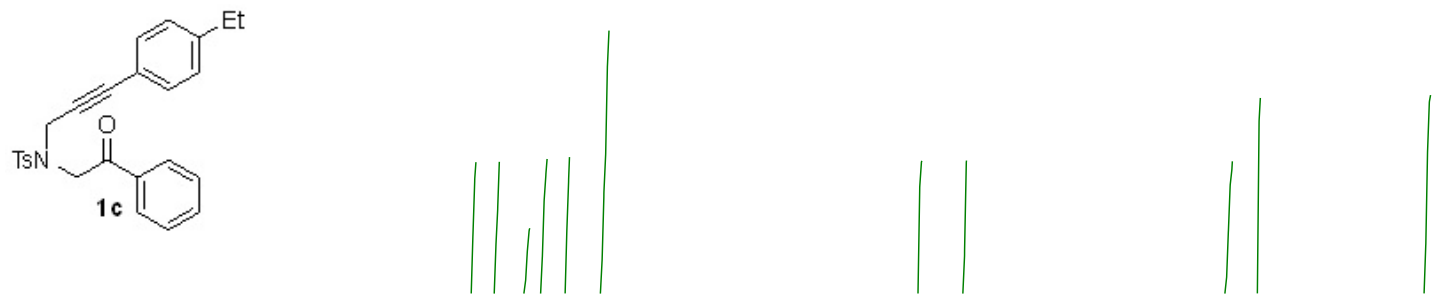

${ }^{1} \mathrm{H} \mathrm{NMR}\left(500 \mathrm{MHz}, \mathrm{CDCl}_{3}\right.$ )

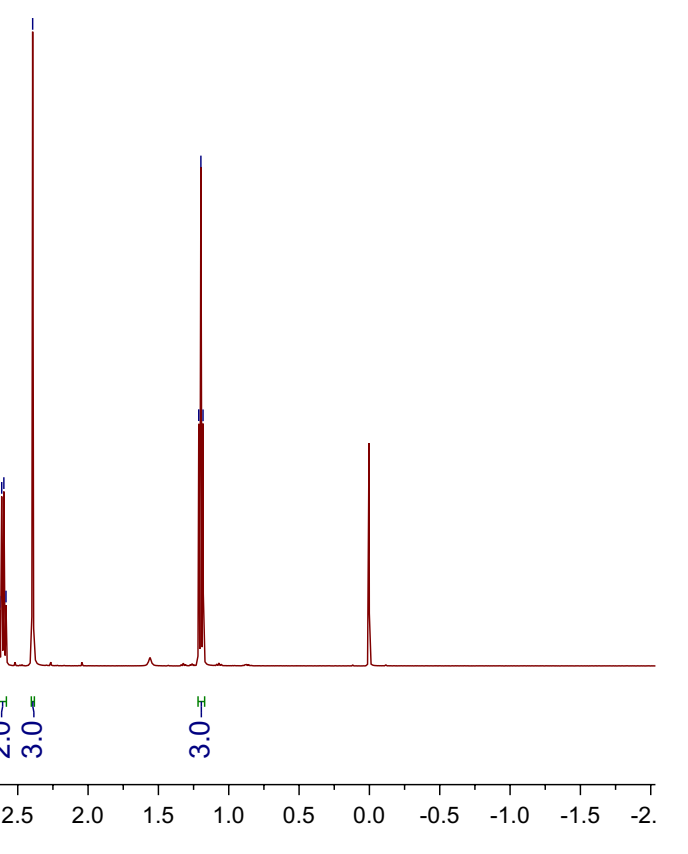


iุ?

๓ัฐ

i

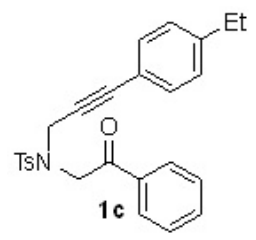

${ }^{13} \mathrm{C}$ NMR $\left(126 \mathrm{MHz}, \mathrm{CDCl}_{3}\right)$

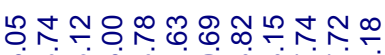

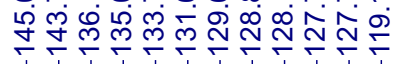

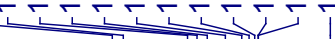

$m \frac{m}{0}$

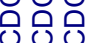

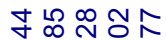

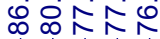

$\longrightarrow$ $\begin{array}{lll}\hat{\infty} & \hat{m} & \hat{N} N \tilde{N} \\ \overline{1} & \infty & \infty\end{array}$

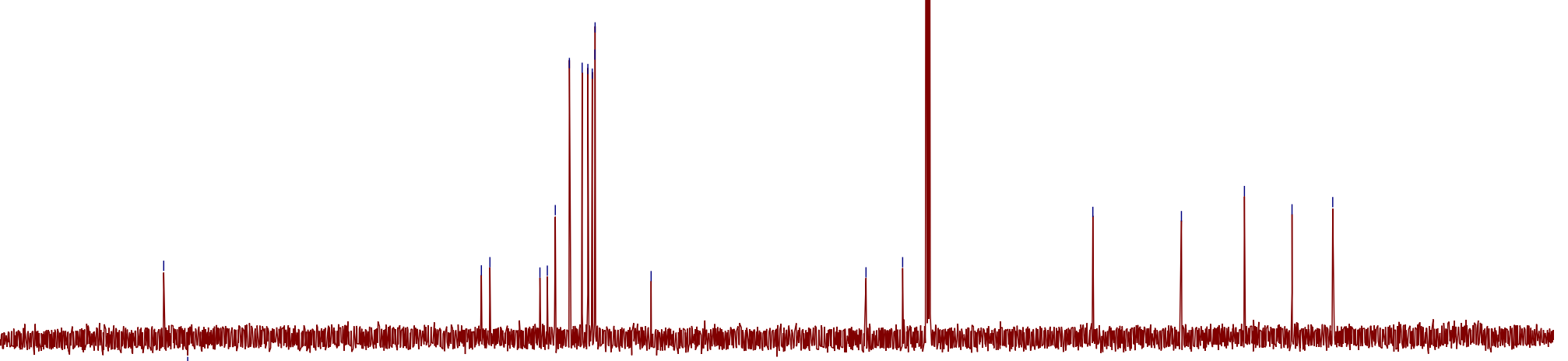


鱼

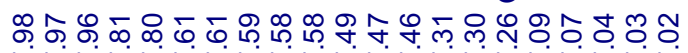

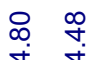

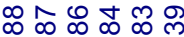

సัก

NNNNNNNNNNNNNNN
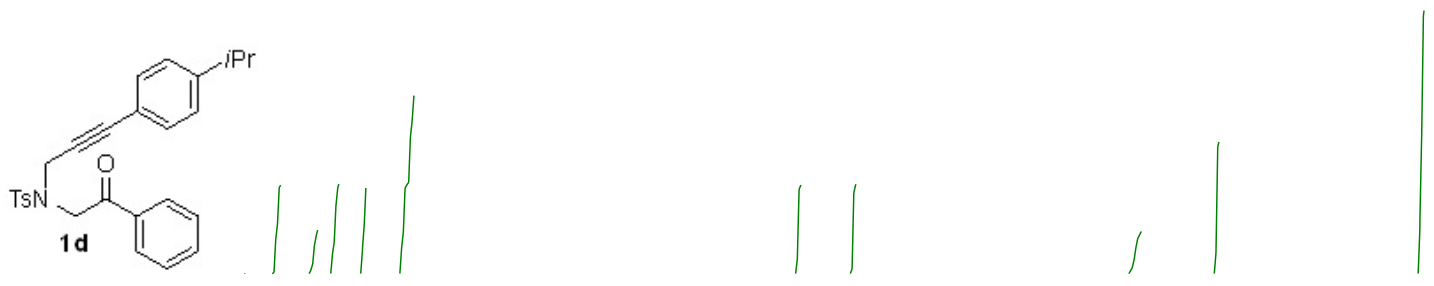

${ }^{1} \mathrm{H} \mathrm{NMR}\left(500 \mathrm{MHz}, \mathrm{CDCl}_{3}\right)$

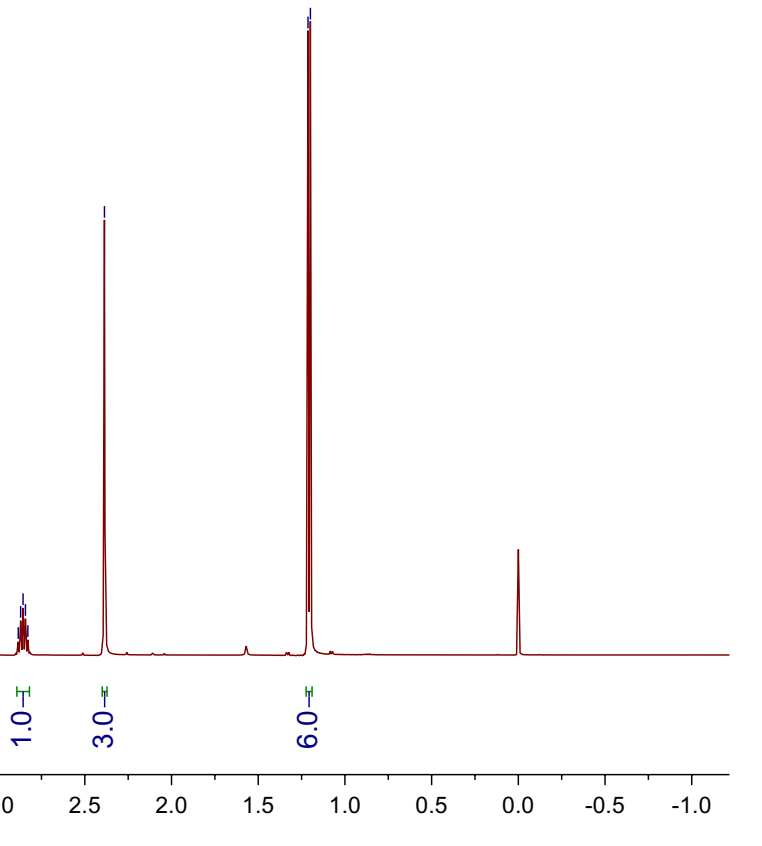




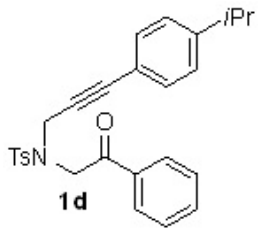

${ }^{13} \mathrm{C} \mathrm{NMR}\left(126 \mathrm{MHz}, \mathrm{CDCl}_{3}\right)$

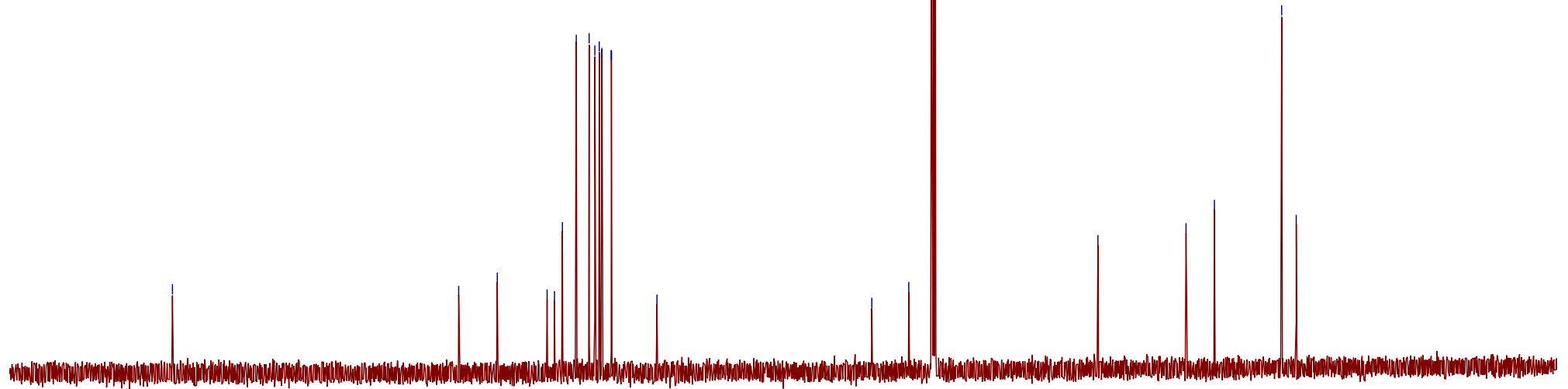

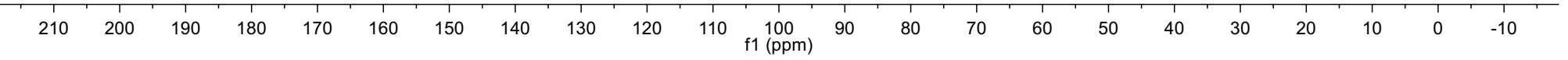


บํำ

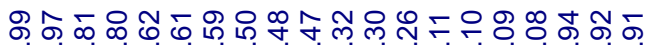

N
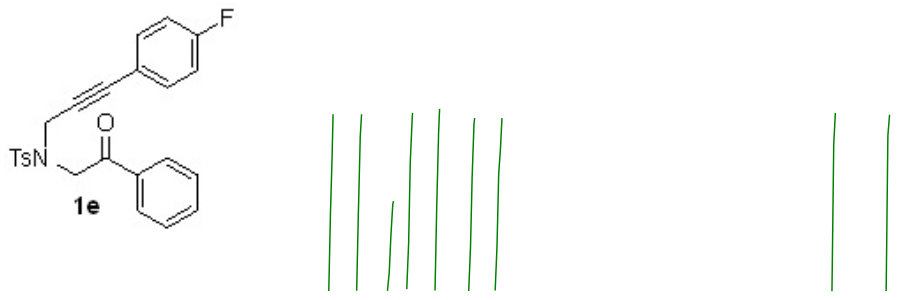

${ }^{1} \mathrm{H} \mathrm{NMR}\left(500 \mathrm{MHz}, \mathrm{CDCl}_{3}\right)$

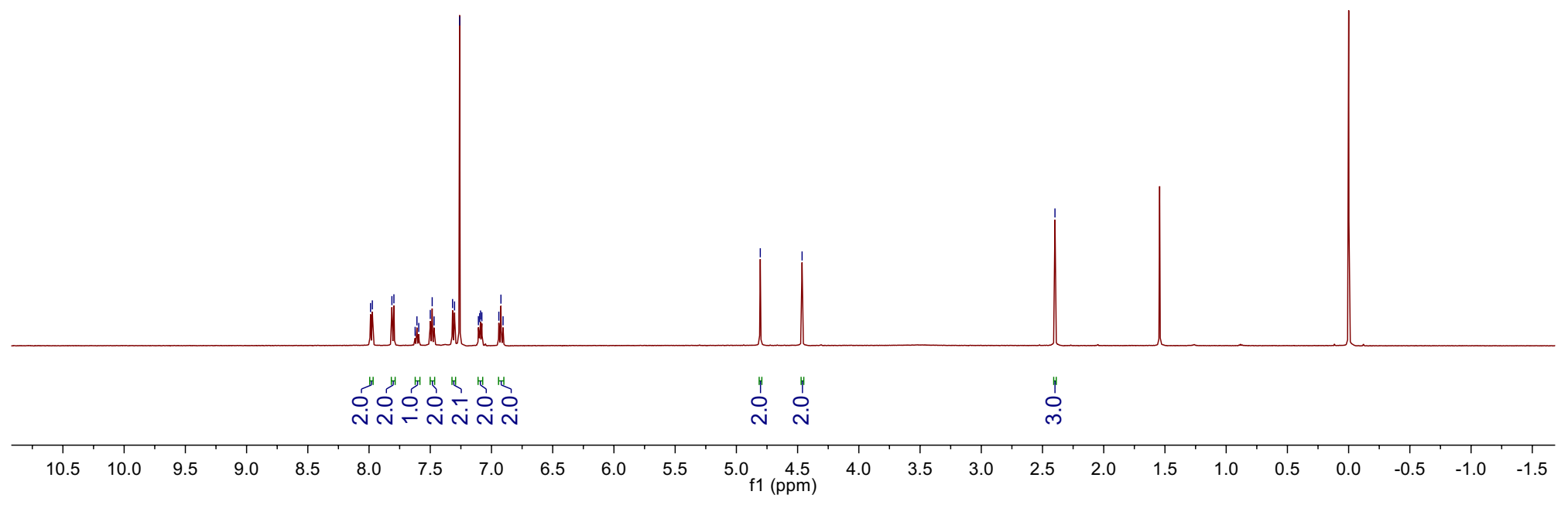




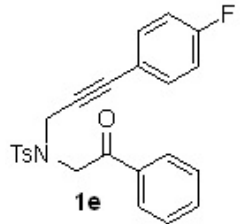

${ }^{13} \mathrm{C} \mathrm{NMR}\left(126 \mathrm{MHz}, \mathrm{CDCl}_{3}\right)$

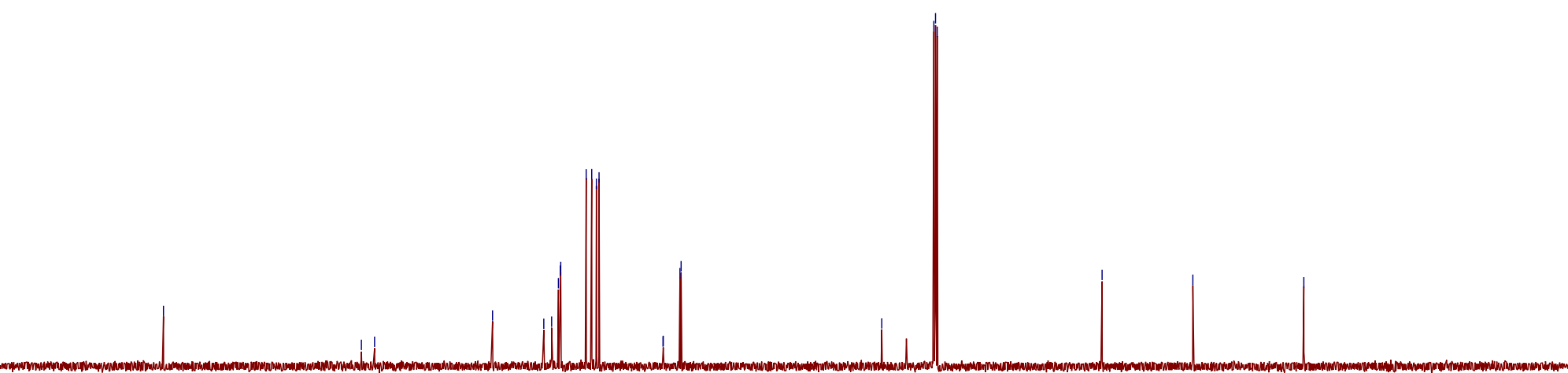

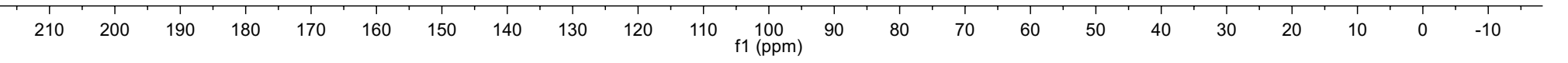




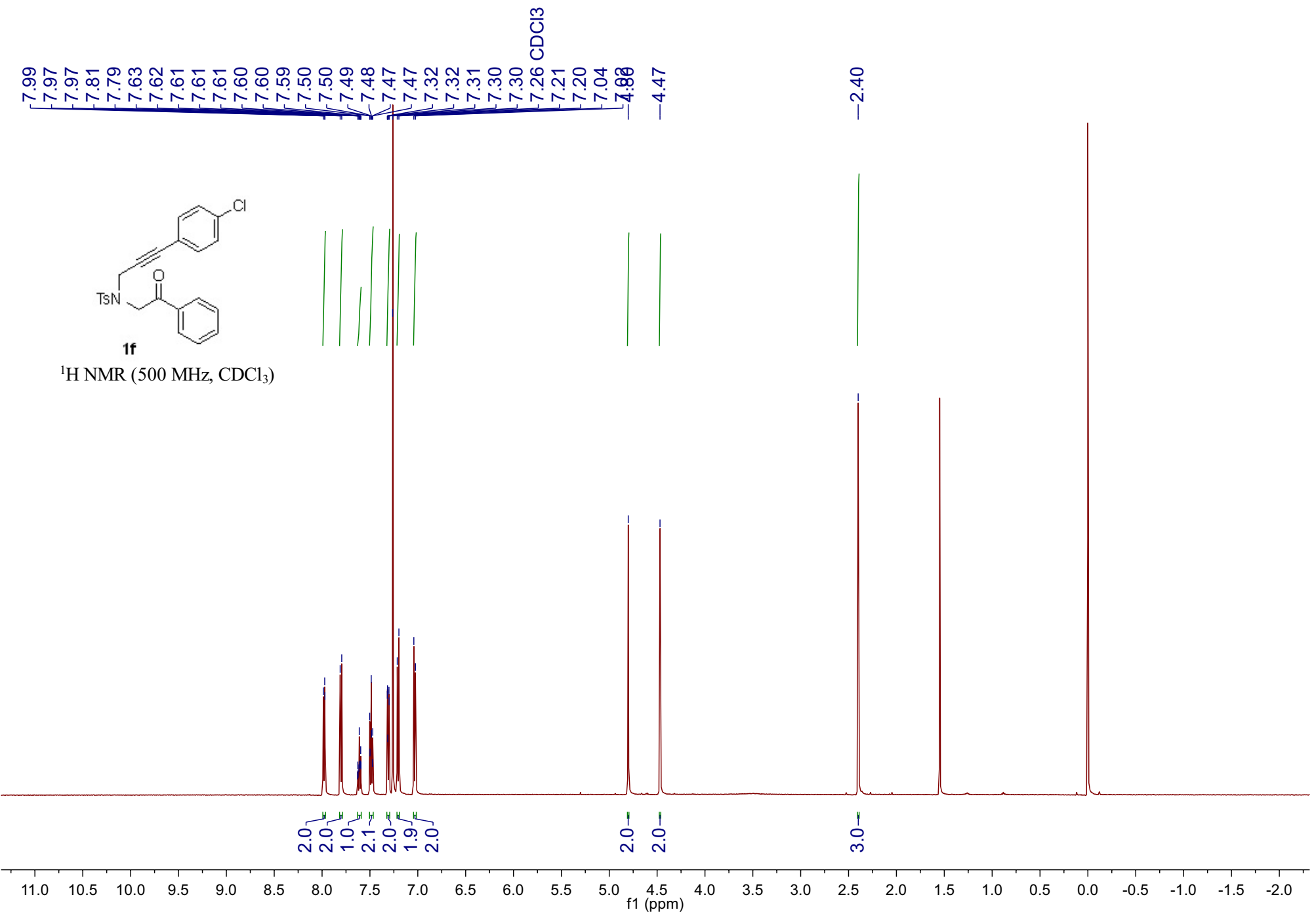




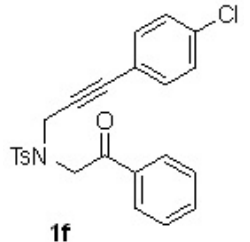

1)$$
1 f
$$

${ }^{13} \mathrm{C}$ NMR (126 MHz, $\mathrm{CDCl}_{3}$ )

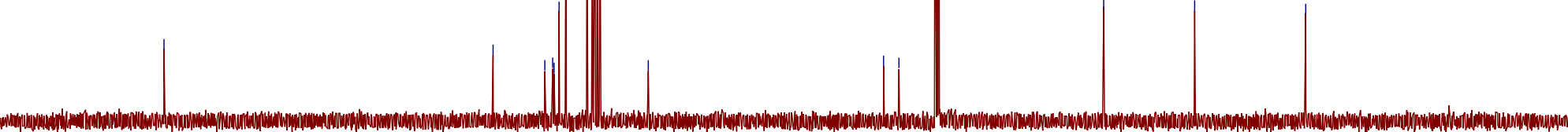

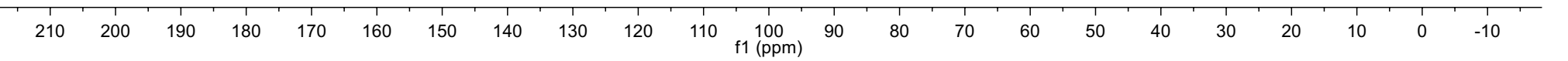




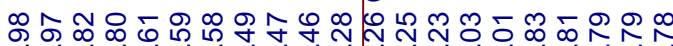

$\sim N N \wedge N N$ N N N N N N N

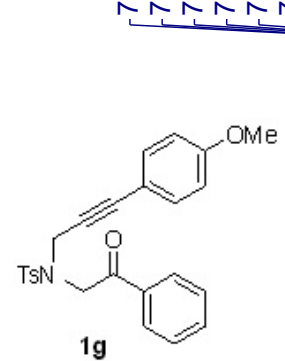

19

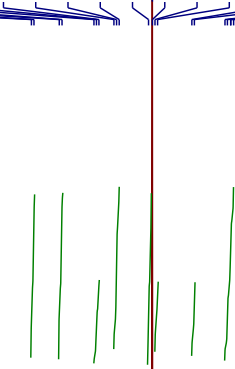

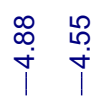

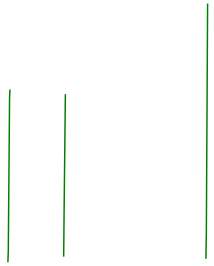

@

I

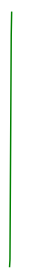

${ }^{1} \mathrm{H} \mathrm{NMR}\left(500 \mathrm{MHz}, \mathrm{CDCl}_{3}\right)$
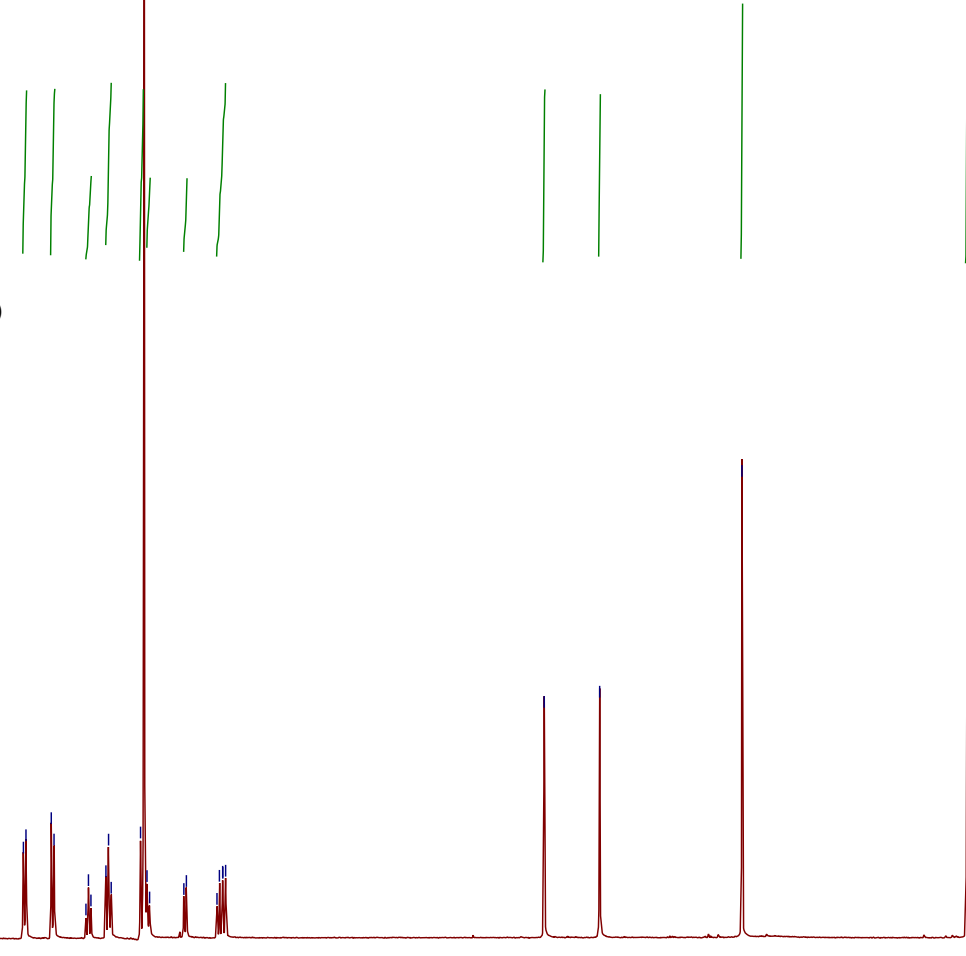

ำ

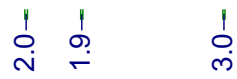

$\stackrel{1}{a}$ 


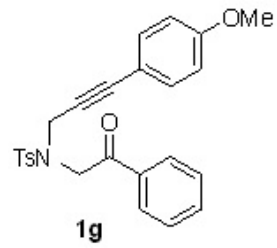

$1 \mathrm{~g}$

${ }^{13} \mathrm{C}$ NMR (126 MHz, $\mathrm{CDCl}_{3}$ )

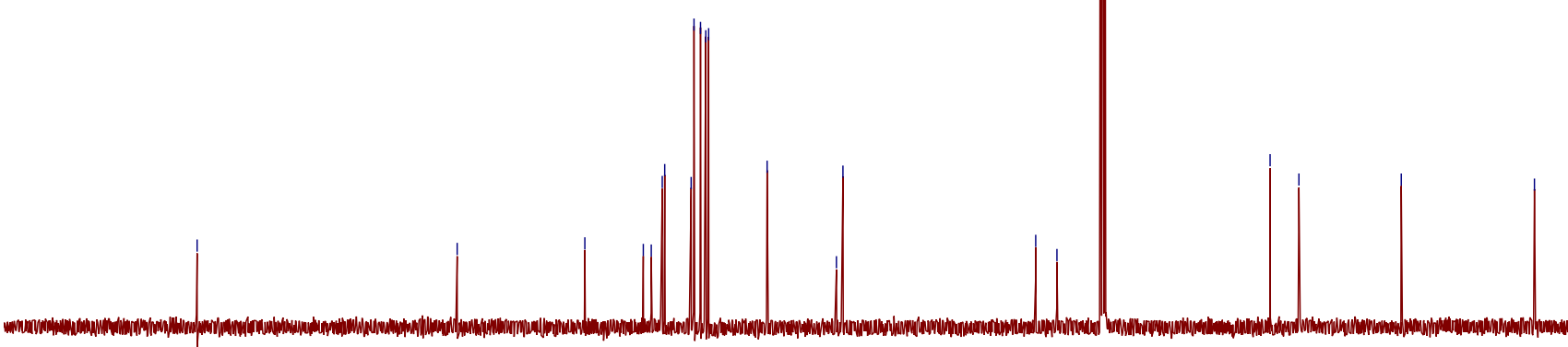

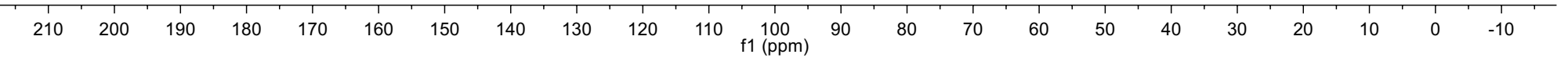


$\frac{m}{0}$

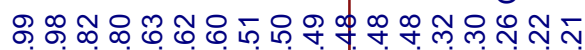

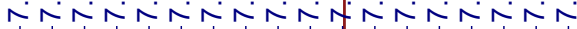

œ

\%

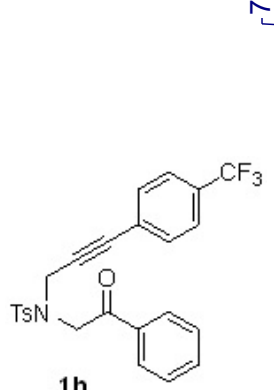

1h

${ }^{1} \mathrm{H} \mathrm{NMR}\left(500 \mathrm{MHz}, \mathrm{CDCl}_{3}\right)$
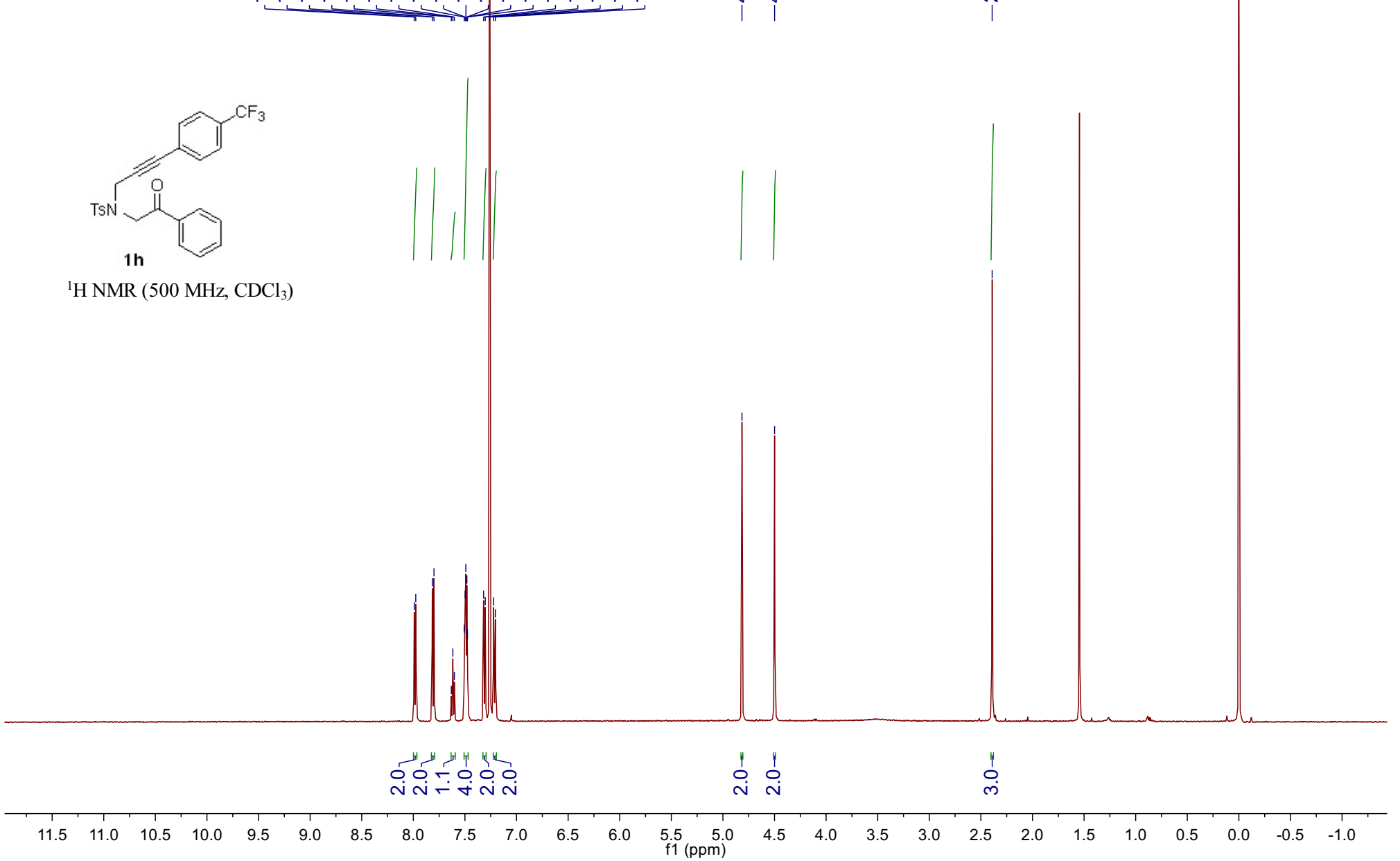


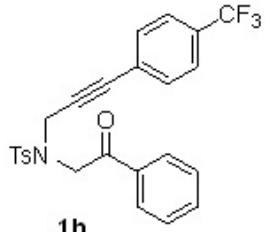

${ }^{13} \mathrm{C}$ NMR (126 MHz, $\mathrm{CDCl}_{3}$ )

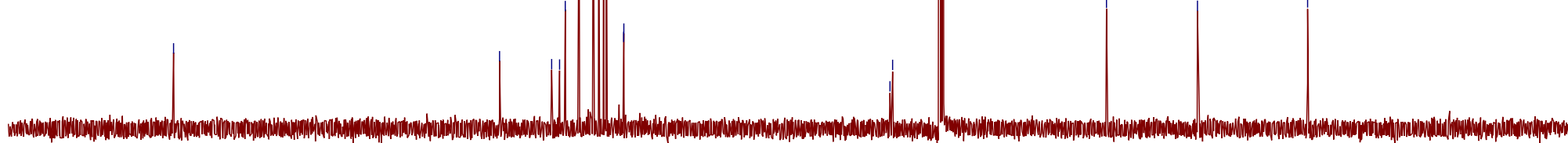

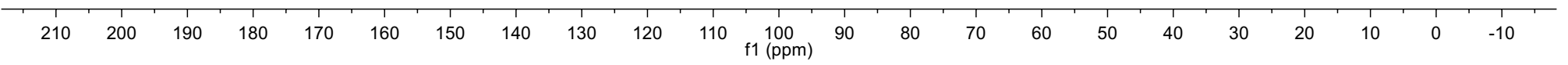




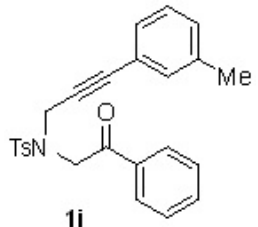

${ }^{1} \mathrm{H} \mathrm{NMR}\left(500 \mathrm{MHz}, \mathrm{CDCl}_{3}\right)$
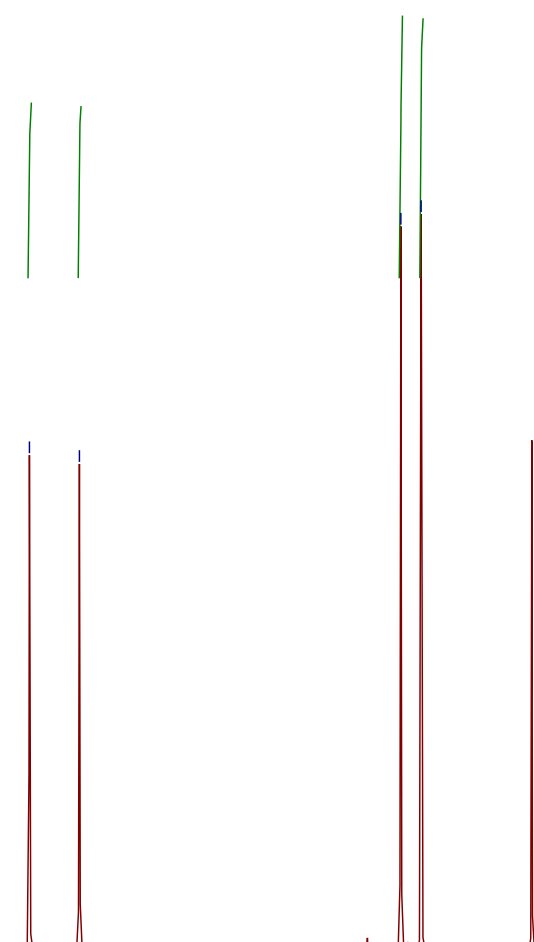

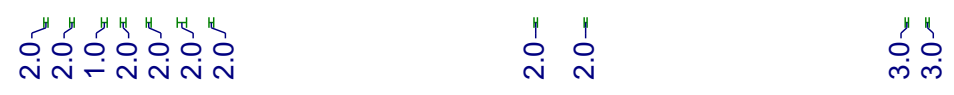




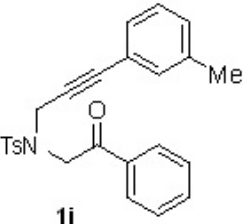

${ }^{13} \mathrm{C}$ NMR $\left(126 \mathrm{MHz}, \mathrm{CDCl}_{3}\right)$ 

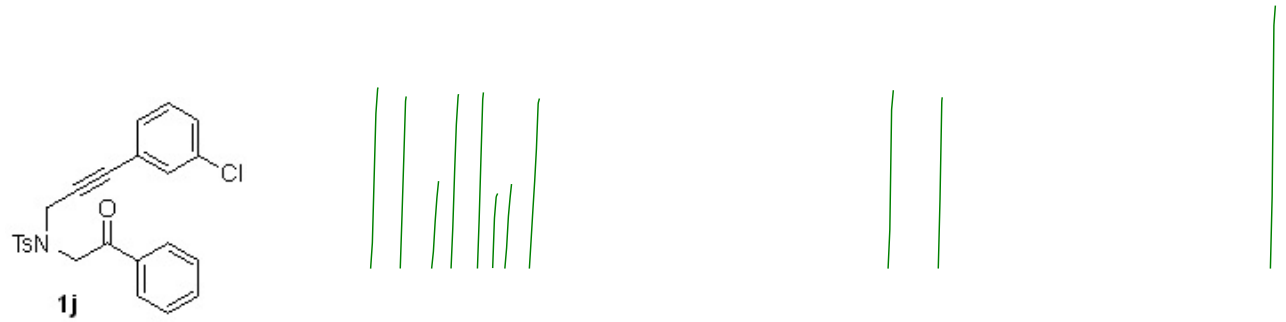

${ }^{1} \mathrm{H}$ NMR (500 MHz, $\mathrm{CDCl}_{3}$ )

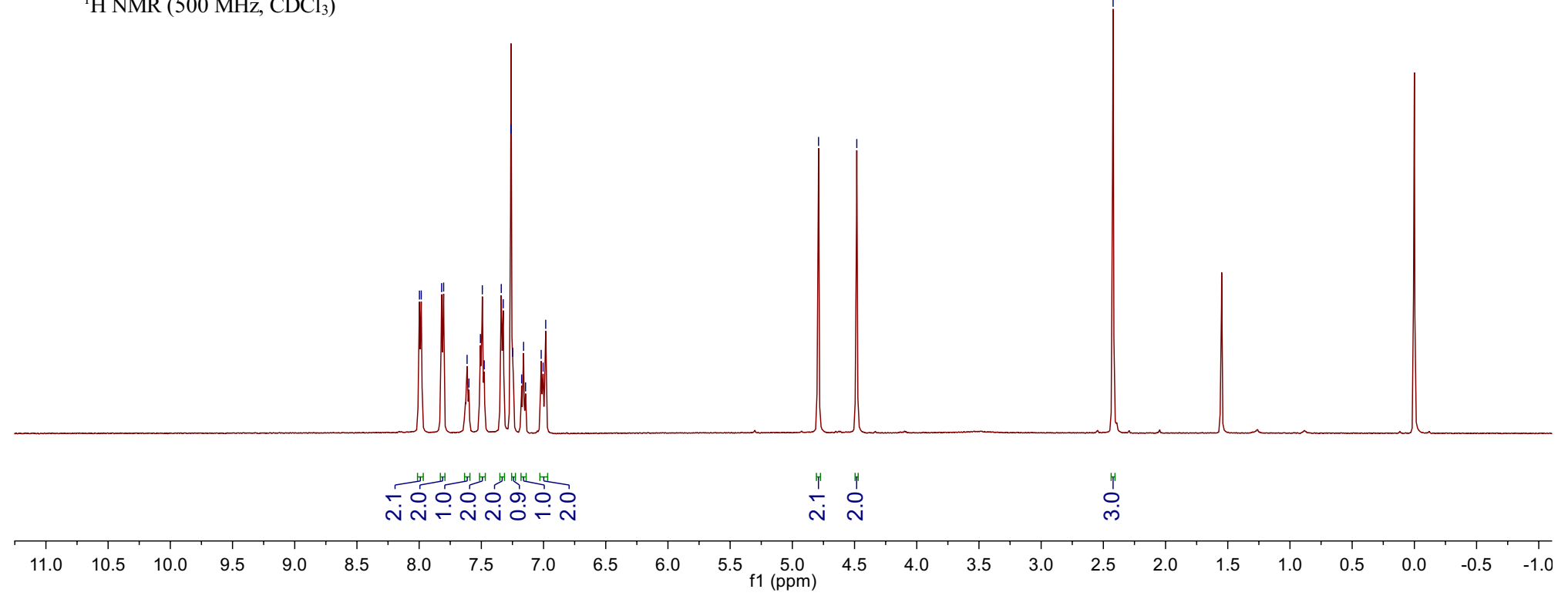




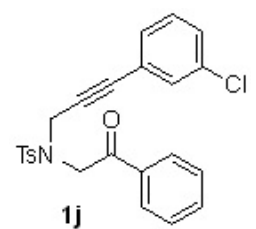

${ }^{13} \mathrm{C} \mathrm{NMR}\left(126 \mathrm{MHz}, \mathrm{CDCl}_{3}\right)$
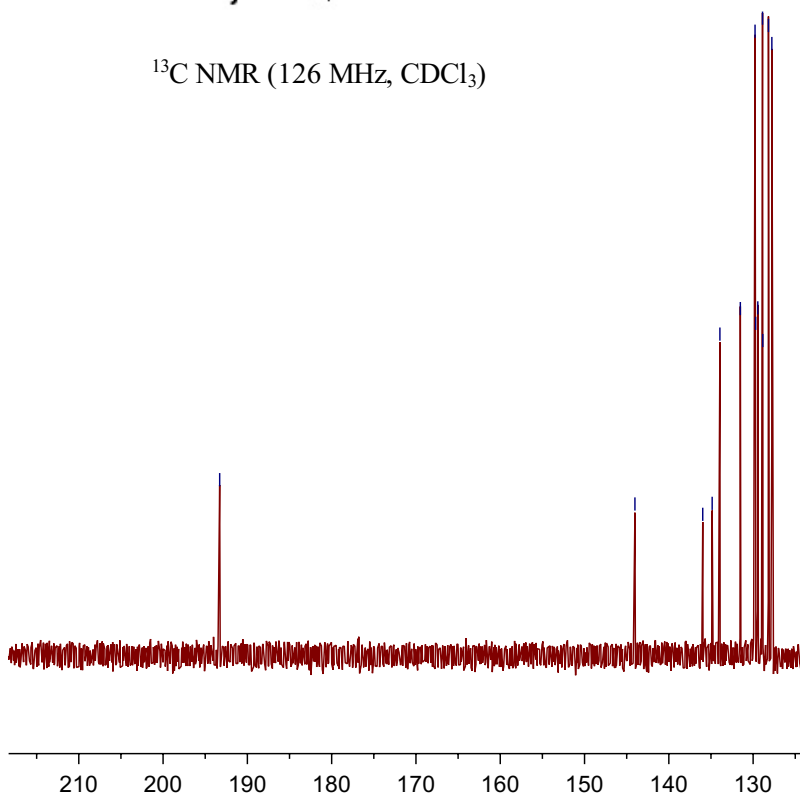


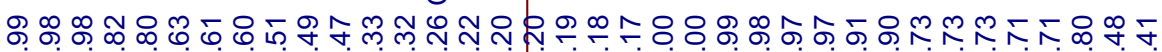

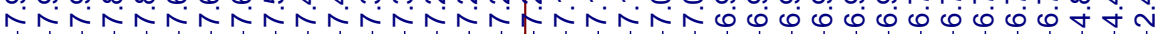

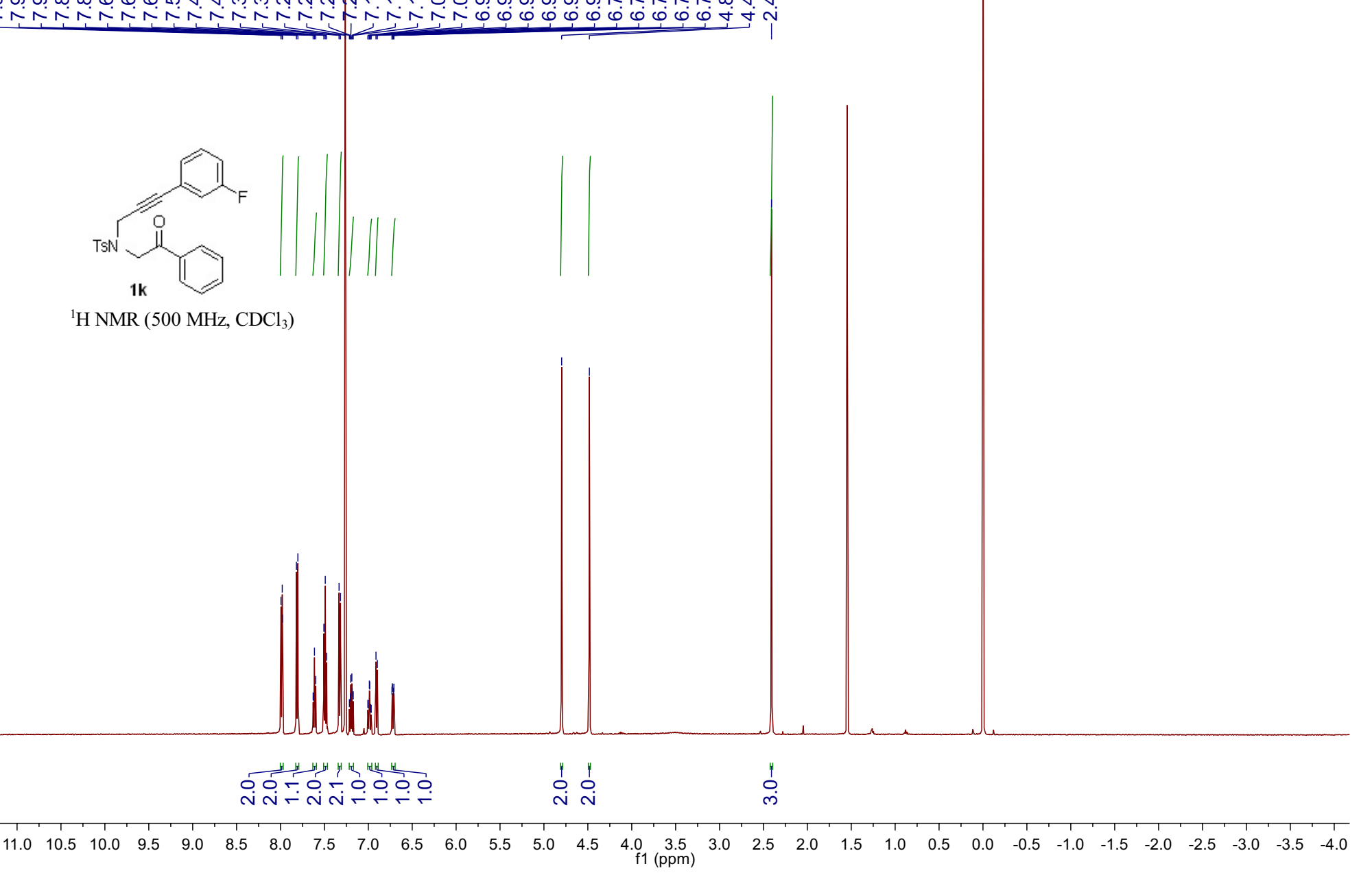


के

ํㅡㄹ

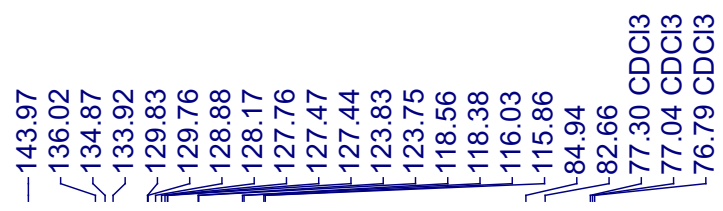

कै

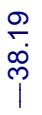

$\stackrel{\infty}{\underset{1}{+}}$<smiles>[13CH3]N(CC#Cc1cccc(F)c1)CC(=O)c1ccccc1</smiles>

${ }^{13} \mathrm{C} \mathrm{NMR}\left(126 \mathrm{MHz}, \mathrm{CDCl}_{3}\right)$

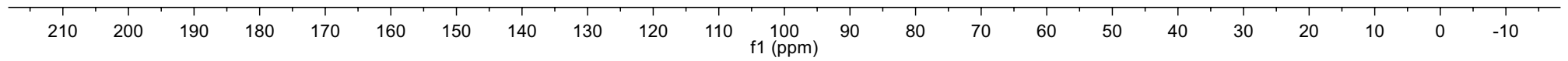




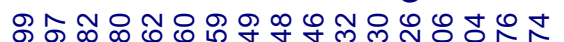

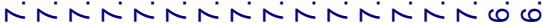

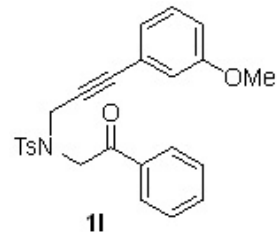

${ }^{1} \mathrm{H} \mathrm{NMR}\left(500 \mathrm{MHz}, \mathrm{CDCl}_{3}\right)$
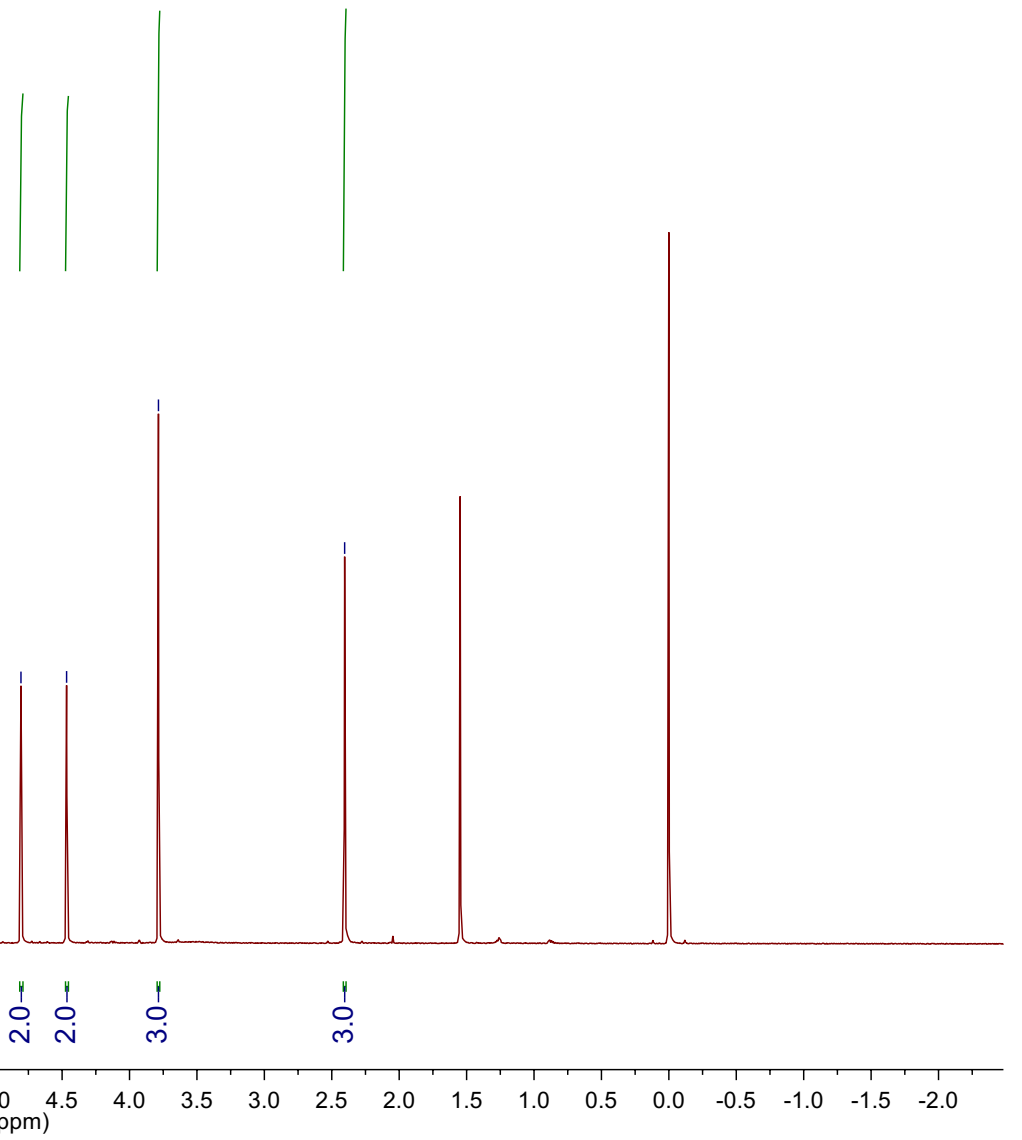


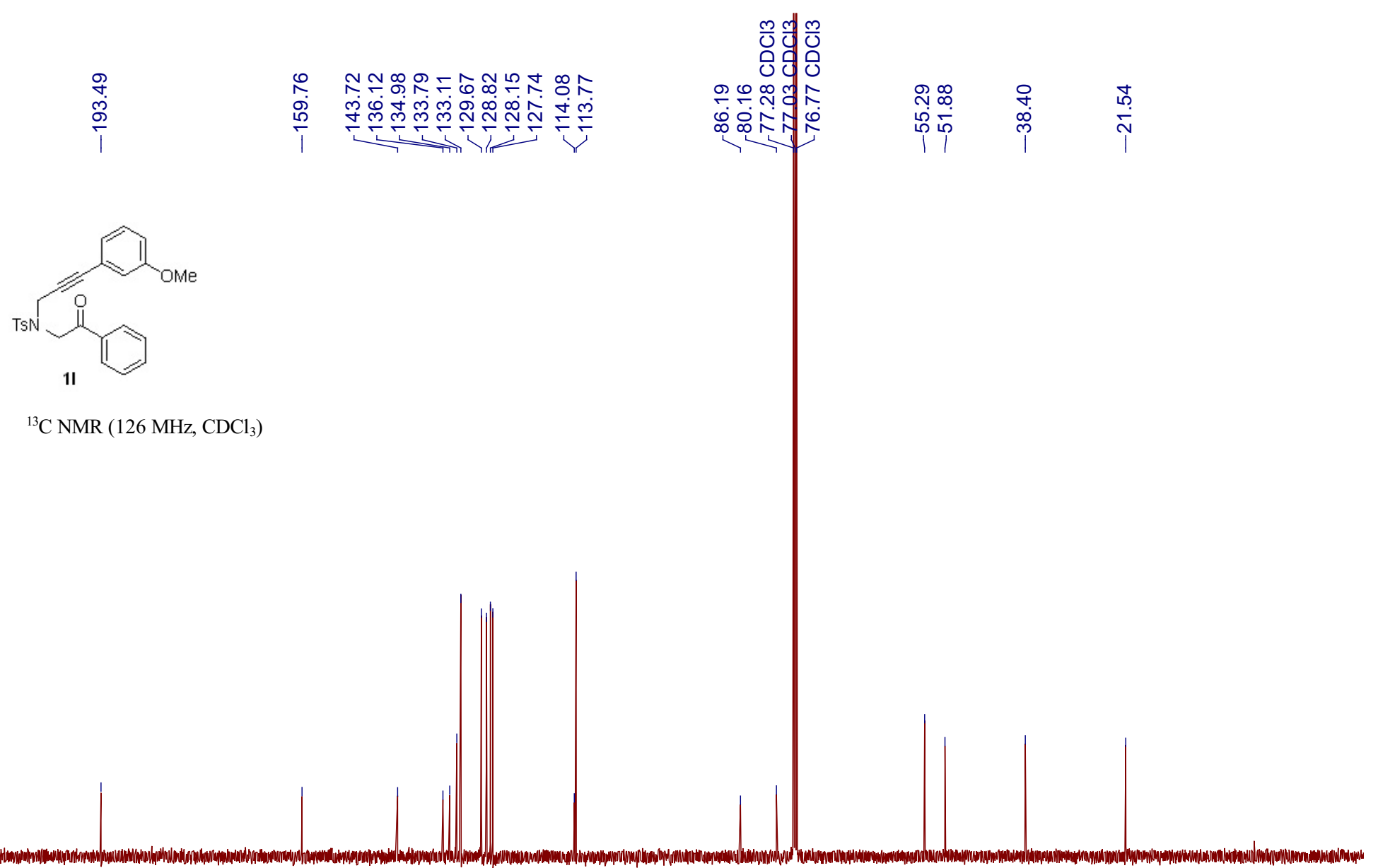

\begin{tabular}{|c|c|c|c|c|c|c|c|c|c|c|c|c|c|c|c|c|c|c|c|c|c|c|}
\hline 1 & 1 & 1 & $T$ & T & $T$ & $T$ & $T$ & $T$ & 1 & $T$ & 1 & 1 & 1 & 1 & $T$ & 1 & 1 & $T$ & 1 & 1 & 1 & $T$ \\
\hline 210 & 200 & 190 & 180 & 170 & 160 & 150 & 140 & 130 & 120 & 110 & $\begin{array}{c}100 \\
\mathrm{f} 1(\mathrm{ppm})\end{array}$ & 90 & 80 & 70 & 60 & 50 & 40 & 30 & 20 & 10 & 0 & -10 \\
\hline
\end{tabular}



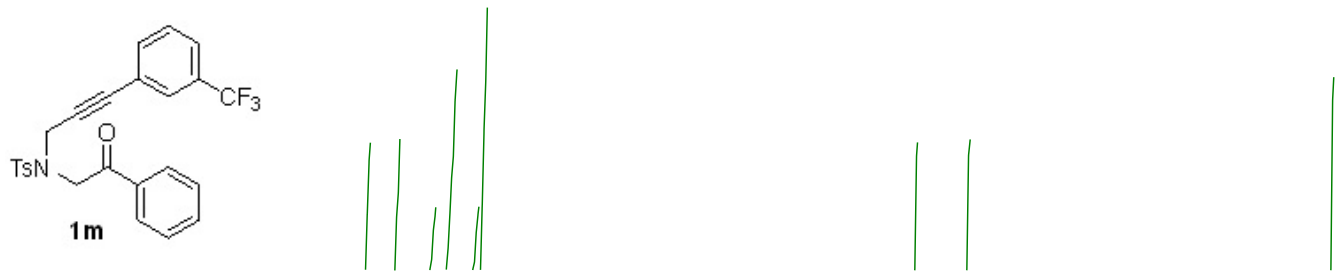

${ }^{1} \mathrm{H} \mathrm{NMR}\left(500 \mathrm{MHz}, \mathrm{CDCl}_{3}\right)$

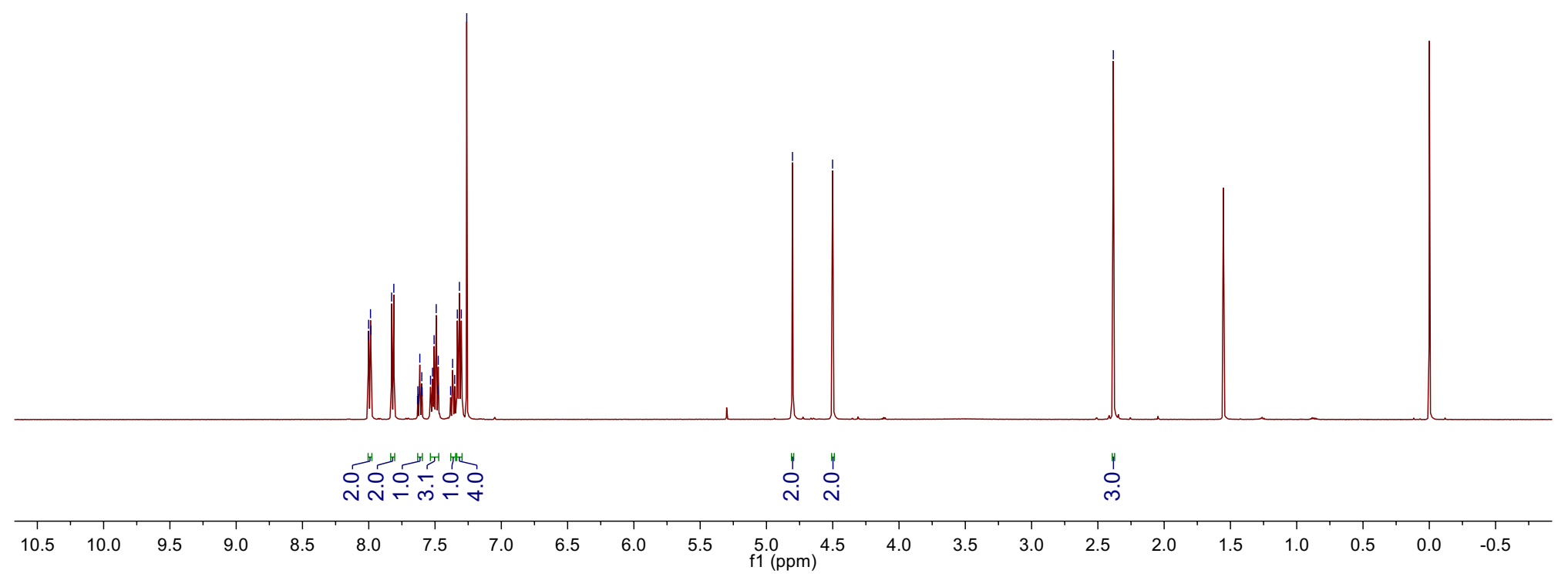




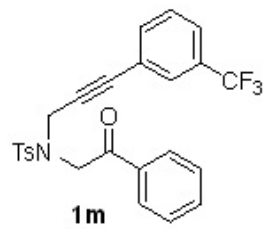

${ }^{13} \mathrm{C}$ NMR $\left(126 \mathrm{MHz}, \mathrm{CDCl}_{3}\right)$

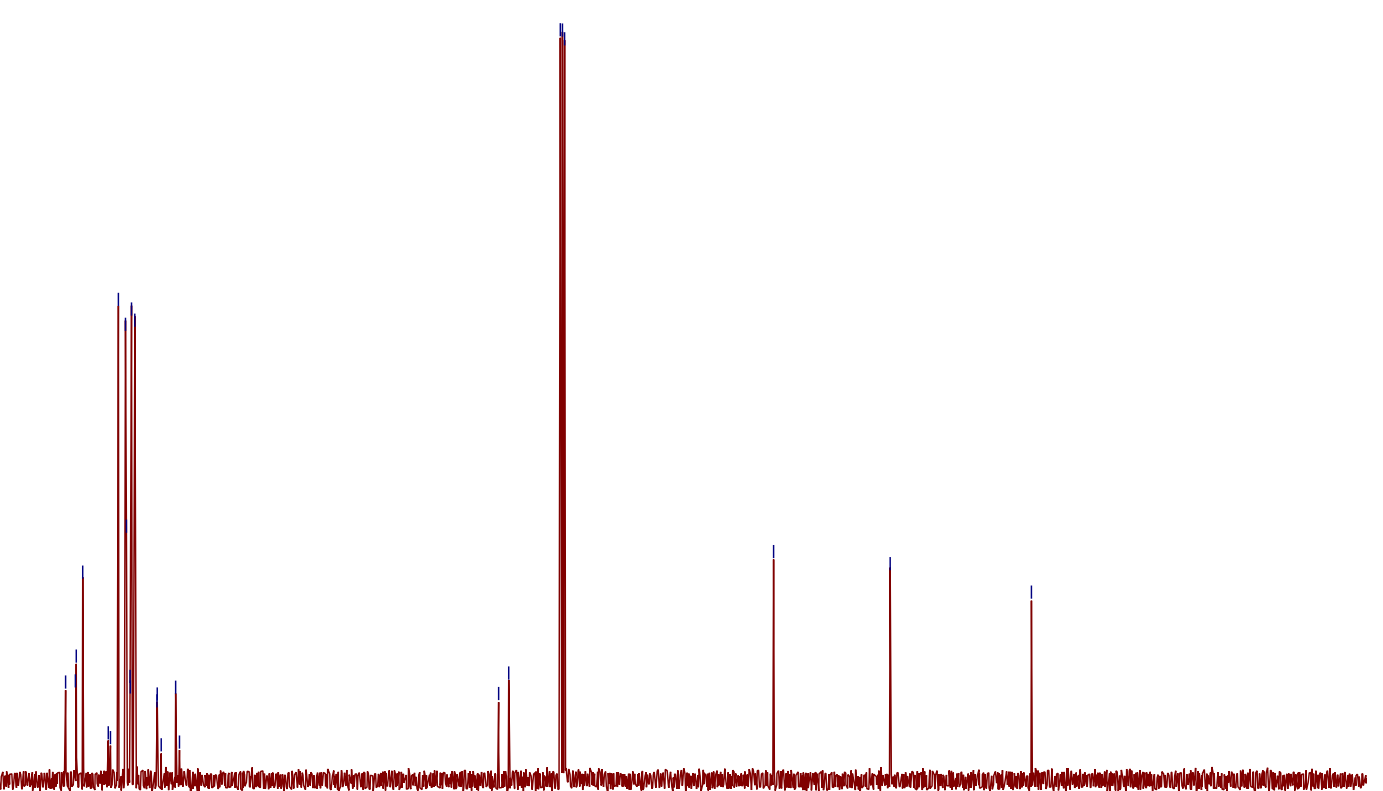


${ }^{1} \mathrm{H} \mathrm{NMR}\left(500 \mathrm{MHz}, \mathrm{CDCl}_{3}\right)$
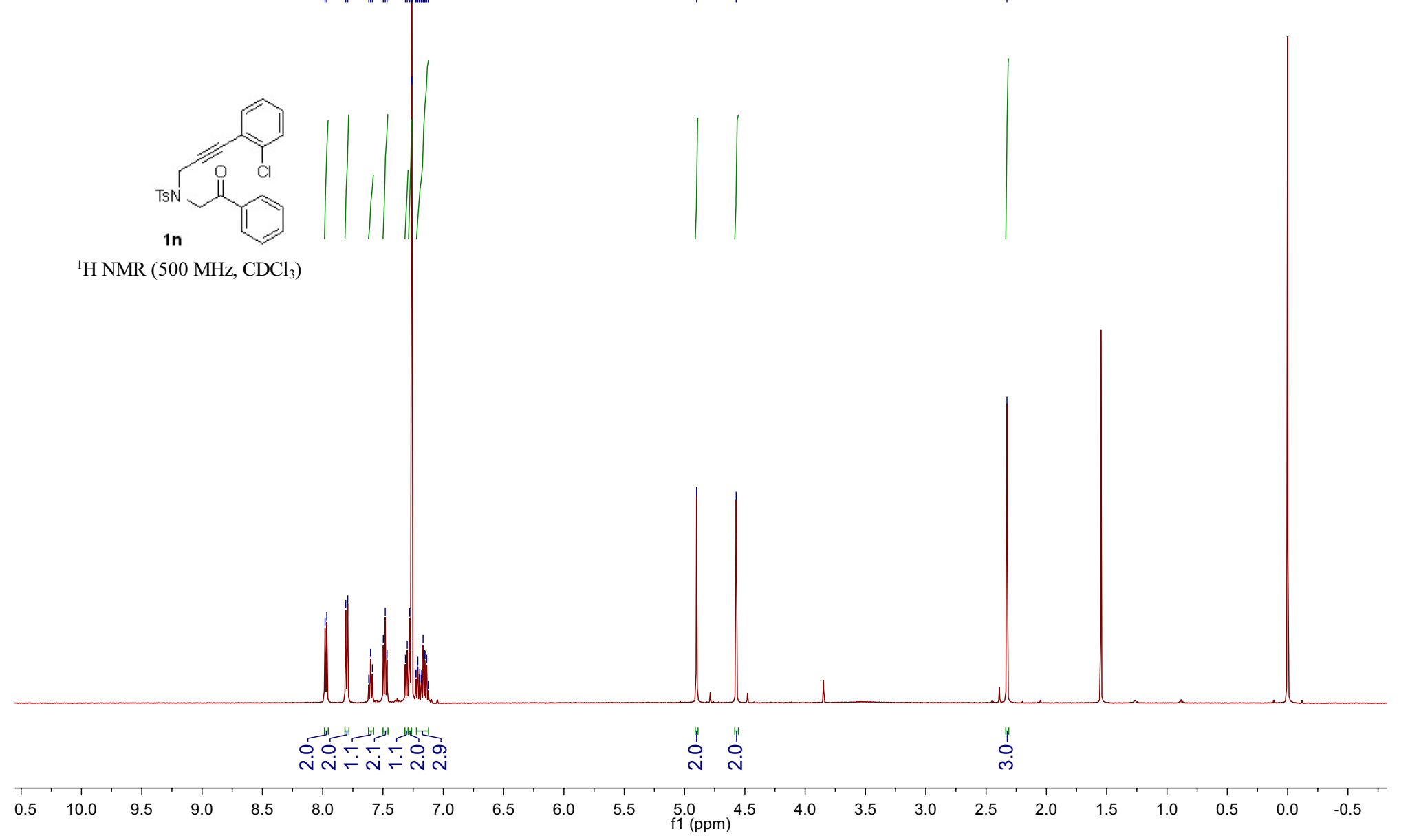


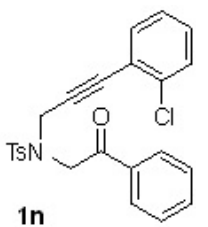

${ }^{13} \mathrm{C}$ NMR $\left(126 \mathrm{MHz}, \mathrm{CDCl}_{3}\right)$

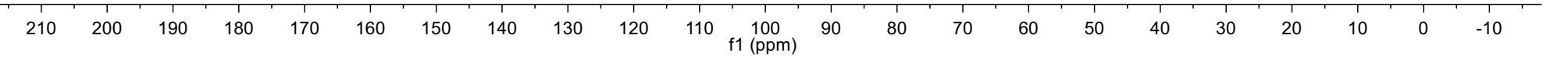




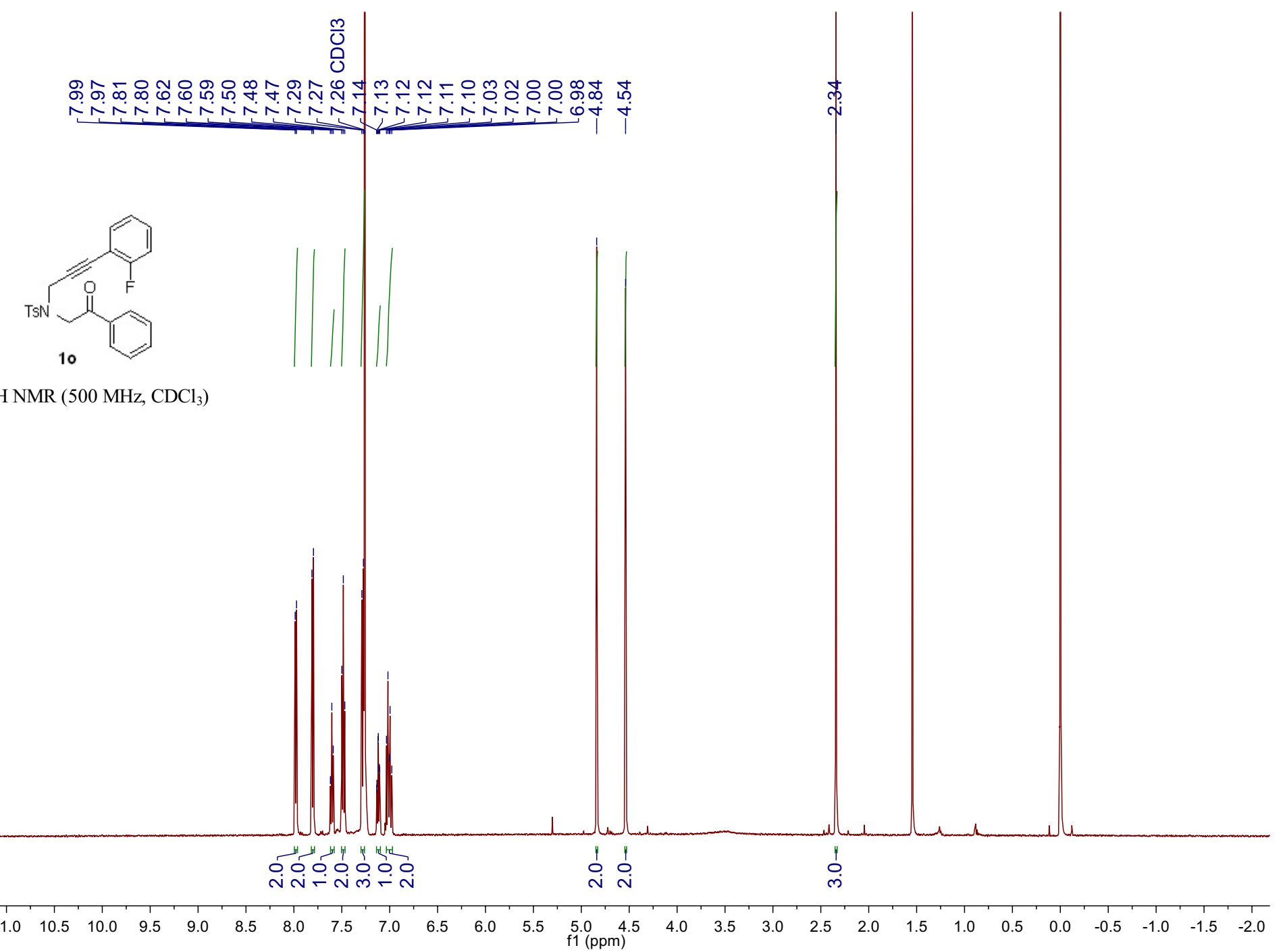


ले

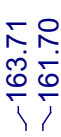

$\frac{1}{i}$

$\underset{\infty}{\infty}$

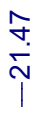<smiles></smiles>

${ }^{13} \mathrm{C}$ NMR (126 MHz, $\mathrm{CDCl}_{3}$ )

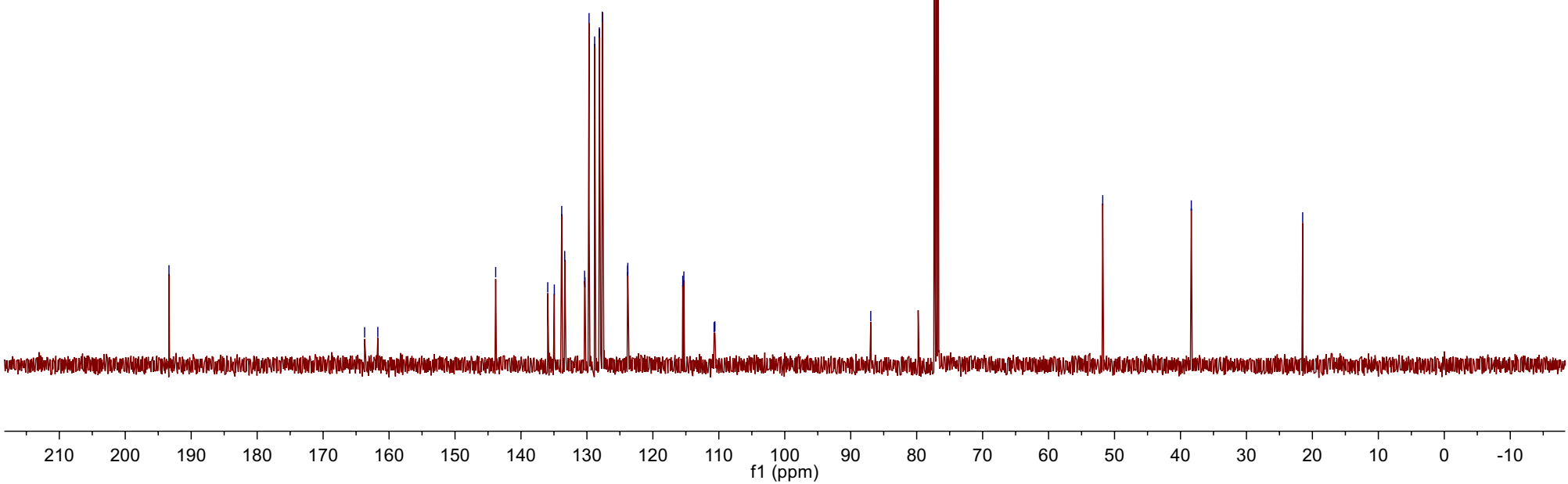




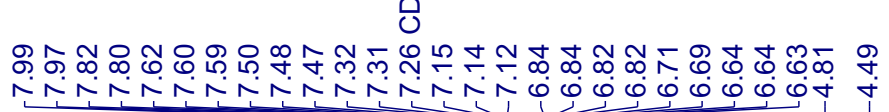<smiles>COc1ccccc1C#CCNCC(=O)c1ccccc1</smiles>

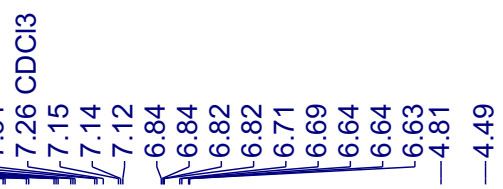

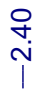

$1 \mathrm{p}$
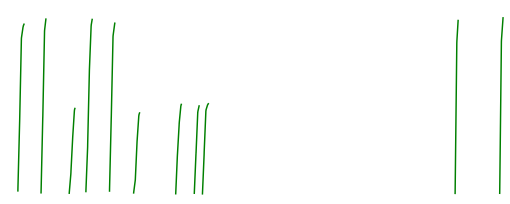

${ }^{1} \mathrm{H} \mathrm{NMR}\left(500 \mathrm{MHz}, \mathrm{CDCl}_{3}\right.$ )
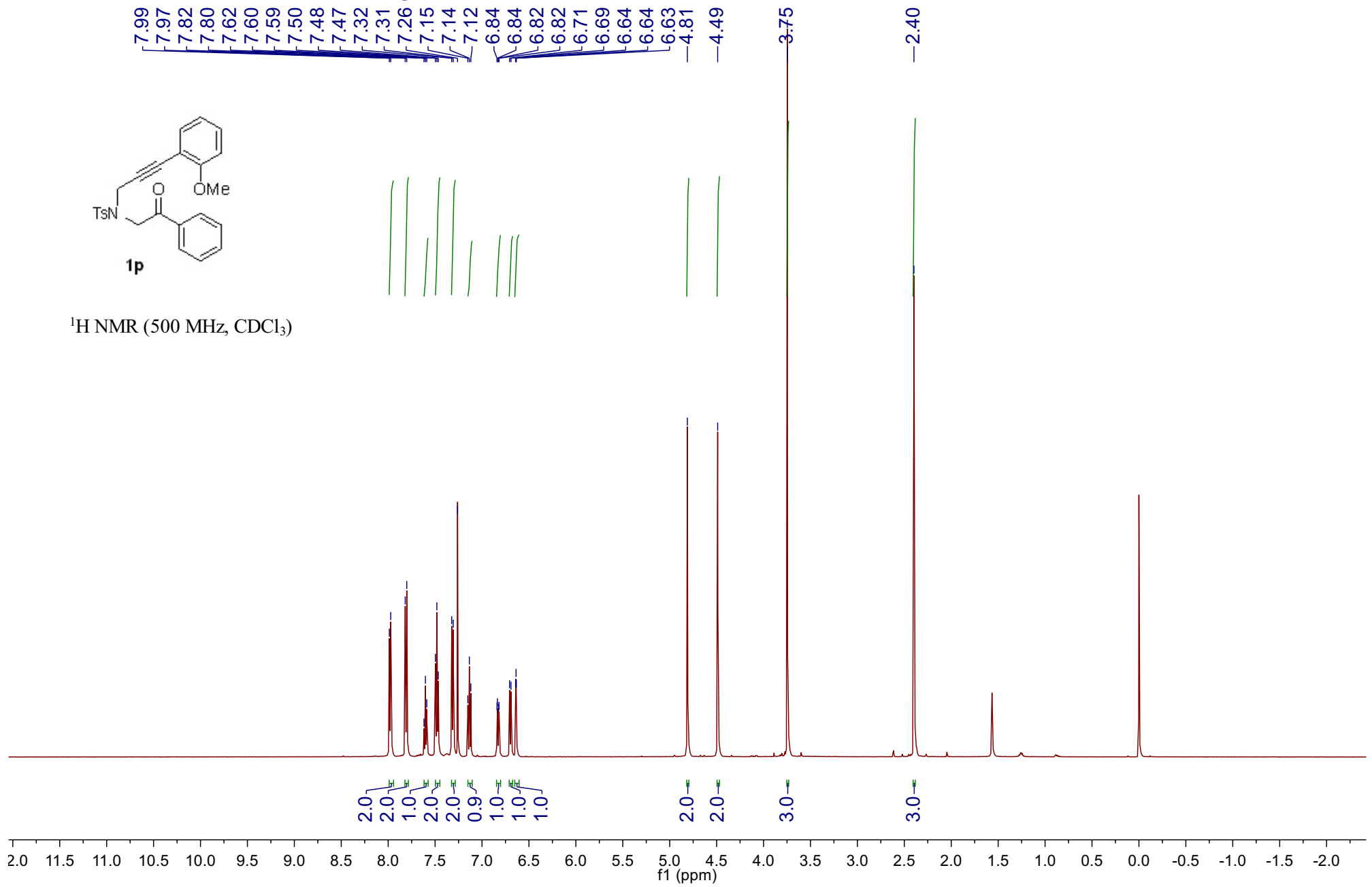


\begin{tabular}{|c|c|c|c|c|c|}
\hline $\begin{array}{l}\stackrel{9}{0} \\
\text { ’ }\end{array}$ & 衤 & 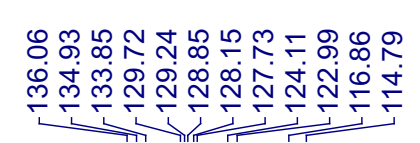 & 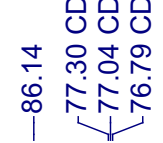 & 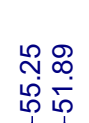 & 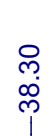 \\
\hline
\end{tabular}

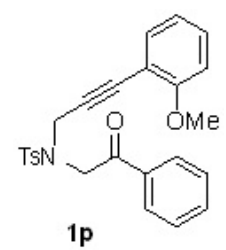

${ }^{13} \mathrm{C}$ NMR (126 MHz, $\left.\mathrm{CDCl}_{3}\right)$

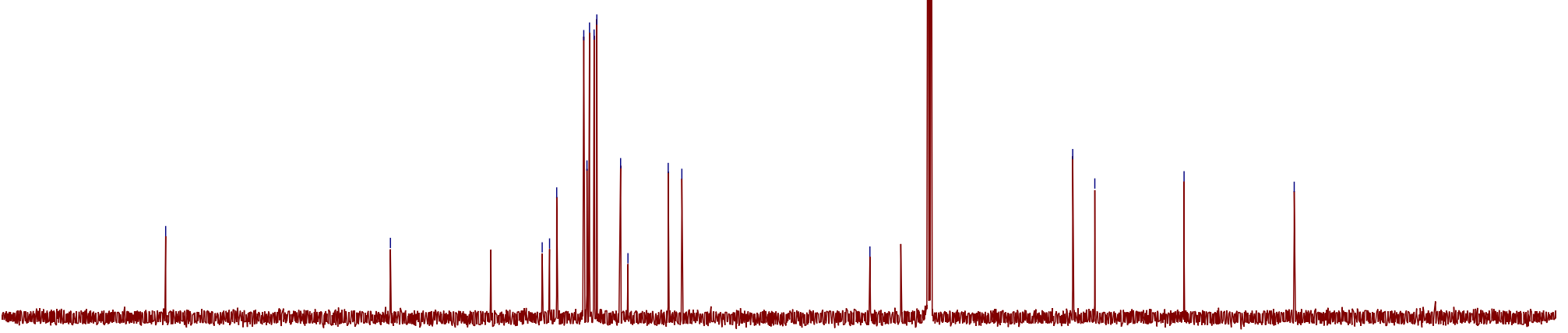

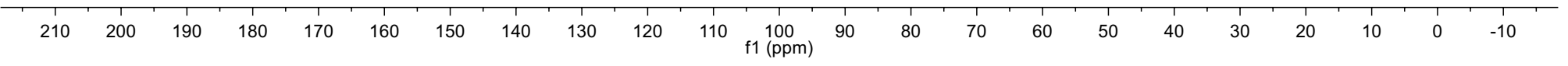



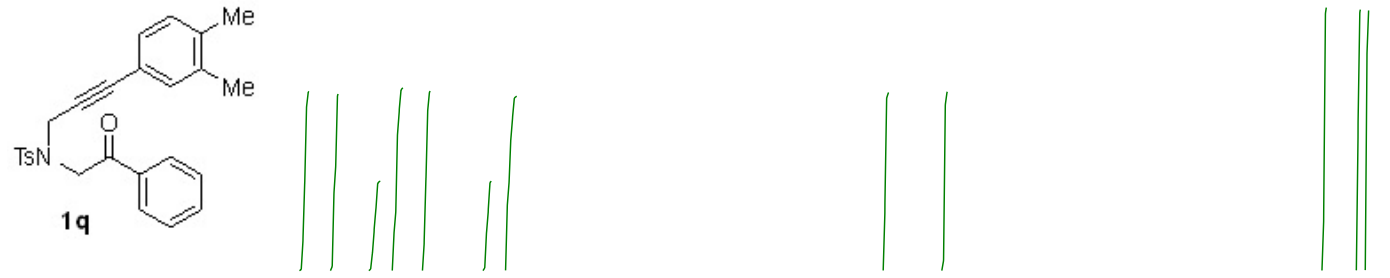

${ }^{1} \mathrm{H}$ NMR (500 MHz, $\mathrm{CDCl}_{3}$ )

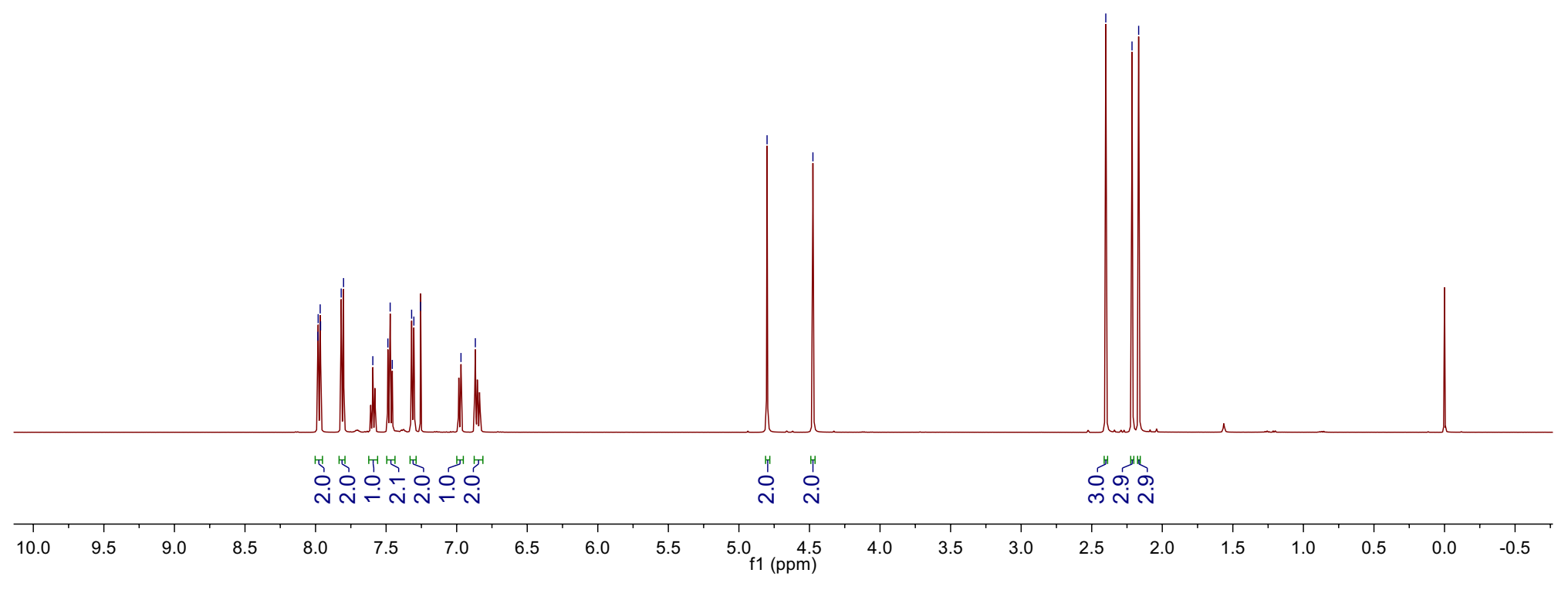




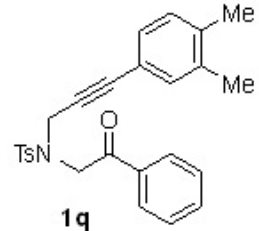

${ }^{13} \mathrm{C}$ NMR $\left(126 \mathrm{MHz}, \mathrm{CDCl}_{3}\right)$

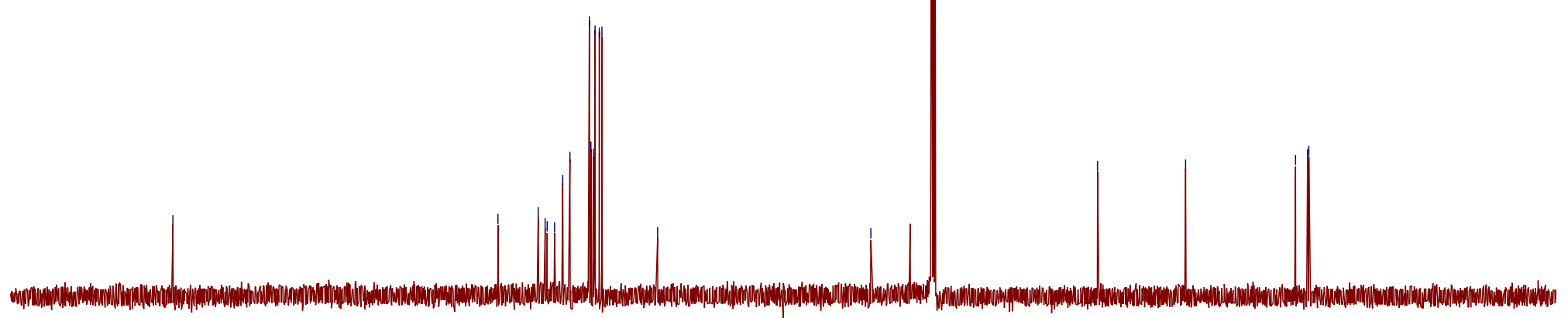

\begin{tabular}{|c|c|c|c|c|c|c|c|c|c|c|c|c|c|c|c|c|c|c|c|c|c|c|}
\hline 1 & 1 & 1 & 1 & $T$ & 1 & 1 & $T$ & 1 & 1 & 1 & 1 & 1 & 1 & 1 & 1 & 1 & 1 & 1 & 1 & 1 & 1 & 1 \\
\hline 210 & 200 & 190 & 180 & 170 & 160 & 150 & 140 & 130 & 120 & 110 & $\begin{array}{c}100 \\
\mathrm{f} 1(\mathrm{ppm})\end{array}$ & 90 & 80 & 70 & 60 & 50 & 40 & 30 & 20 & 10 & 0 & -10 \\
\hline
\end{tabular}




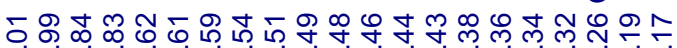

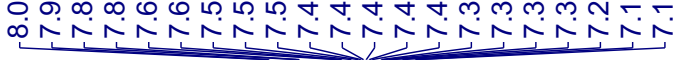

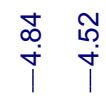

$\stackrel{\substack{+i}}{i}$
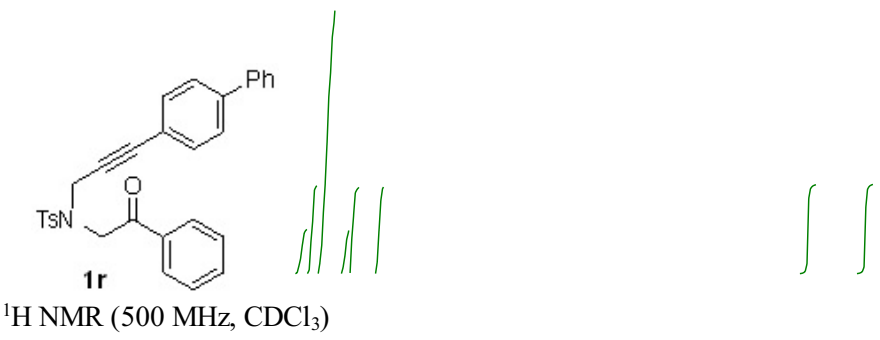

${ }^{1} \mathrm{H}$ NMR (500 MHz, $\mathrm{CDCl}_{3}$ )

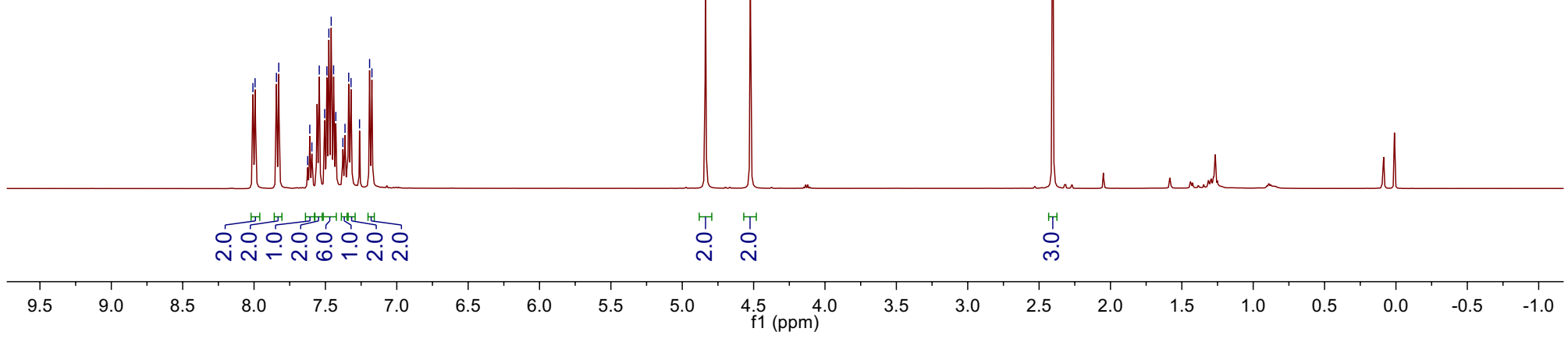




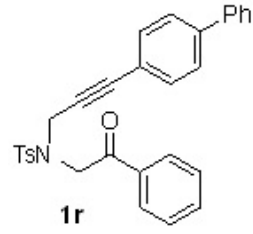

${ }^{13} \mathrm{C}$ NMR $\left(126 \mathrm{MHz}, \mathrm{CDCl}_{3}\right)$

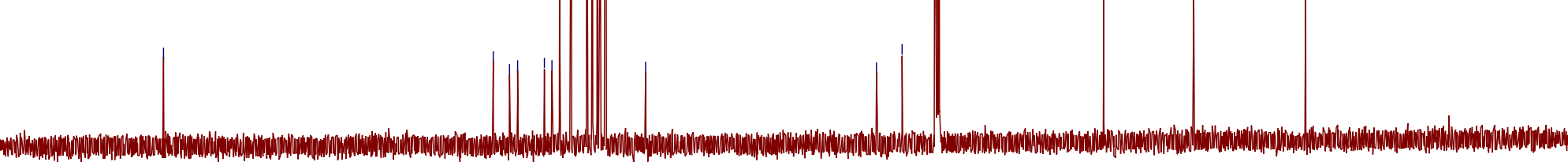

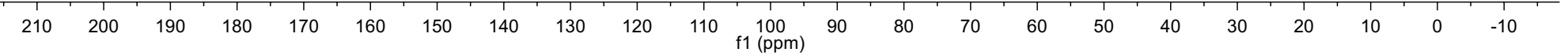




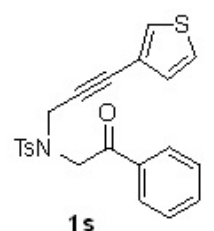

$1 \mathrm{~s}$

${ }^{1} \mathrm{H}$ NMR $\left(500 \mathrm{MHz}, \mathrm{CDCl}_{3}\right)$
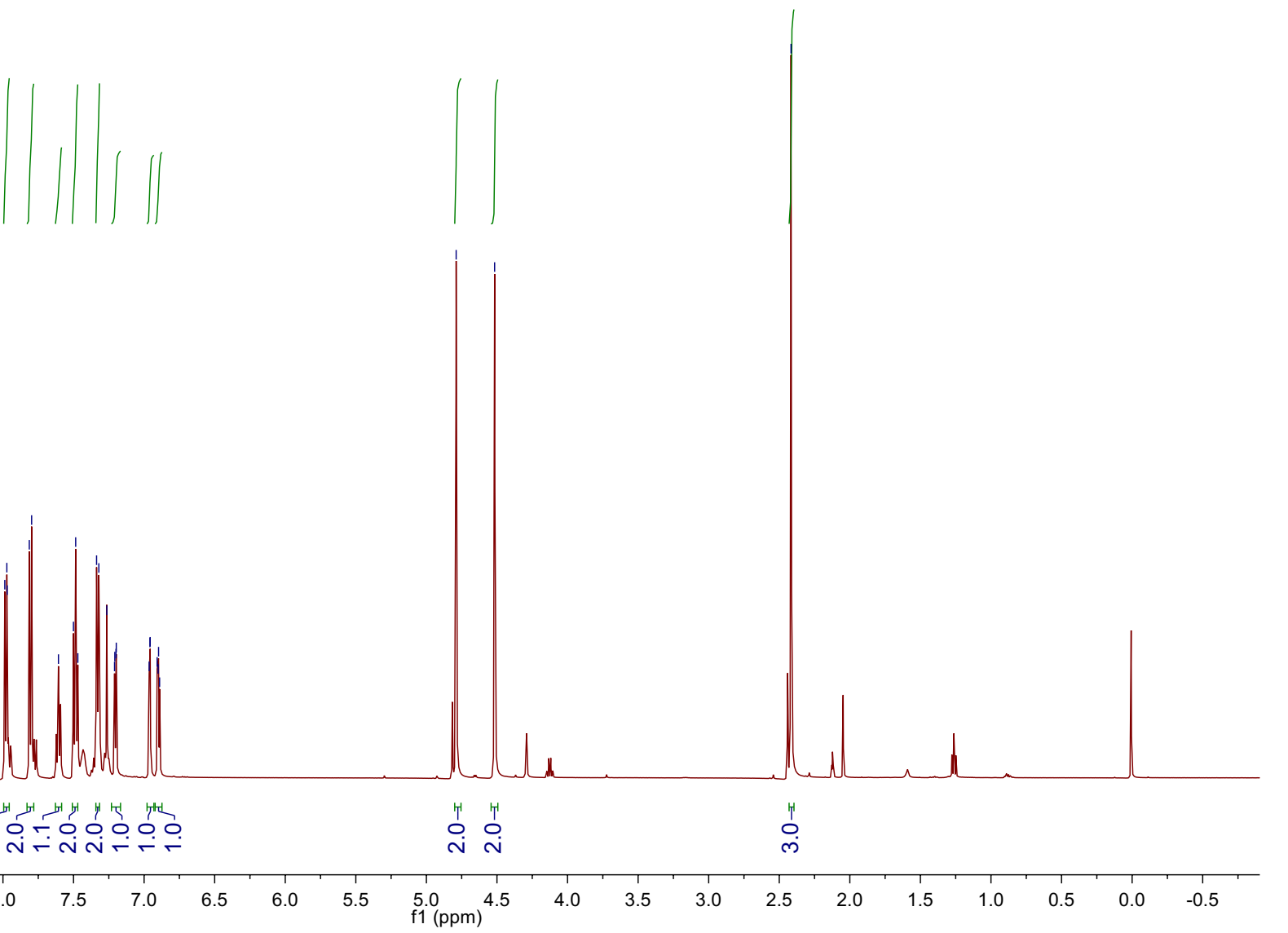


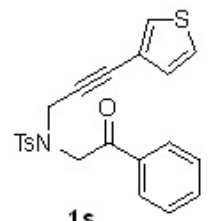

15

${ }^{13} \mathrm{C}$ NMR (126 MHz, $\left.\mathrm{CDCl}_{3}\right)$

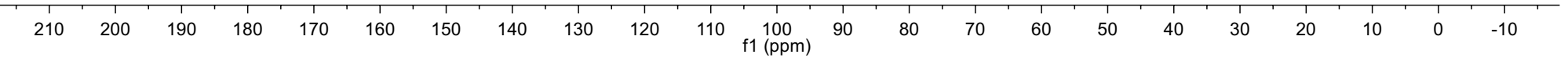


ญ

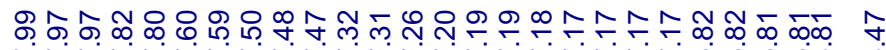

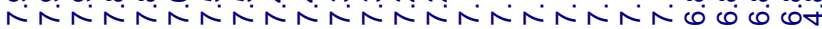
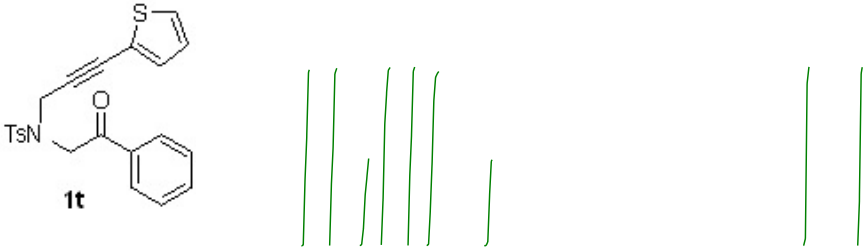

${ }^{1} \mathrm{H} \mathrm{NMR}\left(500 \mathrm{MHz}, \mathrm{CDCl}_{3}\right)$

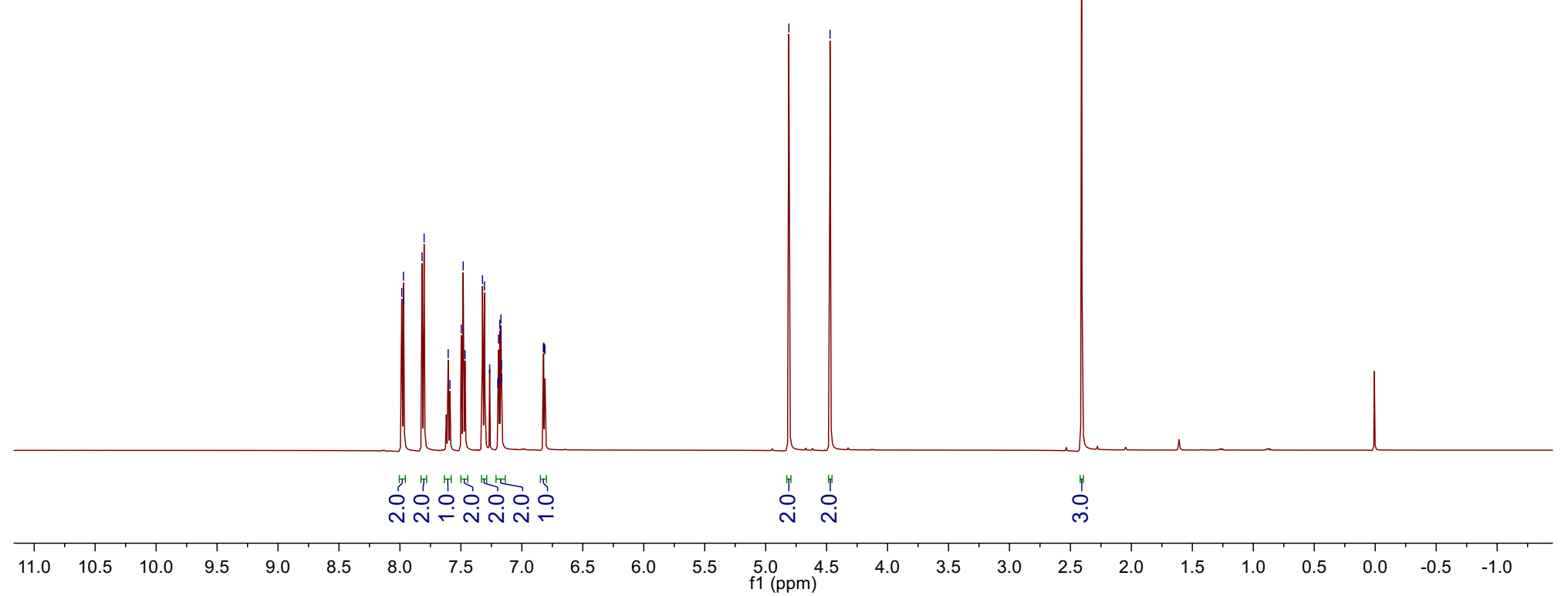




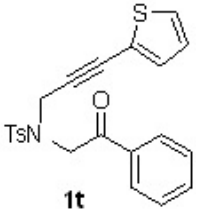

${ }^{13} \mathrm{C}$ NMR $\left(126 \mathrm{MHz}, \mathrm{CDCl}_{3}\right)$
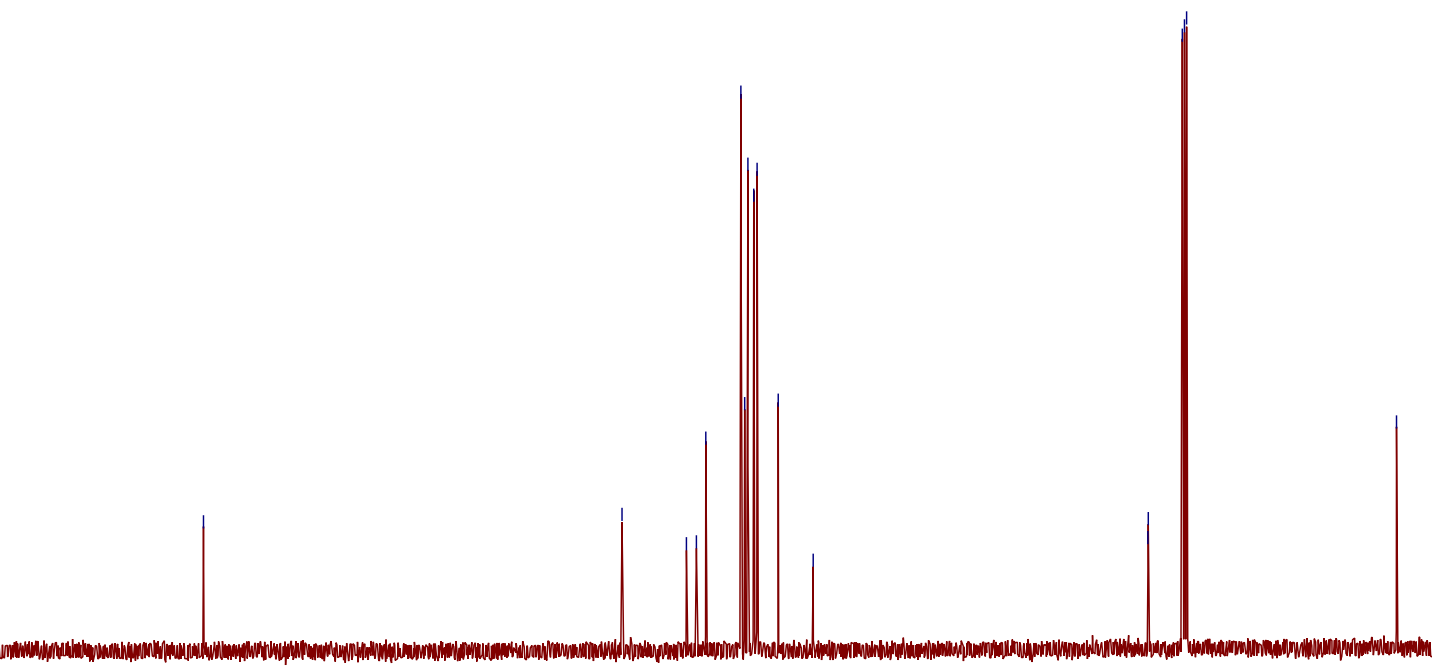

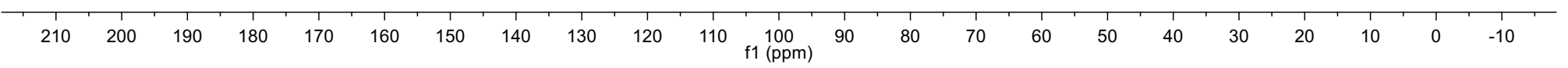



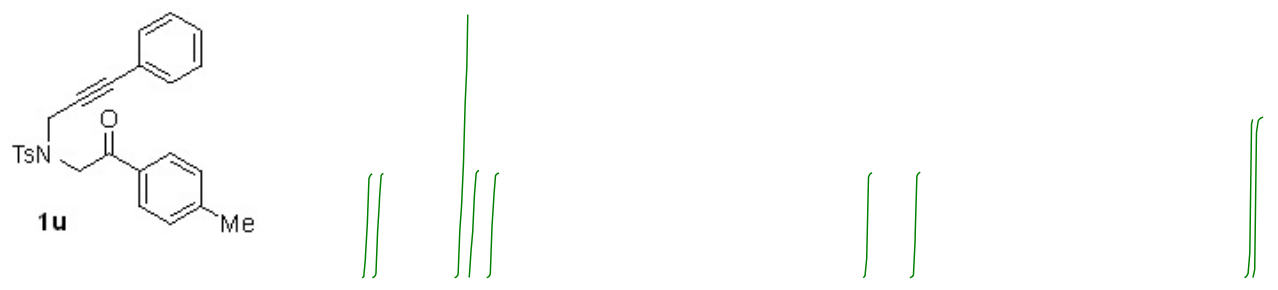

${ }^{1} \mathrm{H} \mathrm{NMR}\left(500 \mathrm{MHz}, \mathrm{CDCl}_{3}\right)$

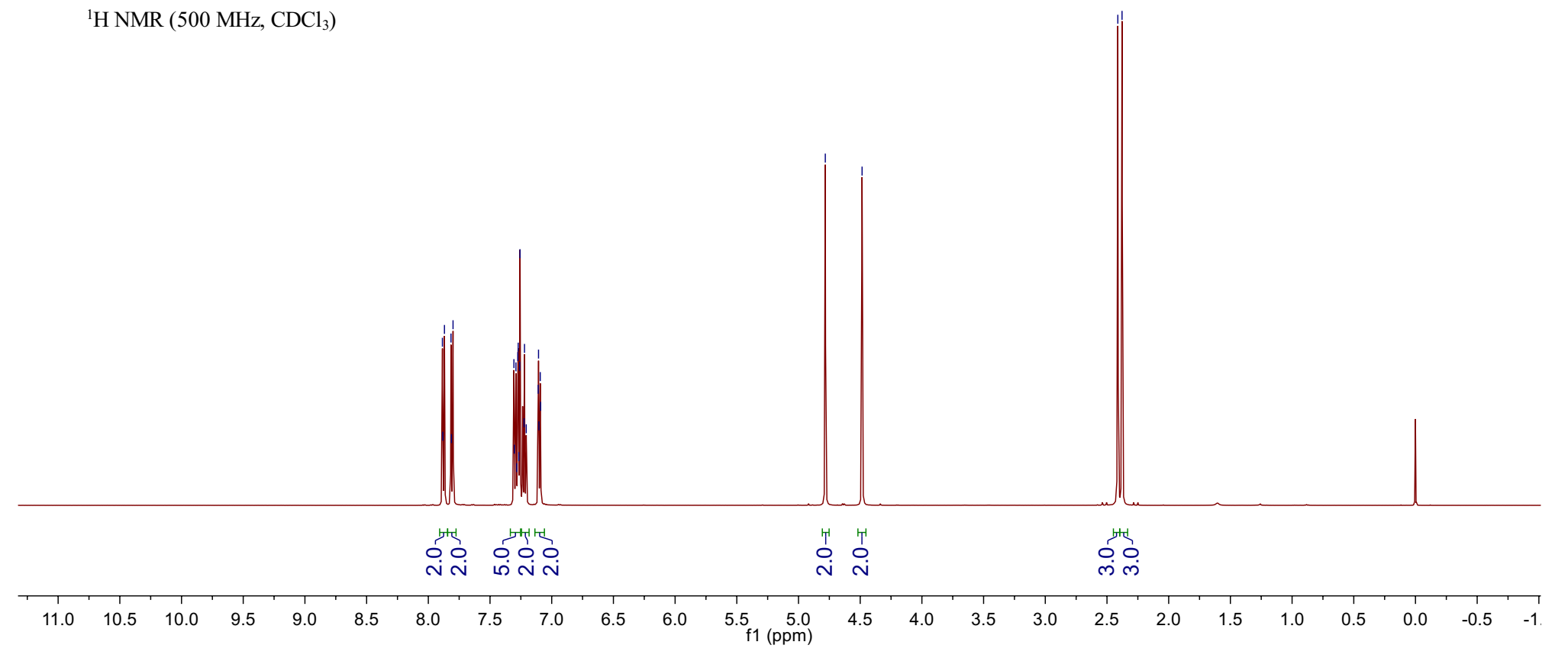




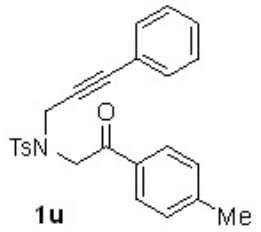

${ }^{13} \mathrm{C}$ NMR $\left(126 \mathrm{MHz}, \mathrm{CDCl}_{3}\right)$

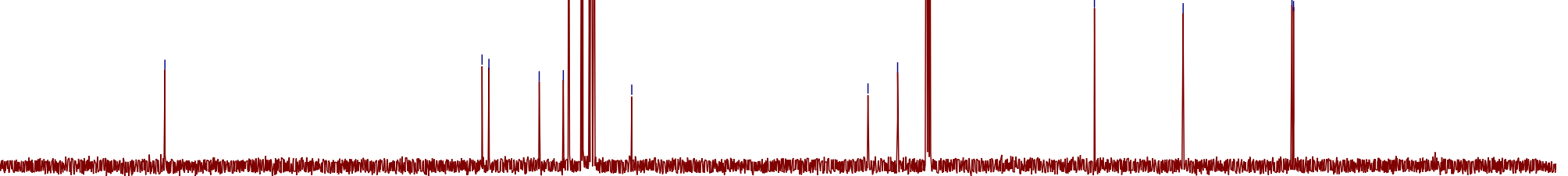




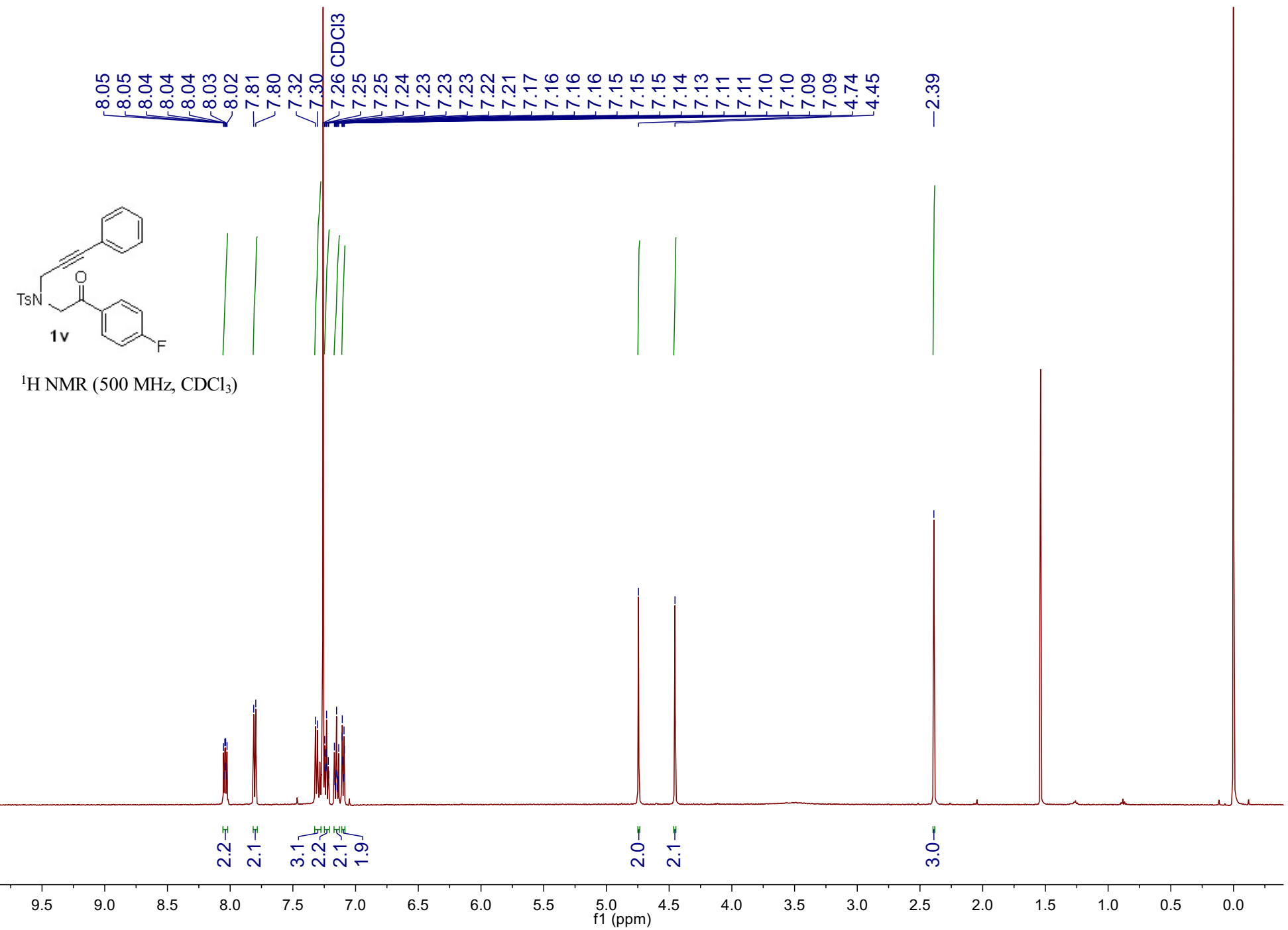


$\frac{m}{0}$

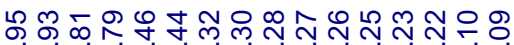

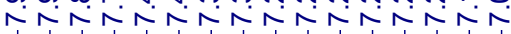

ป

@ి

广

${ }^{1} \mathrm{H} \mathrm{NMR}\left(500 \mathrm{MHz}, \mathrm{CDCl}_{3}\right)$
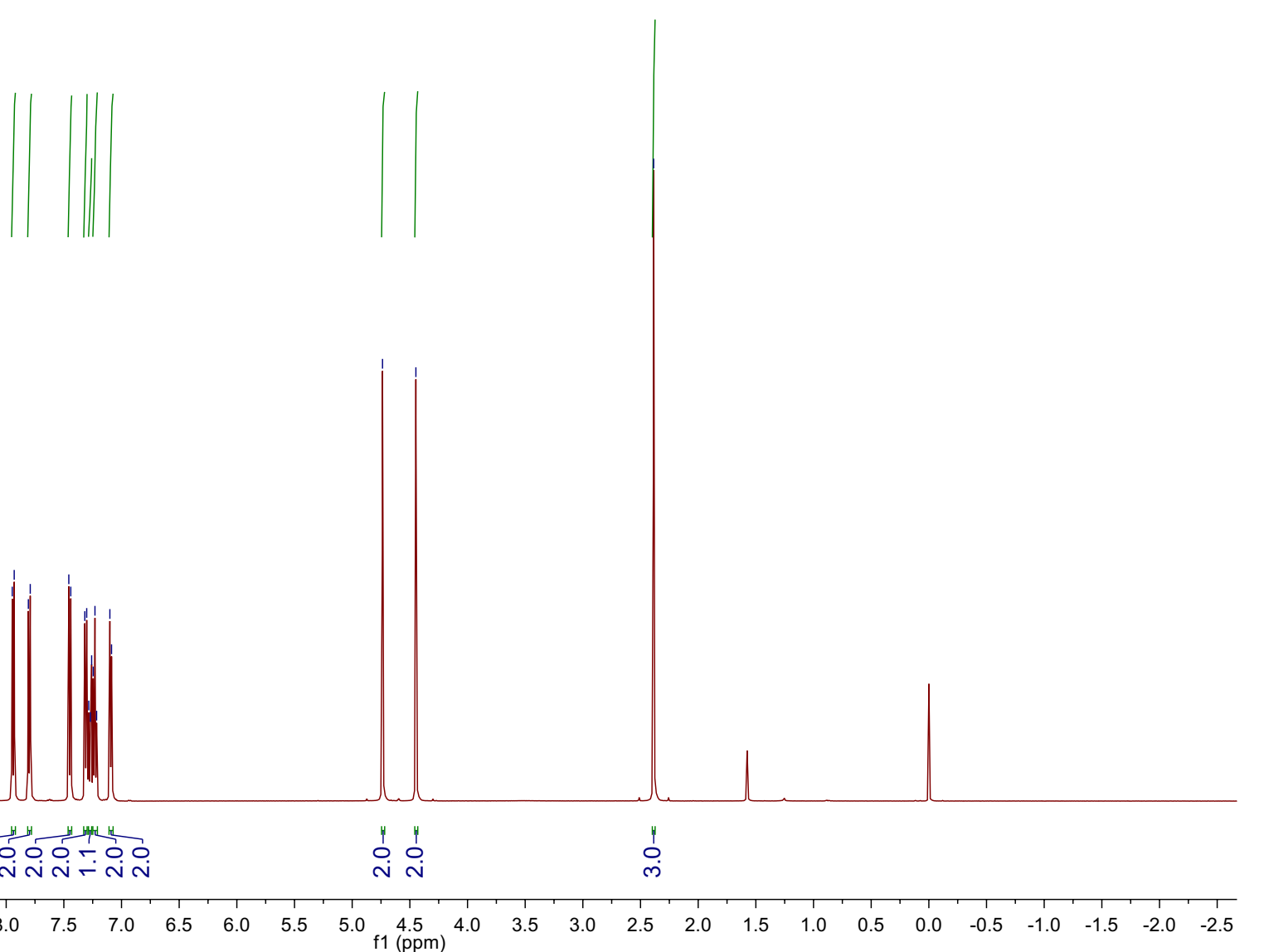


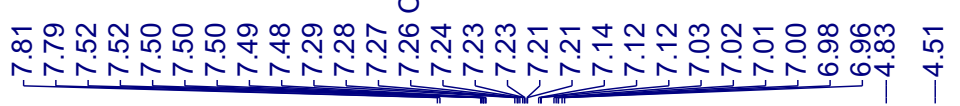

চ్

$\stackrel{\infty}{\stackrel{\infty}{i}}$

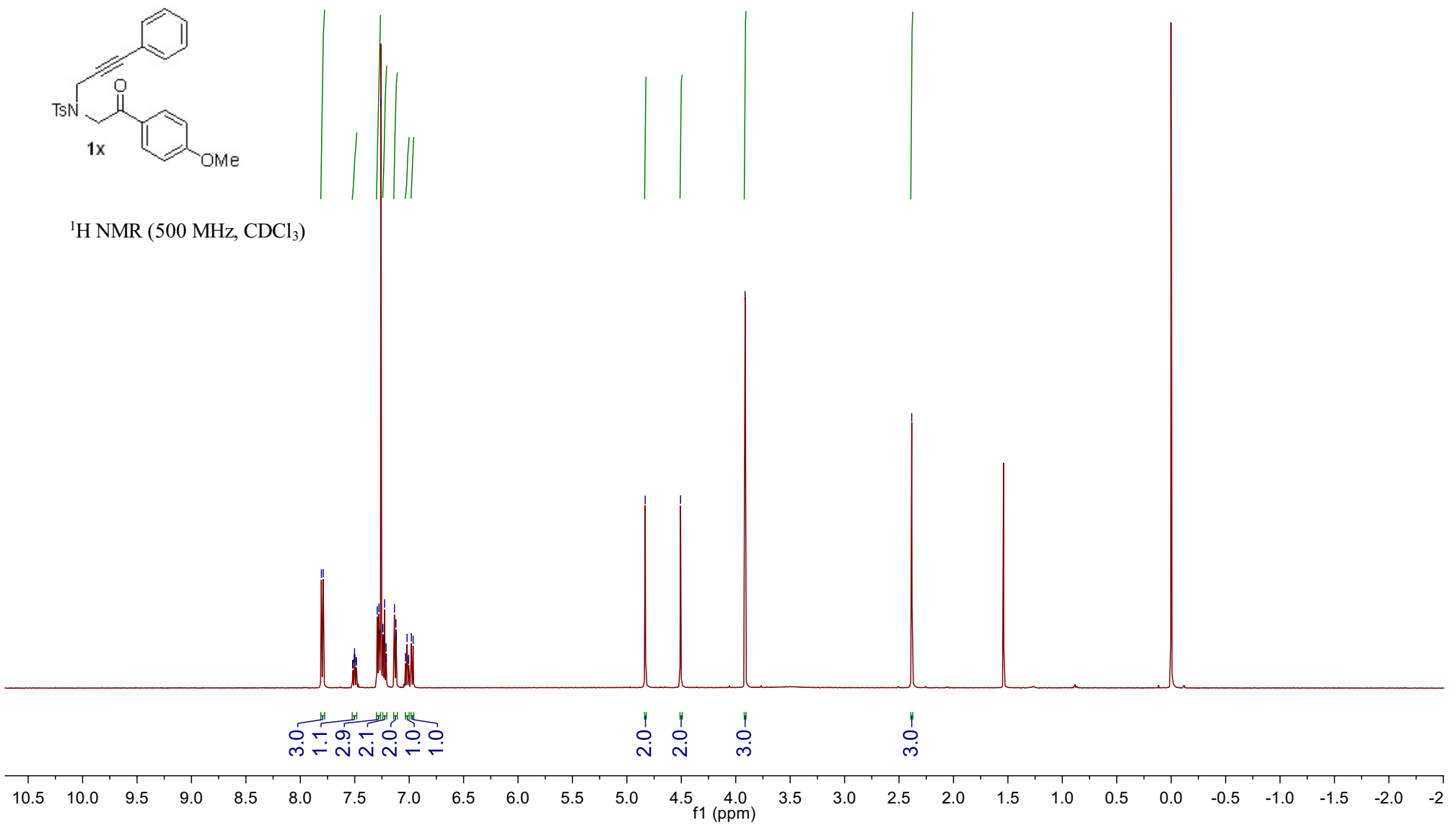




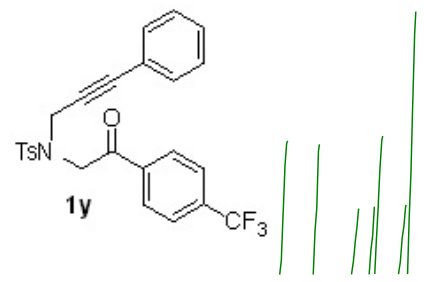

${ }^{1} \mathrm{H} \mathrm{NMR}\left(500 \mathrm{MHz}, \mathrm{CDCl}_{3}\right)$

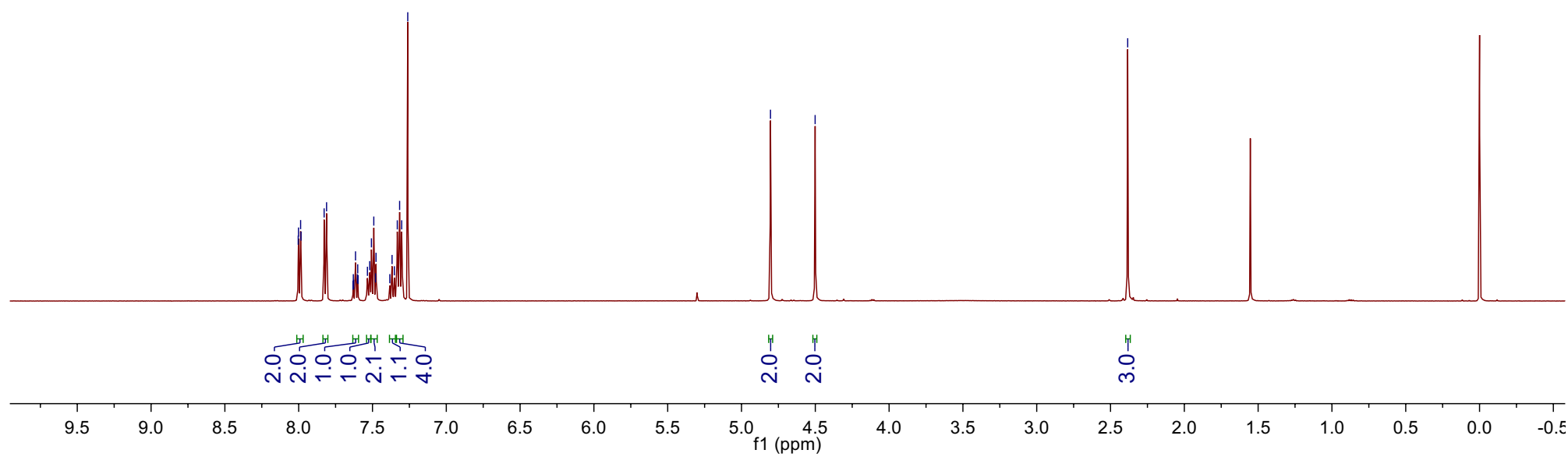




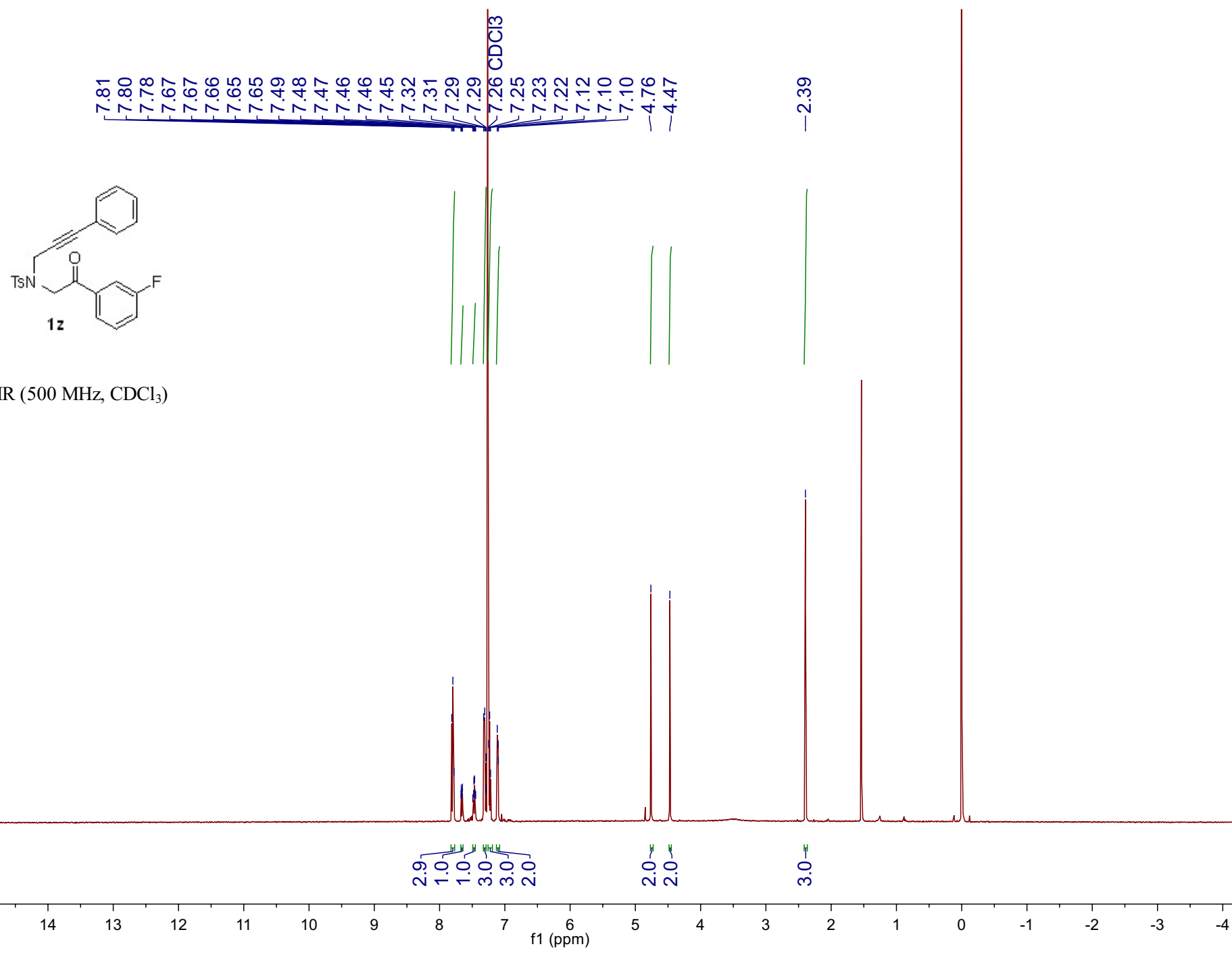


্ֻ

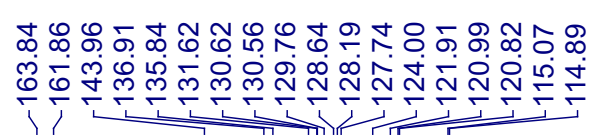

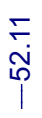

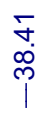

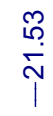<smiles>CC(C)CCC#Cc1ccccc1</smiles>

${ }^{13} \mathrm{C}$ NMR (126 MHz, $\left.\mathrm{CDCl}_{3}\right)$

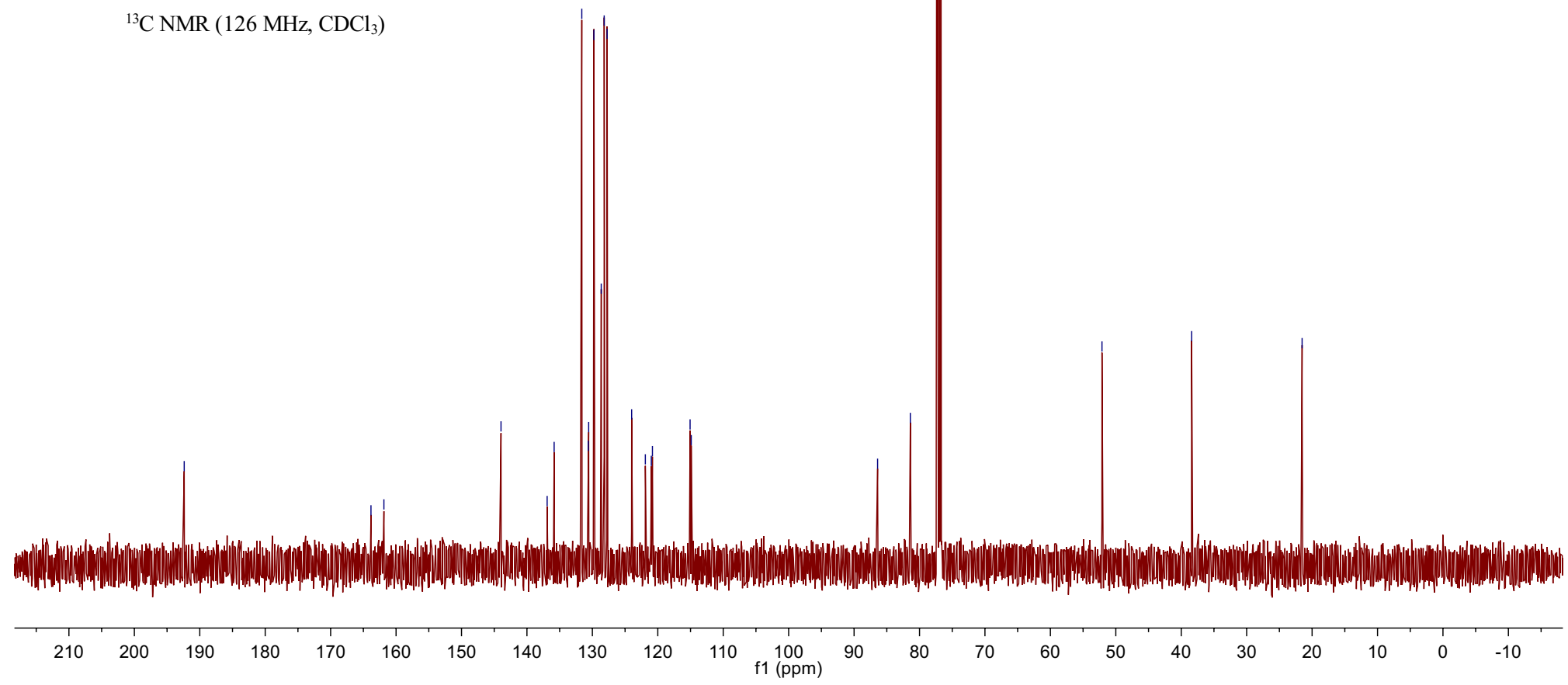


హ゙

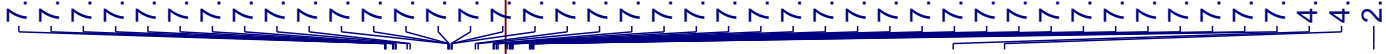
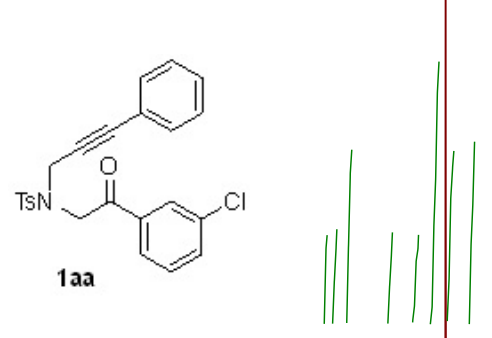

${ }^{1} \mathrm{H} \mathrm{NMR}\left(500 \mathrm{MHz}, \mathrm{CDCl}_{3}\right)$

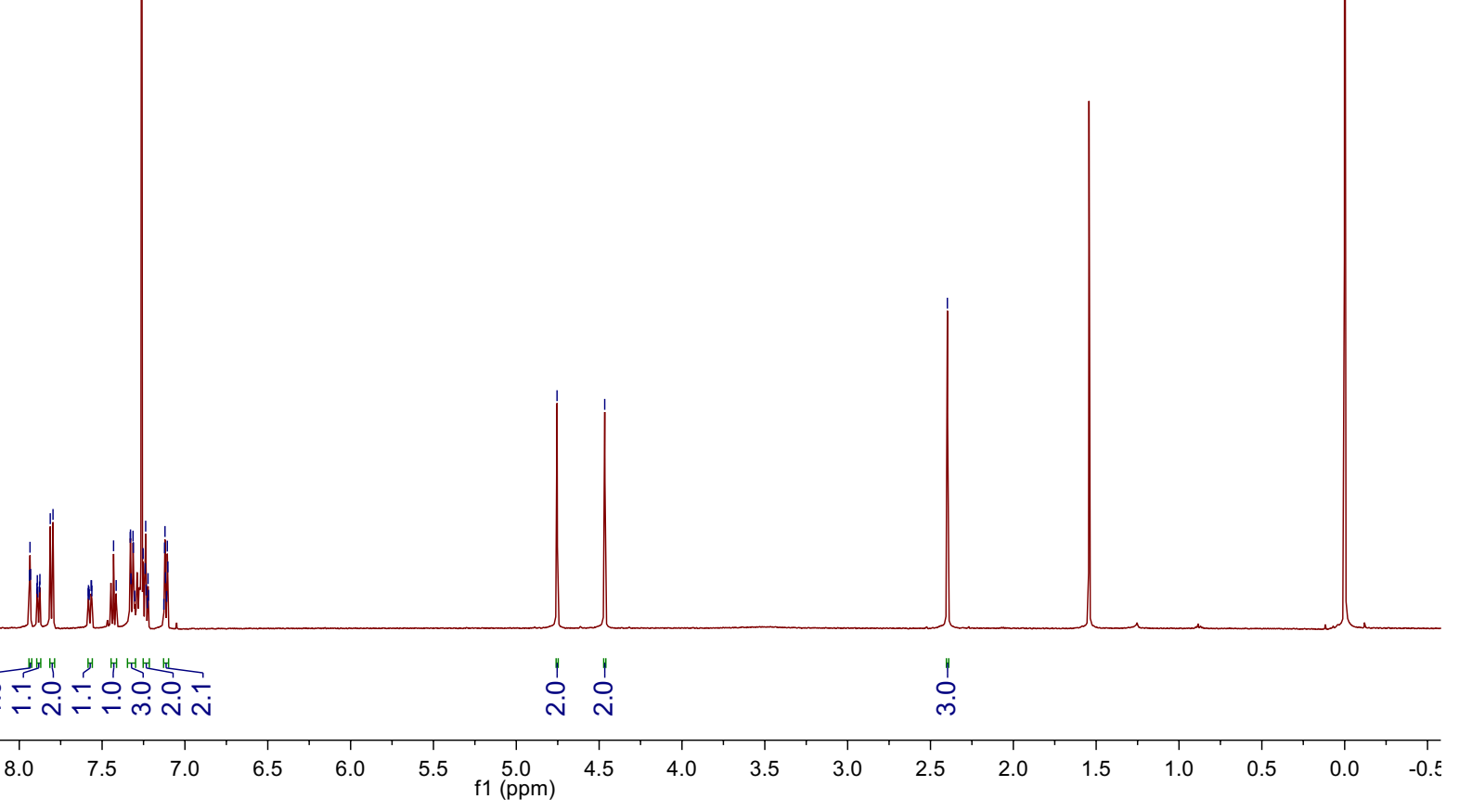




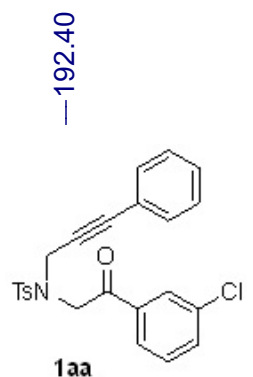

1 aa

${ }^{13} \mathrm{C}$ NMR $\left(126 \mathrm{MHz}, \mathrm{CDCl}_{3}\right.$ )

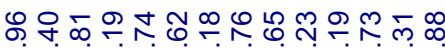

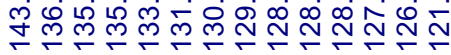

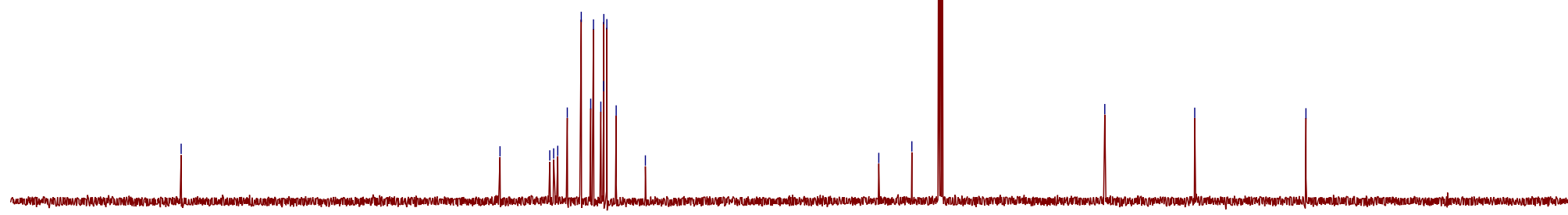



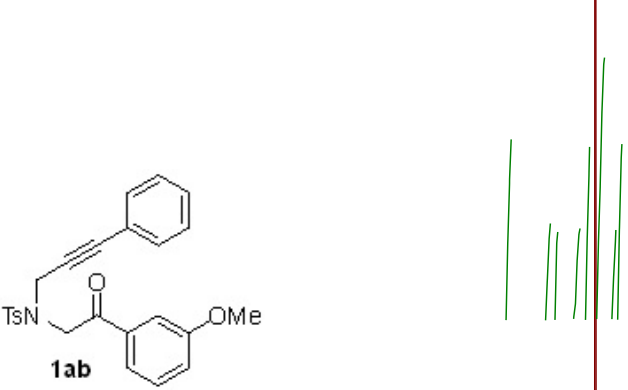

${ }^{1} \mathrm{H} \mathrm{NMR}\left(500 \mathrm{MHz}, \mathrm{CDCl}_{3}\right)$

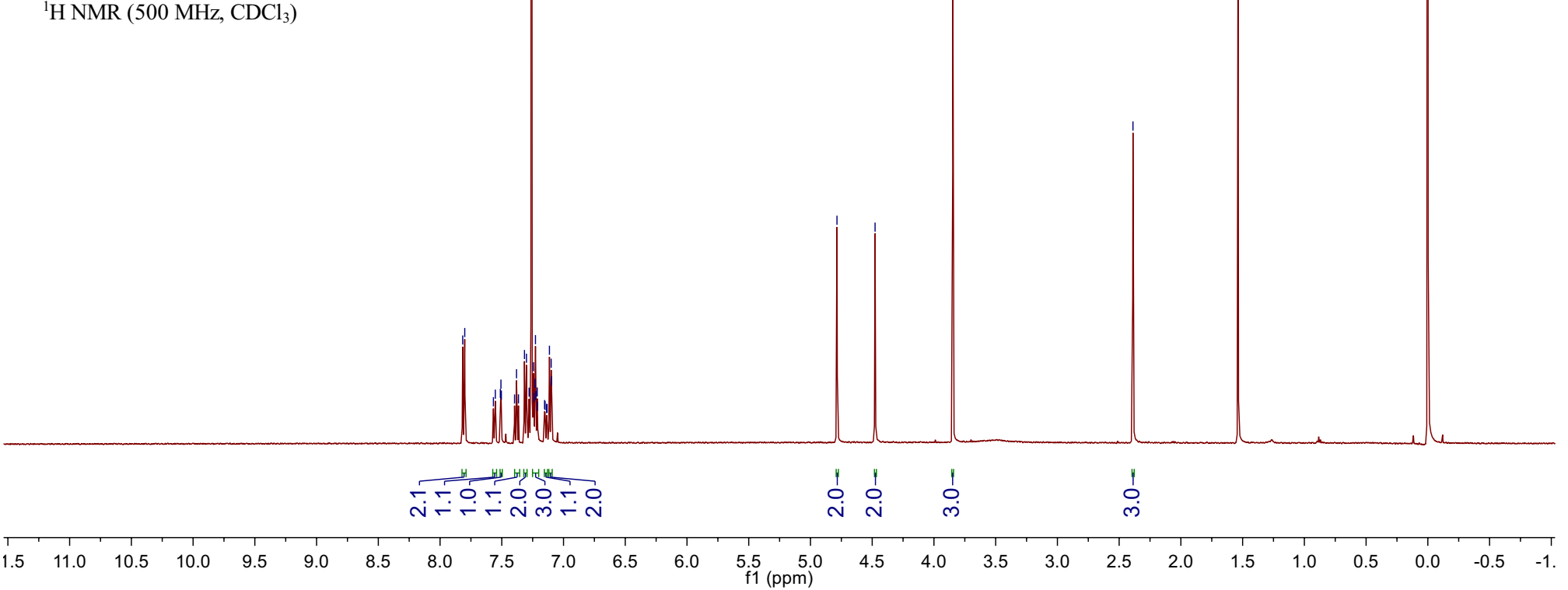




\section{$\frac{m}{0}$}

雨

$\infty$

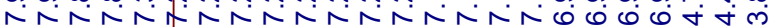
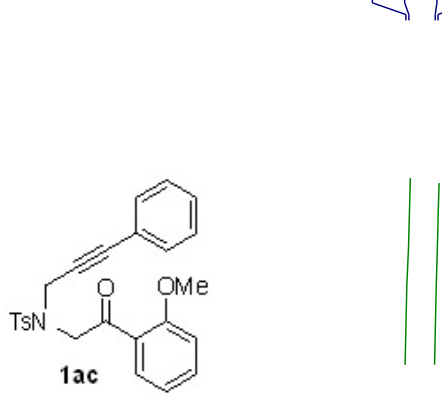

${ }^{1} \mathrm{H} \mathrm{NMR}\left(500 \mathrm{MHz}, \mathrm{CDCl}_{3}\right)$

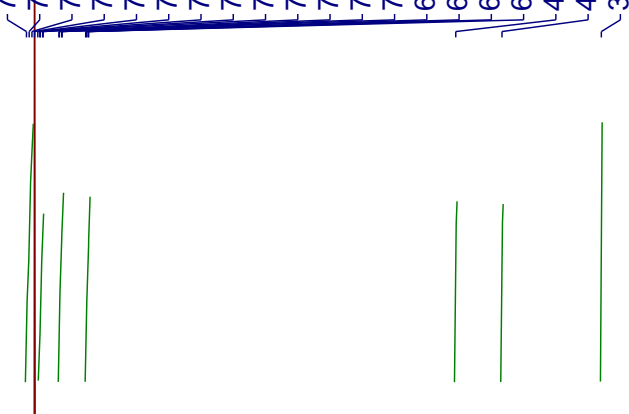

$$
\mid
$$

\begin{tabular}{|c|c|}
\hline 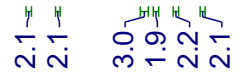 & $\frac{1}{i} \frac{1}{N}$ \\
\hline
\end{tabular}

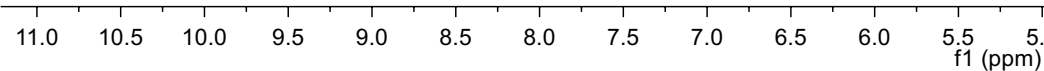



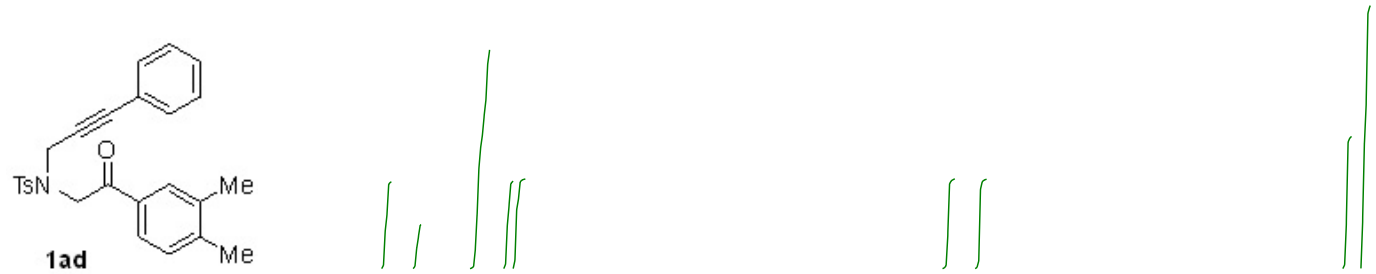

${ }^{1} \mathrm{H}$ NMR (500 MHz, $\left.\mathrm{CDCl}_{3}\right)$

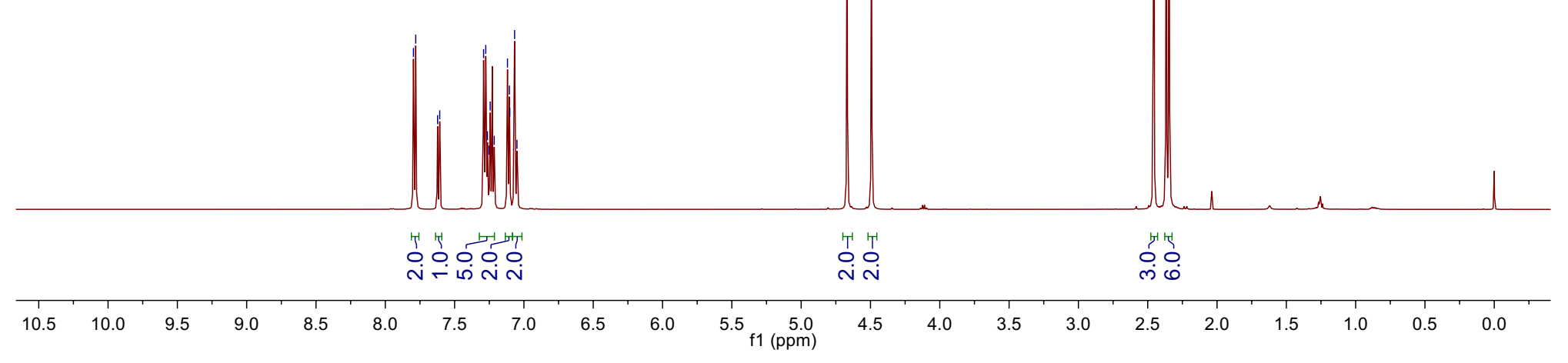


$\bar{m}$ 낭 앙 in

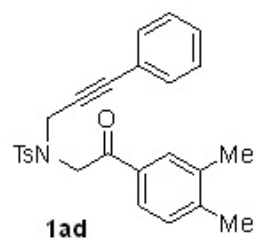

${ }^{13} \mathrm{C}$ NMR (126 MHz, $\mathrm{CDCl}_{3}$ )

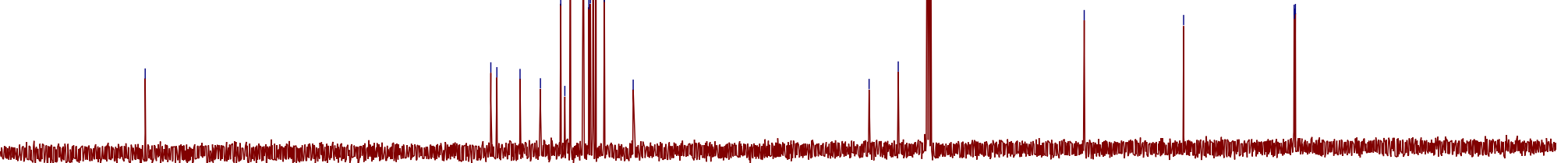

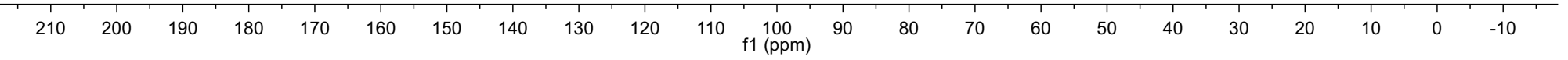




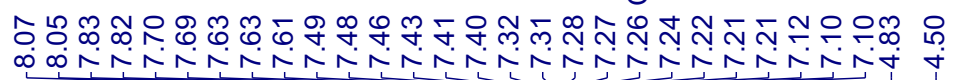

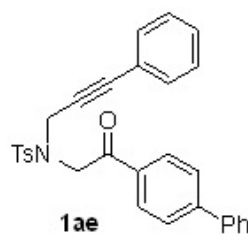

${ }^{1} \mathrm{H}$ NMR (500 MHz, $\mathrm{CDCl}_{3}$ )
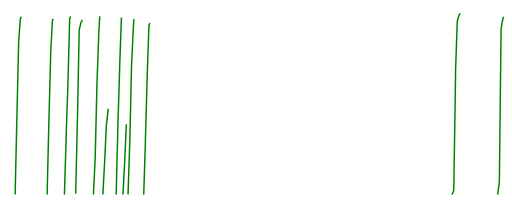

$\stackrel{\substack{m \\ i}}{i}$

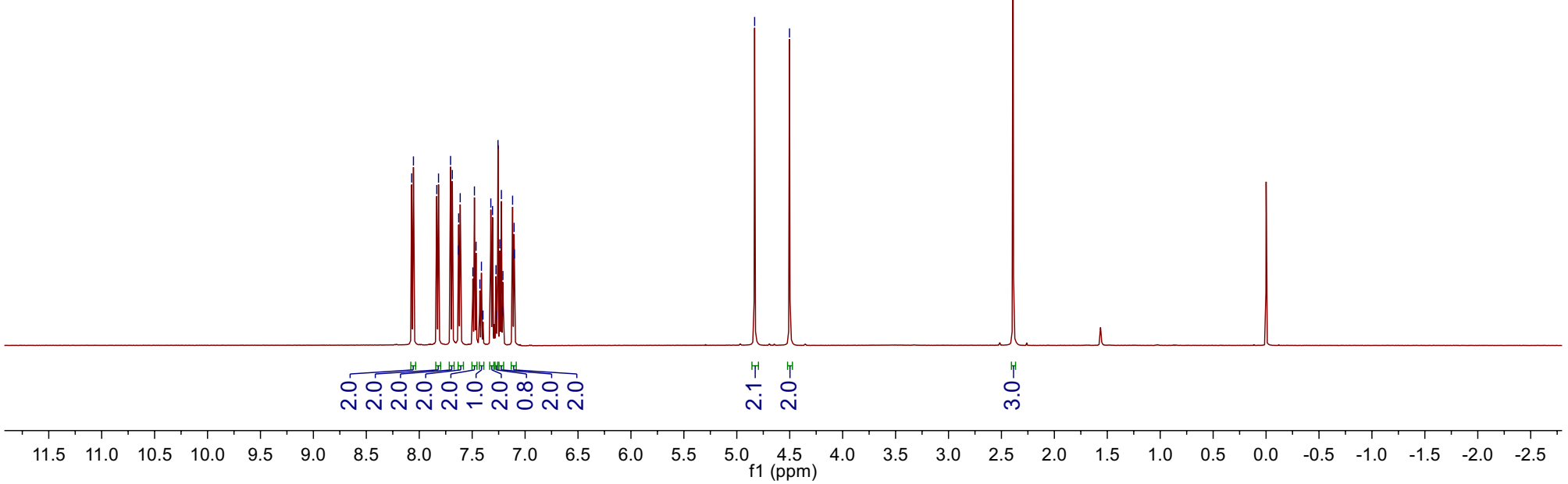




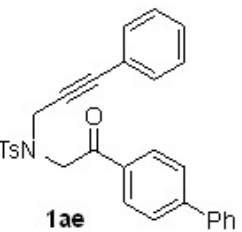

${ }^{13} \mathrm{C} \mathrm{NMR}\left(126 \mathrm{MHz}, \mathrm{CDCl}_{3}\right)$

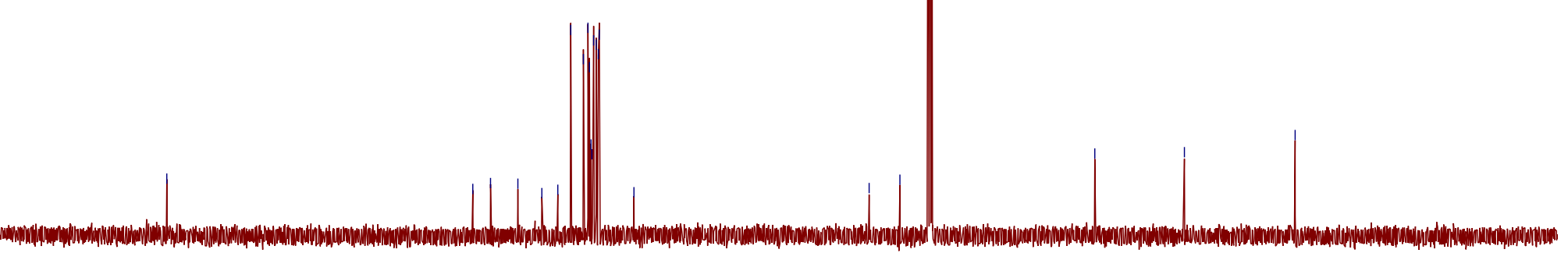




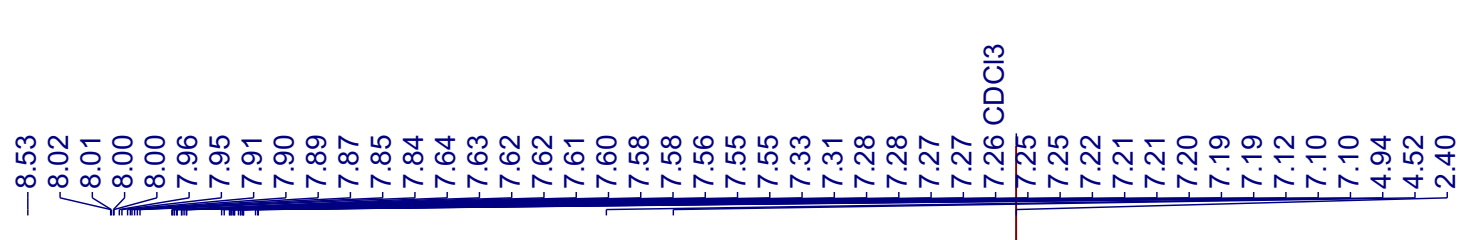
ind 


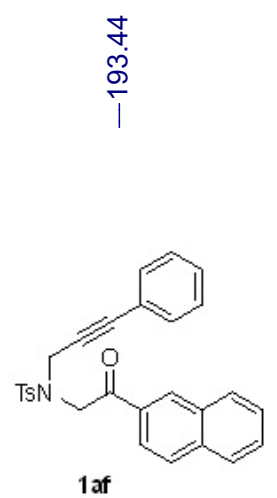

${ }^{13} \mathrm{C}$ NMR (126 MHz, $\mathrm{CDCl}_{3}$ )

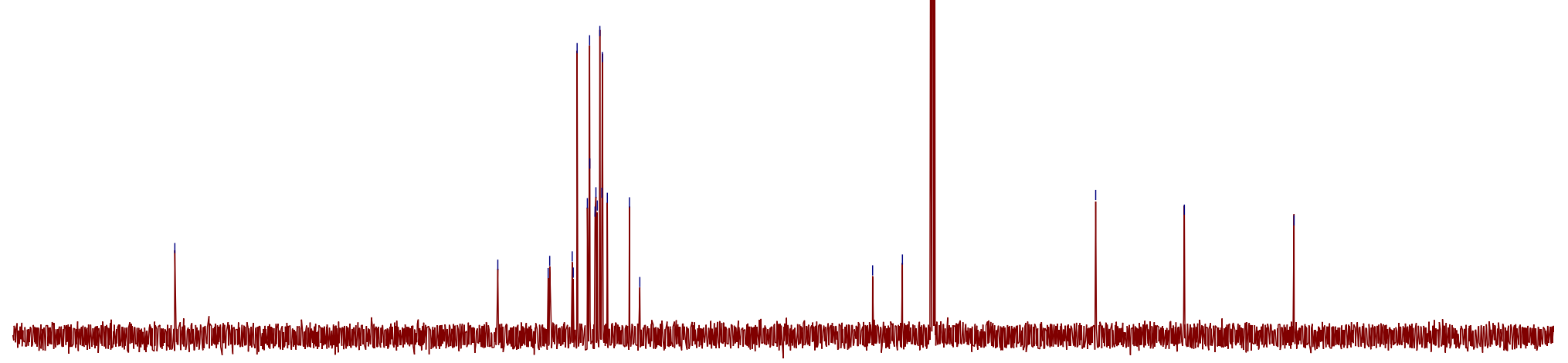


ํㅡㄴ

ヘト゚ำำำษ

NNNNNN

过

ก)

$\stackrel{\infty}{\leftarrow}$
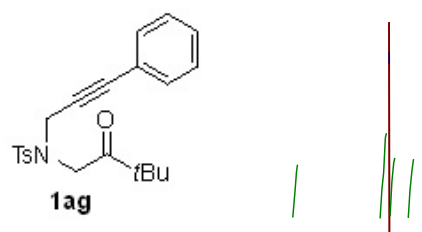

${ }^{1} \mathrm{H} \mathrm{NMR}\left(500 \mathrm{MHz}, \mathrm{CDCl}_{3}\right)$
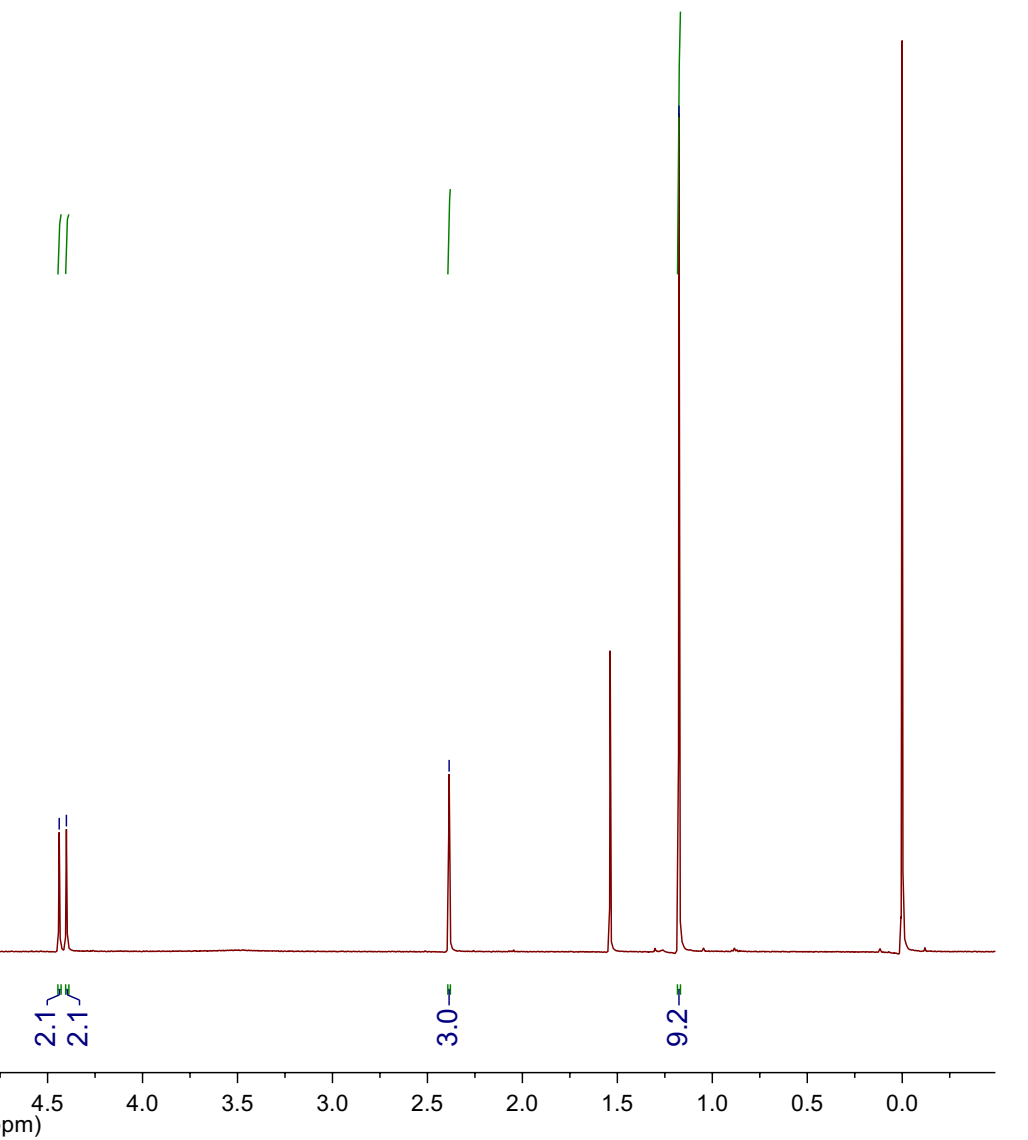


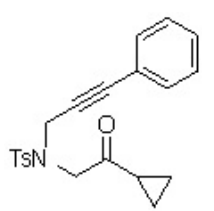

1 ah
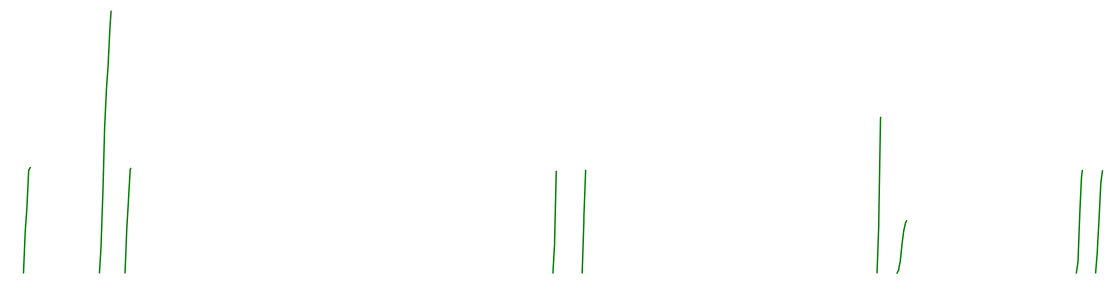

${ }^{1} \mathrm{H} \mathrm{NMR}\left(500 \mathrm{MHz}, \mathrm{CDCl}_{3}\right)$

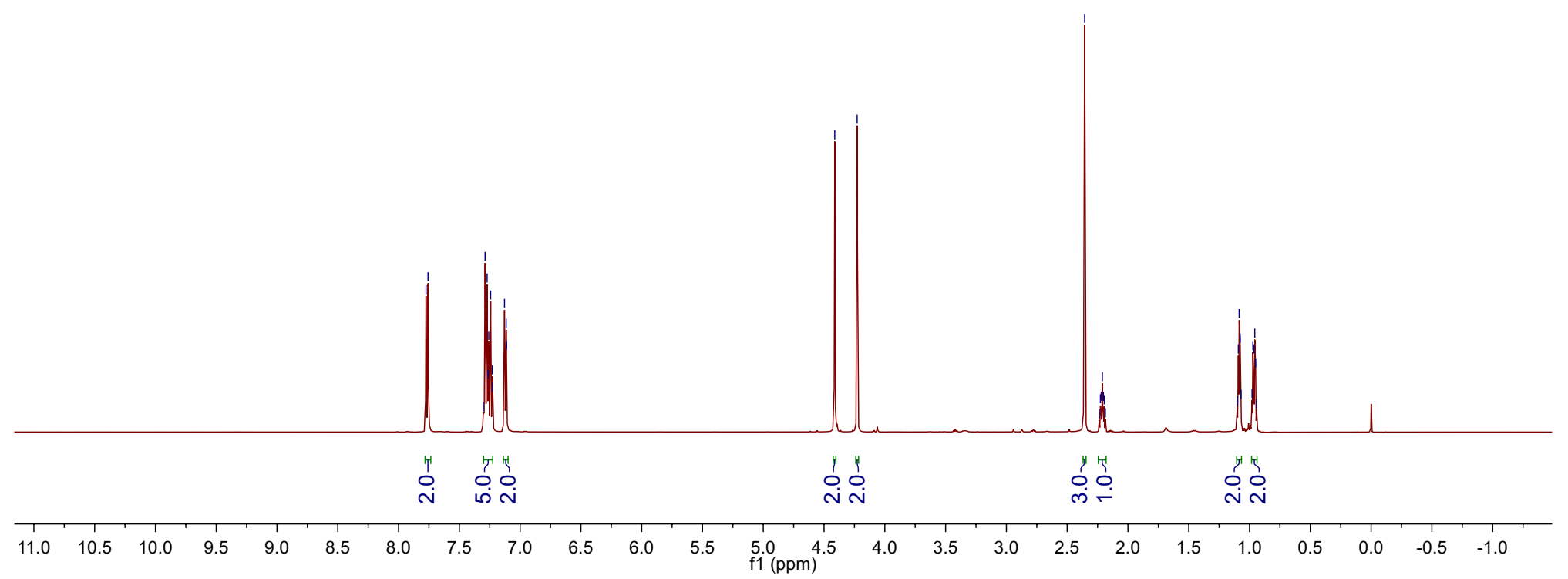




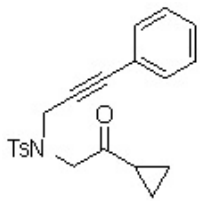

1 ah

${ }^{13} \mathrm{C}$ NMR $\left(126 \mathrm{MHz}, \mathrm{CDCl}_{3}\right)$

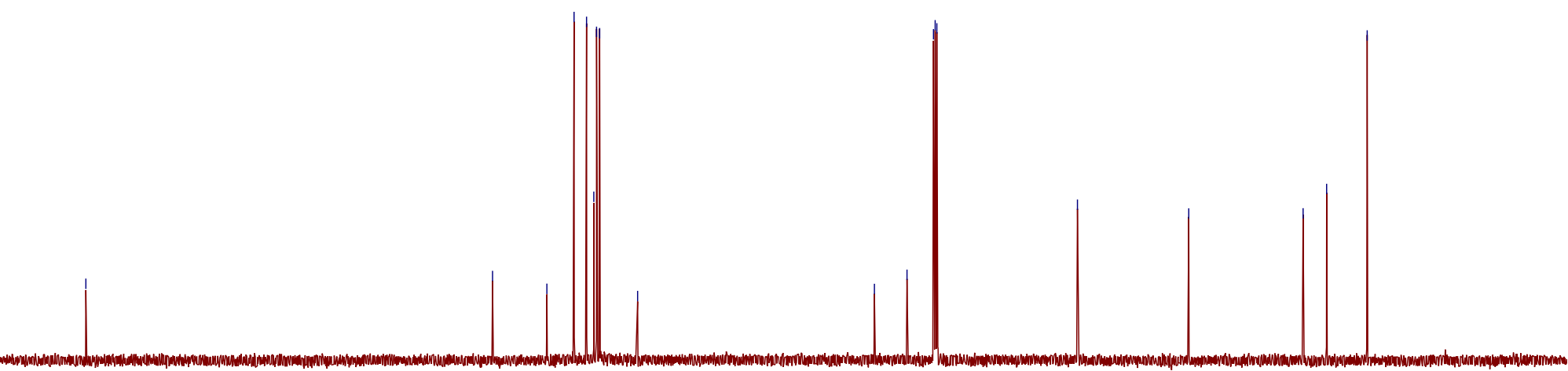

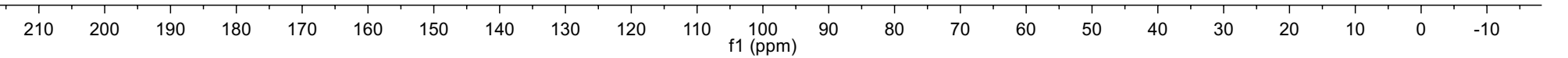


ํㅡㄴ

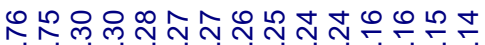

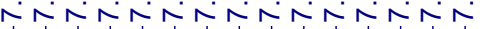

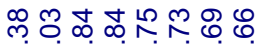

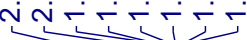
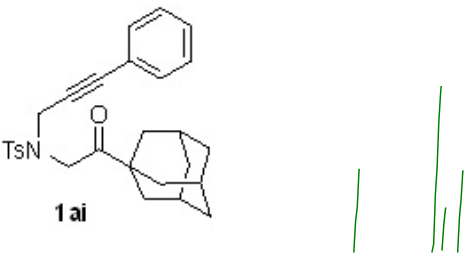

${ }^{1} \mathrm{H} \mathrm{NMR}\left(500 \mathrm{MHz}, \mathrm{CDCl}_{3}\right)$

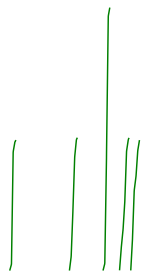

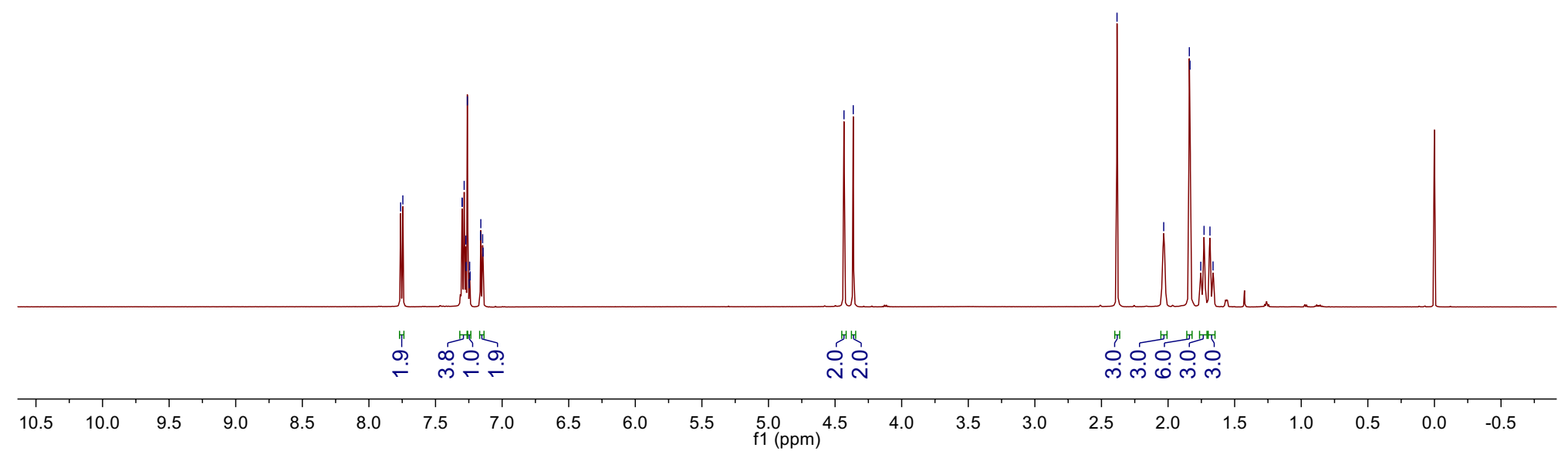




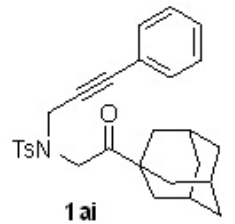

${ }^{13} \mathrm{C}$ NMR $\left(126 \mathrm{MHz}, \mathrm{CDCl}_{3}\right)$

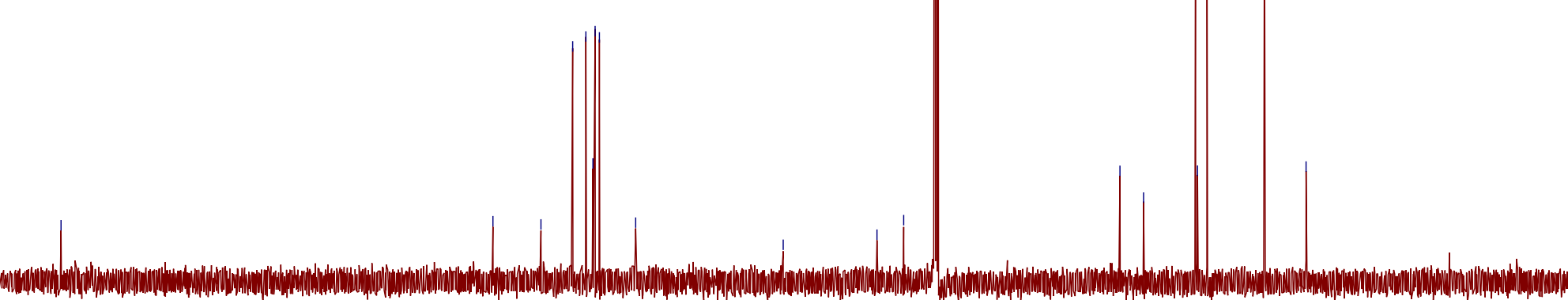




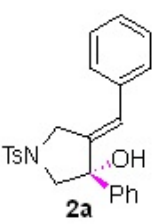

${ }^{1} \mathrm{H}$ NMR (500 MHz, $\left.\mathrm{CDCl}_{3}\right)$
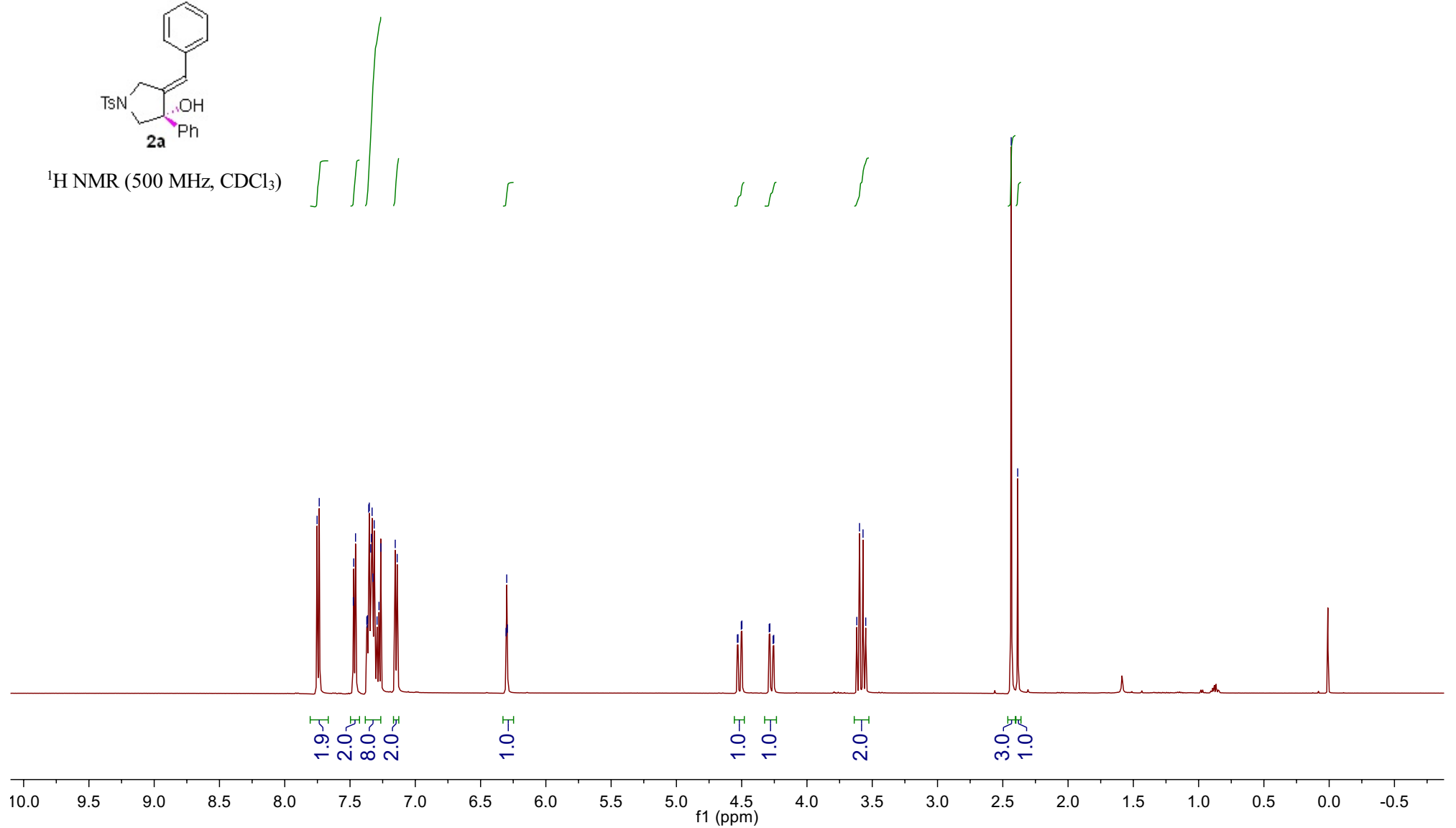

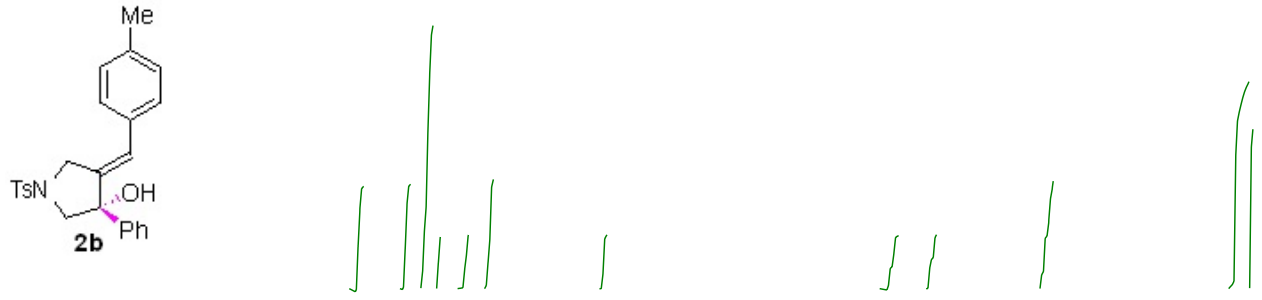

${ }^{1} \mathrm{H} \mathrm{NMR}\left(500 \mathrm{MHz}, \mathrm{CDCl}_{3}\right)$

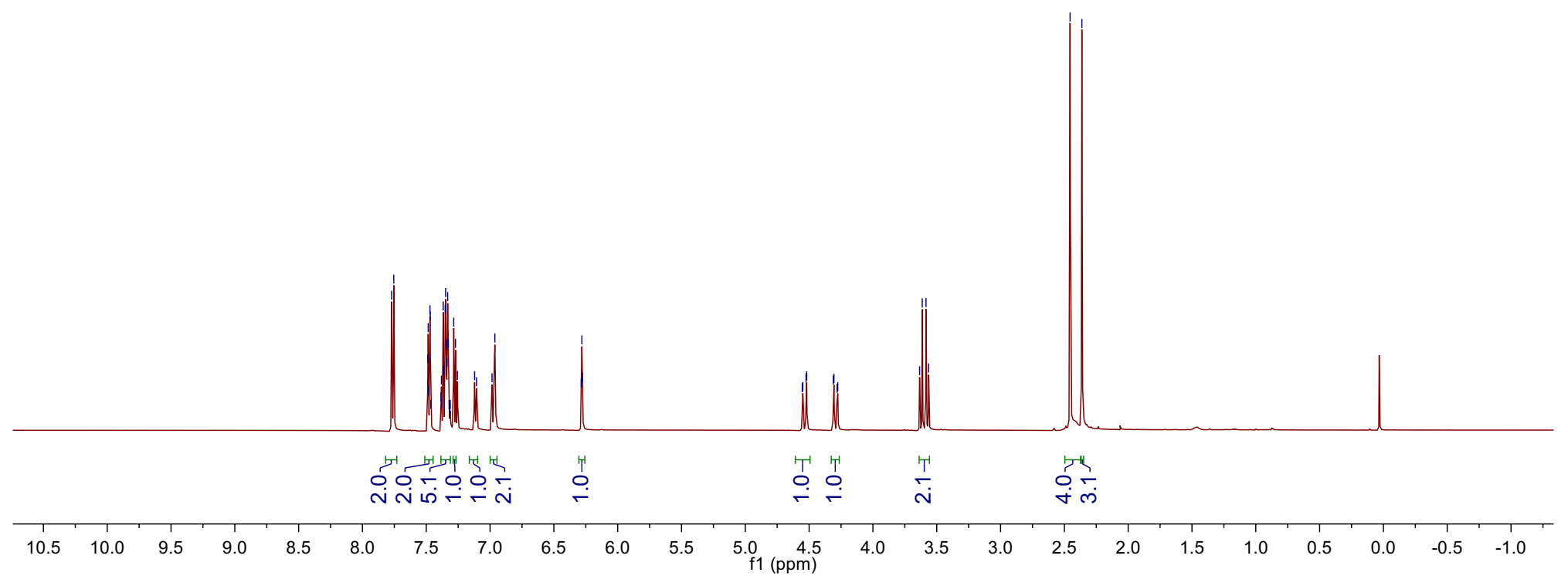




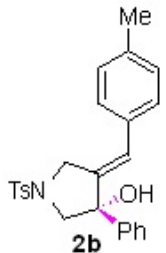

${ }^{13} \mathrm{C} \mathrm{NMR}\left(126 \mathrm{MHz}, \mathrm{CDCl}_{3}\right)$
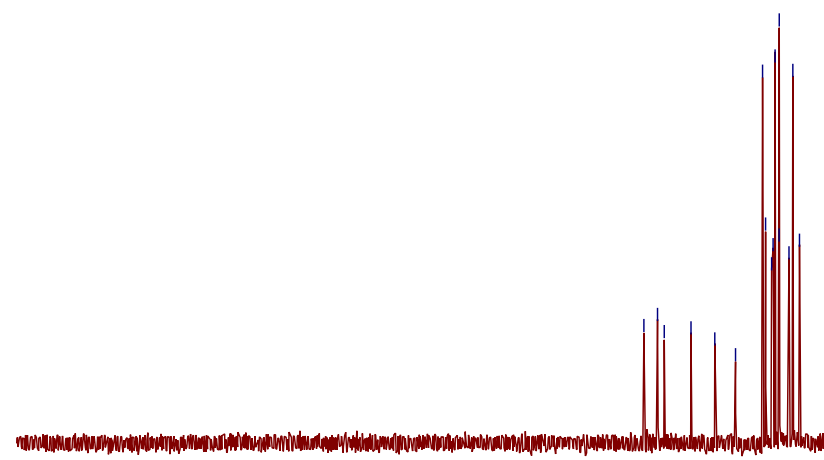

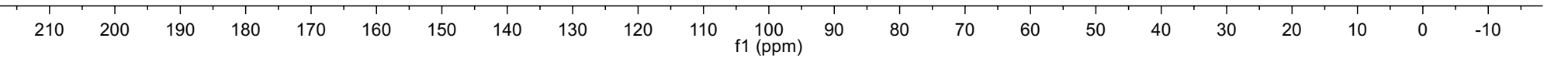




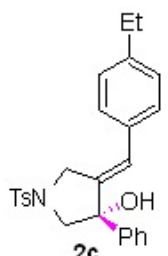

${ }^{1} \mathrm{H} \mathrm{NMR}\left(500 \mathrm{MHz}, \mathrm{CDCl}_{3}\right)$

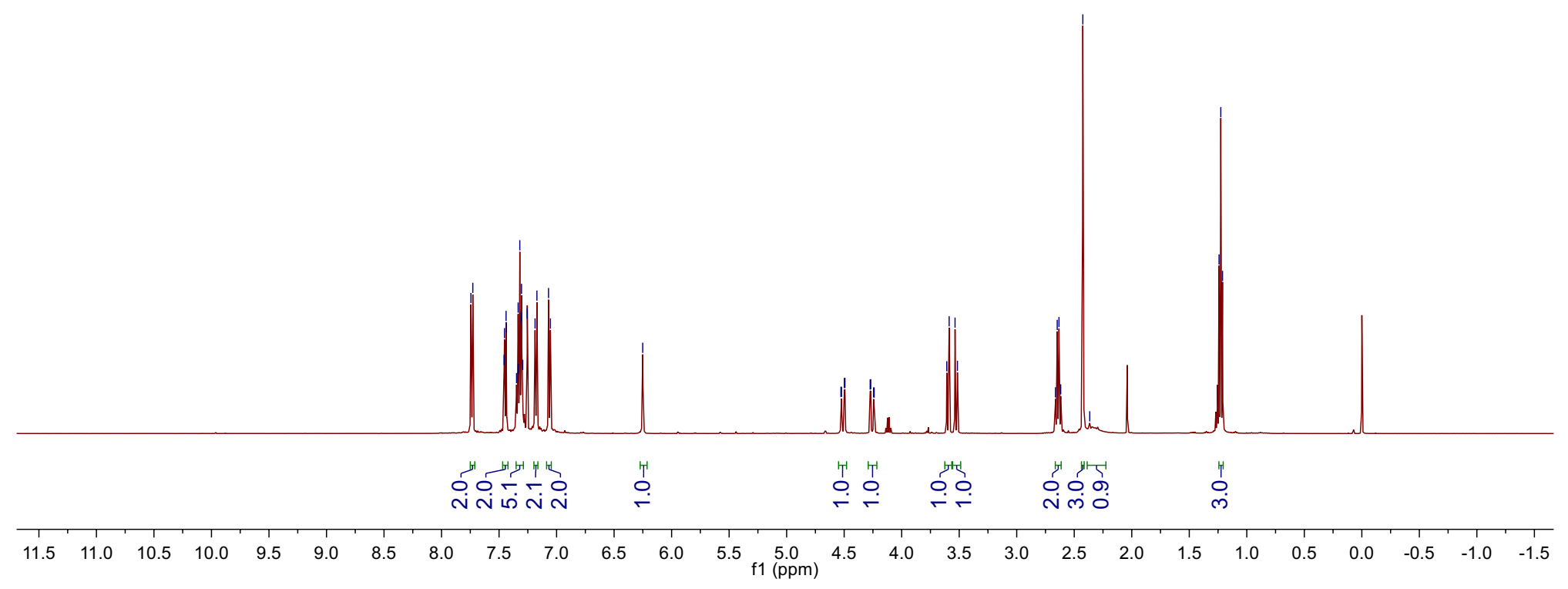




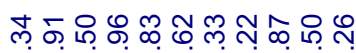

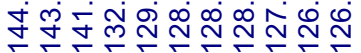

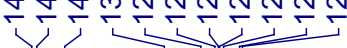

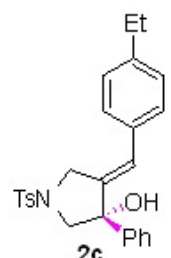

${ }^{13} \mathrm{C}$ NMR $\left(126 \mathrm{MHz}, \mathrm{CDCl}_{3}\right)$
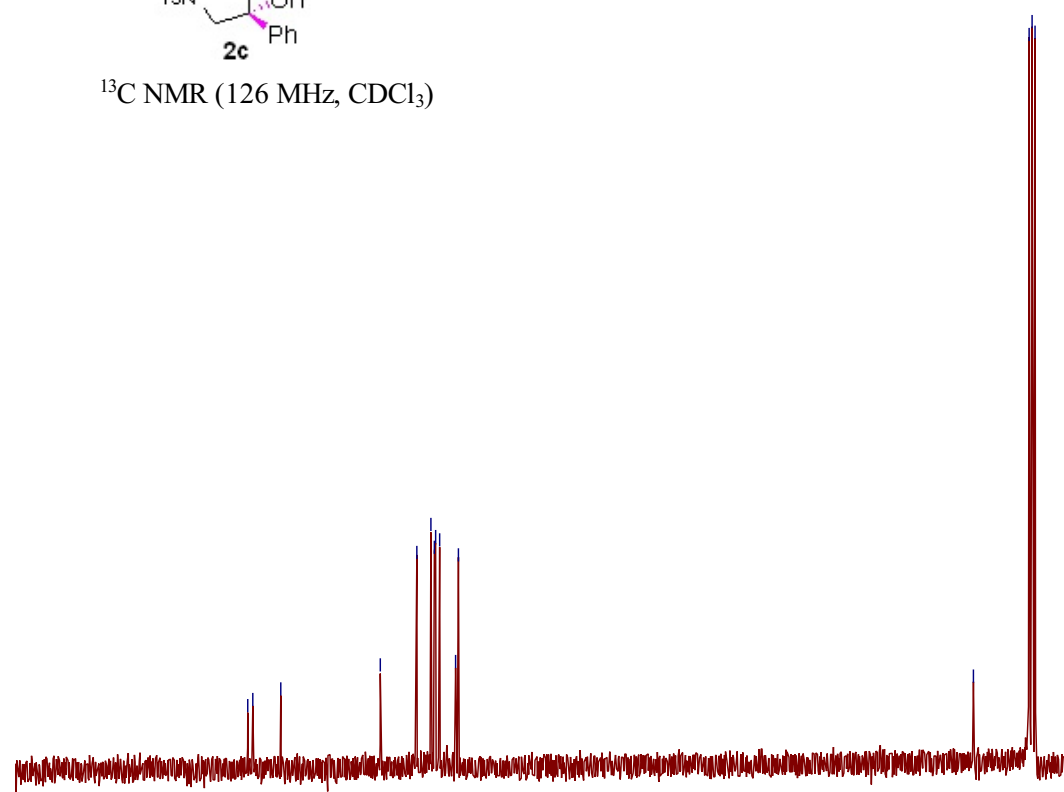

120

110

100

90 
응

م

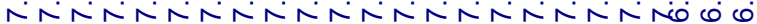

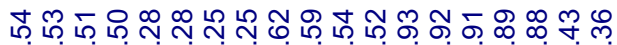
$\forall \forall \forall \forall \forall \forall \forall \forall$ m

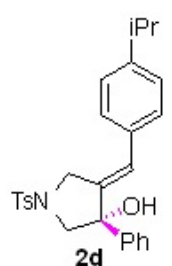

${ }^{1} \mathrm{H} \mathrm{NMR}\left(500 \mathrm{MHz}, \mathrm{CDCl}_{3}\right)$
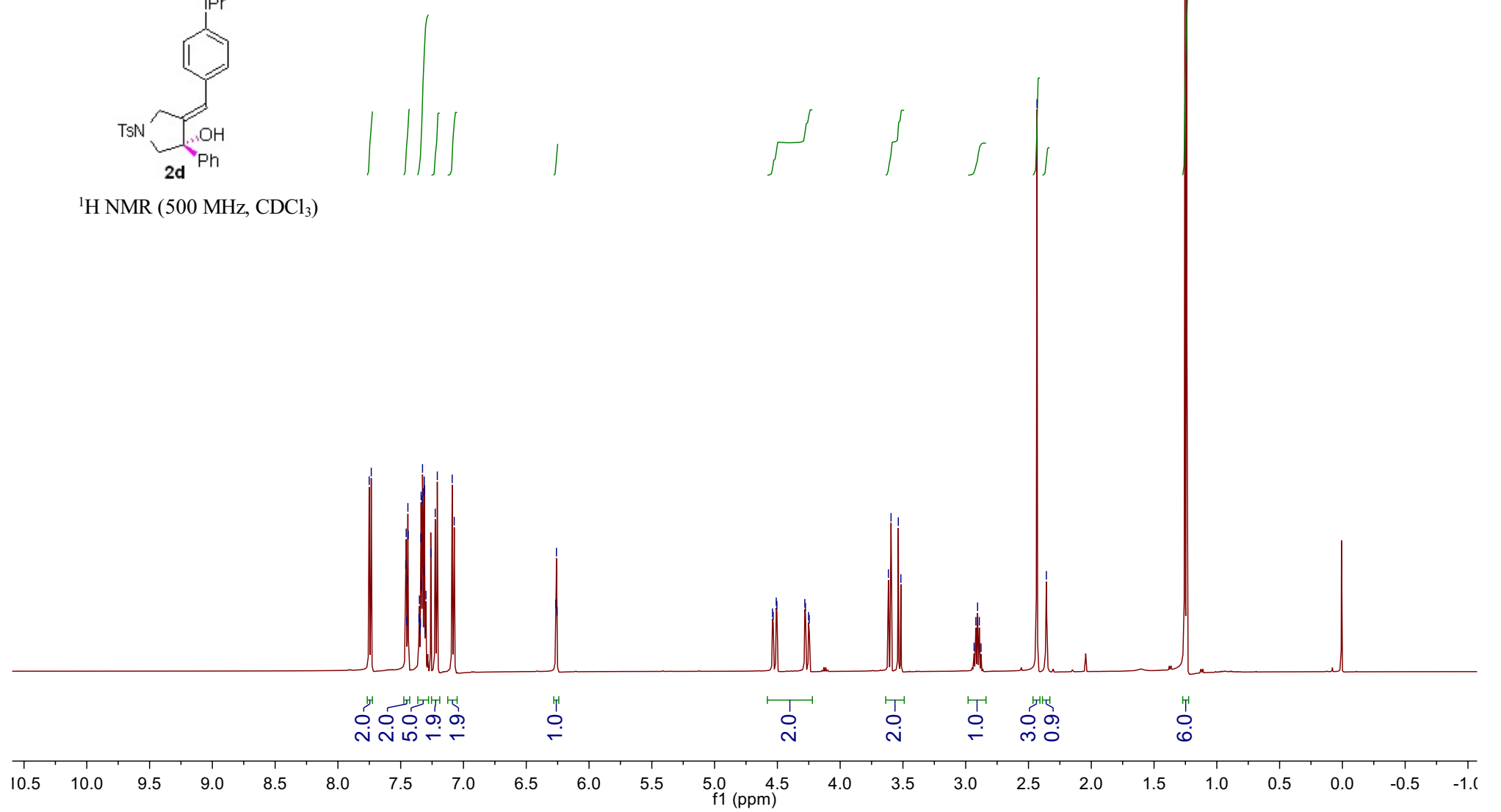


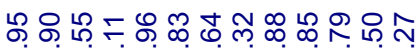

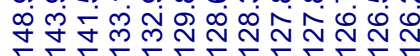

ᄂ

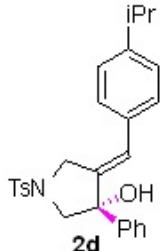

$\left.{ }^{13} \mathrm{C} \mathrm{NMR} \mathrm{(126} \mathrm{MHz,} \mathrm{CDCl}_{3}\right)$

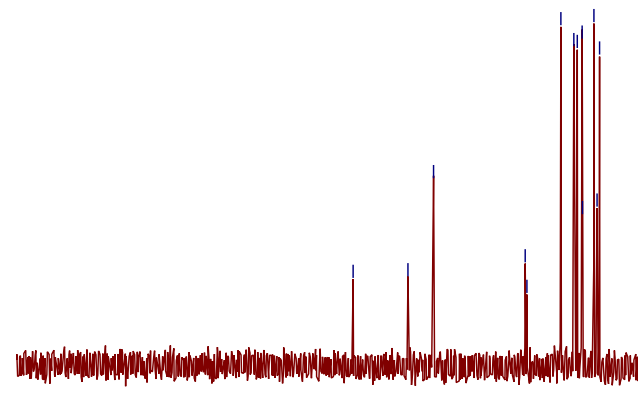



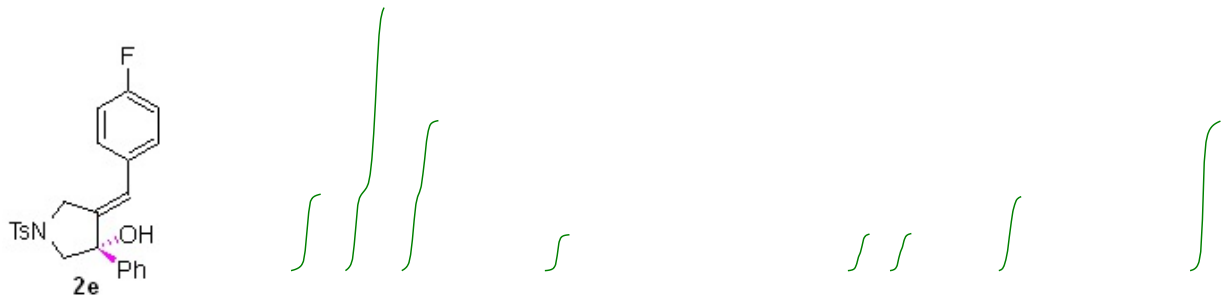

${ }^{1} \mathrm{H} \mathrm{NMR}\left(500 \mathrm{MHz}, \mathrm{CDCl}_{3}\right)$

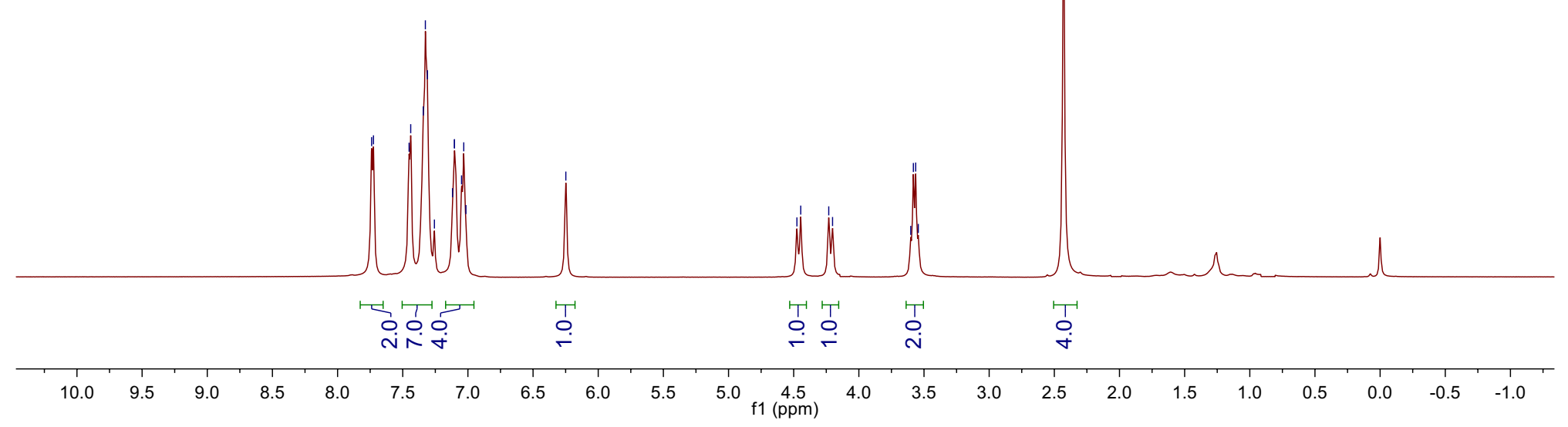


ธำฺ

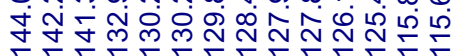
先

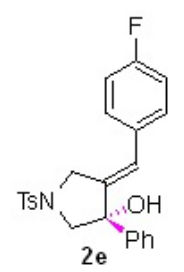

${ }^{13} \mathrm{C}$ NMR (126 MHz, $\left.\mathrm{CDCl}_{3}\right)$ $m \frac{m}{0}$

ชิน

†

六 $N$

$\checkmark$ i

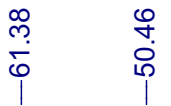
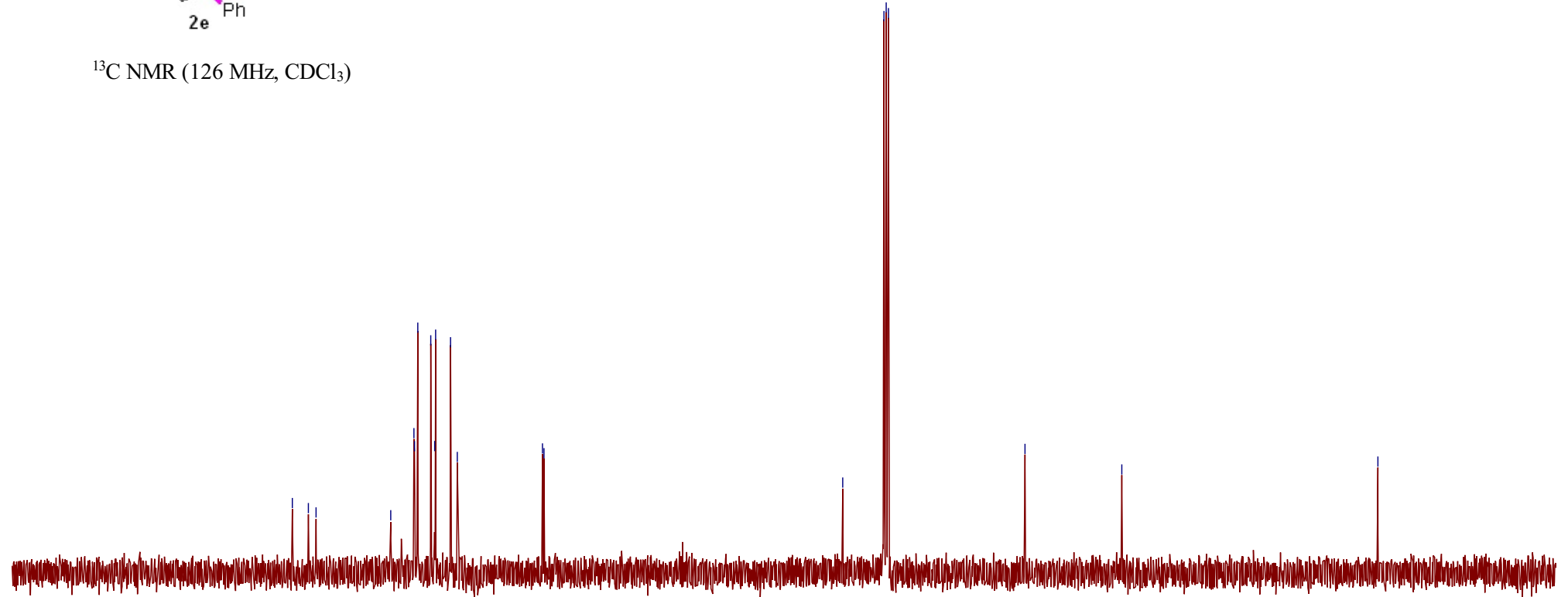

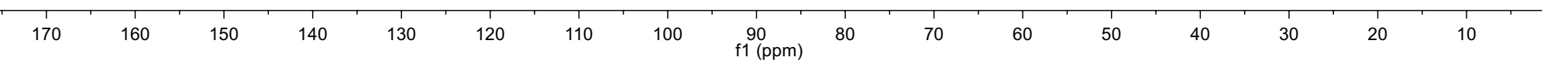



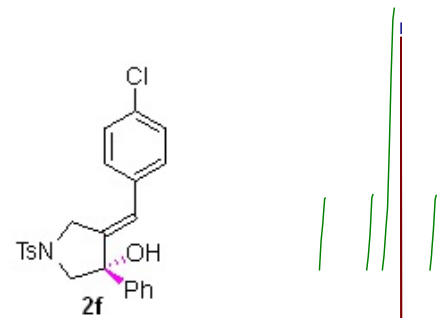

${ }^{1} \mathrm{H} \mathrm{NMR}\left(500 \mathrm{MHz}, \mathrm{CDCl}_{3}\right)$

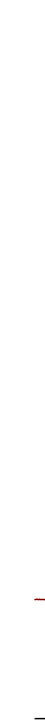




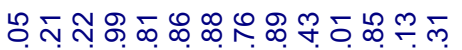

守守守孞

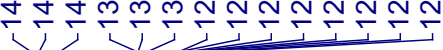

$\stackrel{\infty}{\sim}$ 于

욤

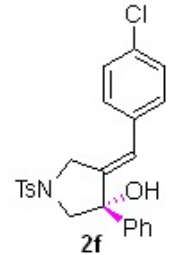

${ }^{13} \mathrm{C}$ NMR (126 MHz, $\mathrm{CDCl}_{3}$ )

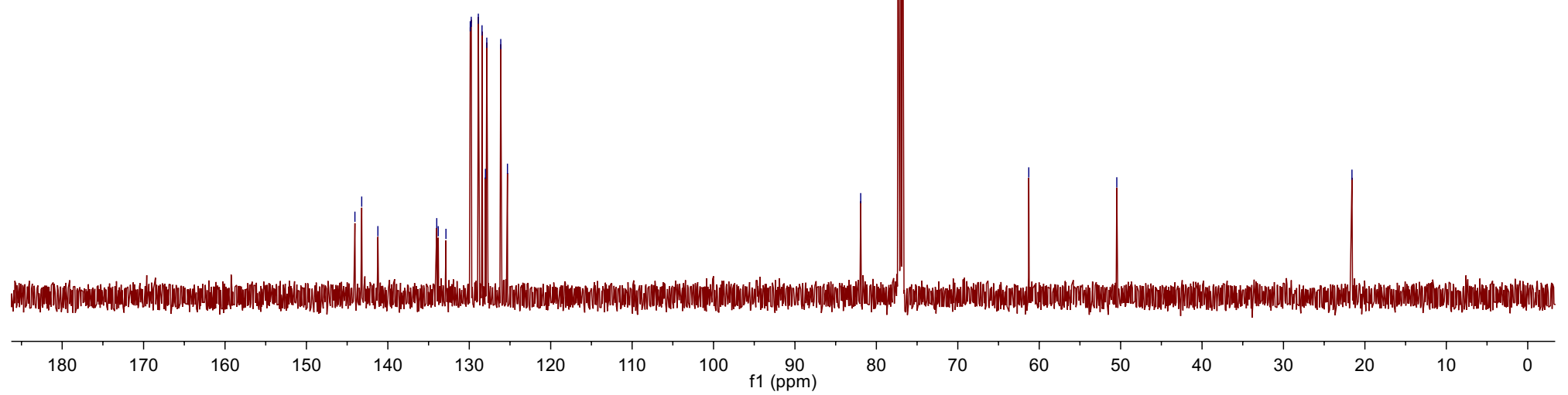


夏

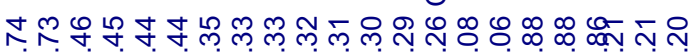

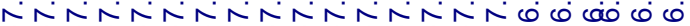

${ }^{1} \mathrm{H}$ NMR (500 MHz, $\mathrm{CDCl}_{3}$ )

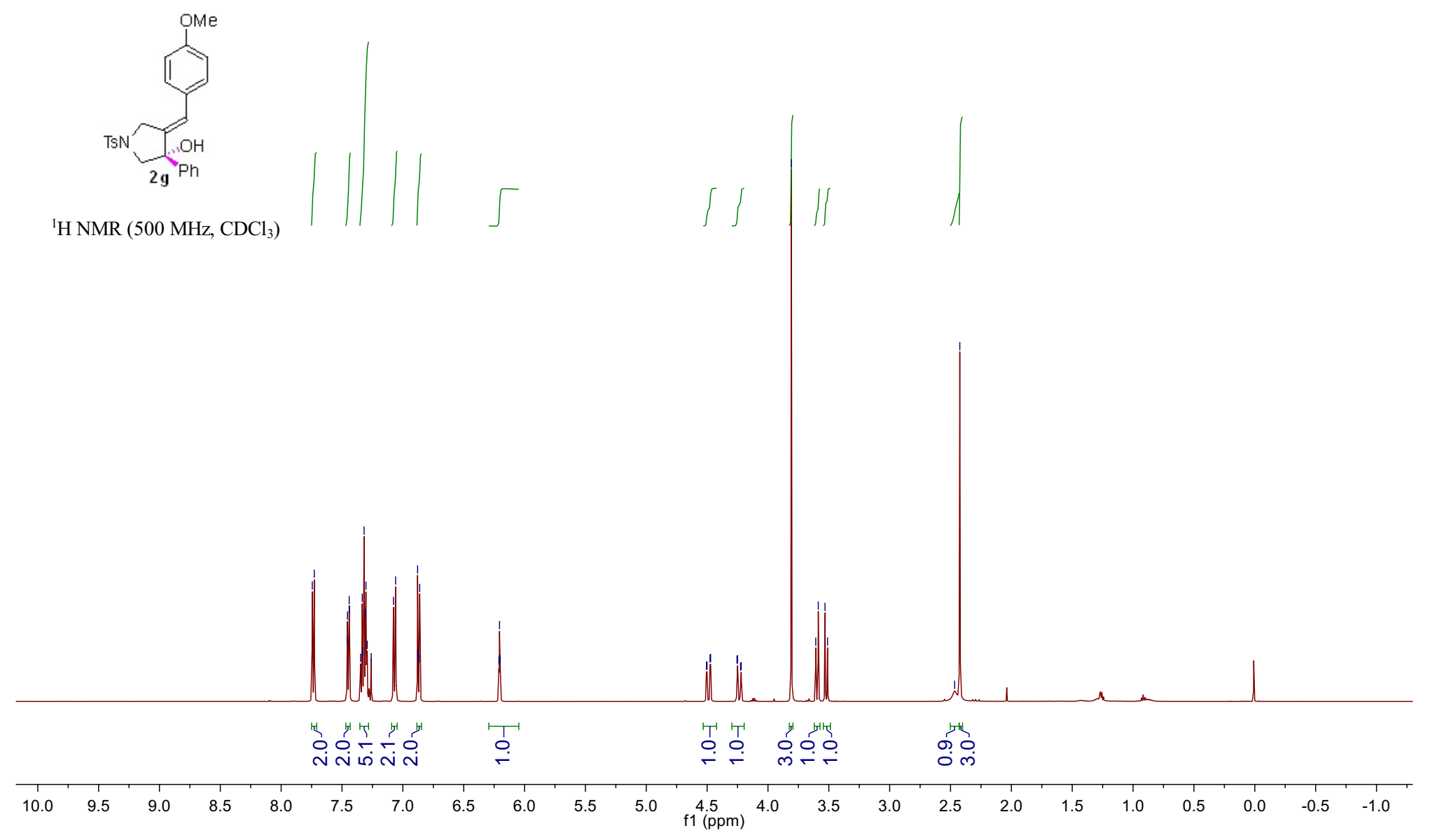

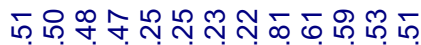

A

ชั่ ขับ

กิ่ 


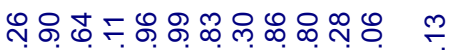

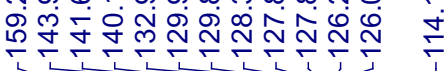

$\frac{m}{0} \frac{m}{0} \frac{m}{0}$

ดู

ㄷํ요

於令
रि

ธำ 요

111 $\stackrel{\infty}{\infty}$

స

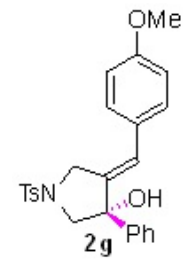

${ }^{13} \mathrm{C} \mathrm{NMR}\left(126 \mathrm{MHz}, \mathrm{CDCl}_{3}\right)$

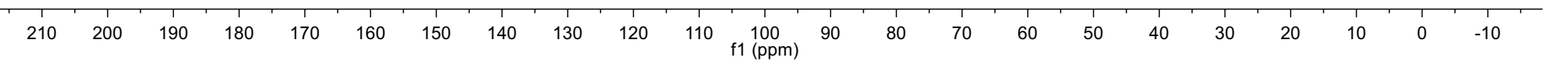


$\frac{m}{0}$

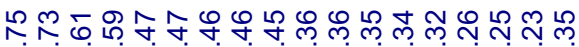

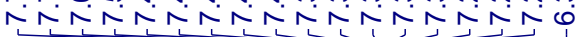

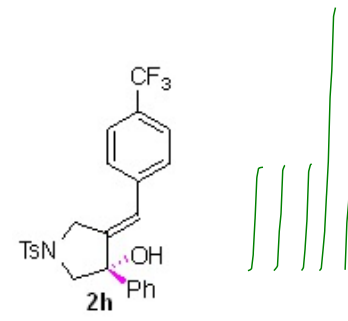

${ }^{1} \mathrm{H} \mathrm{NMR}\left(500 \mathrm{MHz}, \mathrm{CDCl}_{3}\right)$

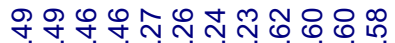

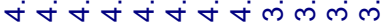

$\stackrel{8}{\mathcal{2}}$

กับ

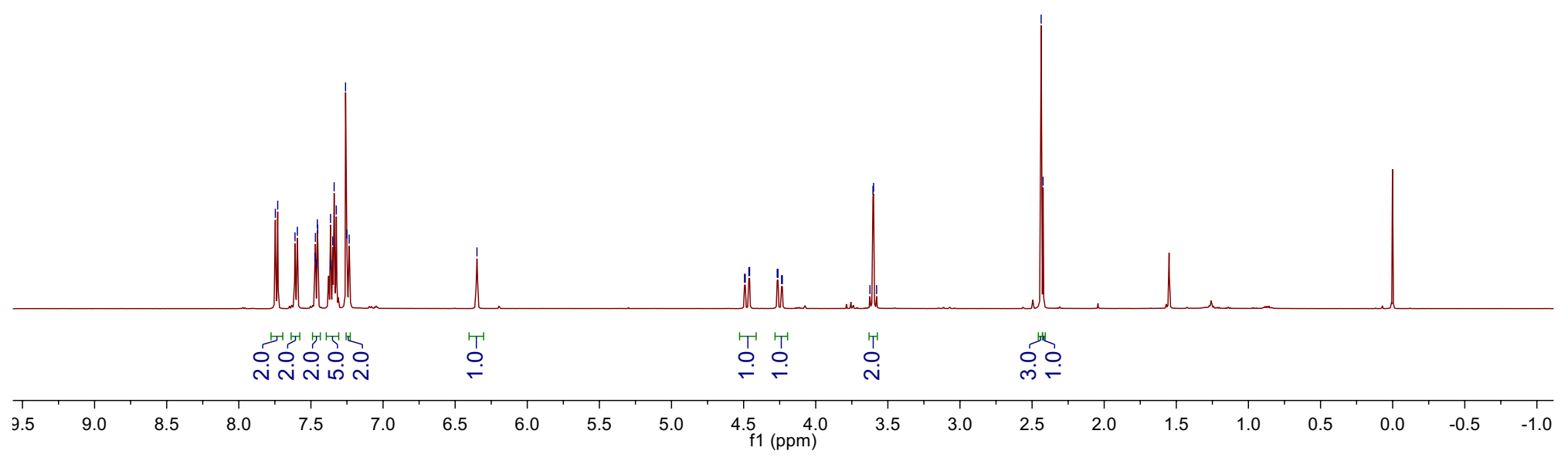




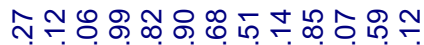
守守守市 $i \frac{1}{1}$

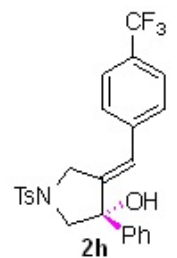

${ }^{13} \mathrm{C}$ NMR $\left(126 \mathrm{MHz}, \mathrm{CDCl}_{3}\right)$

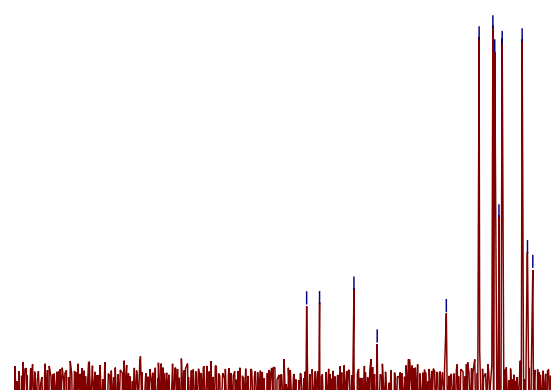



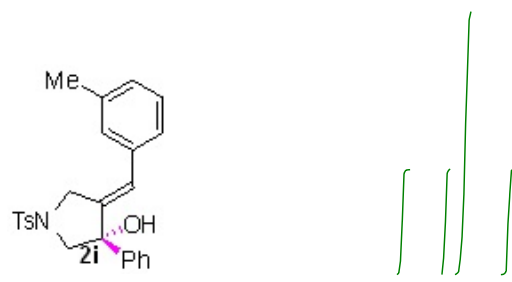

${ }^{1} \mathrm{H} \mathrm{NMR}\left(500 \mathrm{MHz}, \mathrm{CDCl}_{3}\right)$

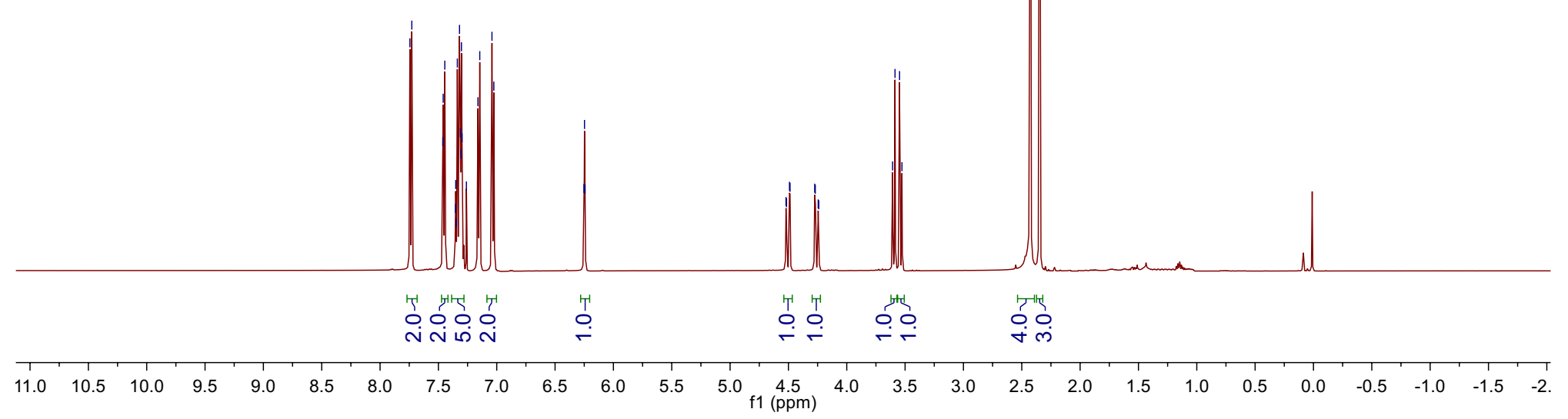




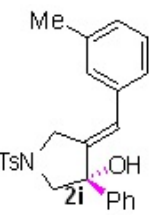

${ }^{13} \mathrm{C}$ NMR (126 MHz, $\left.\mathrm{CDCl}_{3}\right)$
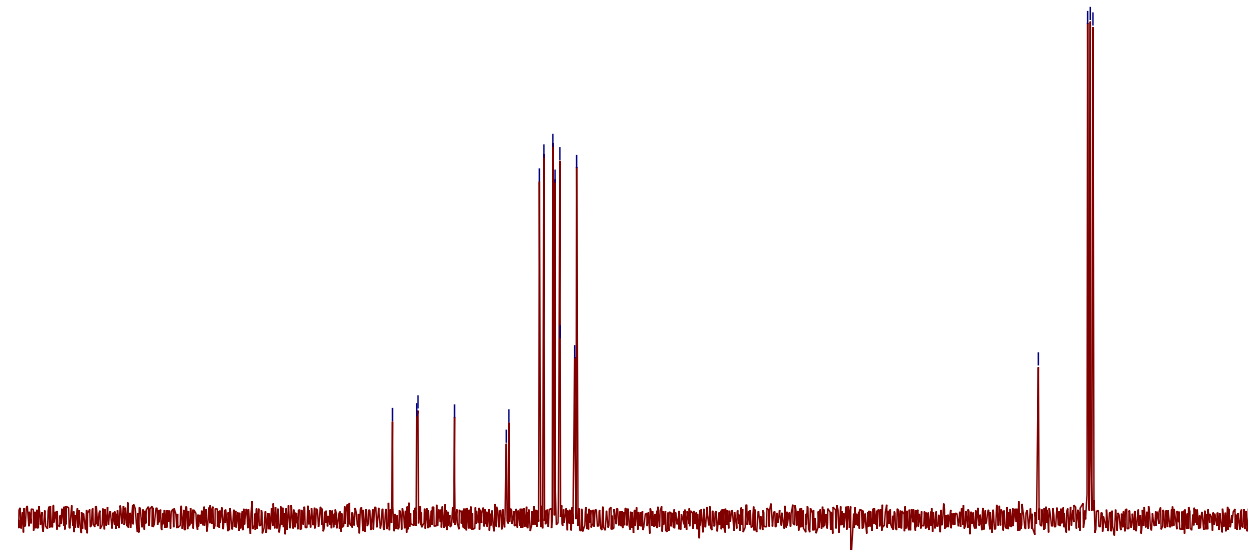

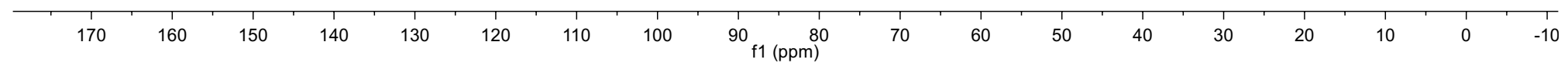


응

웅ㅁำ

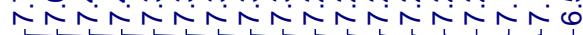

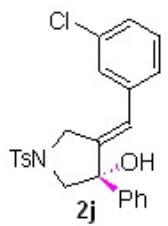

${ }^{1} \mathrm{H} \mathrm{NMR}\left(500 \mathrm{MHz}, \mathrm{CDCl}_{3}\right)$

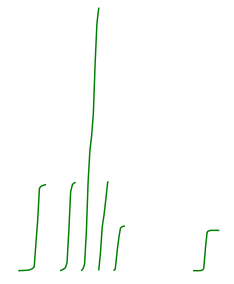

మిల్లిల్లి

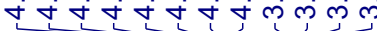

m̧

N

\section{I $\int$}
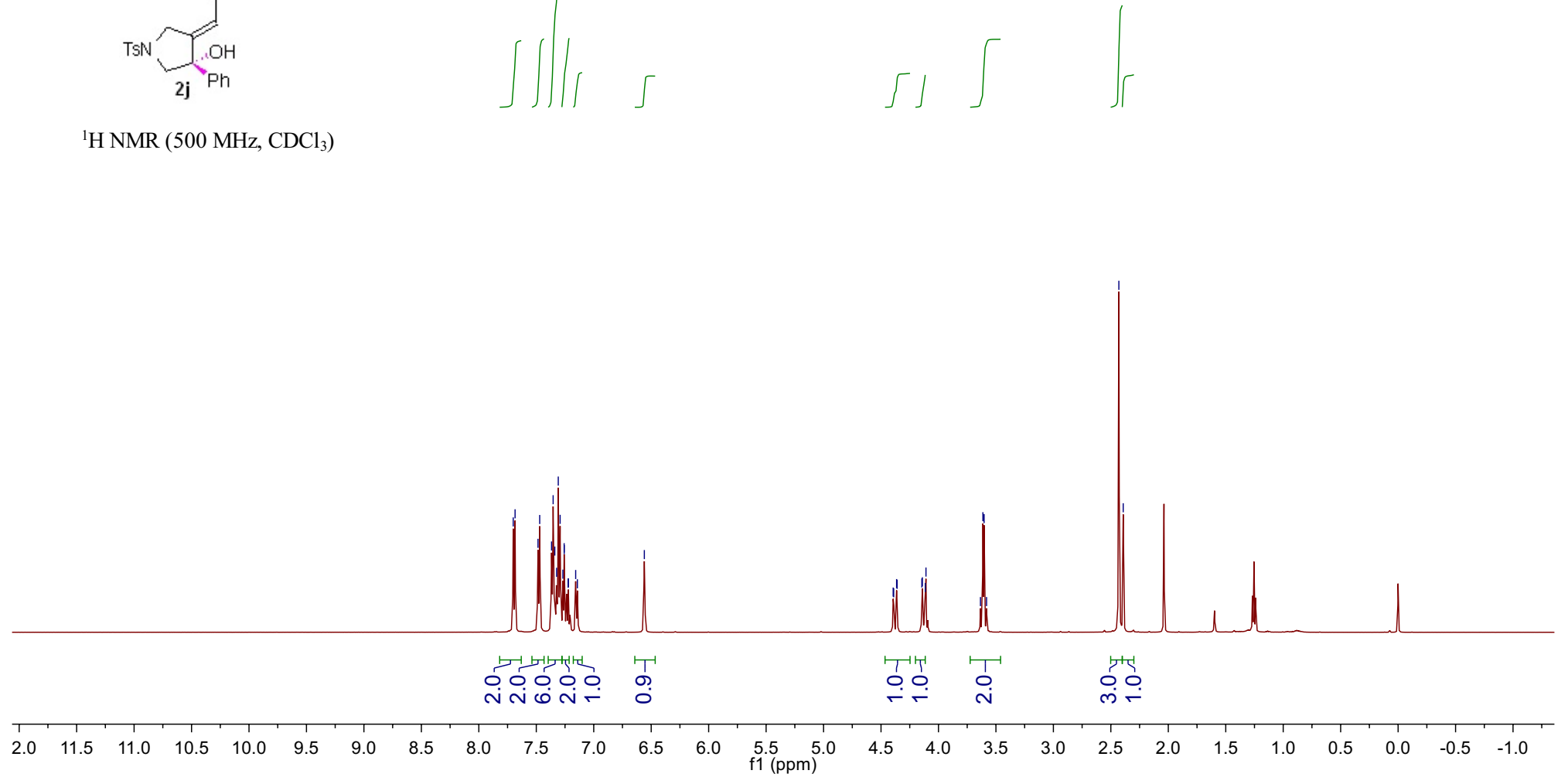


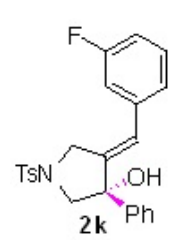

${ }^{1} \mathrm{H}$ NMR $\left(500 \mathrm{MHz}, \mathrm{CDCl}_{3}\right)$
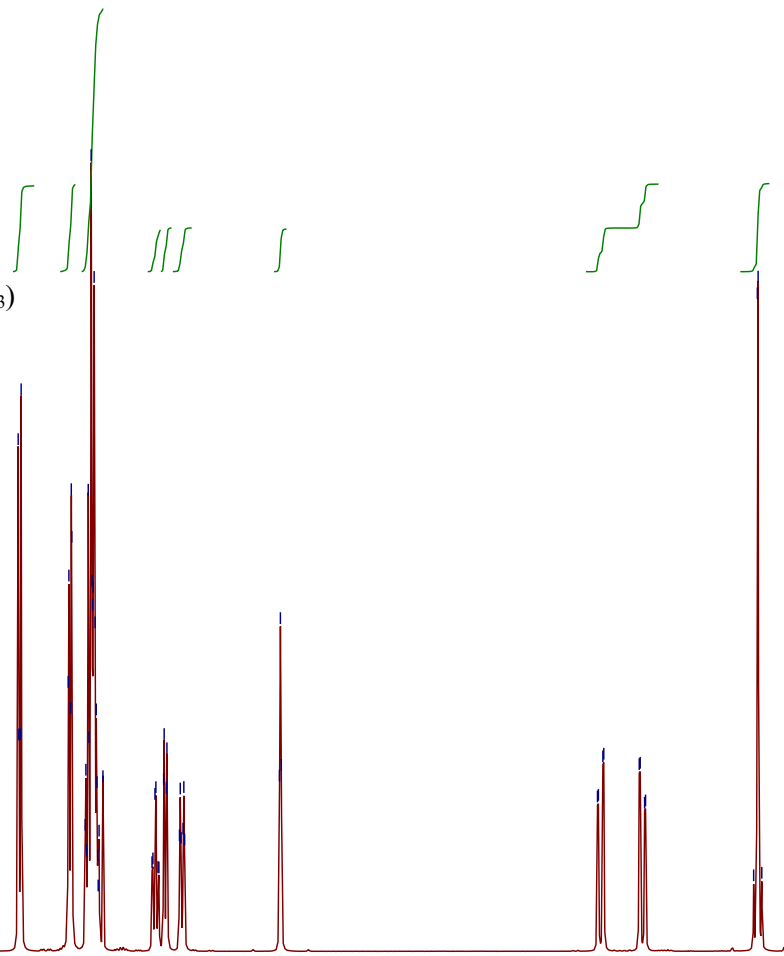

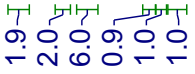

$$
\stackrel{+}{\circ}
$$

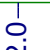

गु




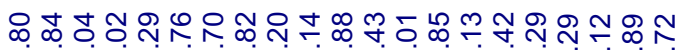
ֻั

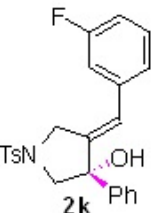

${ }^{13} \mathrm{C}$ NMR (126 MHz, $\left.\mathrm{CDCl}_{3}\right)$

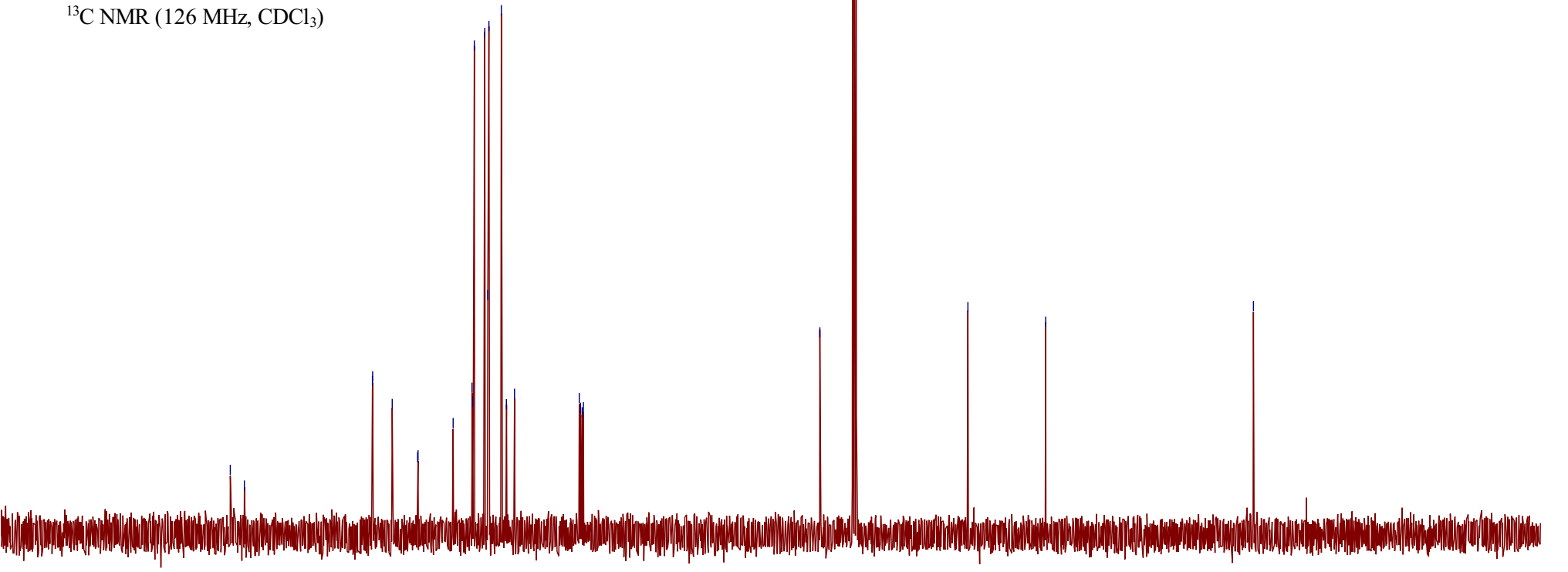

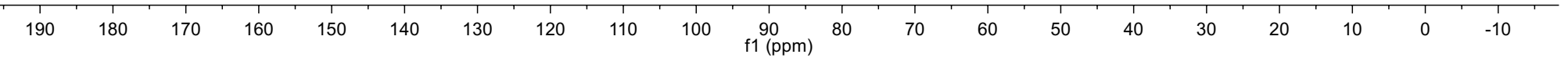


은

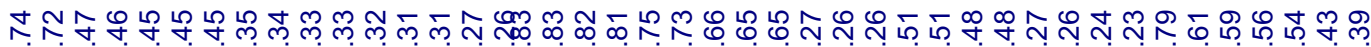

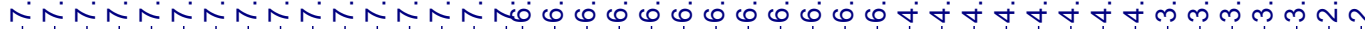

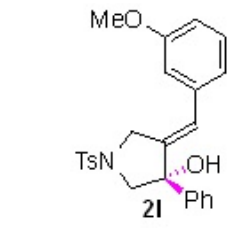

${ }^{1} \mathrm{H}$ NMR (500 MHz, $\mathrm{CDCl}_{3}$ )
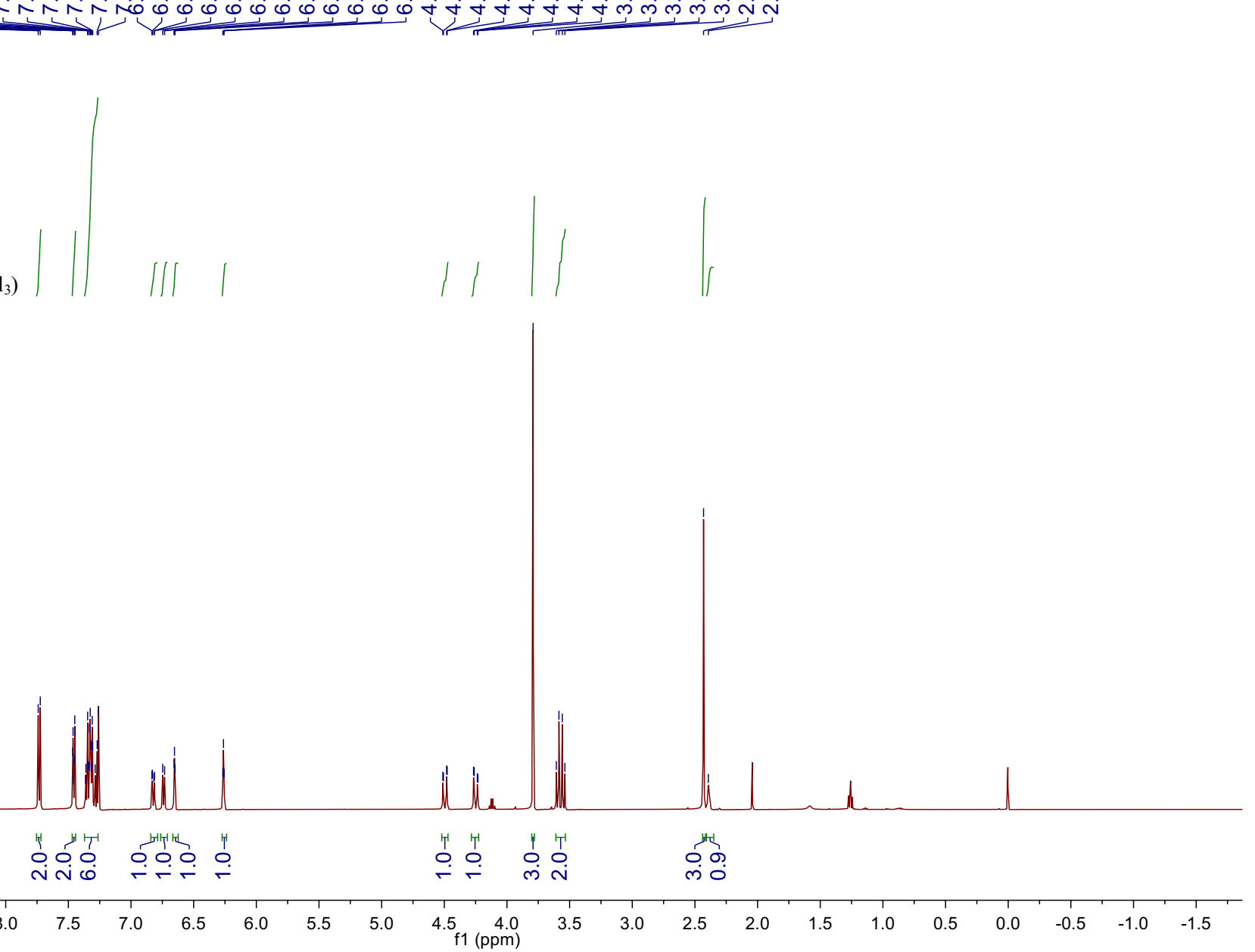


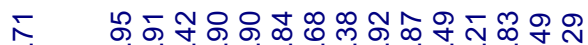

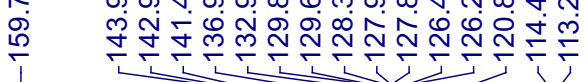

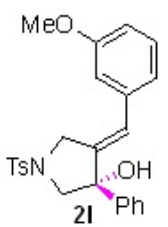

${ }^{13} \mathrm{C}$ NMR (126 MHz, $\left.\mathrm{CDCl}_{3}\right)$ $\frac{m}{\cup} \frac{m}{\cup}$

8

ก

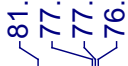

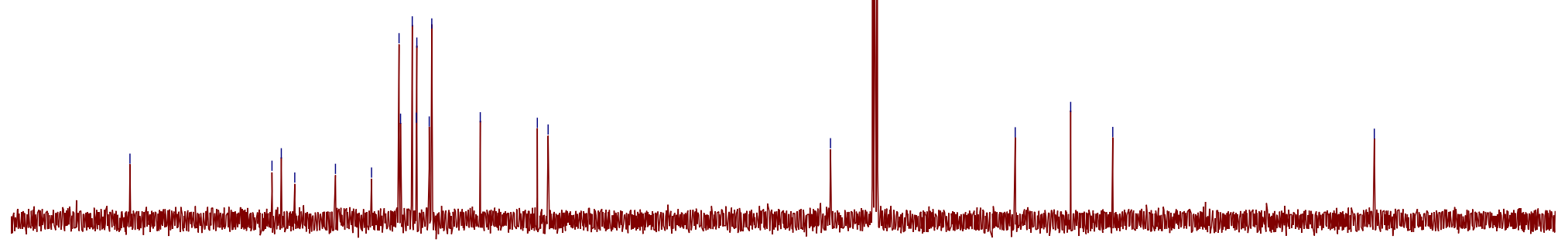

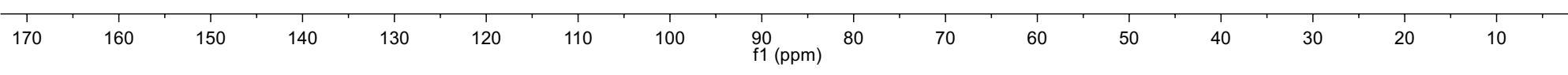




\section{ํㅡㄴ}

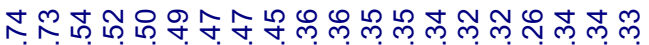

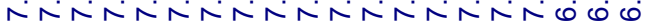

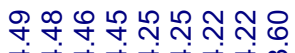

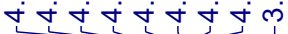

ষே ?

N N

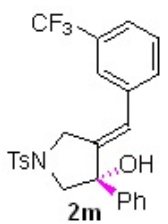

${ }^{1} \mathrm{H} \mathrm{NMR}\left(500 \mathrm{MHz}, \mathrm{CDCl}_{3}\right)$
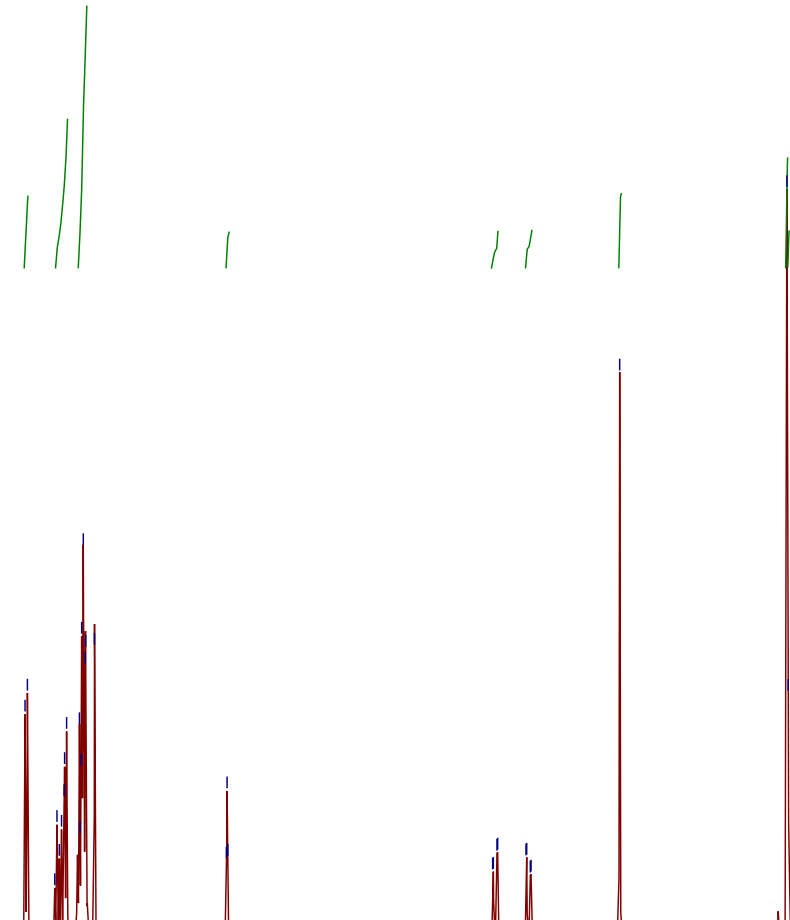

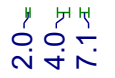

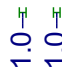

$\stackrel{1}{i}$

이으 


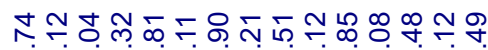

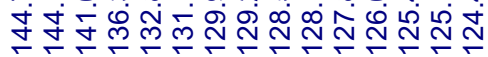

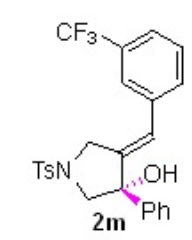

${ }^{13} \mathrm{C}$ NMR (126 MHz, $\mathrm{CDCl}_{3}$ )

\section{$\frac{m}{2} \frac{m}{c} \frac{m}{0}$ \\ ชิ ชิ \\ $\varpi \wedge$ ऽ \\ क쇼 \\ $<1$}

$\frac{\mathfrak{0}}{\mathfrak{1}}$

ֻั

$\stackrel{\infty}{\circ}$

זั
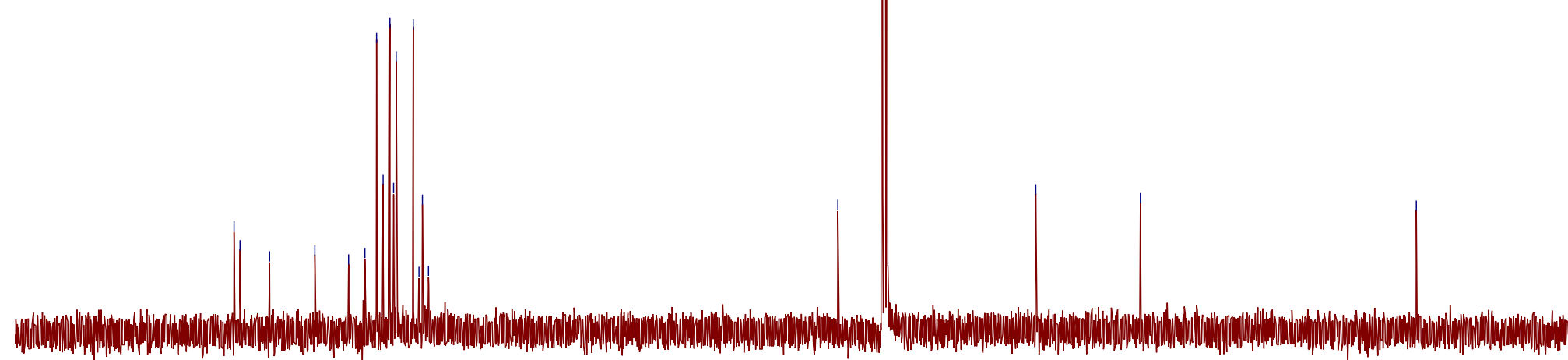

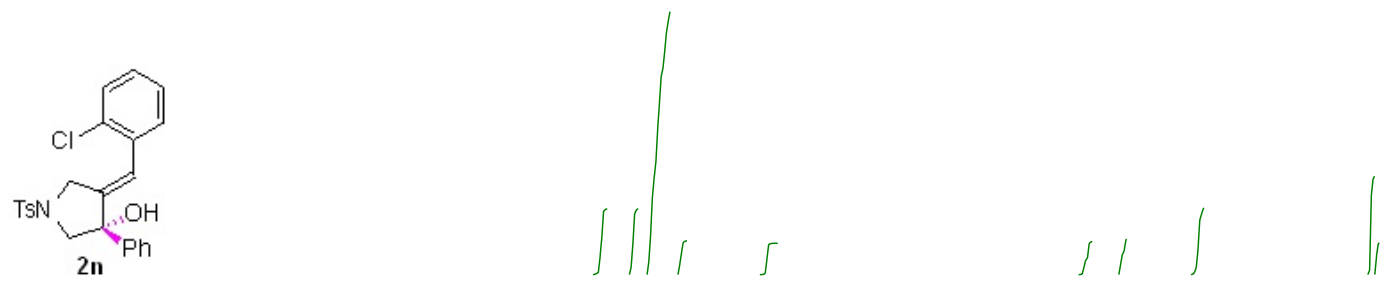

${ }^{1} \mathrm{H}$ NMR (500 MHz, $\left.\mathrm{CDCl}_{3}\right)$

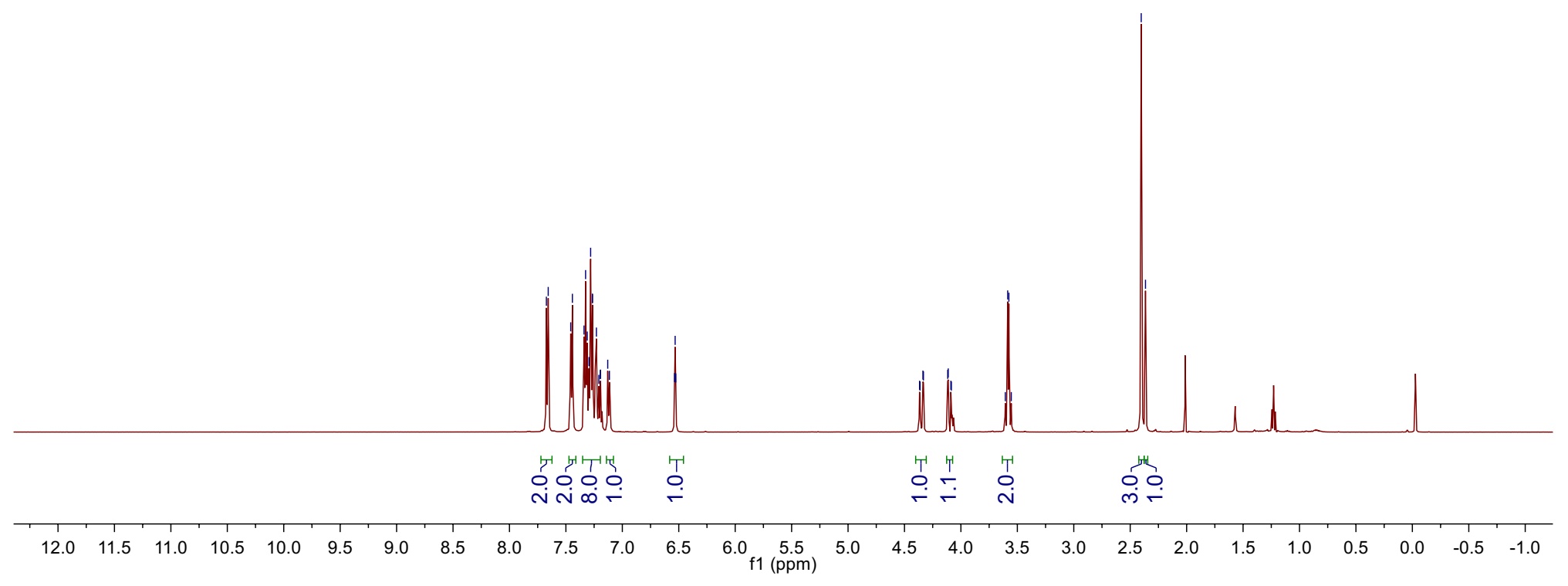




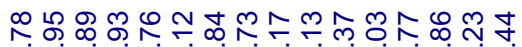
守守守苮岕

Ti

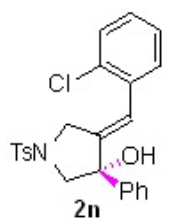

${ }^{13} \mathrm{C}$ NMR (126 MHz, $\mathrm{CDCl}_{3}$ )

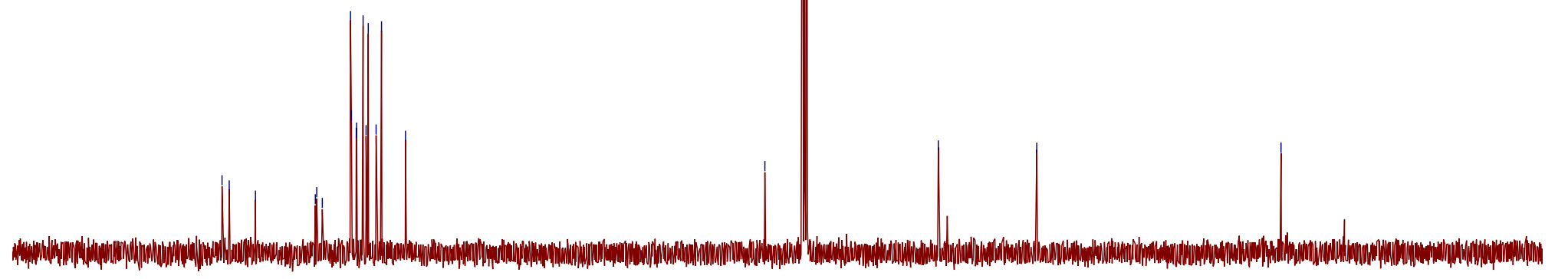

$\begin{array}{llllllll}150 & 140 & 130 & 120 & 110 & 100 & 90 & \left.\begin{array}{l}80 \\ \mathrm{f} 1(\mathrm{ppm})\end{array}\right)\end{array}$

$70 \quad 60$

50



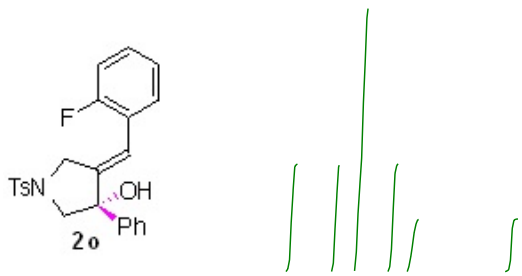

${ }^{1} \mathrm{H} \mathrm{NMR}\left(500 \mathrm{MHz}, \mathrm{CDCl}_{3}\right)$
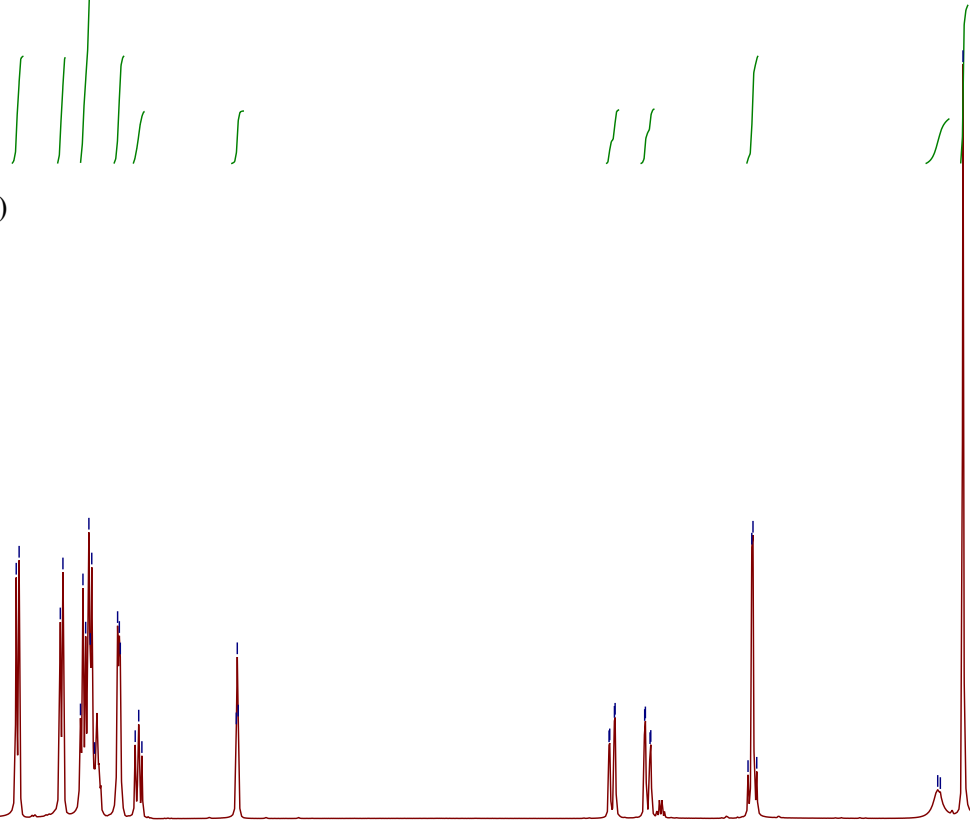

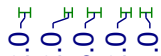

$\stackrel{+T}{\circ}$

个1

웅

$\stackrel{T}{\mathfrak{O}^{\prime}}$

क़่

$\begin{array}{lll}4.0 & 3.5 & 3.0\end{array}$

2.5

2.0


¿ேீ

ธำ

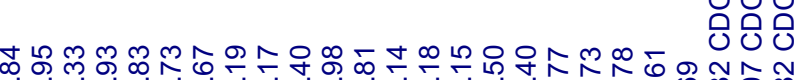

$1 \frac{1}{1}$

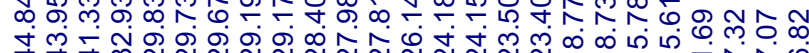

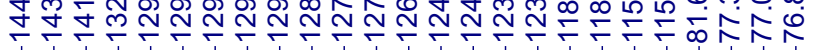

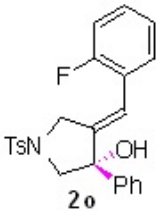

${ }^{13} \mathrm{C}$ NMR $\left(126 \mathrm{MHz}, \mathrm{CDCl}_{3}\right)$

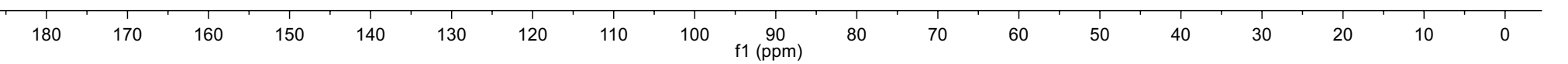



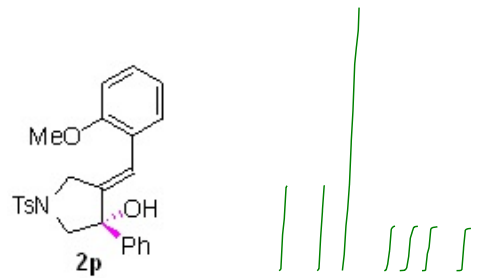

${ }^{1} \mathrm{H}$ NMR (500 MHz, $\mathrm{CDCl}_{3}$ )
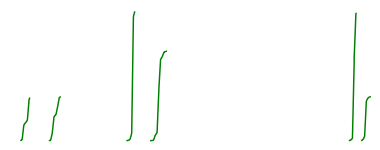

( 

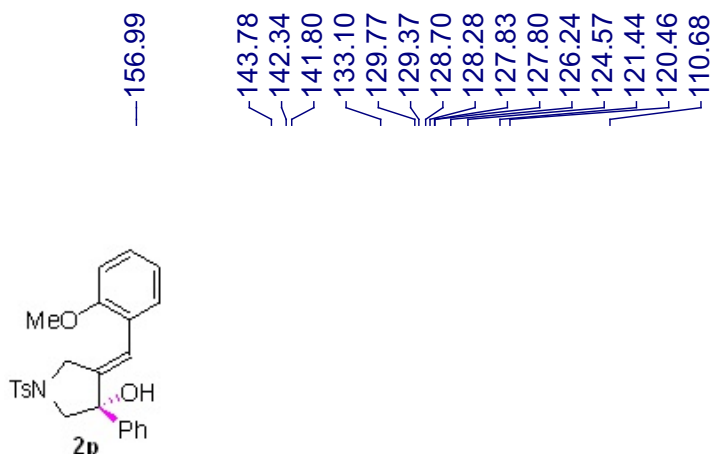

${ }^{13} \mathrm{C}$ NMR (126 MHz, $\mathrm{CDCl}_{3}$ )

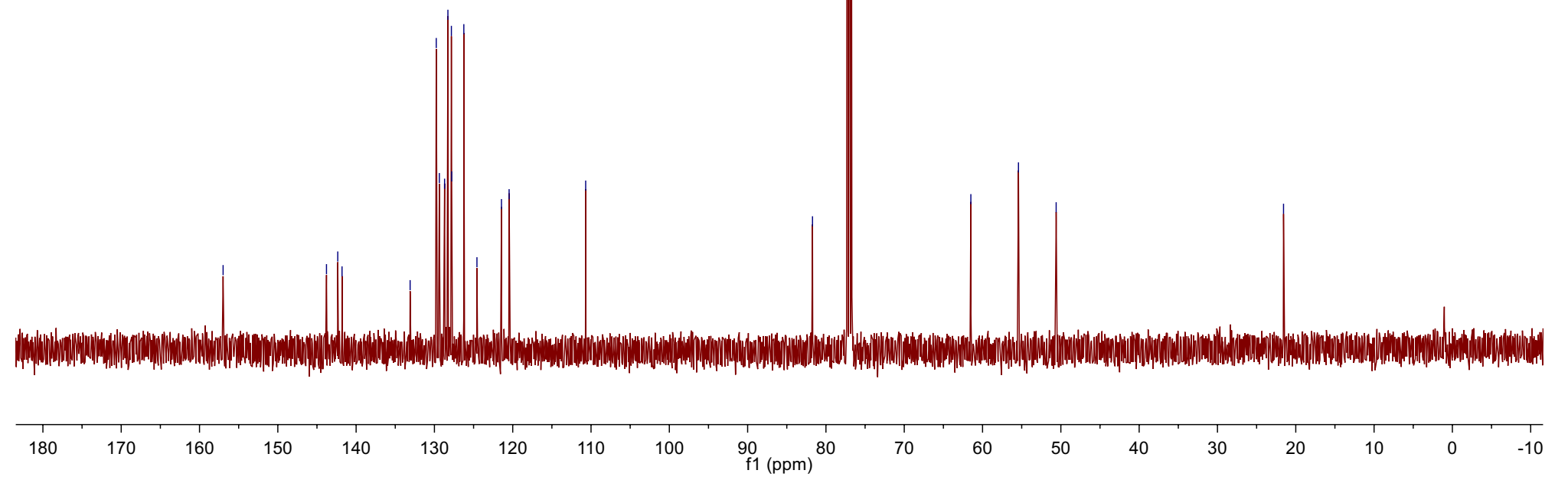




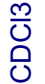

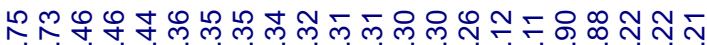

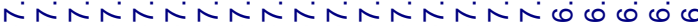

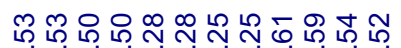

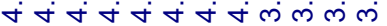

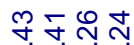

Nin
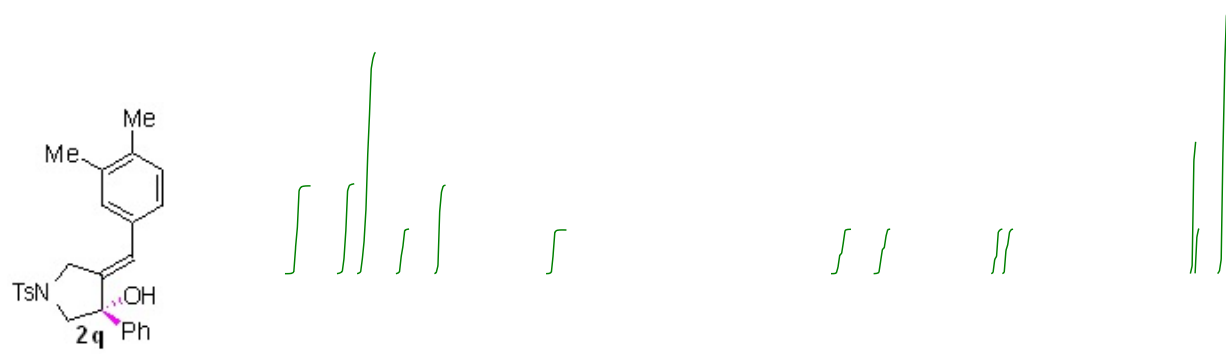

${ }^{1} \mathrm{H}$ NMR $\left(500 \mathrm{MHz}, \mathrm{CDCl}_{3}\right)$

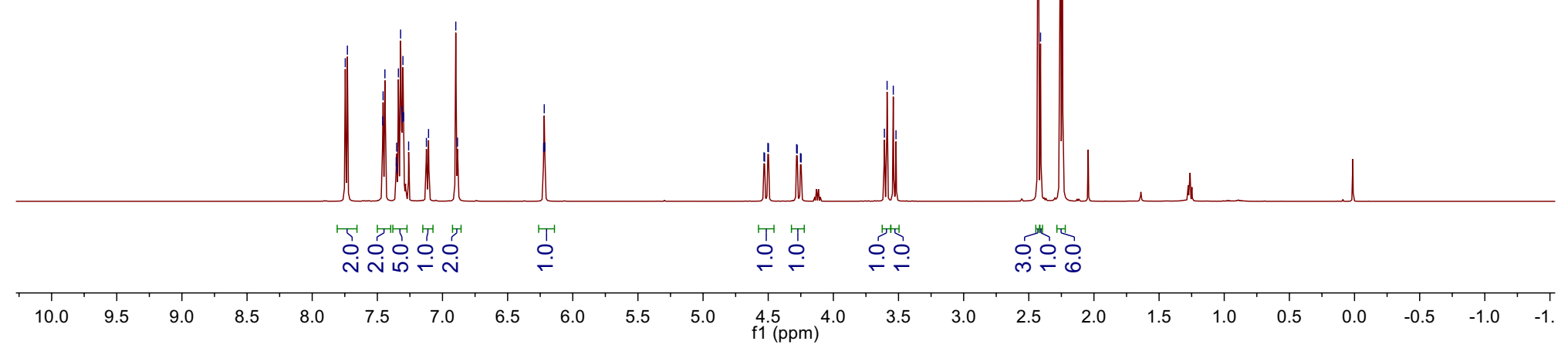




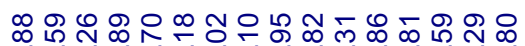

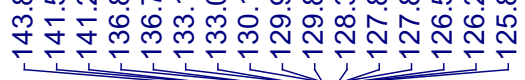

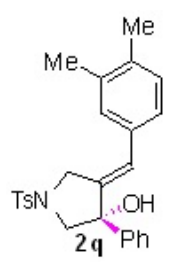

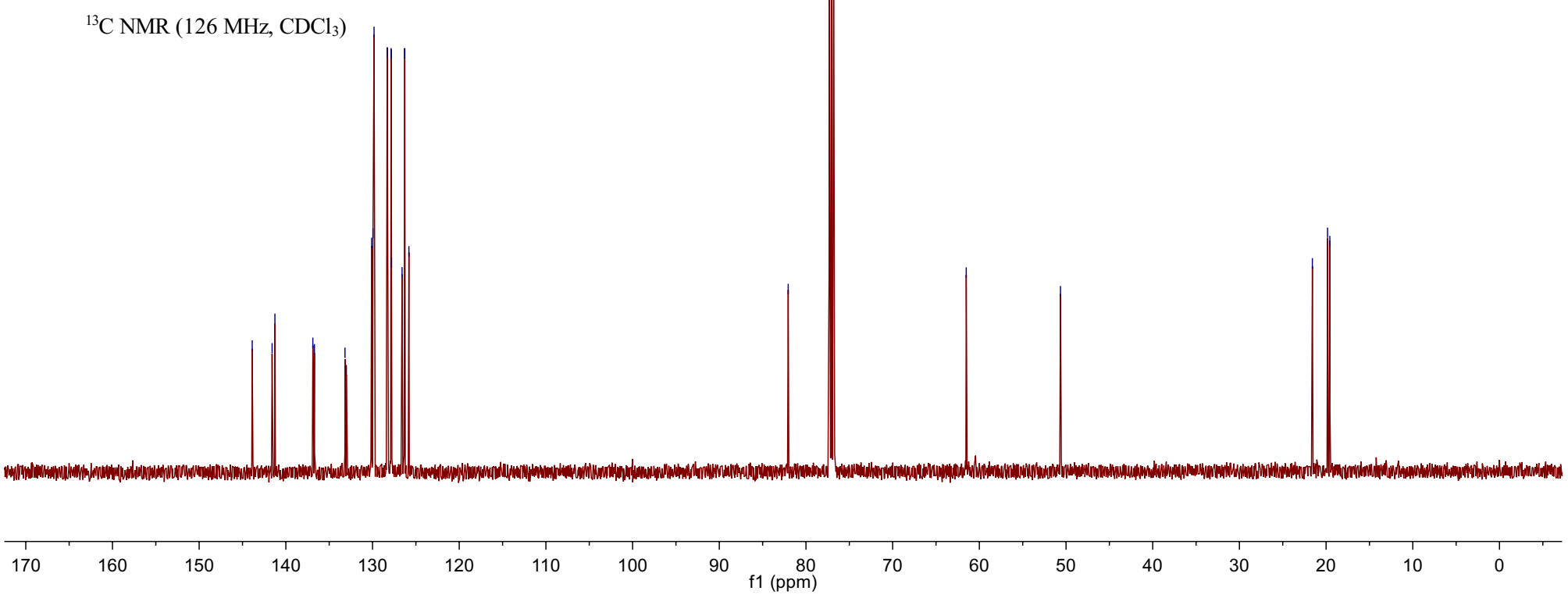




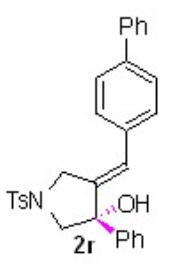

${ }^{1} \mathrm{H} \mathrm{NMR}\left(500 \mathrm{MHz}, \mathrm{CDCl}_{3}\right)$
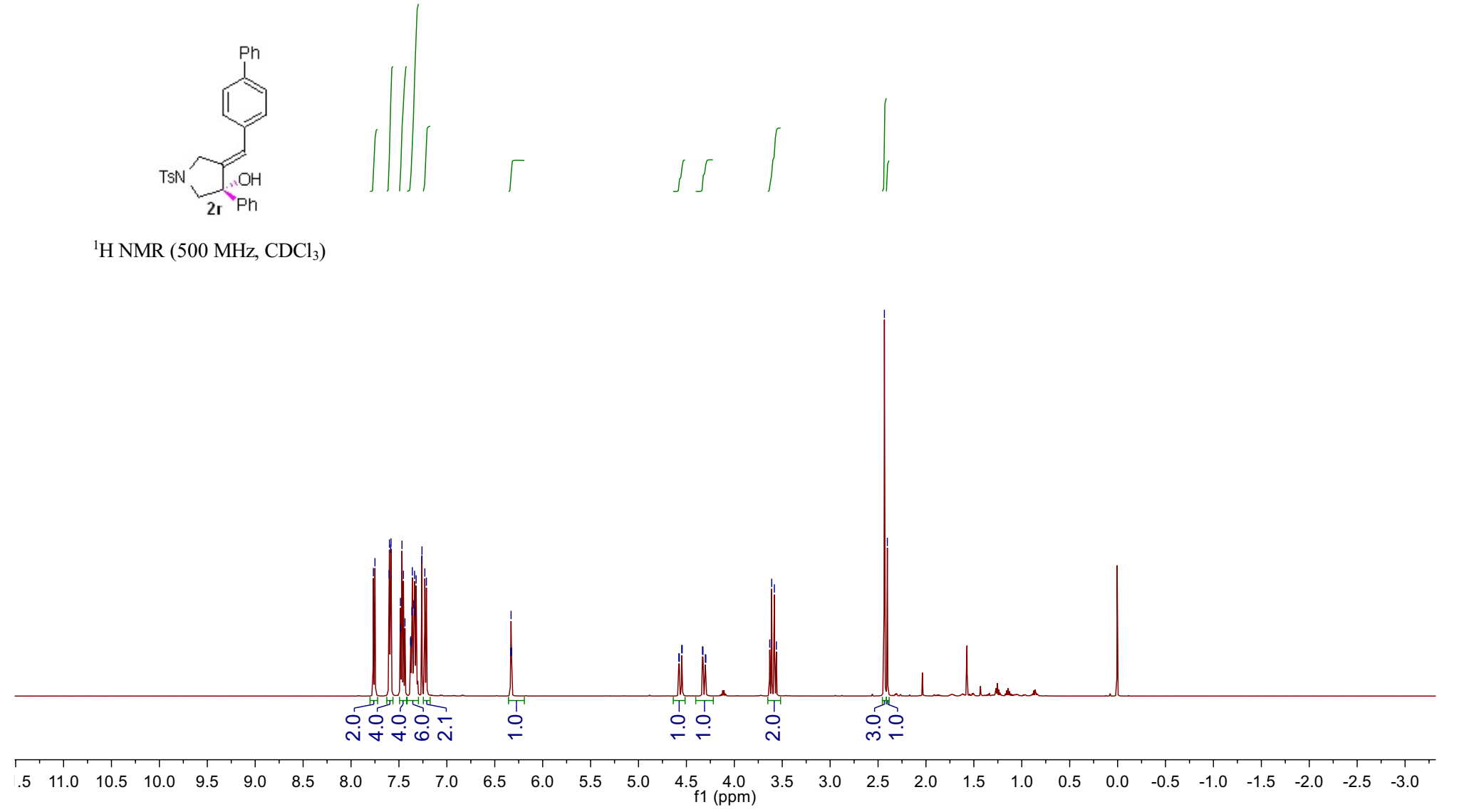


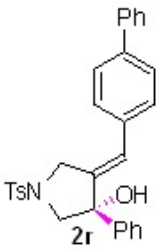

${ }^{13} \mathrm{C} \mathrm{NMR}\left(126 \mathrm{MHz}, \mathrm{CDCl}_{3}\right)$

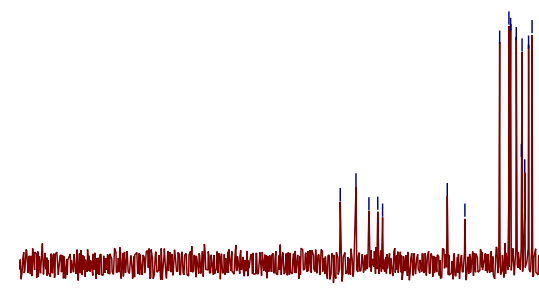


은

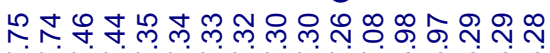

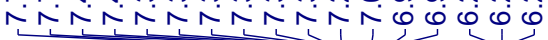

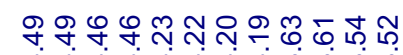

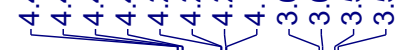

$\stackrel{\infty}{+\infty} \underset{+}{+}$

i

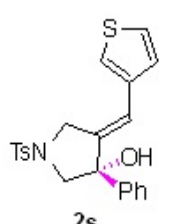

2s

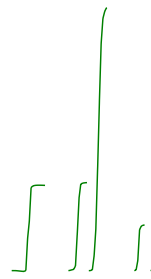

$\iint \quad \int$

H NMR (500 MHz, $\mathrm{CDCl}_{3}$ )
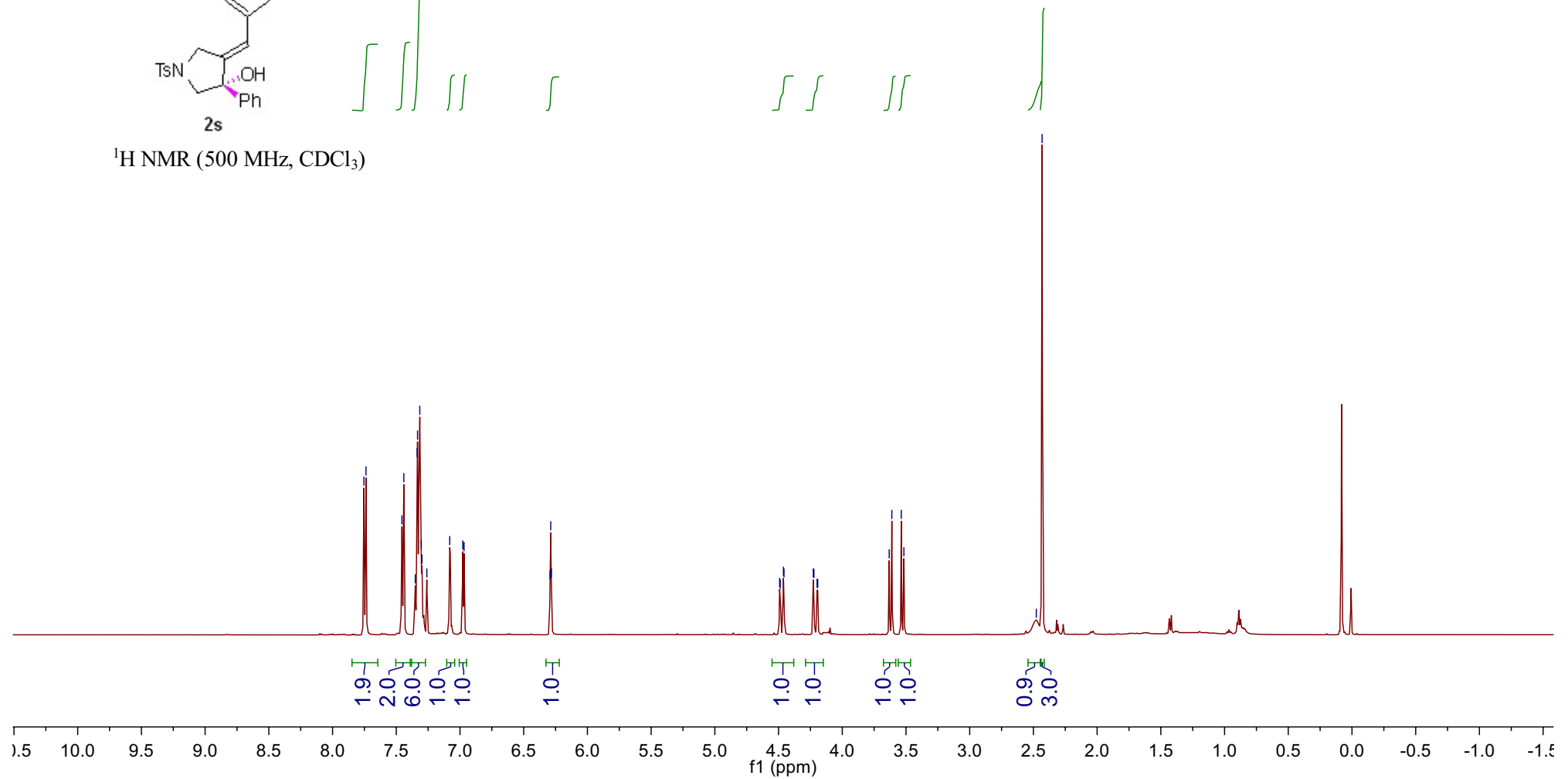


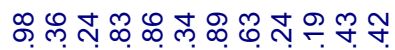

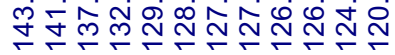

${ }^{2}$

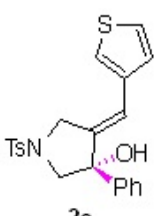

2s

${ }^{13} \mathrm{C} \mathrm{NMR}\left(126 \mathrm{MHz}, \mathrm{CDCl}_{3}\right)$

ดัด

ㅇำ

六

ris

ㅇ.

$\bar{\emptyset}$

8

in

त

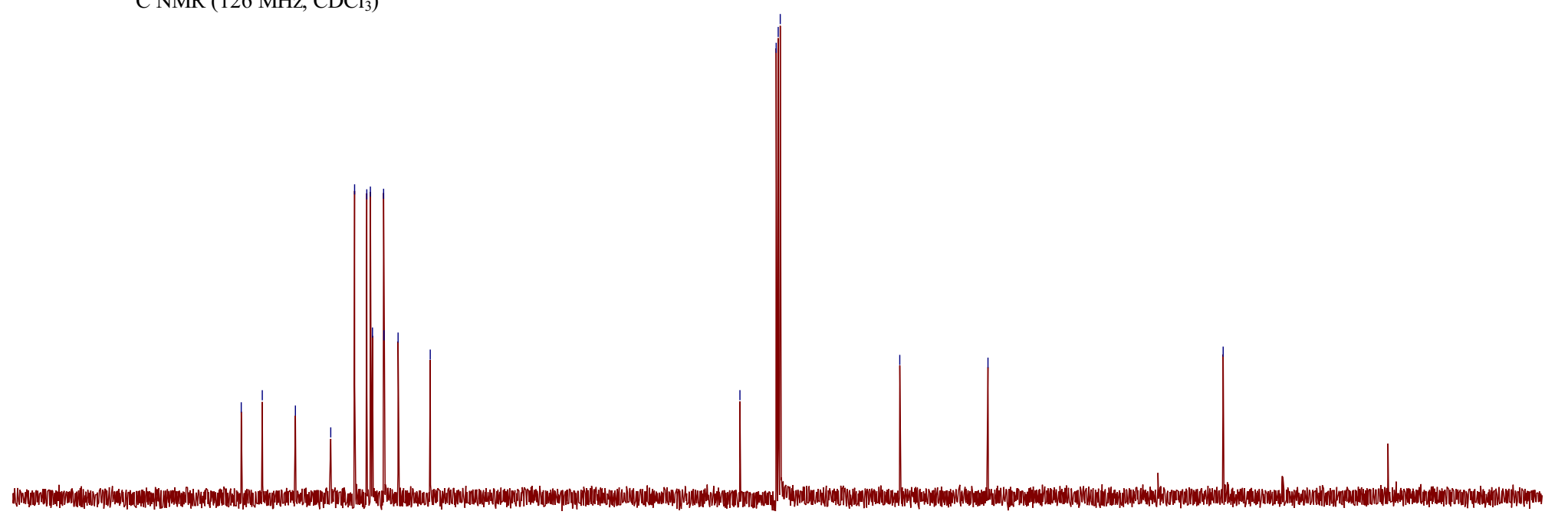



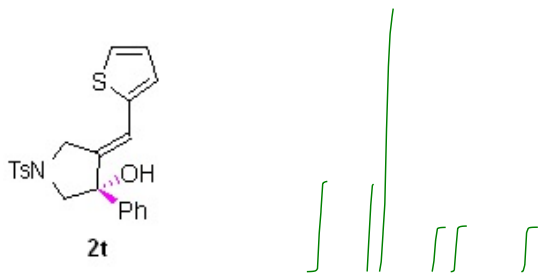

${ }^{1} \mathrm{H} \mathrm{NMR}\left(500 \mathrm{MHz}, \mathrm{CDCl}_{3}\right)$

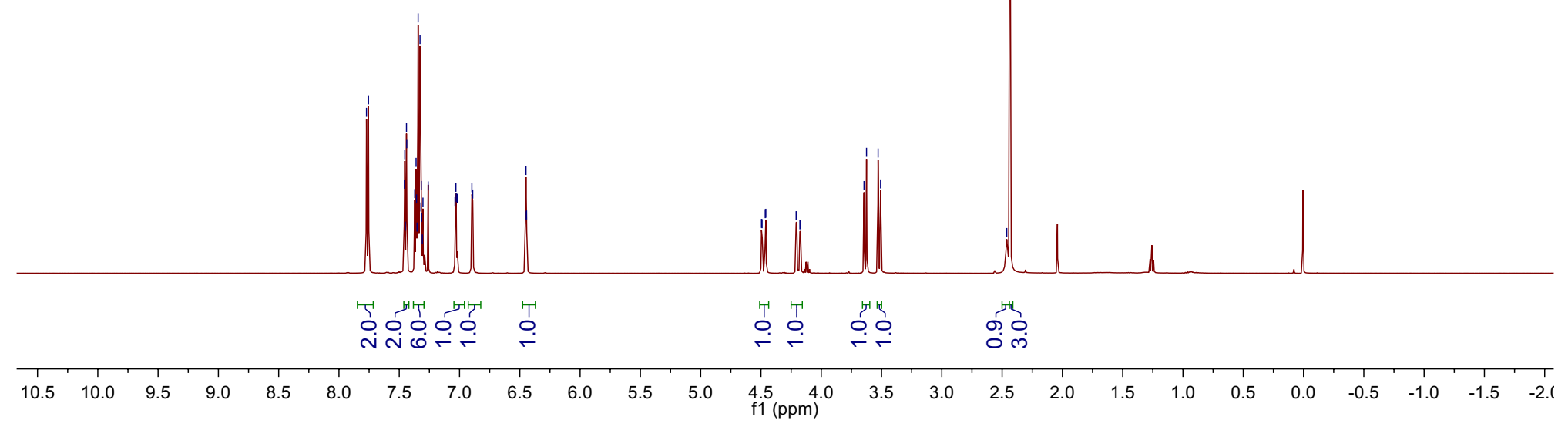




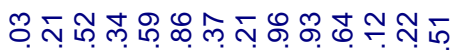
守守守穴

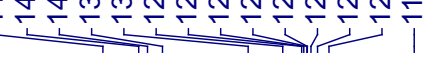

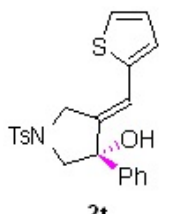

${ }^{13} \mathrm{C}$ NMR (126 MHz, $\left.\mathrm{CDCl}_{3}\right)$

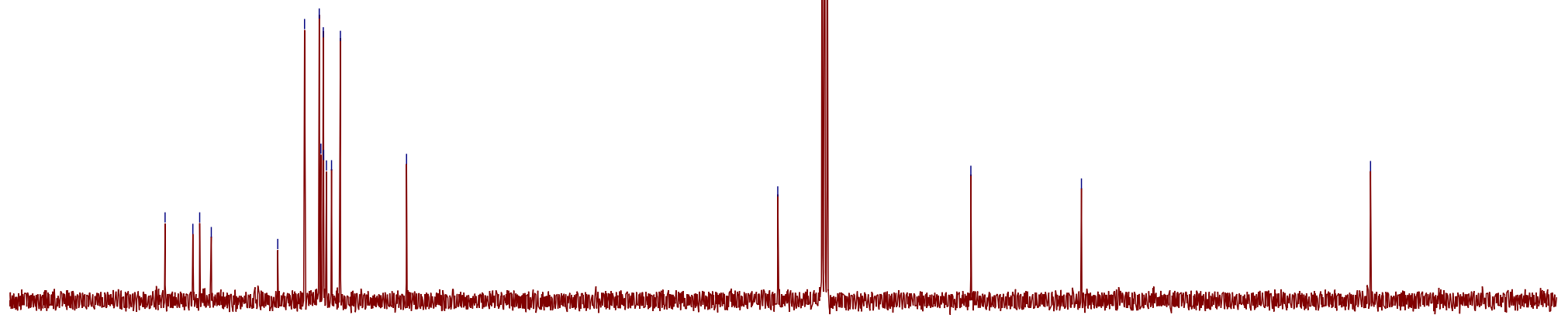

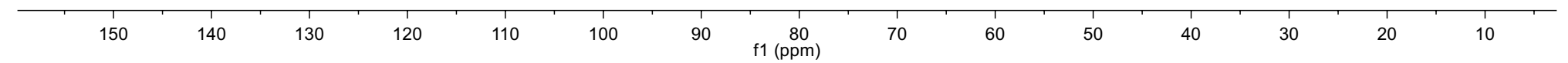


บั

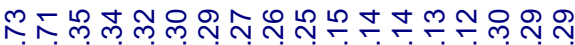

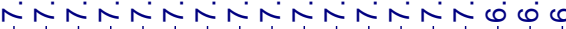

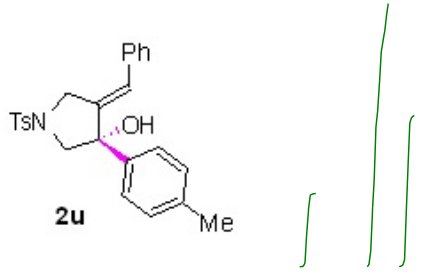

${ }^{1} \mathrm{H}$ NMR (500 MHz, $\mathrm{CDCl}_{3}$ )

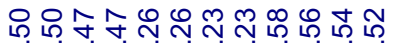

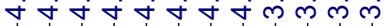

ำ ํำ

กิ

11

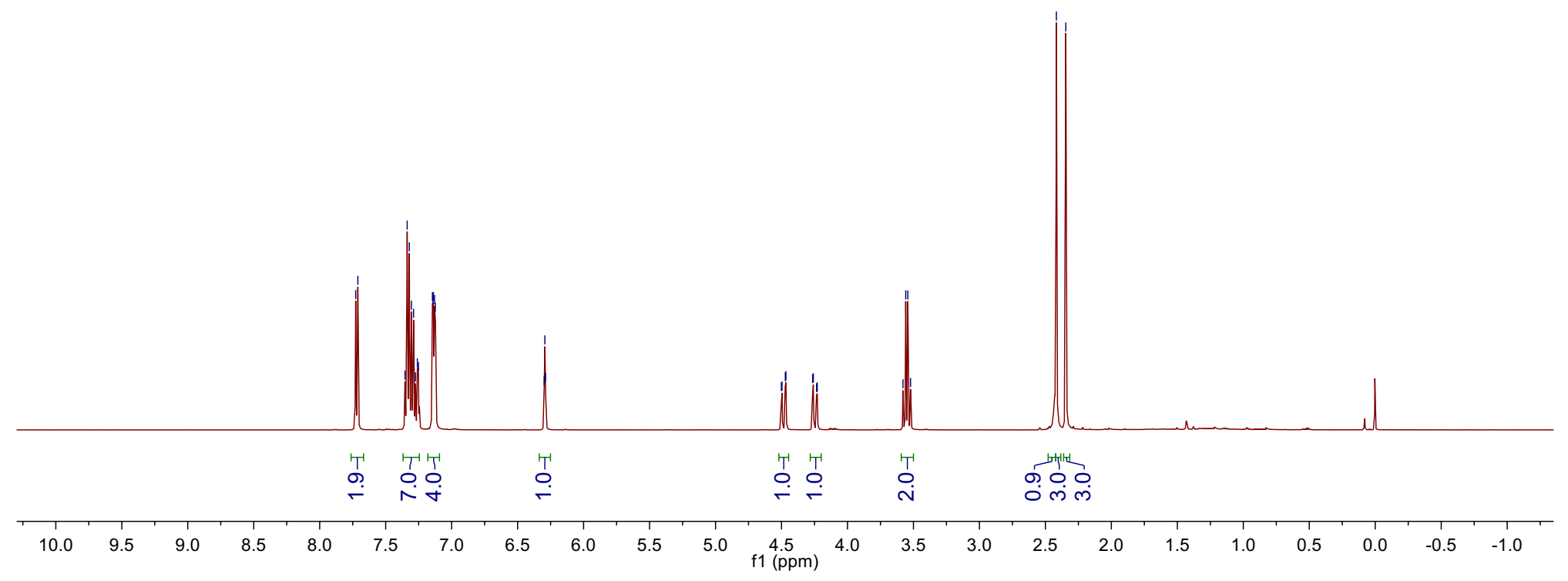


œ

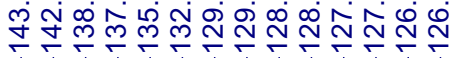

1

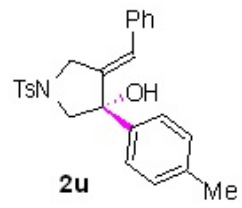

${ }^{13} \mathrm{C}$ NMR (126 MHz, $\left.\mathrm{CDCl}_{3}\right)$

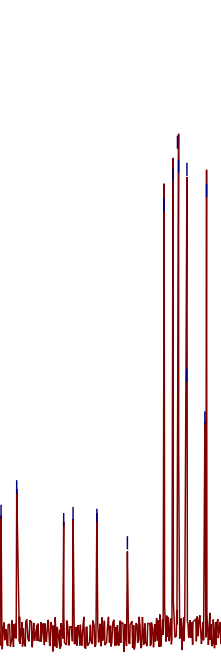


రิ

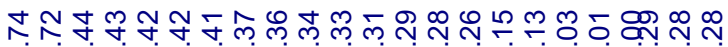

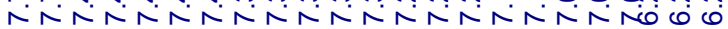

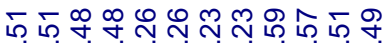

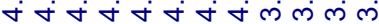

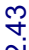

i

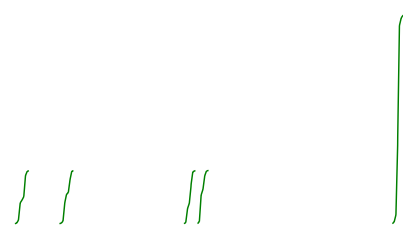

${ }^{1} \mathrm{H}$ NMR (500 MHz, $\mathrm{CDCl}_{3}$ )

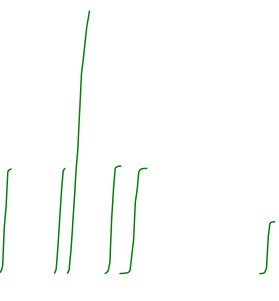

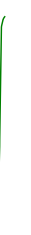

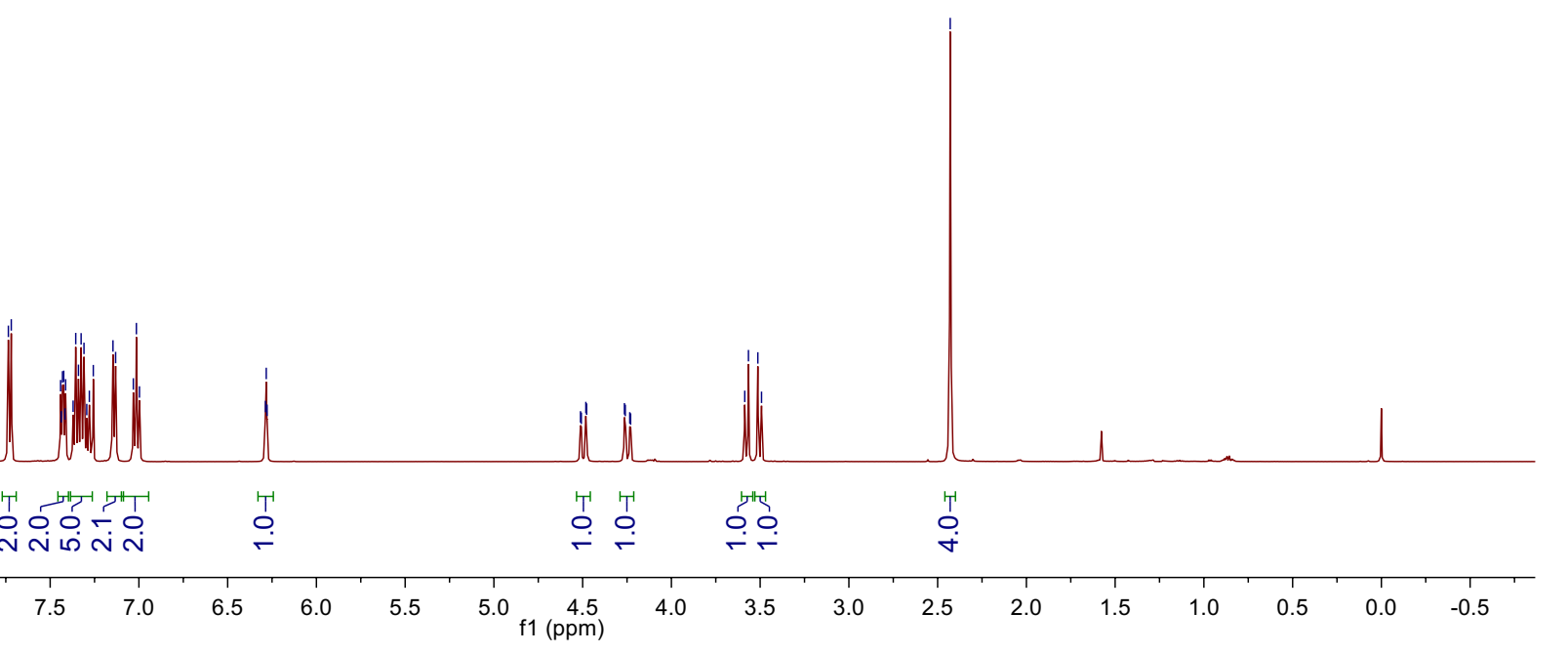




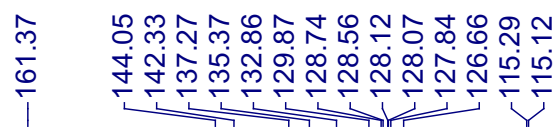

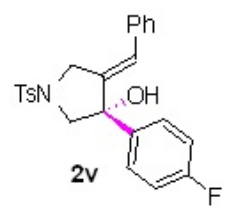

${ }^{13} \mathrm{C}$ NMR (126 MHz, $\mathrm{CDCl}_{3}$ )

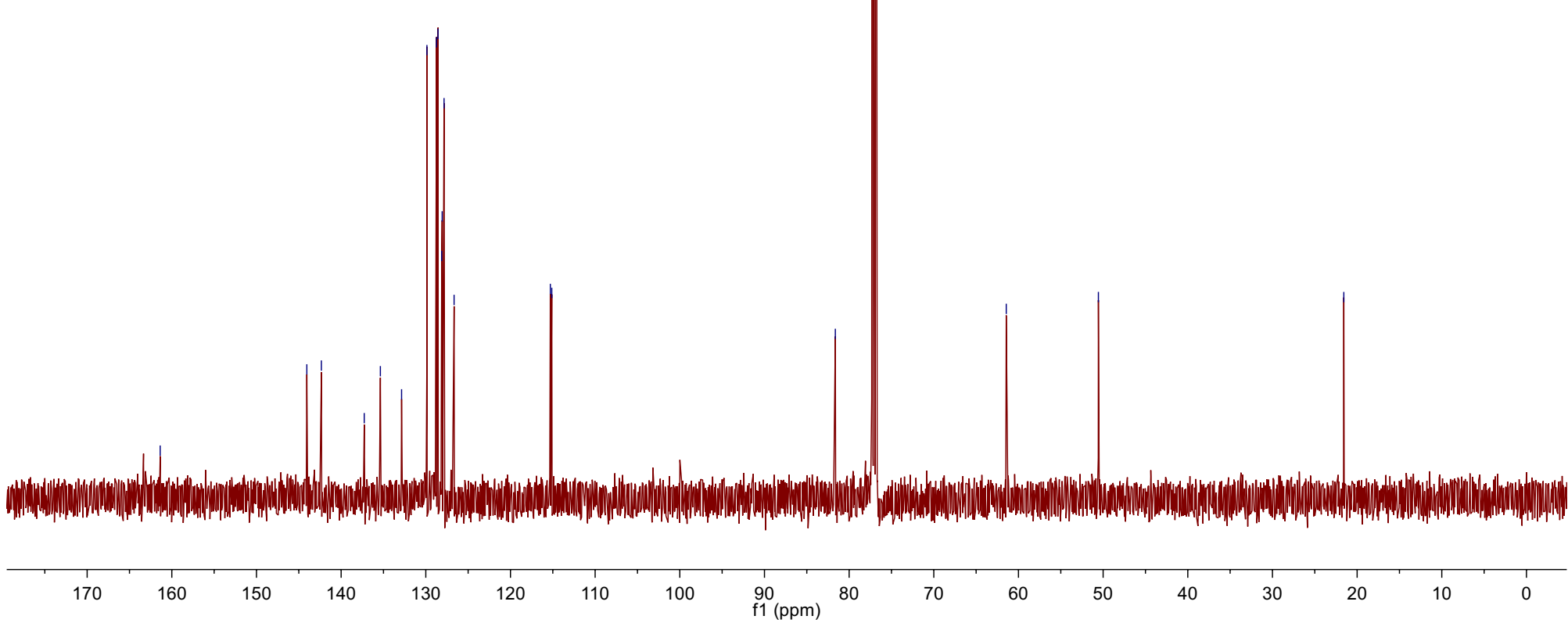



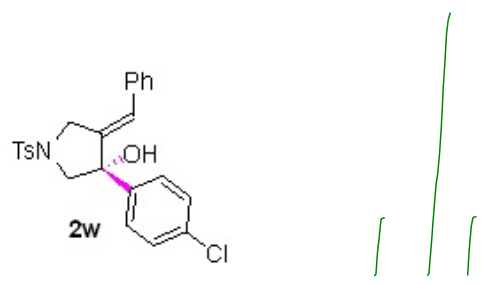

${ }^{1} \mathrm{H} \mathrm{NMR}\left(500 \mathrm{MHz}, \mathrm{CDCl}_{3}\right)$

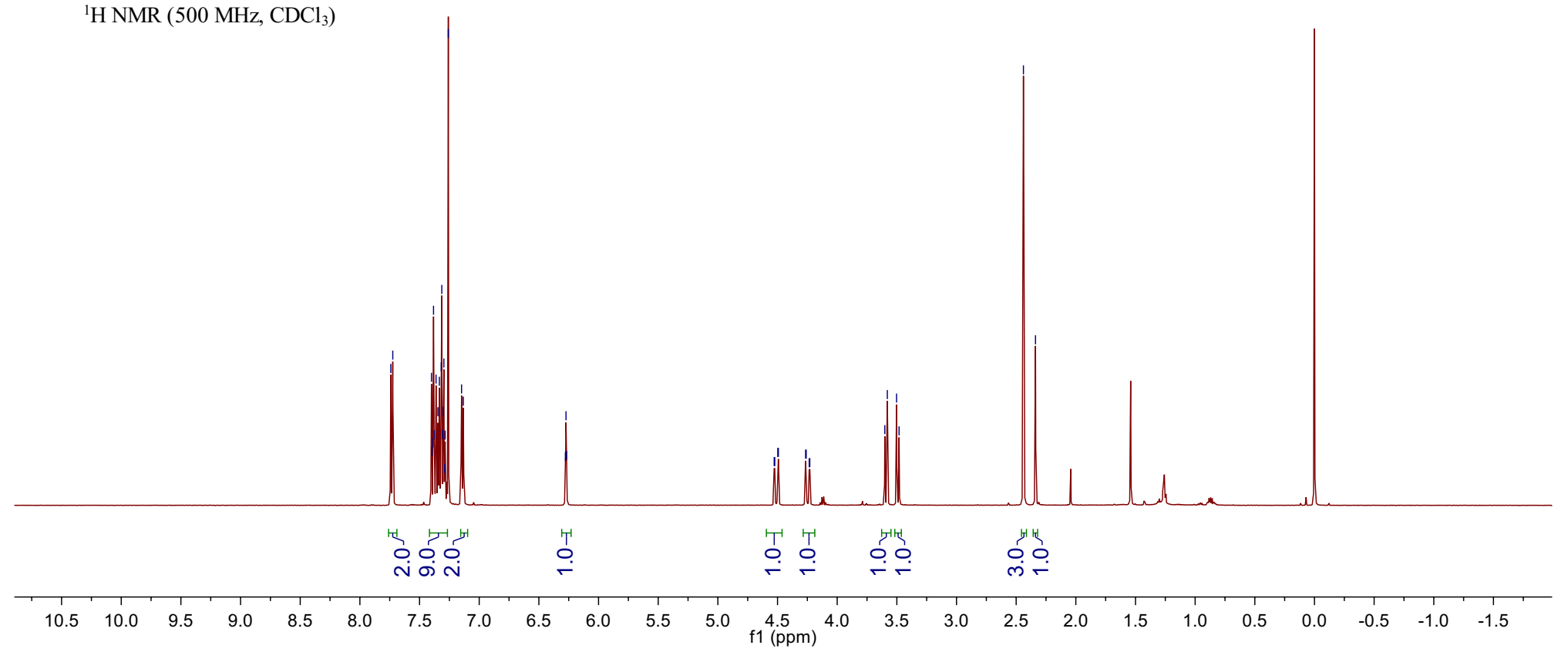




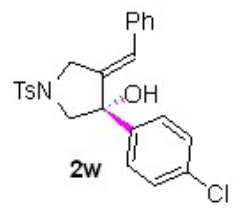

${ }^{13} \mathrm{C}$ NMR (126 MHz, $\mathrm{CDCl}_{3}$ )

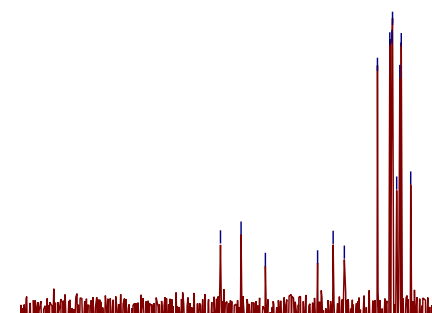


$\frac{m}{0}$

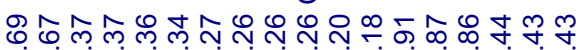

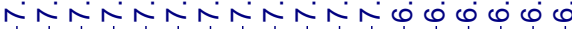

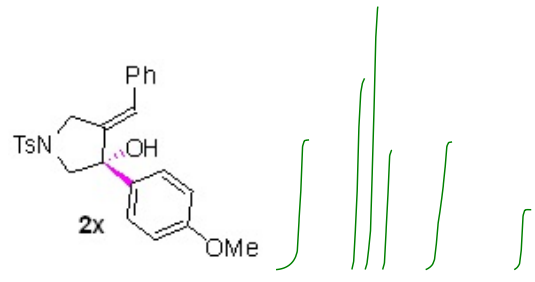

${ }^{1} \mathrm{H} \mathrm{NMR}\left(500 \mathrm{MHz}, \mathrm{CDCl}_{3}\right)$

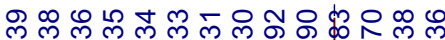

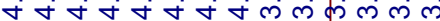

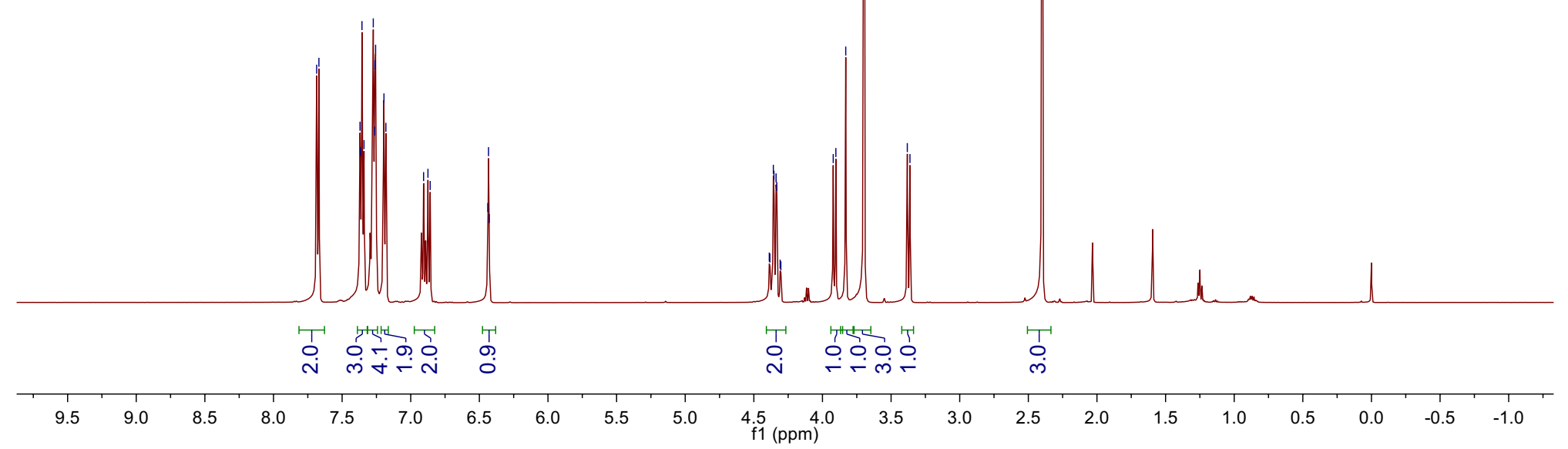




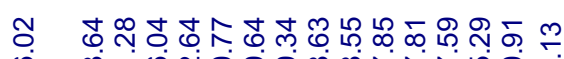

î

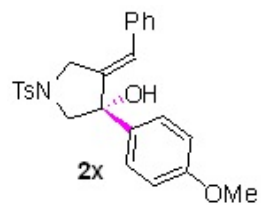

${ }^{13} \mathrm{C}$ NMR (126 MHz, $\mathrm{CDCl}_{3}$ ) $\frac{m}{0} \frac{m}{0} \frac{m}{0}$

8

๓

次令

유융

용ํㅇ

$<1<$

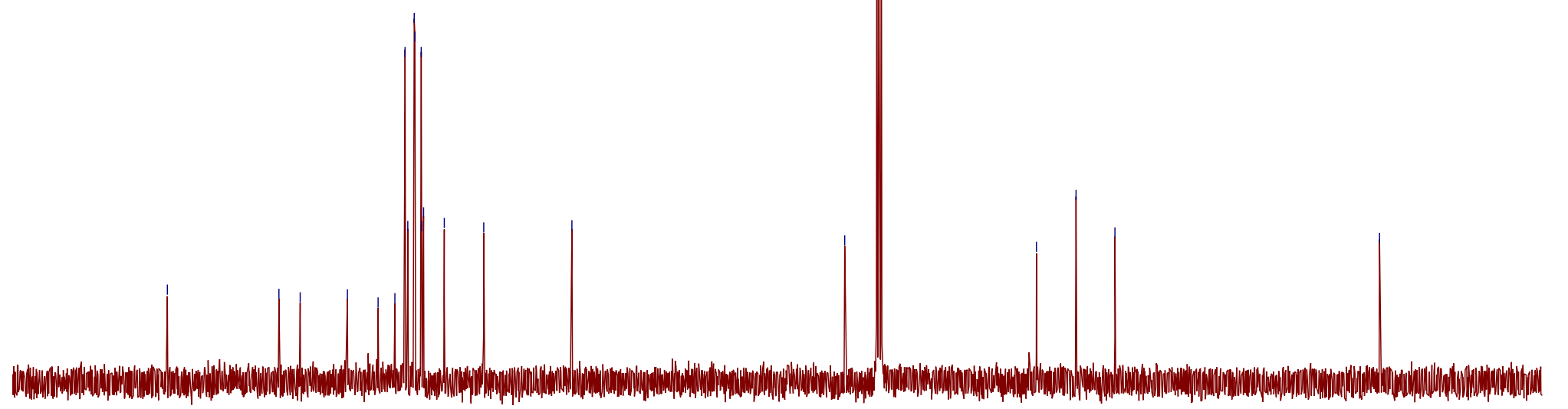

\begin{tabular}{|c|c|c|c|c|c|c|c|c|c|c|c|c|c|c|c|}
\hline 170 & 160 & 150 & 140 & 130 & 120 & 110 & 100 & $\begin{array}{l}90 \\
\mathrm{f} 1(\mathrm{ppm})\end{array}$ & 80 & 70 & 60 & 50 & 40 & 30 & 20 \\
\hline
\end{tabular}


ํํำ

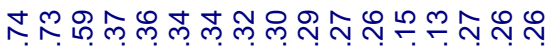

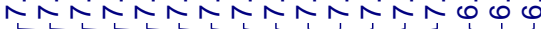

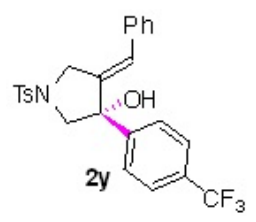

${ }^{1} \mathrm{H}$ NMR (500 MHz, $\mathrm{CDCl}_{3}$ )

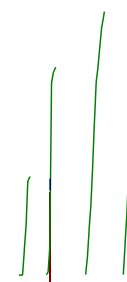

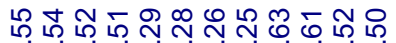

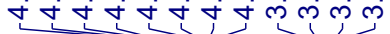

过寺

i
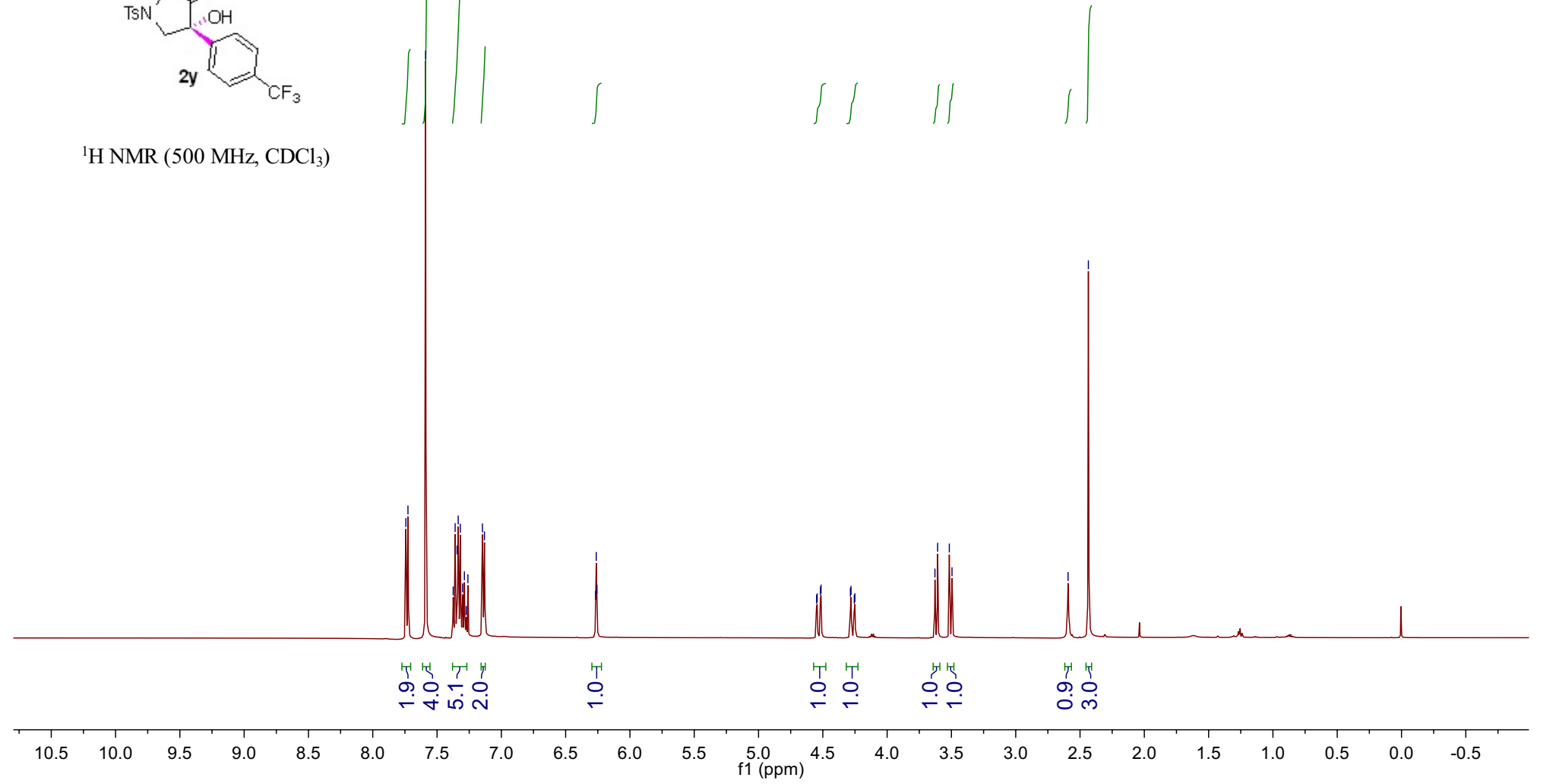
$\frac{m}{\text { U⿱口 }}$

ค

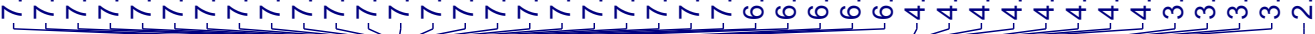

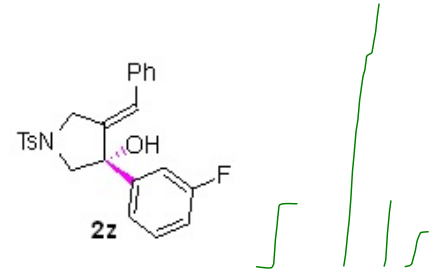

${ }^{1} \mathrm{H} \mathrm{NMR}\left(500 \mathrm{MHz}, \mathrm{CDCl}_{3}\right)$

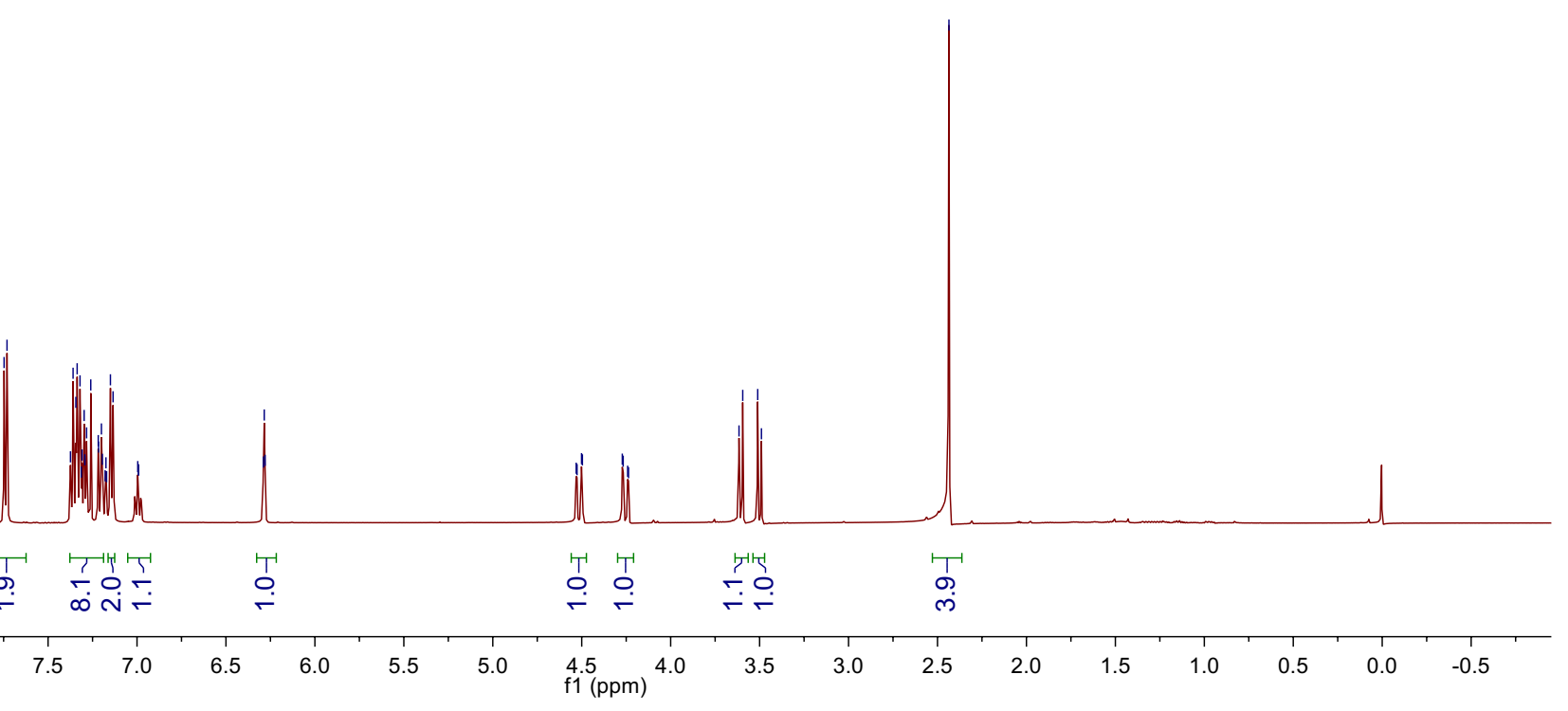




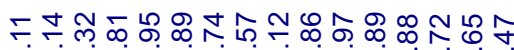

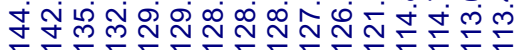
$\sqrt{5+5}$

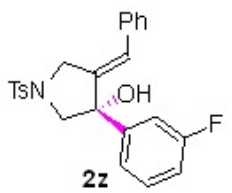

${ }^{13} \mathrm{C}$ NMR (126 MHz, $\left.\mathrm{CDCl}_{3}\right)$

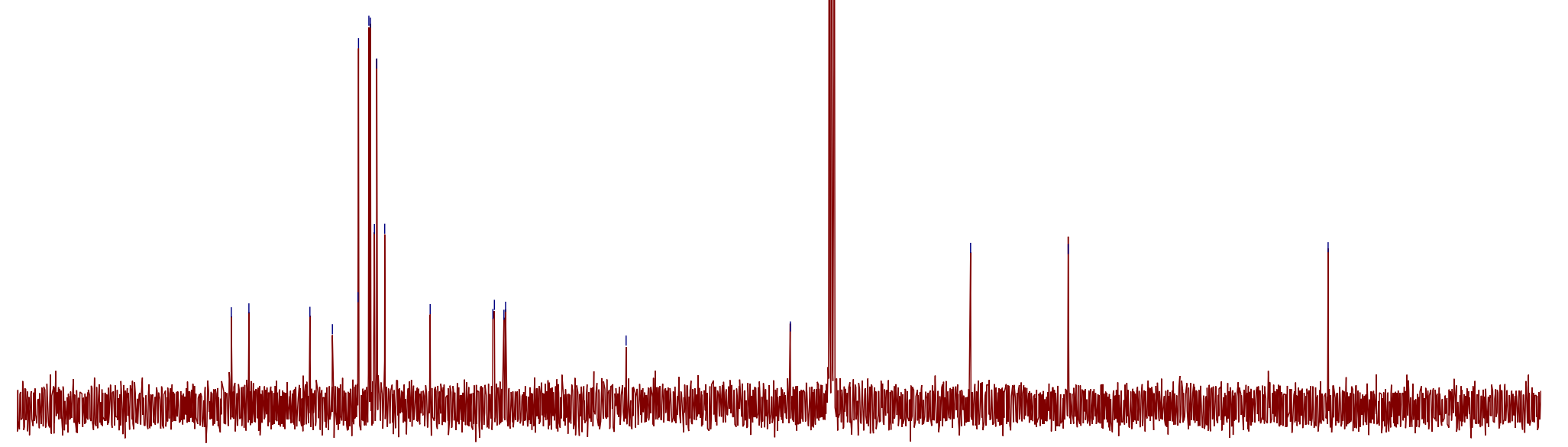




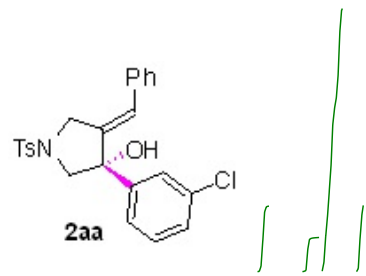

${ }^{1} \mathrm{H} \mathrm{NMR}\left(500 \mathrm{MHz}, \mathrm{CDCl}_{3}\right)$
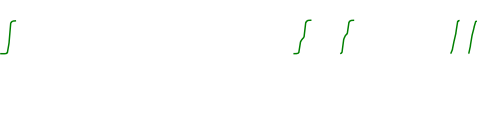


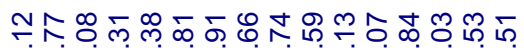

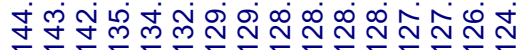

近

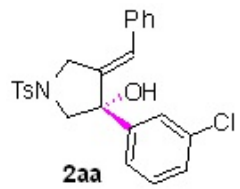

${ }^{13} \mathrm{C}$ NMR (126 MHz, $\mathrm{CDCl}_{3}$ )

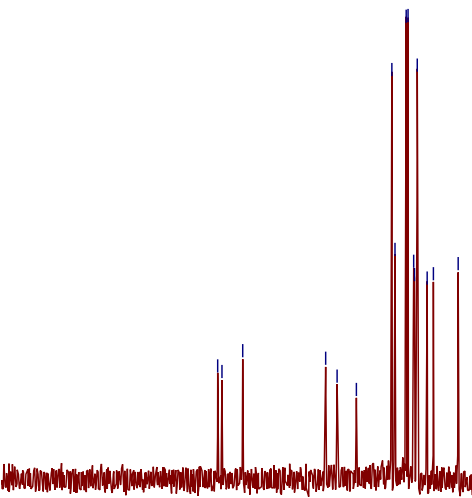

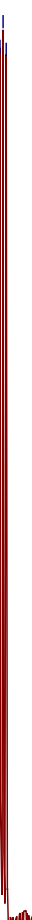

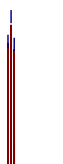



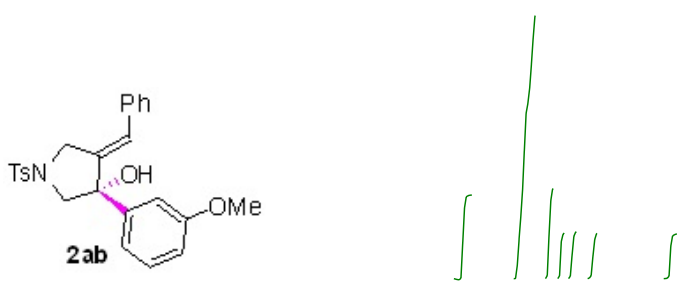

${ }^{1} \mathrm{H} \mathrm{NMR}\left(500 \mathrm{MHz}, \mathrm{CDCl}_{3}\right)$

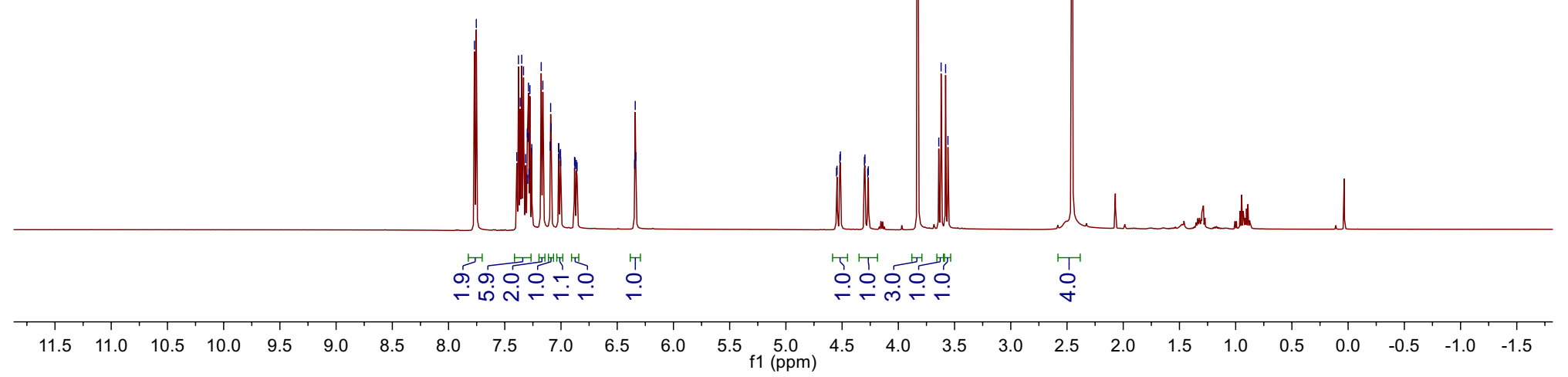




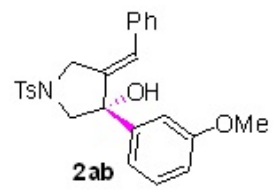

${ }^{13} \mathrm{C}$ NMR (126 MHz, $\left.\mathrm{CDCl}_{3}\right)$

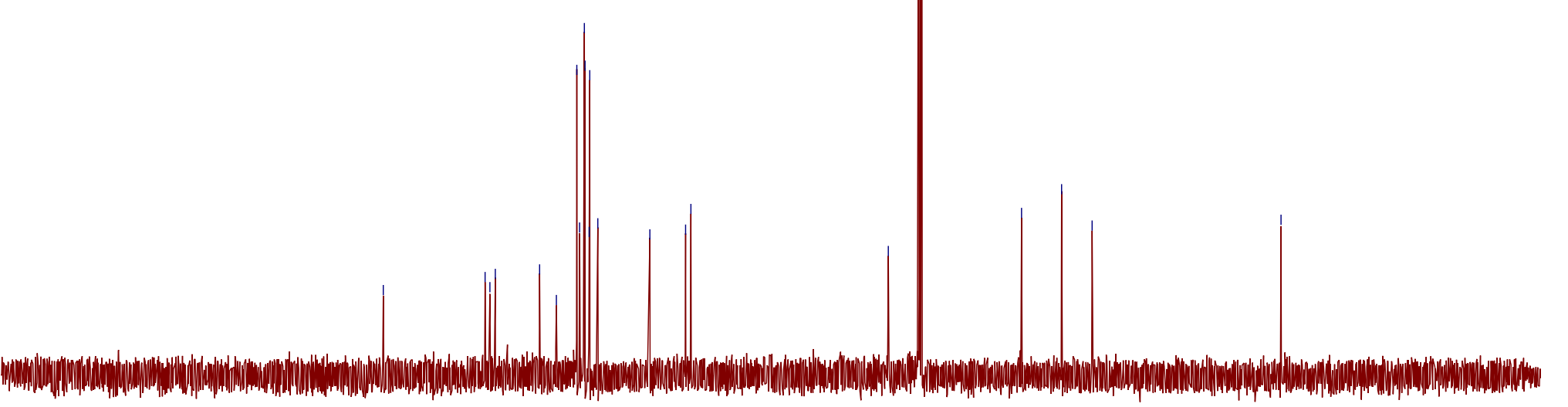

\begin{tabular}{|c|c|c|c|c|c|c|c|c|c|c|c|c|c|c|c|c|c|c|c|c|c|c|}
\hline 1 & $T$ & 1 & 1 & 1 & 1 & 1 & 1 & 1 & 1 & 1 & 1 & 1 & 1 & 1 & 1 & 1 & 1 & 1 & 1 & 1 & 1 & 1 \\
\hline 210 & 200 & 190 & 180 & 170 & 160 & 150 & 140 & 130 & 120 & 110 & $\begin{array}{c}100 \\
\mathrm{f} 1(\mathrm{ppm})\end{array}$ & 90 & 80 & 70 & 60 & 50 & 40 & 30 & 20 & 10 & 0 & -10 \\
\hline
\end{tabular}


品

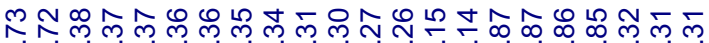

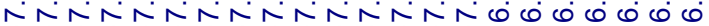
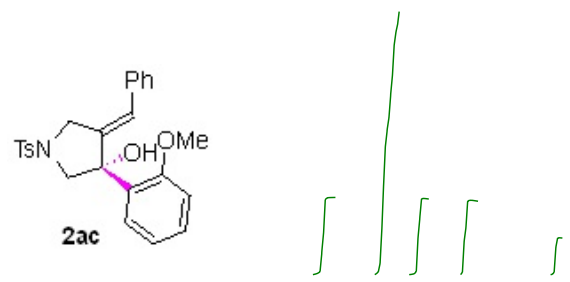

${ }^{1} \mathrm{H} \mathrm{NMR}\left(500 \mathrm{MHz}, \mathrm{CDCl}_{3}\right)$

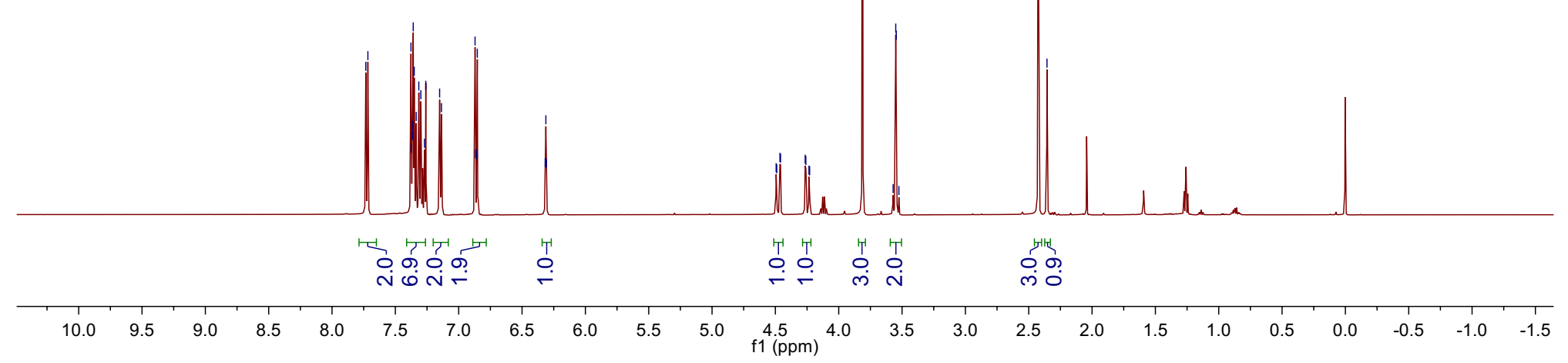




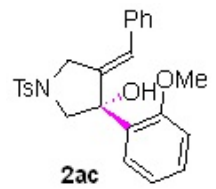

${ }^{13} \mathrm{C}$ NMR $\left(126 \mathrm{MHz}, \mathrm{CDCl}_{3}\right)$

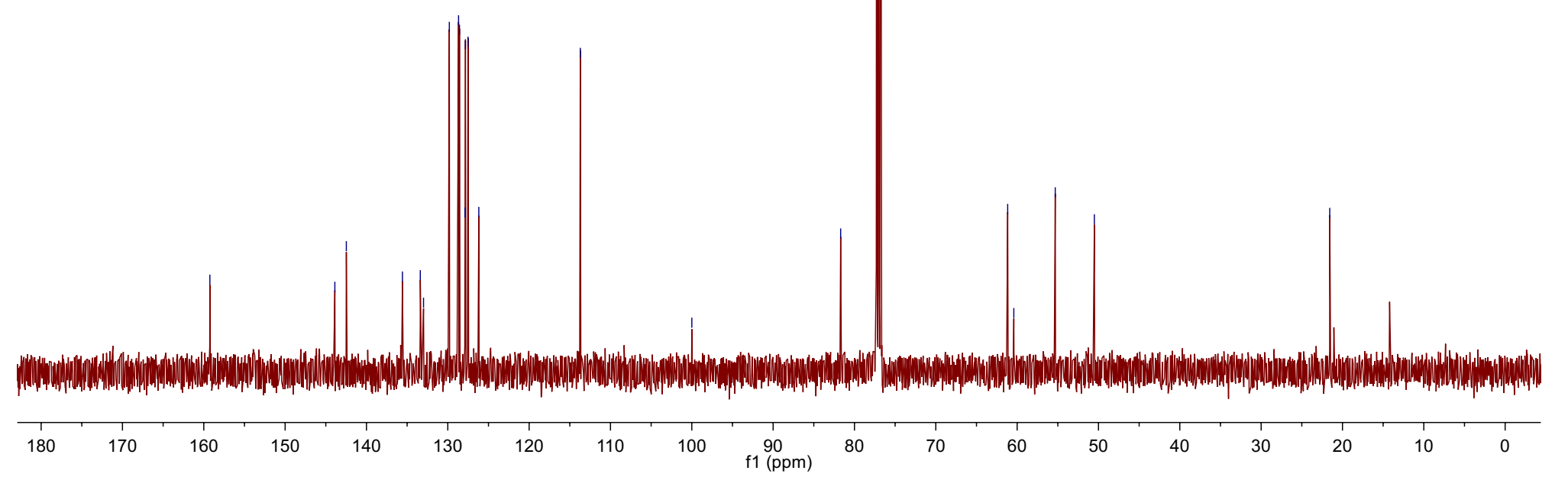



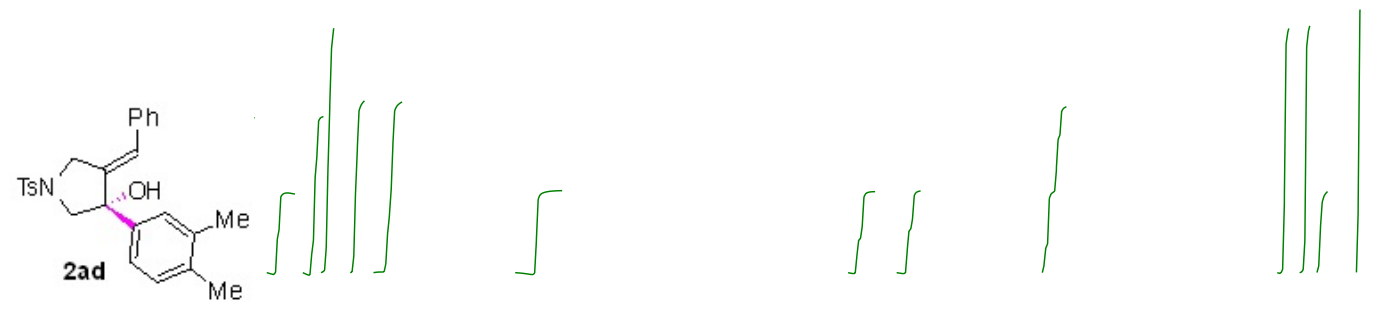

${ }^{1} \mathrm{H}$ NMR (500 MHz, $\mathrm{CDCl}_{3}$ )

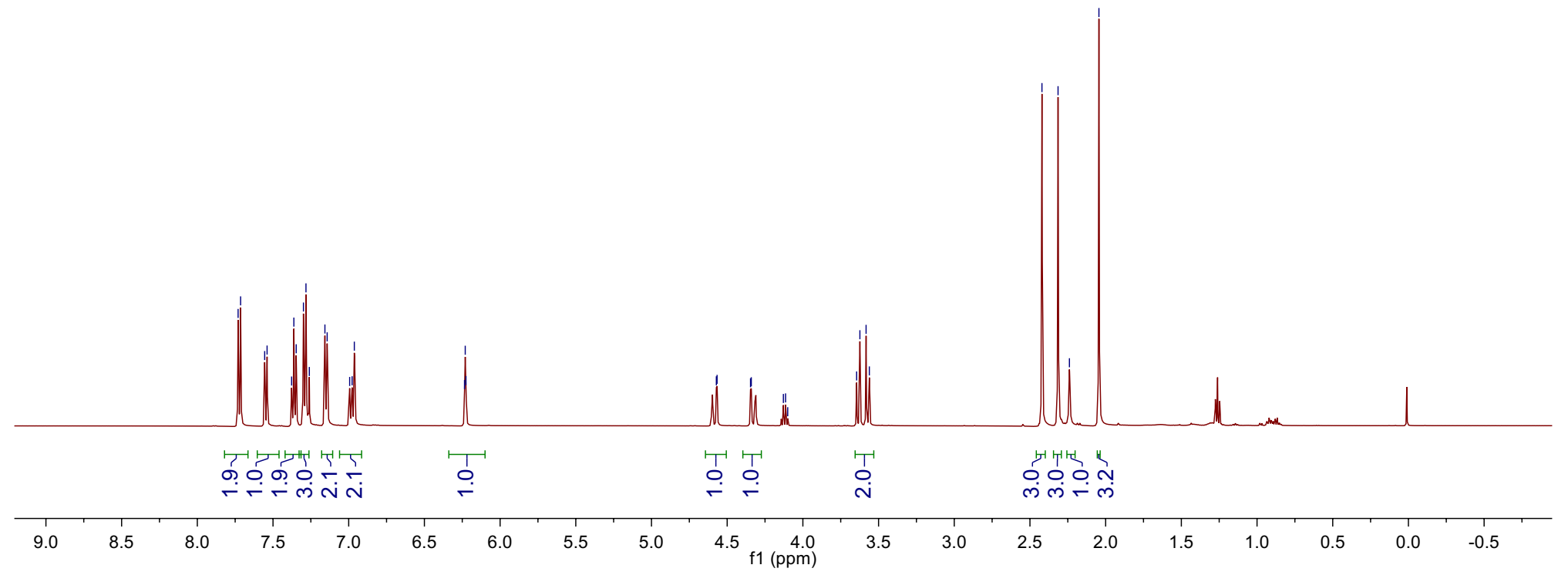


용ำ

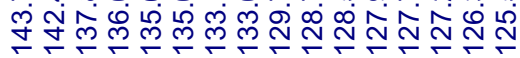

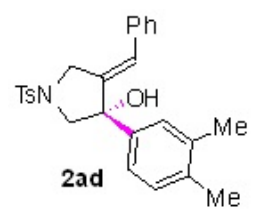

${ }^{13} \mathrm{C}$ NMR $\left(126 \mathrm{MHz}, \mathrm{CDCl}_{3}\right)$

(n) 

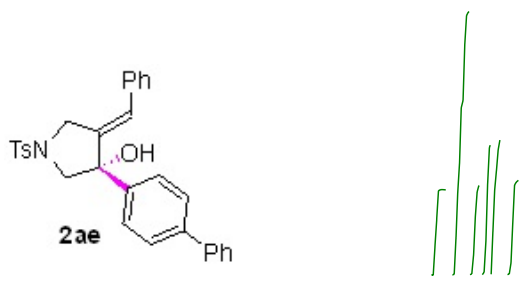

${ }^{1} \mathrm{H} \mathrm{NMR}\left(500 \mathrm{MHz}, \mathrm{CDCl}_{3}\right)$

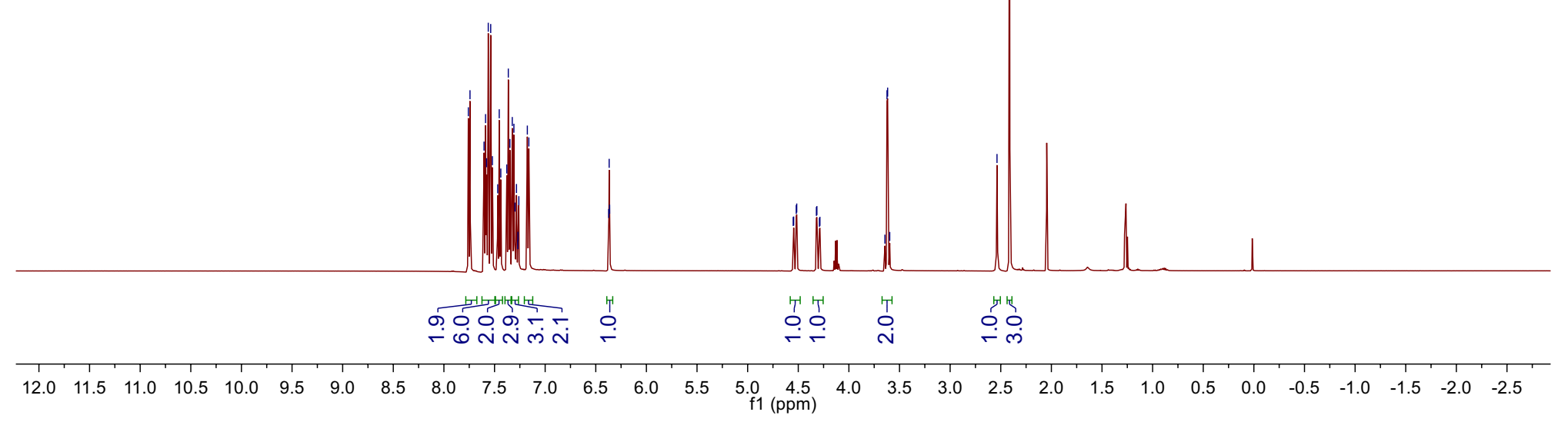




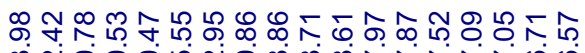

守守守守守宁

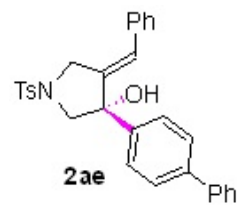

${ }^{13} \mathrm{C}$ NMR (126 MHz, $\mathrm{CDCl}_{3}$ ) 

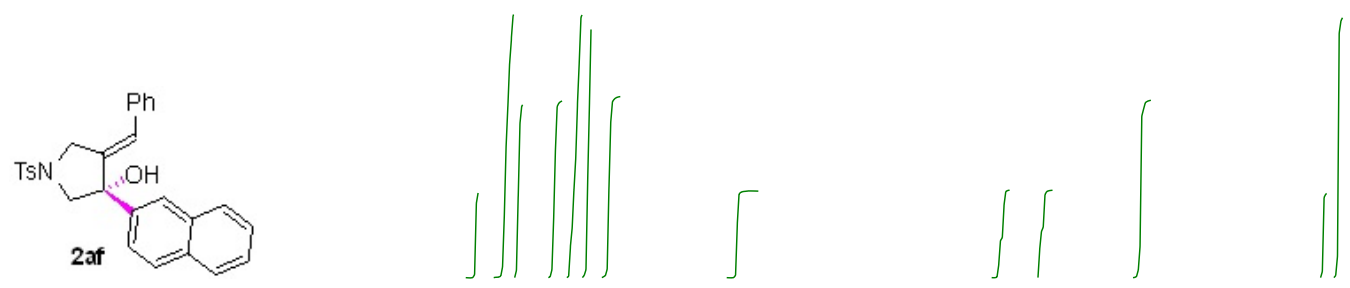

${ }^{1} \mathrm{H} \mathrm{NMR}\left(500 \mathrm{MHz}, \mathrm{CDCl}_{3}\right)$

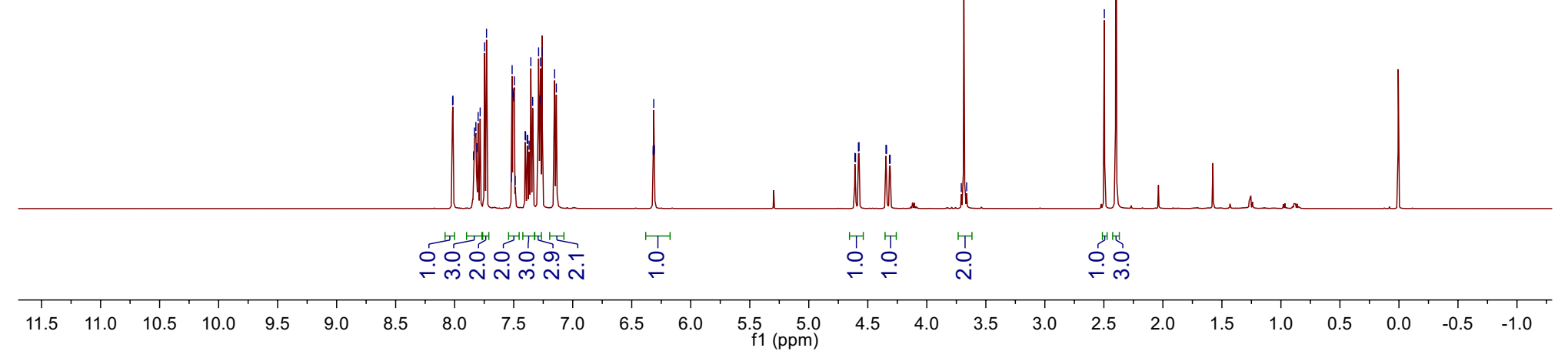




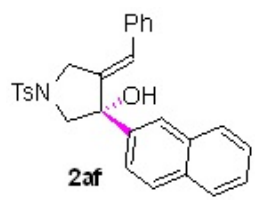

${ }^{13} \mathrm{C} \mathrm{NMR}\left(126 \mathrm{MHz}, \mathrm{CDCl}_{3}\right)$

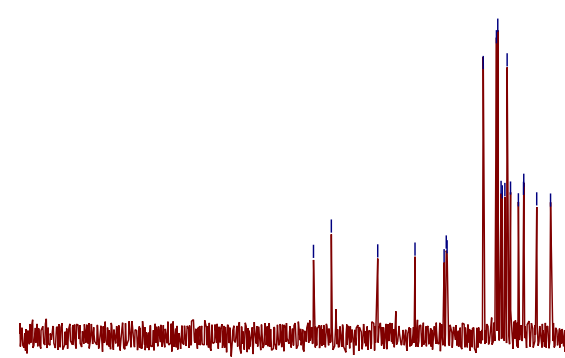

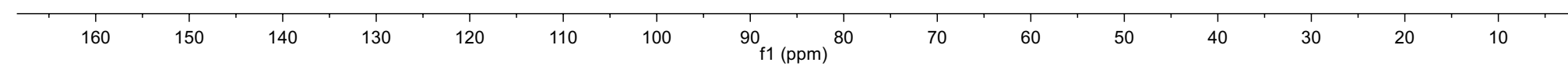


은

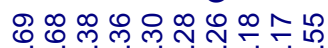

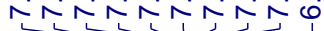

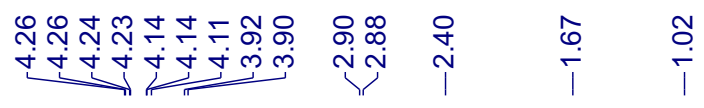

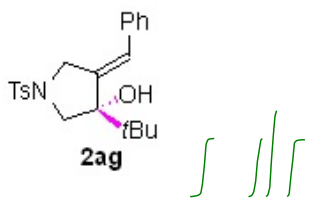

${ }^{1} \mathrm{H} \mathrm{NMR}\left(500 \mathrm{MHz}, \mathrm{CDCl}_{3}\right.$ )

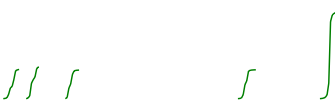

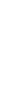

. 


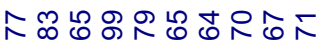

ชั่ำ

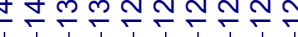

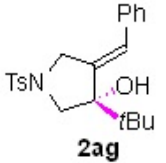

${ }^{13} \mathrm{C} \mathrm{NMR}\left(126 \mathrm{MHz}, \mathrm{CDCl}_{3}\right)$ $\infty$

นึ่

1 $\stackrel{\infty}{\leftarrow}$

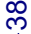

8 ก

กั

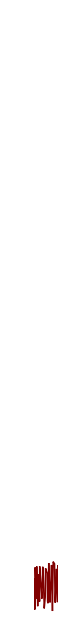




\section{鱼}

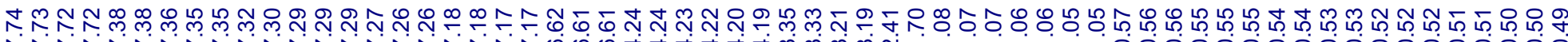

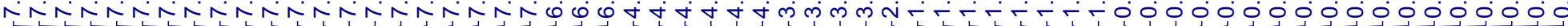
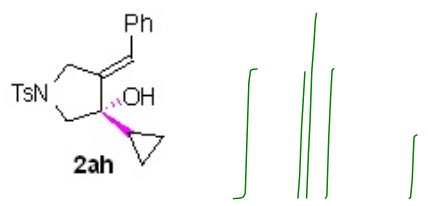

${ }^{1} \mathrm{H} \mathrm{NMR}\left(500 \mathrm{MHz}, \mathrm{CDCl}_{3}\right)$
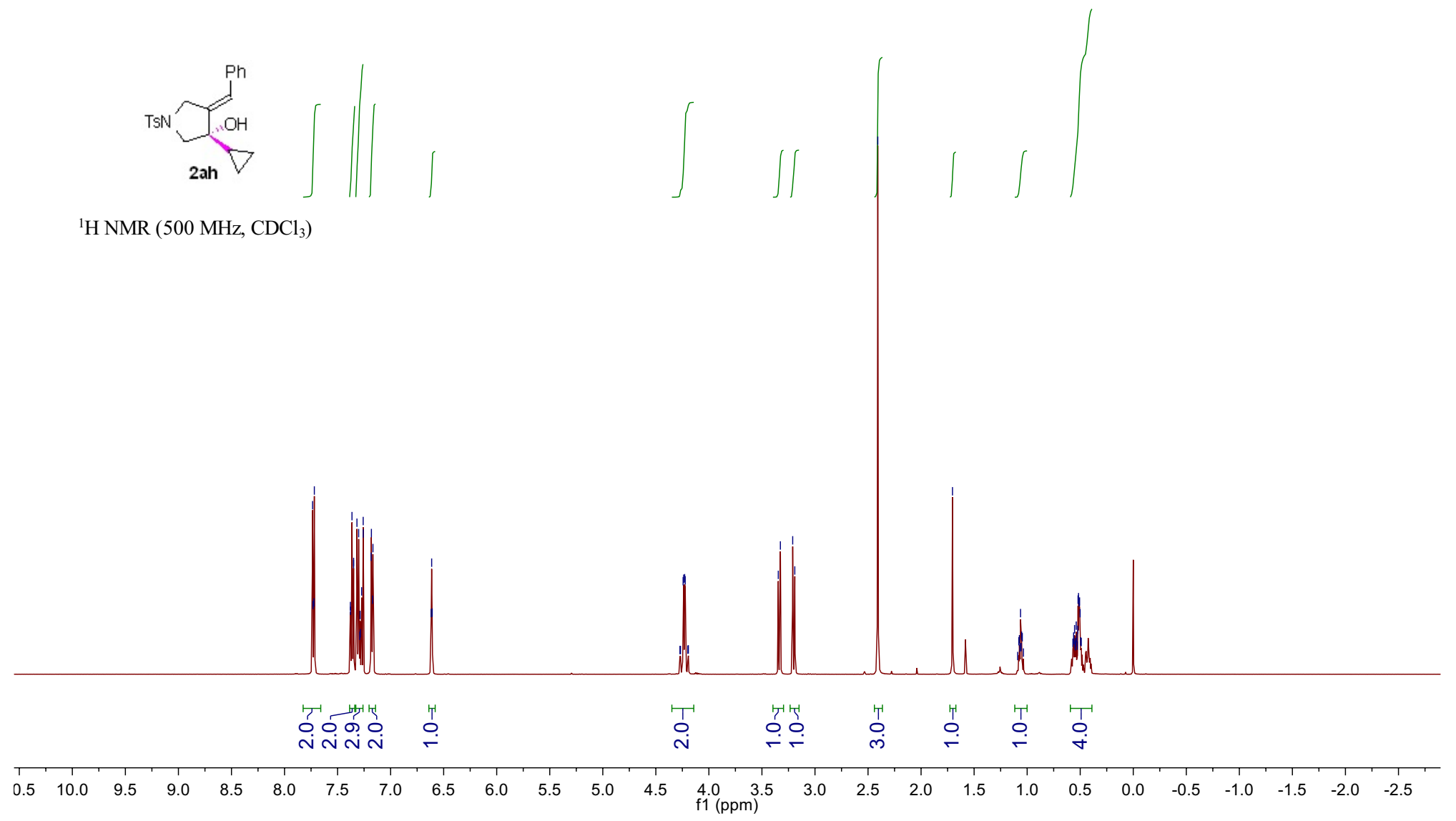
๓

守守

$\underbrace{-5+5}$

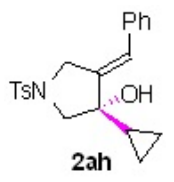

${ }^{13} \mathrm{C} \mathrm{NMR}\left(126 \mathrm{MHz}, \mathrm{CDCl}_{3}\right)$

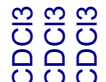

๑

余令

$\begin{array}{ll}10 & 0 \\ 0 & 0 \\ \infty & 0 \\ 0 & 0\end{array}$

苾

군

ํํำ

ij
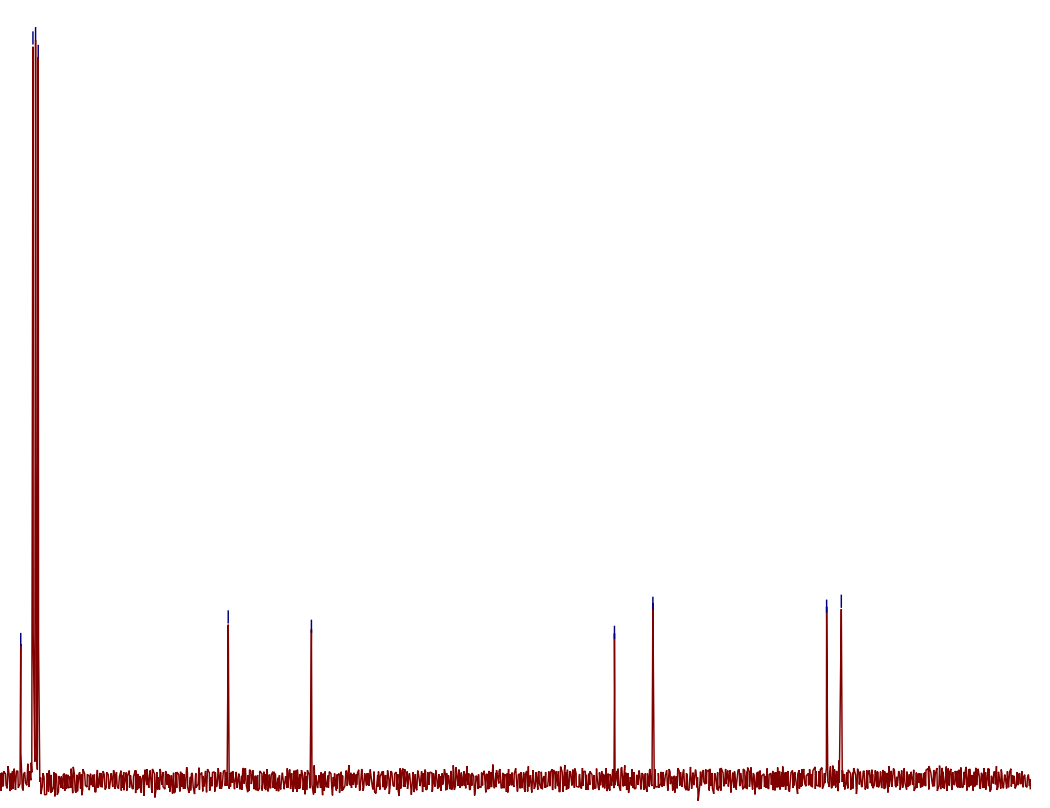
$\frac{m}{0}$

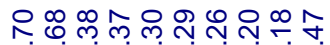
NNNNNNN

\section{ำ ำ}

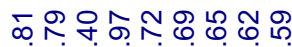

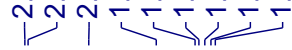

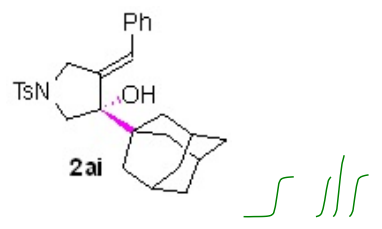

${ }^{1} \mathrm{H}$ NMR (500 MHz, $\mathrm{CDCl}_{3}$ )
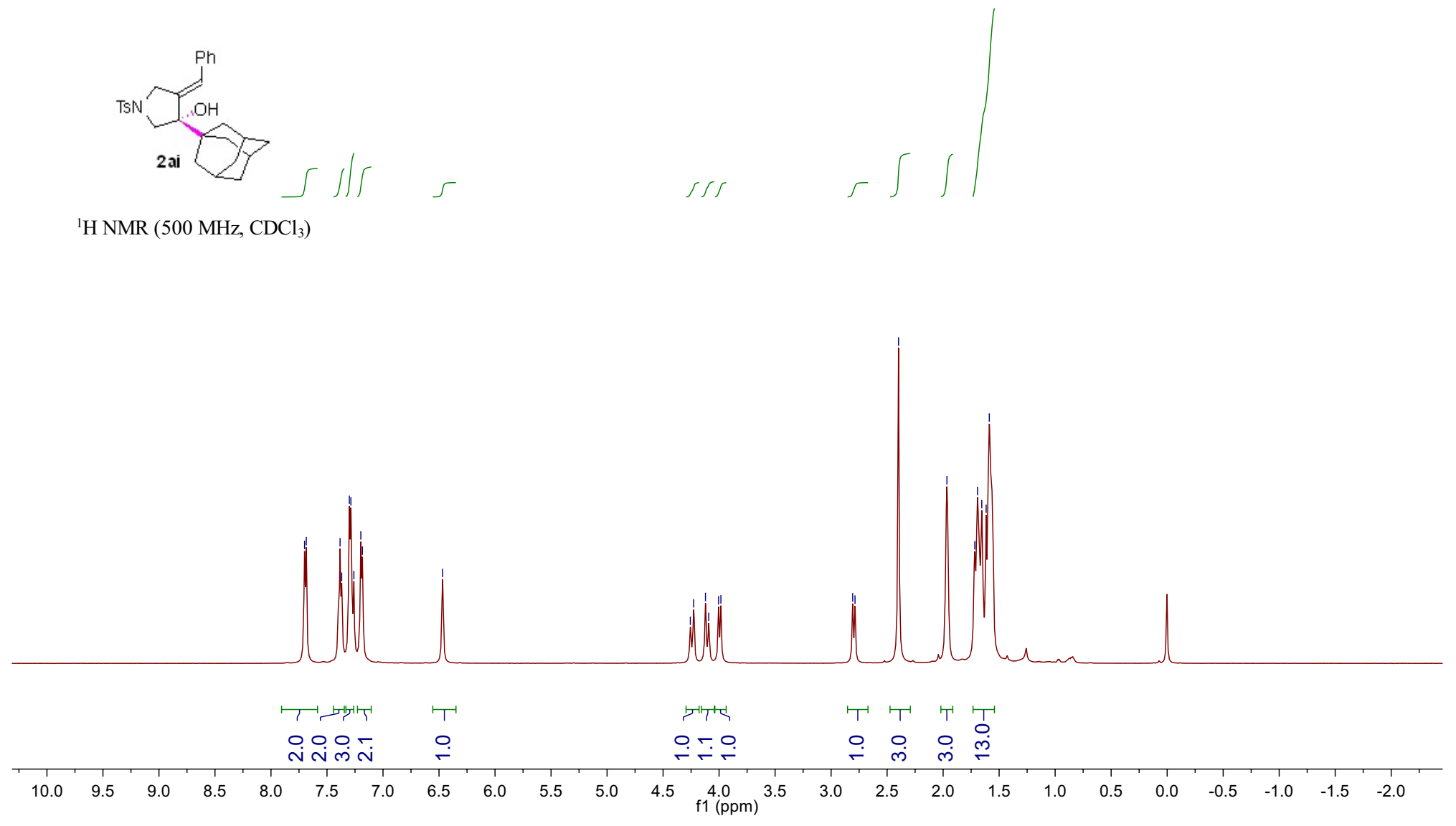


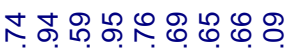

ঙ্ণু

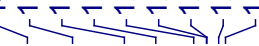

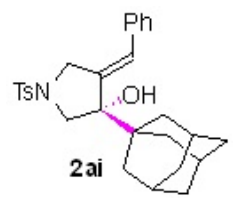

${ }^{13} \mathrm{C} \mathrm{NMR}\left(126 \mathrm{MHz}, \mathrm{CDCl}_{3}\right)$
高典焉

ป N

禅是

$$
\text { | }
$$

陨壬

ธำ

लंखि

กับ ำ

范

र।

$\stackrel{\sim}{i} \quad \stackrel{i}{i}$
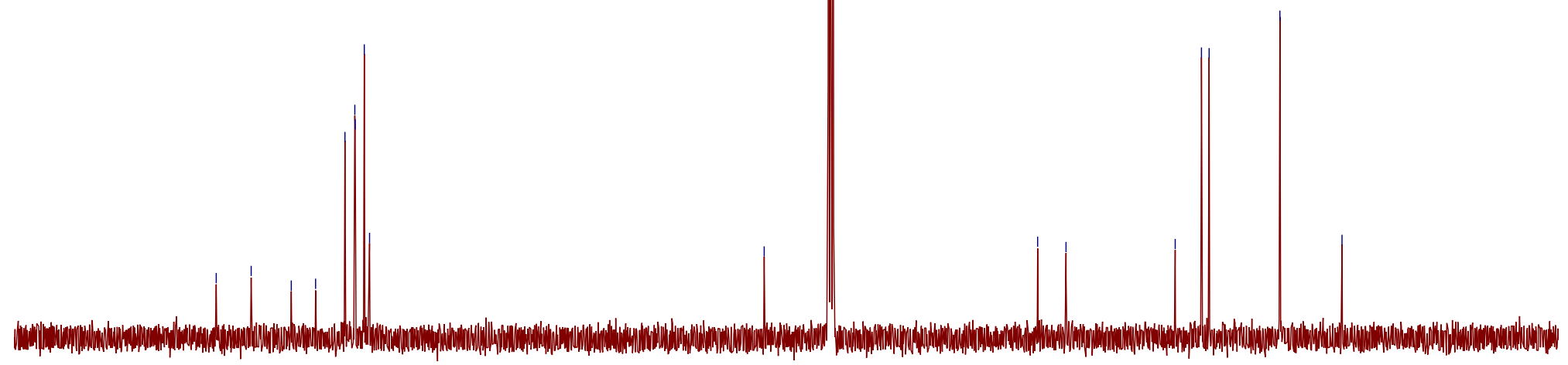

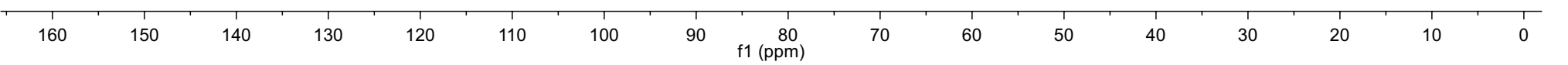




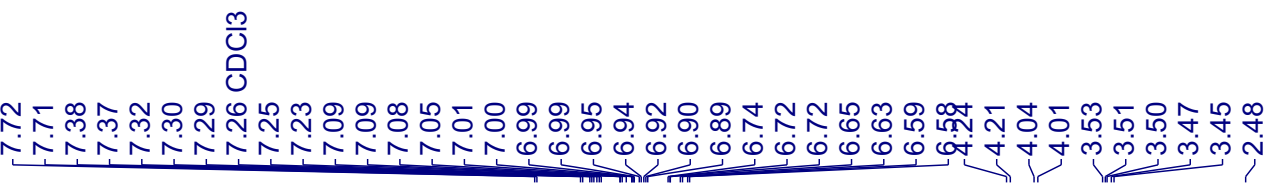<smiles>COc1ccc(C2(O)C[Al]CC2=C(c2ccccc2)c2ccccc2)cc1</smiles>
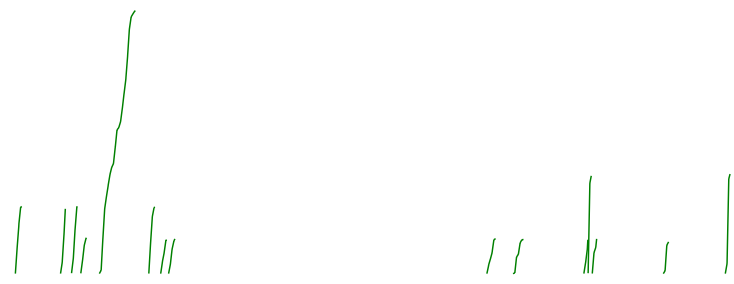

${ }^{1} \mathrm{H} \mathrm{NMR}\left(500 \mathrm{MHz}, \mathrm{CDCl}_{3}\right.$ )

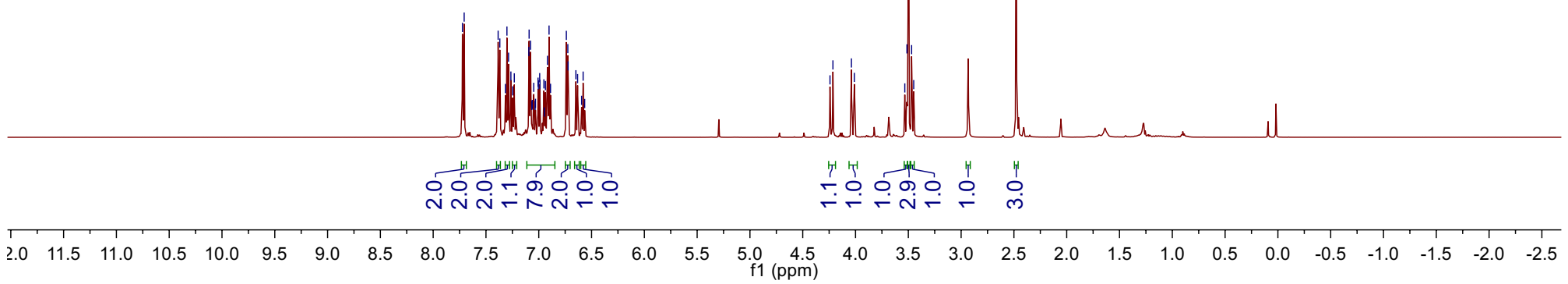




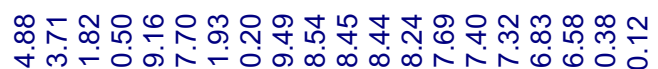

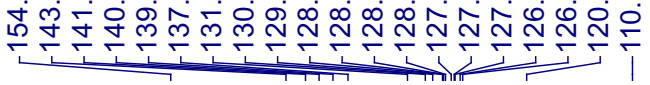<smiles></smiles>

${ }^{13} \mathrm{C}$ NMR (126 MHz, $\left.\mathrm{CDCl}_{3}\right)$

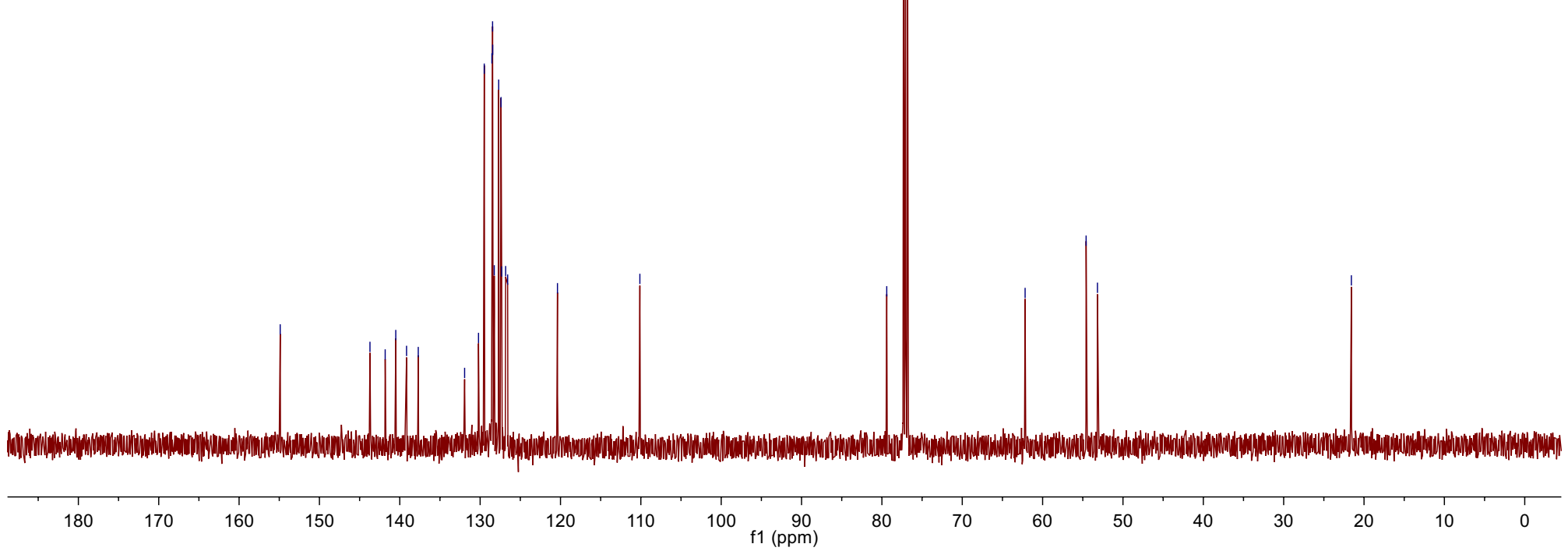

